University of Louisville

ThinkIR: The University of Louisville's Institutional Repository

Electronic Theses and Dissertations

$7-2008$

\title{
A possible role for SPLA2 in oligodendrocyte death and spinal cord injury.
}

William Lee Titsworth

University of Louisville

Follow this and additional works at: https://ir.library.louisville.edu/etd

\section{Recommended Citation}

Titsworth, William Lee, "A possible role for SPLA2 in oligodendrocyte death and spinal cord injury." (2008). Electronic Theses and Dissertations. Paper 1445.

https://doi.org/10.18297/etd/1445

This Doctoral Dissertation is brought to you for free and open access by ThinkIR: The University of Louisville's Institutional Repository. It has been accepted for inclusion in Electronic Theses and Dissertations by an authorized administrator of ThinkIR: The University of Louisville's Institutional Repository. This title appears here courtesy of the author, who has retained all other copyrights. For more information, please contact thinkir@louisville.edu. 


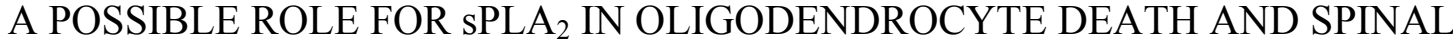 CORD INJURY
}

\author{
By \\ William Lee Titsworth \\ B.A. Samford University, 2001 \\ A Dissertation \\ Submitted to the Faculty of the \\ School of Medicine of the University of Louisville \\ in Partial Fulfillment of the Requirements \\ for the Degree of \\ Doctorate of Philosophy \\ Department of Anatomical Sciences and Neurobiology \\ University of Louisville \\ Louisville, Kentucky
}

July 10,2008 



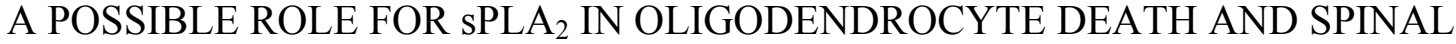 CORD INJURY
}

\author{
By \\ William Lee Titsworth \\ B.A., Samford University, 2001 \\ M.S., University of Louisville, 2007 \\ A dissertation Approved on
}

July 10,2008

By the following Dissertation Committee:

Xiao-Ming Xu, M.D., Ph.D.; Dissertation Director

Theo Hagg, Ph.D.

Charles H. Hubscher, Ph.D.

David S. Magnuson, Ph.D.

Meng S. Qiu, Ph.D. 


\section{DEDICATION}

This dissertation is dedicated to my wife Mary Beth Harrison Titsworth and sons Ashton

Lee and Luke William who with a hug and a smile brought me to places that my research could not. Also, to Glen Lee Titsworth and Linda Toohey Titsworth who support me without question or without regard to cost. I hope to one day show them the kindness that they have extended to me. 


\section{ACKNOWLEDGMENTS}

I would like to thank Xiao-Ming Xu, M.D. Ph.D., my advisor, for his tireless dedication to education and excellence. I will always call you, "Teacher."

I would like to thank Naikui Liu, M.D. Ph.D. If Dr. Xu was head of my project then you were its hands and its heart.

I would like to thank the Kentucky Spinal Cord Injury Research Center for their shared resources and watch care over me after Dr. Xu moved to Indianapolis. Particularly, I would like to thank Christine Nunn, Kim Fentress, and Aaron Pucket for animal care. I would like to thank Yi Ping Zhang, M.D. and Christopher B. Shield, M.D. for their surgical training and willingness to explore research design and troubleshoot. I would

like to thank Martha Bickford, Ph.D. for allowing access to her microscope and Neurolucida system and Scott R. Whittemore, Ph.D. for his understanding nature in allocating resources and his dedication to the M.D. / Ph.D. program.

This work was supported by NIH NINDS (XMX: NS36350, NS52290, NS50243; WLT: F31 S5657401), the Kentucky Spinal Cord and Head Injury Research Trust (\#4-16), the Daniel Heumann Fund for Spinal Cord Research, the James R. Petersdorf and Mari 
Hulman George Endowments. We also appreciate the use of the Center's Core facility supported by NIH COBRE RR15576.

Personally, I would like to thank my church, Hikes Point Christian Church, for their prayers and sanctuary. 


\begin{abstract}
A POSSIBLE ROLE FOR sPLA 2 IN OLIGODENDROCYTE DEATH AND SPINAL CORD INJURY

William Lee Titsworth
\end{abstract}

July 10, 2008

Spinal cord injury (SCI) can be divided into two distinct stages, an initial mechanical impact and a later "secondary injury" resulting from a cascade of cytokines triggering a spreading demyelination and apoptosis of neurons and glia within the spinal cord. It is believed that blockade of this "secondary injury" could improve functional and histological recovery following SCI. Here we propose that $\mathrm{sLA}_{2}$ might be one of the crucial mediators of the secondary injury. To test this possibility we first elucidated that the mRNA and protein of several isozymes of $\mathrm{sPLA}_{2}$ are present in the rodent spinal cord and that the group II enzymes are upregulated following SCI with a peak expression at 4 hours. Next, we showed that injuring differentiated cultures of oligodendrocyte precursor cells with $\mathrm{H}_{2} \mathrm{O}_{2}$ or TNF $\alpha$ and IL-1 $\beta$ induces sPLA $A_{2}$ expression and pharmacological inhibition with a sPLA 2 inhibitor, S3319, creates partial reversal of this injury. We further showed that a nanogram injection of $\mathrm{SPLA}_{2}$ into the naïve dorsolateral funiculus of the cervical spinal cord is sufficient to produce demyelination, axonopathy, and glial death as well as a dose dependent loss of function as measured by pellet retrieval. Finally we showed that inhibition of sPLA 2 by either i.p. injections of S3319 or a frame shift 
mutation in the sPLA $\mathrm{A}_{2}$-IIA gene creates functional improvements in overground locomotion and bladder function. The functional recovery correlates well with increased white matter sparing and oligodendrocyte numbers within in the spinal cord, increased axon numbers at the lesion epicenter, and decreased inflammation and lesion cavity volume. These findings suggest that $\mathrm{SPLA}_{2}$ may play an important role in secondary SCI and that its blockade could facilitate recovery following SCI. 
TABLE OF CONTENTS

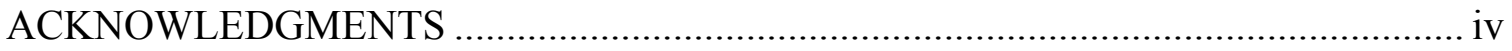

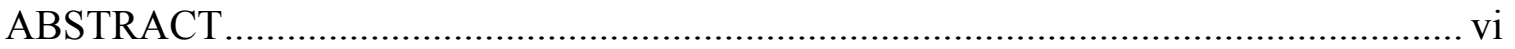

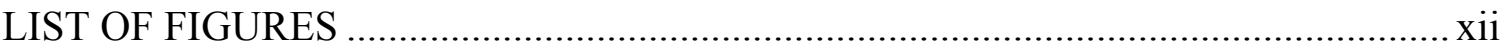

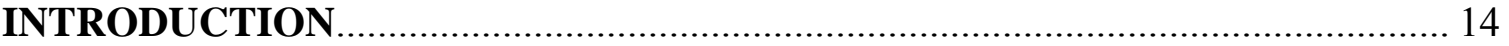

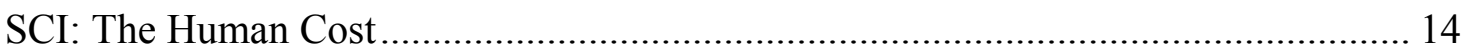

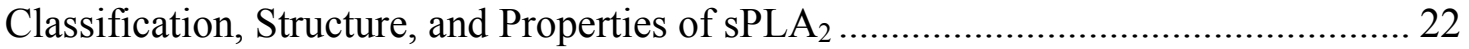

Suggested Mechanisms underlying sPLA 2 -Induced CNS Injury ……....................... 32

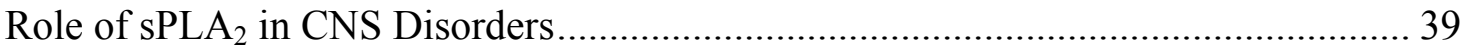

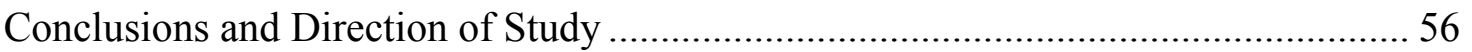

EXPRESSION OF SPLA $A_{2}$ FOLLOWING SPINAL CORD INJURY …………...... 57

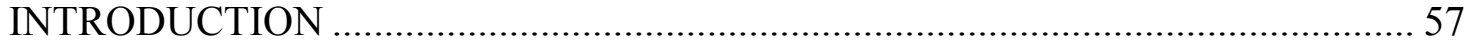

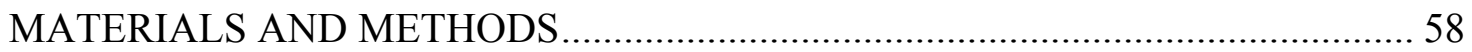

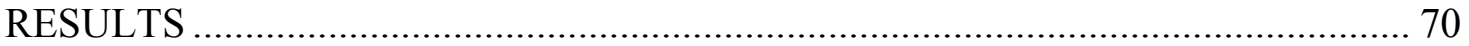

${ }_{s P L A}$ mRNAs are differentially expressed following SCI..................................... 70

${ } \mathrm{PLA}_{2}$ proteins are differentially expressed following SCI..................................... 74

Spatiotemporal distribution of sPLA $\mathrm{P}_{2}$ isozymes following $\mathrm{SCI}$.............................. 77

Cellular localization of sPLA 2 isozymes following SCI......................................... 81

${ }_{\text {PLA }}$-IIA increases in membrane fraction following SCI. .................................... 84 
sPLA $\mathrm{A}_{2}$ attenuates $\mathrm{H}_{2} \mathrm{O}_{2}$ induced oligodendrocyte death in vitro. 92

${ }_{\mathrm{sPLA}}$ mediates IL-1 $\beta$ and TNF $\alpha$ induced oligodendrocyte injury in vitro. 98

DISCUSSION 101

\section{SPLA $_{2}$ INJECTIONS INTO THE NAÏVE SPINAL TISSUE RESULT IN A DOSE}

DEPENDENT LOSS OF FUNCTION AND TISSUE DAMAGE 107

INTRODUCTION 107

MATERAILS AND METHODS ..................................................................... 108

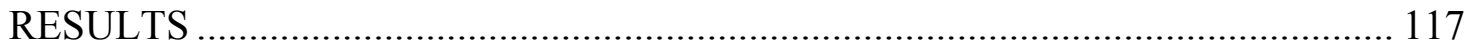

sPLA $_{2}$-III Creates a Consistent Demyelination and Remyelination of the Spinal Cord

DISCUSSION 136

SPLA $A_{2}$ INHIBITION PRIOR TO SPINAL CORD INJURY RESULTS IN

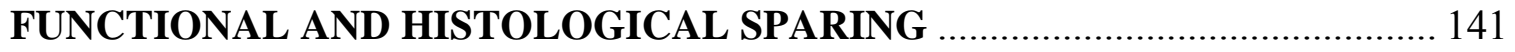

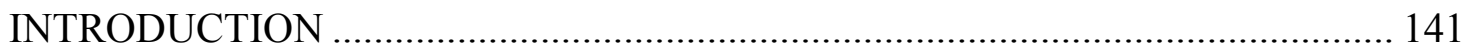

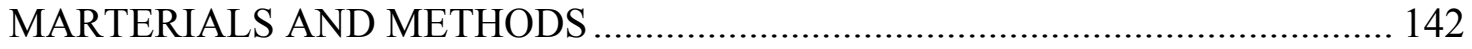

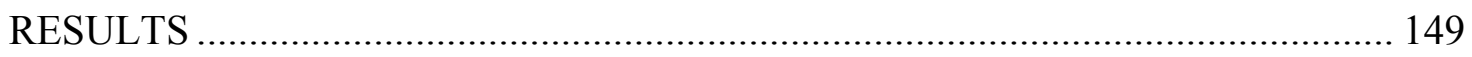

Inhibition of $\mathrm{sPLA}_{2}$ Increases Behavioral Recovery Following SCI.................... 149

Inhibition of $\mathrm{sPLA}_{2}$ Increases Histological Recovery Following SCI................... 154

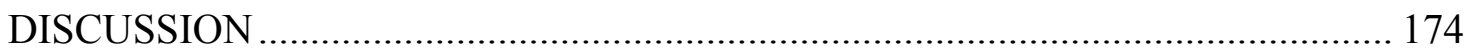

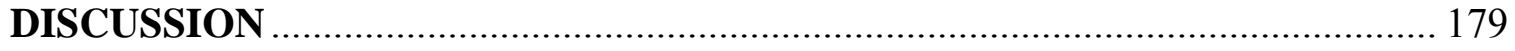

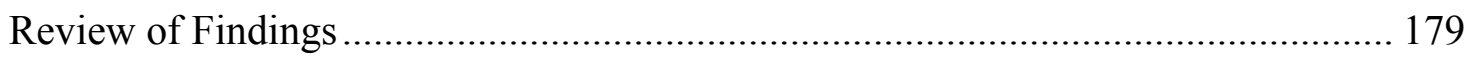

Limitations of Current Findings..................................................................... 182

Application of Findings to Future sPLA 2 Research:........................................ 187 


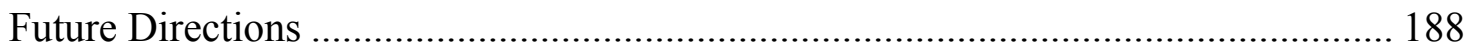

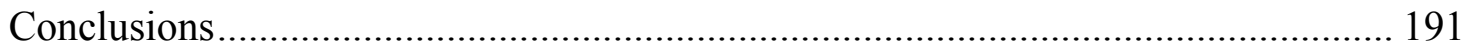

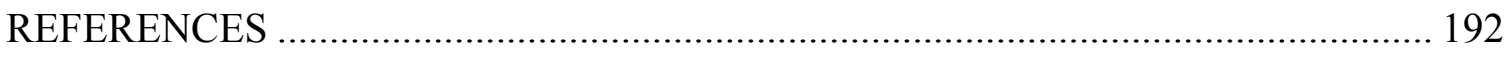

APPENDIX

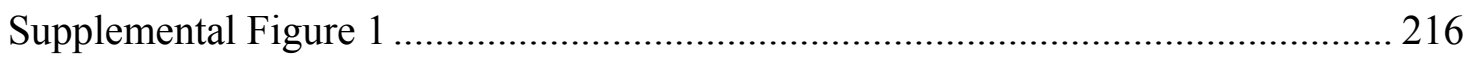

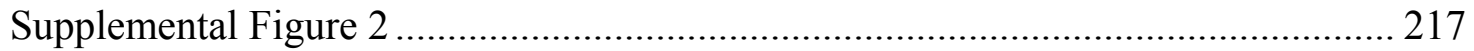

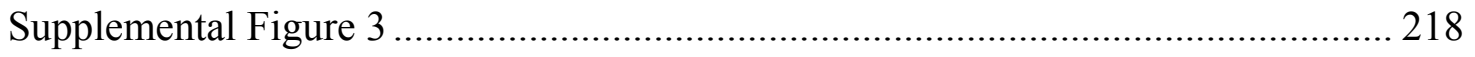

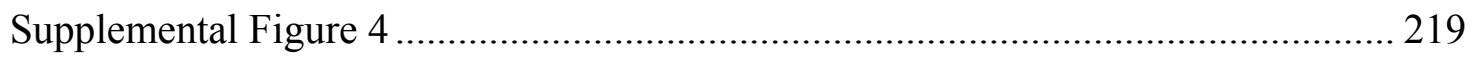

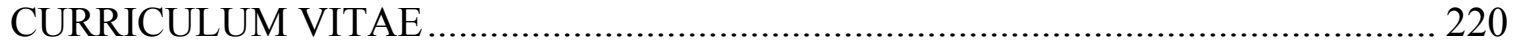




\section{LIST OF TABLES}

TABLE

PAGE

1. Animal Usage and Experimental Groups (Study \#1) ...........................59

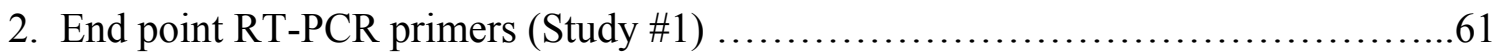

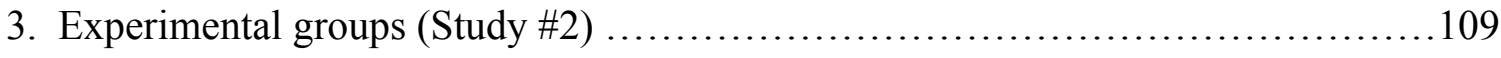

4. Demyelination Parameters (Study \#2) ..................................... 118

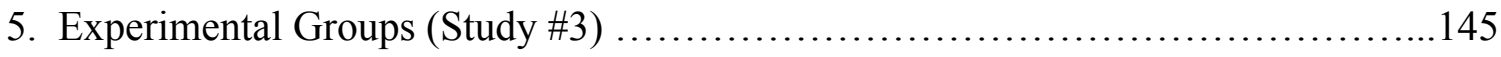

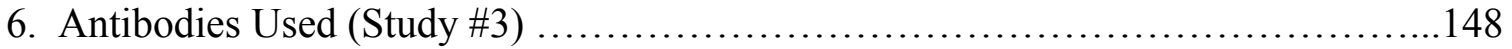

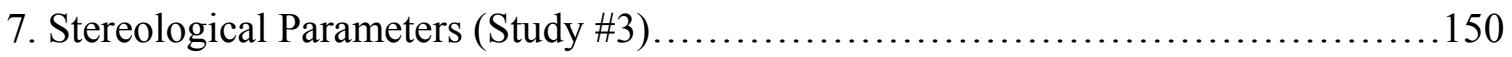




\section{LIST OF FIGURES}

FIGURE

PAGE

Figure 1. Classification of the Mammalian PLA $\mathrm{PL}_{2}$ Isoforms. ......................................... 17

Figure 2. Intracellular Handling and sPLA 2 Activity .................................................... 24

Figure 3. Overview of sPLA $A_{2}$ 's Role in Spinal Cord Injury......................................... 33

Figure 4. sPLA 2 Activity Within Spinal Cord Injury. ................................................... 43

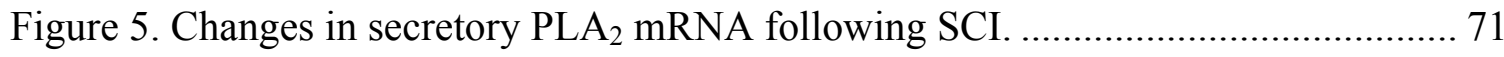

Figure 6. Temporal changes in $\mathrm{PPLA}_{2}$ protein expression following SCI....................... 75

Figure 7. Immunohistochemical Changes in $\mathrm{SPLA}_{2}$-IB, IIA, IIE, and X Expression

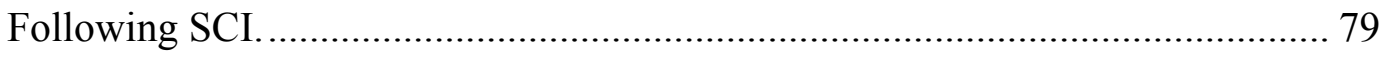

Figure 8. Co-localization of sPLA $\mathrm{A}_{2}$ Isoforms in Oligodendrocytes at $4 \mathrm{hr}$ Post-SCI. ...... 82

Figure 9. sPLA $\mathrm{PL}_{2}$-IIA Expression Increases in Oligodendrocytes Following SCI and

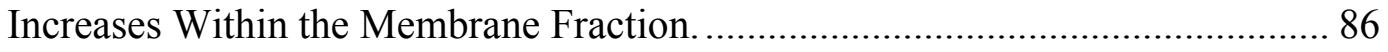

Figure 10. Oligodendrocytes show sensitivity to $\mathrm{SPLA}_{2}$-IIA triggered cell death relative

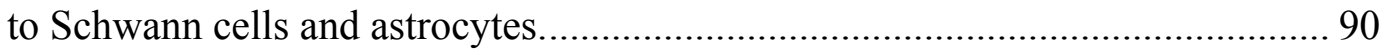

Figure 11. $\mathrm{H}_{2} \mathrm{O}_{2}$ Injury Induces sPLA $\mathrm{A}_{2}$-IIA Expression in Cultured Oligodendrocytes... 93

Figure 12. $\mathrm{sPA}_{2}$-IIA Enzymatic Inhibition Partially Reversed $\mathrm{H}_{2} \mathrm{O}_{2}$ Induced

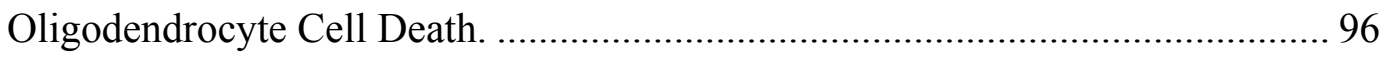

Figure 13. Blockade of $\mathrm{sPLA}_{2}$ in Oligodendrocyte Cultures Prevents Morphological

Changes and Death Associated with IL-1 $\beta$ and TNF $\alpha$ Injury ............................... 99 
Figure 14. Demyelination Occurring After Unilateral Cervical (C2/3) Spinal Cord

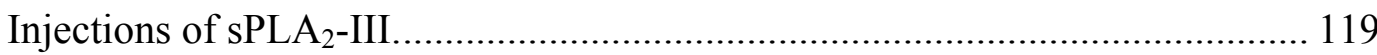

Figure 15. Histological Results Occurring 4 Weeks After Unilateral Cervical (C2/3)

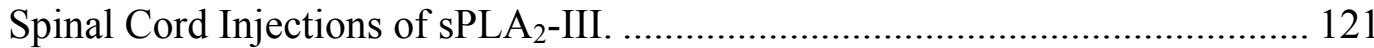

Figure 16. Immunofluorescent labeling and quantification of axons during 4 weeks after unilateral cervical $(\mathrm{C} 2 / 3)$ spinal cord injections of sPLA $_{2}$-III. 124

Figure 17. Immunofluorescent double labeling shows a progressive remyelination of spared axons by Schwann cells over a 4 week period. 126

Figure 18. Video captured images of pellet retrieval test performance prior to injury (AD) and 1 week following $3 \mathrm{ng}$ injection of $\mathrm{sPLA}_{2}-\mathrm{III}(\mathrm{E}-\mathrm{H})$.

Figure 19. Behavioral results occurring after unilateral cervical $(\mathrm{C} 2 / 3)$ spinal cord injections of $\mathrm{sPLA}_{2}$-III

Figure 20. $\mathrm{sPLA}_{2}$ Inhibition Increases Functional Recovery Following Severe Spinal Cord Injury 152

Figure 21. sPLA $\mathrm{A}_{2}$ Inhibition Increases Myelin Sparing ........................................... 156

Figure 22. sPLA $_{2}$-IIA Immunoreactivity Co-localizes with Demyelinated Tissue ....... 158

Figure 23. sPLA $\mathrm{A}_{2}$ Inhibition Results in Decreased Inflammation Following SCI......... 160

Figure 24. sPLA $_{2}$ Effect on Glial Lesion and Axons Within the Epicenter ................. 164

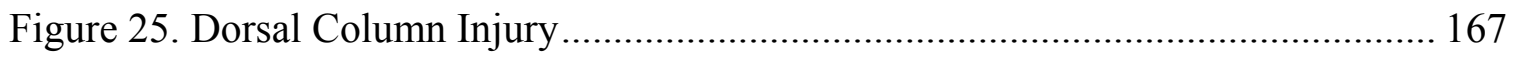

Figure 26. sPLA $_{2}$ Inhibition Increases Oligodendrocytes Numbers Following SCI. ... 170

Figure 27. Comparison of Histological Outcomes to Behavioral Function................... 172 


\section{CHAPTER I}

\section{INTRODUCTION}

\section{SCI: The Human Cost}

Modern advances in treatment for spinal cord injury (SCI) have resulted in the saving of lives but with only limited gains in function. This in turn has contributed to an increasing societal cost. The National Spinal Cord Injury Statistical Center at the University of Alabama in Birmingham estimated in the 1970's that there were 12,000 new cases of spinal cord injury (SCI) each year. Data from 2007 indicates that between 230,000 and 300,000 people are currently living with a SCI. In 2005, the average length of stay in a hospital was 15 days following a SCI with an additional 36 days in a rehab unit. The average yearly health care and living expenses for a 25 year old patient with high tetraplegia is $\$ 775,567$ with an estimated direct lifetime cost of $\$ 3,059,184$ (NSCISC, 2008).

Many of the current theories involving spinal cord injury (SCI) divide the process into two stages, an initial mechanical damage to the cord tissue followed by a cascade of "secondary injury." The secondary injury is thought to be an immune response to damaged tissue that in turn leads to widespread neuronal and glial cell death as well as demyelination (McTigue et al., 2001, Cao et al., 2005, Totoiu and Keirstead, 2005). Notably, oligodendrocytes are particularly sensitive to apoptosis during secondary injury, which results in a loss of myelin around surviving axons peripheral to the lesion epicenter 
(Blight, 1985, Crowe et al., 1997, Totoiu and Keirstead, 2005). By seven days post injury, $93 \%$ of the oligodendrocytes at the impact site are lost (McTigue et al., 2001). Since only accident prevention can attenuate the initial mechanical damage from SCI, it is hypothesized that therapeutic manipulation of the inflammatory "secondary injury" could result in increased oligodendrocyte survival after neurotrauma and that this represents a viable approach to restore functional conduction of intact but demyelinated axons (Jones et al., 2005b).

Therefore, much of the current work in SCI has revolved around muting the inflammatory response. Examples of this work include blockade of the TNF $\alpha$ receptor (Demjen et al., 2004), injection of the anti-inflammatory antibiotic minocycline (Wells et al., 2003), the systemic depletion of macrophages (Popovich et al., 1999) and neutrophils (Taoka et al., 1997), use of immunosuppressant pharmaceuticals such as cyclosporine A (Rabchevsky et al., 2001). However, the complex role of inflammation suggests that other inflammatory mediators could be present following SCI and their blockade could result in therapeutic interventions, possibly even a multi-modal approach. Therefore we have suggested that phospholipases $\mathrm{A}_{2}$, an immerging class of proinflammatory molecules, might play a role in secondary SCI.

Phospholipases $\mathrm{A}_{2}$ (EC 3.1.1.4) are enzymes that catalyze the hydrolysis of the sn-2 position of membrane glycerophospholipids, leading to the production of free fatty acids and lysophospholipids. These enzymes are of particular interest since free fatty acids can be converted to bioactive eicosanoids via the cyclooxygenase pathway leading to increased inflammation. Additionally, the other reaction product, lysophospholipids, such as lysophosphatidic acid and lysophosphatidylcholine (LPC), are also bioactive 
(Lauber et al., 2003) and can be converted into platelet-activating factor (PAF). Since lipids compose a disproportionately large amount of the CNS and phospholipids constitute $44 \%$ of myelin (Morell, 1984), understanding the role of phospholipases in CNS disorders becomes a major priority.

To date, more than 27 mammalian isoforms of $\mathrm{PLA}_{2}$ have been found which can be classified into four major categories: secretory $\mathrm{PLA}_{2}\left(\mathrm{sPA}_{2}\right)$, cytosolic $\mathrm{PLA}_{2}$ $\left(\mathrm{cPLA}_{2}\right), \mathrm{Ca}^{2+}$-independent $\mathrm{PLA}_{2}\left(\mathrm{iPLA}_{2}\right)$, and PAF acetylhydrolases (PAF-AH) (Murakami et al., 1997, Murakami and Kudo, 2001, Kudo and Murakami, 2002, Schaloske and Dennis, 2006). See Fig. 1. 
Figure 1. Classification of the Mammalian PLA 2 Isoforms.

The top panel shows a branching diagram indicating the relative subdivisions of the PLA 2 subfamily and their years of discovery. The mammalian PLA family of enzymes is grossly divided into the $\mathrm{SPLA}_{2}, \mathrm{cPLA}_{2}, \mathrm{iPLA}_{2}$, and PAF-AH. The sPLA 2 subfamily is further divided into groups IB, group II and V, group X, and group III and XII based on structural and functional differences presented in the table below. HSPG: heparin sulfate proteoglycans 


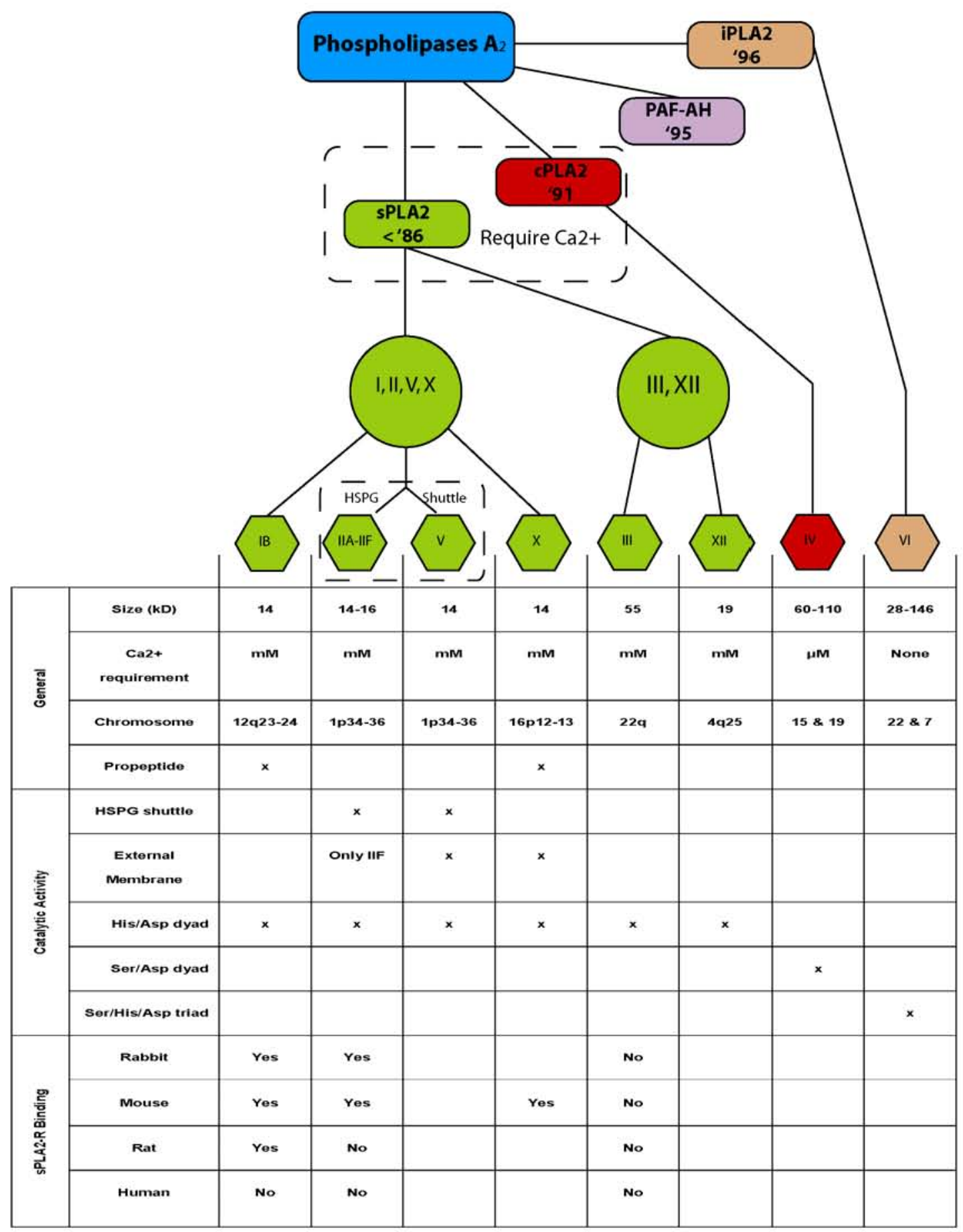


Our previous work demonstrated that both total $\mathrm{PLA}_{2}$ activity and cPLA $\mathrm{A}_{2} \alpha$ ( $\left.\mathrm{PLA}_{2}-\mathrm{IV} \alpha\right)$ protein expression increased following SCI (Liu et al., 2006). However, total $\mathrm{PLA}_{2}$ activity peaked at $4 \mathrm{hr}$ while $\mathrm{cPLA}_{2} \alpha\left(\mathrm{PLA}_{2}\right.$-IV $\left.\alpha\right)$ protein did not significantly increase until 7 days post injury. This paradox suggests that another isoform of $\mathrm{PLA}_{2}$ might be responsible for the increase in total phospholipases activity after SCI. For this reason we chose to investigate the role that the various $\mathrm{SPLA}_{2}$ isoforms might play following SCI.

The 11 mammalian isozymes in the sPLA 2 subfamily all have a low molecular mass of about $14-18 \mathrm{kD}$, require the presence of submillimolar to millimolar concentrations of $\mathrm{Ca}^{2+}$ for effective hydrolysis of a substrate phospholipid, and lack fatty acid selectivity (Dennis, 1994, Kudo and Murakami, 2002, Schaloske and Dennis, 2006). The functions of $\mathrm{sPLA}_{2} \mathrm{~s}$ are far reaching, including digestion, exocytosis (Matsuzawa et al., 1996), and anticoagulation (Kini and Evans, 1987). However, sPLA ${ }_{2} \mathrm{~s}$ most prominent role is in inflammation following pathological conditions such as infections (Laine et al., 1996, Weinrauch et al., 1996, Laine et al., 2000), ischemia (Lauritzen et al., 1994), atherosclerosis (Ivandic et al., 1999, Leitinger et al., 1999, Tietge et al., 2000), and cancer (MacPhee et al., 1995, Cormier et al., 1997, Morioka et al., 2000a, Takaku et al., 2000).

\section{Role in General Inflammation}

$\mathrm{sPA}_{2}$ has had an established role in inflammation and inflammatory diseases for some time (Nevalainen et al., 2000). The blockade of $\mathrm{PLA}_{2}$ holds a particular interest for pharmacologists since inhibition of $\mathrm{sPLA}_{2}$ would in theory prevent the formation of inflammatory eicosanoids prior to the cyclooxygenase (COX; EC 1.14.99.1) reaction. In 
fact, $\mathrm{PLA}_{2}$ is the rate limiting precursor in arachidonic acid (AA) production (Schaefers et al., 1996). Therefore its blockade should eliminate the need for COX-1 versus COX-2 specificity in anti-inflammatory therapeutics. This theory has spurred the development of a large number of $\mathrm{sPLA}_{2}$ inhibitors that unfortunately, have not produced the desired clinical efficacy to date (Reid, 2005).

$\mathrm{SPA}_{2} \mathrm{~s}$ have been linked to many inflammatory diseases. $\mathrm{SPLA}_{2}$ activity is elevated in several body fluids of patients with acute pancreatitis (Makela et al., 1990). Synovial fluid from arthritic joints of rheumatic patients contains sPLA 2 -IIA (Kramer et

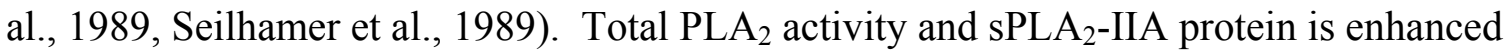
in bronchoalveolar lavage fluids from patients with adult respiratory distress syndrome (Kim et al., 1995). Increased levels of sPLA 2 -IIA were seen in the skin of patients with psoriasis (Andersen et al., 1994). Increased group II, PLA $_{2}$ expression was found in colonic mucosa of patients with Crohn's Disease and ulcerative colitis (Minami et al., 1994) and experimental models of ischemic bowel disease in rodents (Otamiri et al., 1988, Fabia et al., 1993). Additionally, serum levels of sPLA 2 , particularly group IIA, increase in patients with sepsis (Green et al., 1991, Keuter et al., 1995) and injuries (Uhl et al., 1990, Nevalainen et al., 2000), and following many types of surgeries such as cardiac surgery (Gronroos et al., 1995), aortobifemoral reconstruction (Gronroos et al.,

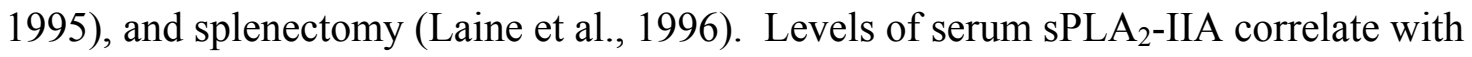
C-reactive protein in several disease states, supporting the notion that $\mathrm{PLA}_{2}$-IIA is an acute phase protein (Crowl et al., 1991). Some suggest that elevations in serum levels of ${ }_{s P L A}$-IB are a specific marker of pancreatic damage whereas elevation in PLA $_{2}$-IIA are a more general marker of inflammation (Nevalainen et al., 2000). 
${ }_{s P L A}$ has been either found in or produced by various inflammatory cells including platelets (Horigome et al., 1987), mast cells (Murakami et al., 1992), fibroblasts (Shinohara et al., 1992), macrophages (Wightman et al., 1981, Hidi et al., 1993) and neutrophils (Kim et al., 2001). Macrophages isolated from peritoneal exudates of mice and rabbits secrete $\mathrm{PLA}_{2}$ (Wightman et al., 1981). Additionally, sPLA 2 -IIA is constitutively expressed in immune tissues, such as the spleen, thymus, tonsils, and bone marrow (Kramer et al., 1989, Seilhamer et al., 1989). Proinflammatory cytokines such as interferon gamma (IFN- $\gamma$ ), interleukin-1 $\beta$ (IL-1 $\beta$ ), interleukin-6 (IL-6), and tumor necrosis factor- $\alpha$ (TNF- $\alpha)$ can induce its production in a variety of cell types such as human arterial smooth muscle cells, HepG2, HEK293, and BRL-3A in culture (Suga et al., 1993, Kuwata et al., 1998, Peilot et al., 2000). Interestingly the AA release from cells treated with IIA, IID, IIE and V were found to be dependent on IL-1, while treatment with group X released AA irrespective of IL-1 (Kudo and Murakami, 2002). More relevant to the CNS, TNF $\alpha$, IL-1, and lipopolysaccharide (LPS) were shown to induce sPLA $_{2}$-IIA production in cultured astrocytes and direct injection of LPS into brain increased IIA mRNA (Oka and Arita, 1991). Similarly LPS injections have been shown to increase sPLA 2 -IIE production (Murakami et al., 2002b). Consistent with its inducible nature, the promoter region of sPLA 2 -IIA gene contains TATA and CAAT boxes as well as several elements homologous with consensus sequences for binding of transcription factors such as AP-1, C/EBPs, CREB, NF-kB, STAT, and PPAR $\gamma$ (Crowl et al., 1991, Couturier et al., 1999). Finally, sPLA 2 induction can be blocked by anti-inflammatory cytokines, such as platelet-derived growth factor, transforming growth factor $\beta$, and IL- 
10 as well as glucocorticoids (Muhl et al., 1991, Schalkwijk et al., 1992, Touqui and Alaoui-El-Azher, 2001).

Classification, Structure, and Properties of sPLA 2

Eleven mammalian $\mathrm{sPLA}_{2} \mathrm{~s}$ exist which are further divided into groups I, II, III, V, X, XII, and XIII (Fig. 1). All sPLA 2 S are structurally related, and generally are 14-17 $\mathrm{kD}$ secreted enzymes, with six absolutely conserved disulfide bonds, which contribute to the high degree of stability of these enzymes. The enzymes do not have strict fatty acid specificity, unlike cPLA 2 , but instead tend to act on anionic phospholipids in the presence of high concentrations of $\mathrm{Ca}^{2+}$. The central, core protein consists of a highly conserved $\mathrm{Ca}^{2+}$-binding loop (XCGXGG) and a catalytic site (DXCCXXHD) (Kudo and Murakami, 2002). Group differentiations are then made based on the presence or absence of up to two additional unique disulfide bonds, the presence or absence of an $\mathrm{N}$ - or C-terminal extension, and alterations in the conserved catalytic site.

Intracellular Handling

The site of $\mathrm{sPLA}_{2}$ activity has been a point of exhaustive study in recent years. While all $\mathrm{sPLA}_{2}$ isoforms are capable of secretion by definition, recent work has indicated that some heterogeneity exists among their site of activity (Bezzine et al., 2000). Recent studies in CHO and HEK293 cell lines that were stably transfected with human groups IIA and $\mathrm{X}$ have shed light on this subject. Following stimulation by fetal bovine serum and IL-1 $\beta$, both $\mathrm{SPLA}_{2}$-IIA and $\mathrm{X}$ are transcribed within the cell nucleus and synthesized in the endoplasmic reticulum prior to packaging in the Golgi apparatus 
(Mounier et al., 2004). See Fig. 2. This post synthesis packaging most likely results in the perinuclear puncta observed after stimulation, an observation which was previously ascribed to invagination of heparin sulfate proteoglycans (HSPG)-bound $\mathrm{SLA}_{2}$, a process described later. It is within the Golgi apparatus and later microvesicles, prior to initial secretion, that sPLA 2 -IIA is primarily active (Bezzine et al., 2000). The reason for this is that most group II sPLA $\mathrm{SL}_{2}$ as well as $\mathrm{SPLA}_{2}$-IB show a marked preference for anionic phospholipids, such as phosphatidylglycerol, phosphatidylethanolamine and phosphatidylserine, which are generally segregated to the inner leaflet of plasma membranes (Porcellati, 1983). In contrast, $\mathrm{sPLA}_{2}-\mathrm{V}$ and $\mathrm{X}$ can hydrolyze both anionic phospholipids and charged-neutral phosphatidylcholines. These difference in phospholipid preference results in secreted groups I and II having decreased abilities to act on the outer layer of plasma membranes (Bezzine et al., 2000, Murakami and Kudo, 2001). Once secreted, $\mathrm{sPLA}_{2}$ isoforms can then 1) metabolize the external plasma membrane, 2) bind to the $\mathrm{SPA}_{2}$ receptor $\left(\mathrm{sPLA}_{2}-\mathrm{R}\right)$, or 3 ) be internalized by the HSPG shuttle. Again, each of these actions is governed by isoform and species specificity, (see Fig. 2). 
Figure 2. Intracellular Handling and $\mathrm{sLA}_{2}$ Activity

Following stimulation by various cytokines [1], $\mathrm{sPLA}_{2}$ is synthesized in the nucleus [2] and endoplasmic reticulum prior to packaging for secretion in the Golgi apparatus [3]. It is within the Golgi apparatus and later micro vesicles that certain isoforms, particularly IIA, are predominantly active. Following secretion [4], sPLA 2 can metabolize the extracellular lipid membrane directly, bind to the $\mathrm{sPLA}_{2}$ receptor $\left(\mathrm{sLA}_{2}-\right.$ R), and/or be endocytosed via the heparin sulfate proteoglycan shuttle (HSPG shuttle). Of course, each of these actions is governed by species and isoform specificity. The inset shows the general metabolism of phospholipids by $\mathrm{sPLA}_{2}$. $\mathrm{sPLA}_{2}$ first hydrolyzes the acyl bond at the sn-2 position of glycerophospholipids to produce free fatty acids (such as arachidonic acid) and lysophospholipid (Lyso-PL). Arachidonic acid can then be further modified by COX to form prostaglandins, lipoxygenase to form leukotrienes, or cytochrome P450 to form epoxides. Prostaglandins can be further modified to form thromboxanes. These eicosanoids have metabolic activities including proinflammatory and vasoconstrictive functions. 
Extracellular Space

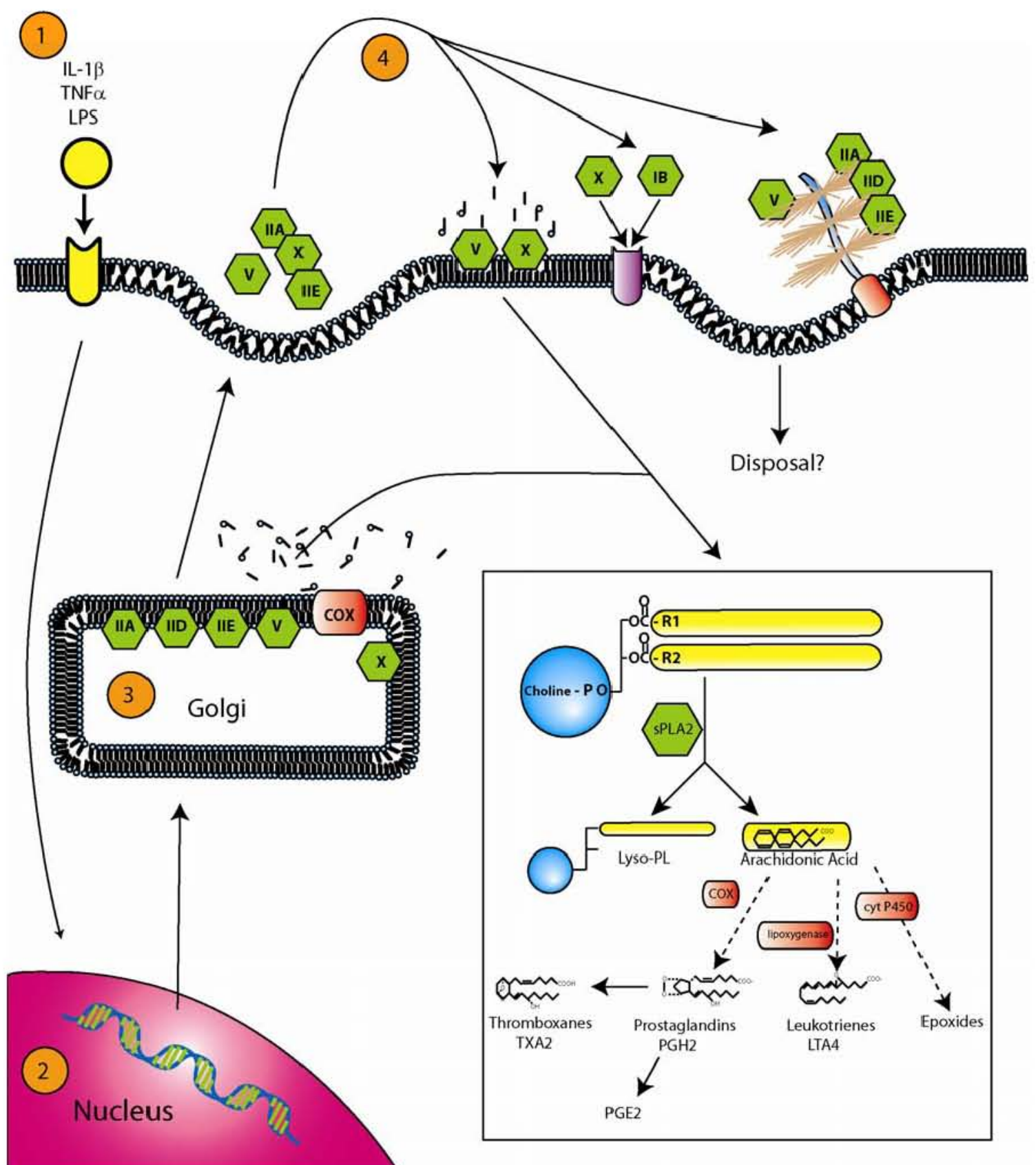




\section{HSPG Shuttling}

Some of the group II subfamily of $\mathrm{SPLA}_{2} \mathrm{~s}$, including IIA, IID, and V are highly cationic and bind tightly to anionic heparinoids such as heparin and heparin sulfate (Murakami et al., 1996, Murakami et al., 1998) (See Fig. 2). Since cell surfaces are usually rich in heparin sulfate proteoglycans (HSPG), significant portions of these ${ }_{s} \mathrm{PLA}_{2} \mathrm{~S}$ are membrane-bound rather than being secreted into the culture media (Murakami et al., 1999b, Murakami et al., 2001). Other sPLA 2 s (IB, IIC, and X) with neutral to acidic pHs show no, or very low, heparinoid affinity and are secreted into the medium. Some authors suggest that this binding facilitates phospholipid digestion since treatment of stably transfected cells with heparinase, exogenous heparin, and GPIspecific phospholipase C, (Murakami et al., 1993, Suga et al., 1993, Kuwata et al., 1998, Murakami et al., 1999a) or mutation of the heparin binding domain in sPLA 2 -IIA (Murakami et al., 1996, Murakami et al., 1998, Murakami et al., 2001) markedly attenuates $\mathrm{SPLA}_{2}$-IIA-mediated prostaglandin generation. In contrast, one recent study found that treatment of cells with heparin had little or no effect on sPLA 2 -IIA activity (Mounier et al., 2004). Also of note, HSPG has been shown to increase following cerebral stab injury with an implicated role in storage, nuclear trafficking, and cellspecific injury responses in CNS wounds (Leadbeater et al., 2006). The closely related chondroitin sulfate proteoglycans are also greatly expressed following SCI (Tang et al., 2003, Chau et al., 2004).

\section{Receptor Binding Domain}


In addition to its enzymatic function, some $\mathrm{SPA}_{2} \mathrm{~S}$ mediate their biological function through a membrane receptor. Two $\mathrm{SPA}_{2}$ receptors have been identified to date, the M-type (Hanasaki and Arita, 1992b, Ishizaki et al., 1994, Lambeau et al., 1994) and the N-type (Lambeau and Lazdunski, 1999), named for their discovery in muscle and neural tissue respectively. However, mammalian $\mathrm{SPLA}_{2} \mathrm{~S}$ only bind to the M-type which was later discovered to be relatively widespread and not merely confined to muscle (Hanasaki and Arita, 1999). Since only the M-type has the ability to initiate an intracellular signal we will restrict our discussion to its properties and further refer to it as $\mathrm{SPLA}_{2}-\mathrm{R}$. The receptor is a type 1 transmembrane glycoprotein with a molecular mass of 180-220 kDa and is a member of the C-type animal lectin family (subgroup VI) (Ohara et al., 1995). The intracellular region consists of approximately 40 amino acids and contains both a consensus sequence for casein-kinase II phosphorylation (Ancian et al., 1995) and a consensus sequence for coated-pit-mediated endocytosis (Ohara et al., 1995). Accordingly, the receptor undergoes internalization after sPLA $\mathrm{A}_{2}$ binding (Hanasaki and Arita, 1992a). Interestingly the isozymes of $\mathrm{SPLA}_{2}$ show varying affinities for the $s P L A_{2}-\mathrm{R}$ in different species. For example, the rabbit sPLA $\mathrm{A}_{2}-\mathrm{R}$ is very promiscuous, binding to almost all sPLA 2 tested to date. The mouse $\mathrm{sPA}_{2}-\mathrm{R}$ on the other hand binds only IB, IIA, and X, while the rat sPLA 2 -R only binds sPLA 2 -IB (Cupillard et al., 1999), (See Fig. 1). Likewise sPLA 2 -IIA does not seem to bind to the sPLA $2-R$ in humans (Cupillard et al., 1999). In general, sPLA 2 -IB and X appear to be the predominant ligand of this receptor (Morioka et al., 2000b) and most of the research has therefore focused on their effects (Ancian et al., 1995, Hernandez et al., 1998, Fonteh et al., 2000). This specificity suggests that the biological function of $\mathrm{sPLA}_{2}-\mathrm{R}$ could vary wildly among 
species. However, the generalized functions of the sPLA $2-\mathrm{R}$ are far reaching including cell growth (Arita et al., 1991), proliferation (Kinoshita et al., 1997), and migration (Kanemasa et al., 1992). It has also been suggested that sPLA $2-R$ functions in the clearance of extracellular sPLA ${ }_{2}$ s to protect against enzymatic over activity, particularly $\mathrm{sPLA}_{2}-\mathrm{X}$ which has potent activity against the extracellular membrane, unlike IB and IIA as stated above (Lambeau and Lazdunski, 1999, Yokota et al., 2001). Additionally, ${ }_{\text {SPLA }}-\mathrm{R}$ knockout mice have significantly reduced levels of TNF $\alpha$ and IL-1 $\beta$ after systemic LPS administration suggesting an inflammatory role (Hanasaki et al., 1997). For an excellent review on the role of the $\mathrm{SPLA}_{2}-\mathrm{R}$ in $\mathrm{SPLA}_{2}$ function, please see (Lambeau and Lazdunski, 1999, Hanasaki and Arita, 2002).

Group I -

${ }_{\mathrm{sPLA}}$-IB was the first $\mathrm{sPLA} 2$ to be discovered and is predominantly present in pancreatic juices (Seilhamer et al., 1986). sPLA $_{2}$-IB lacks a C-terminal extension and is secreted as a catalytically inactive propeptide that is later proteolytically cleaved (Verheij et al., 1981). Group IB has a unique five amino acid extension termed the pancreatic loop in the middle of the molecule as well as a group specific disulfide bond between Cys $^{11}$ and Cys $^{77}$ (Verheij et al., 1981, Seilhamer et al., 1986). sPLA 2 -IB is almost exclusively secreted into the medium of transfected cells (Kudo and Murakami, 2002). Interestingly, sPLA 2 -IB shows low affinity for both heparinoids and phosphatidylcholine (PC) on the external membrane leaflet. Subsequently, it was discovered that sPLA $\mathrm{PL}_{2} \mathrm{IB}$ 
can only release AA indirectly through the M-type $\mathrm{sPLA}_{2}$ receptor-dependent pathway via $\mathrm{cPLA}_{2} \alpha$ activation (Ancian et al., 1995, Hernandez et al., 1998, Fonteh et al., 2000).

Groups II and V-

The second member of the $\mathrm{sPA}_{2}$ family, later named group IIA, was first cloned in 1989 (Komada et al., 1989) and is constitutively expressed in immune tissues, such as the spleen, thymus, tonsils and bone marrow (Kramer et al., 1989, Seilhamer et al., 1989) as well as the digestive system of some mouse strains (Kennedy et al., 1995). sPLA SIA $_{2}$ (Komada et al., 1989, Kramer et al., 1989, Seilhamer et al., 1989), IID (Ishizaki et al., 1999, Valentin et al., 1999), and IIE (Suzuki et al., 2000, Valentin et al., 2000a) are the archetypical group II enzymes. Typically, the enzymes have a C-terminal extension and a disulfide bond linking $\mathrm{Cys}^{50}$ with a Cys in the C-terminus. IIC (Chen et al., 1994) and IIF (Valentin et al., 2000b) have minor variations in amino acids and disulfide structure. Similar to IIA, sPLA $\mathrm{AIIE}_{2}$ is constitutively expressed in human lung and mouse uterus, brain, heart, liver and testis at low levels (Valentin et al., 1999, Suzuki et al., 2000, Murakami et al., 2002b). However, LPS has been shown to induce IIE expression in macrophages, suggesting an inflammatory role as well (Suzuki et al., 2000). sPLA 2 -IIF is highly expressed in the mouse embryo, suggesting a roll in development and it is upregulated by LPS as with other group II enzymes (Valentin et al., 1999, Murakami et al., 2002a). Finally, groups IIA, IIC, IID, IIE, and V all utilize the HSPG-shuttling pathway.

Often considered in the same breath with group II enzymes is group V. Group V shows the highest homology with the group II enzymes and is similarly located on human 
chromosome 1 (1p34-36) (Valentin et al., 2000b). However group V lacks the typical group II C-terminal region, thus justifying its isolation. $\mathrm{sPLA}_{2}-\mathrm{V}$ functions as the primary mouse $\mathrm{SPLA}_{2}$. This is since mice express group $\mathrm{V}$ at higher levels than any of the group II enzymes (Sawada et al., 1999) and since some species of mice have a frame shift mutation resulting in a natural sPLA 2 -II knock out (Kennedy et al., 1995).

However, as with group II, group V is closely linked with inflammation as well being found in mast cells (Sawada et al., 1999), macrophages (Balboa et al., 2003), and type 2 T helper cells (Ho et al., 2001). As with group II, group V is upregulated by LPS (Sawada et al., 1999).

\section{Group X-}

Group X possesses characteristics of both group I and group II enzymes. sPLA $2^{-}$ $\mathrm{X}$ contains the disulfide bonds of both group I and group II as well as the group II, Cterminal extension (Cupillard et al., 1997). Additionally, like group I, it is secreted as a zymogen with cleavage of the N-terminal propeptide for activation (Hanasaki et al., 1999). Like sPLA $2-I B$, cells transfected with group $X$, secrete this $\mathrm{SPLA}_{2}$ almost exclusively into the culture medium rather than having it bound to the membrane like group II enzymes (Kudo and Murakami, 2002). This is not surprising since unlike group II, group X does not readily bind HSPG and shows high activity towards PC, a dominant phospholipid enriched in the outer leaflet of the plasma membrane (Hanasaki et al., 1999, Murakami et al., 1999a, Morioka et al., 2000a, Morioka et al., 2000b, Murakami et al., 2001). Typically, $\mathrm{sPLA}_{2}-\mathrm{X}$ is expressed in the digestive organs such as intestine, colon and stomach and in some immune organs (Cupillard et al., 1997). However, unlike group 
II, group $\mathrm{X}$ is constitutively expressed with little or no change in most tissues (Kudo and Murakami, 2002).

Groups III and XII -

A distinct class of soluble $\mathrm{sPLA}_{2} \mathrm{~s}$, distantly related to groups I and II, were later discovered in bee and lizard venom and are classified as group III. A mammalian homolog of group III was discovered in 2000 (Valentin et al., 2000a) . Groups III and XII only share the $\mathrm{Ca}^{2+}$-binding loop and catalytic site with groups I, II, V, and X. At $55 \mathrm{kDa}$, Group III is considerably large than all the other sPLA 2 isozymes. While maintaining all the $\mathrm{PLA}_{2}$ signature characteristics such as, 10 cystines, the $\mathrm{Ca}^{2+}$-biniding loop, and catalytic site, it additionally has a large $\mathrm{N}$ - and $\mathrm{C}$ - terminal flanking regions that add to its molecular weight (Valentin et al., 2000a). Within humans, sPLA 2 -III was found to be in high abundance in heart, skeletal muscle, liver, and kidney, but had only weak expression in the brain (Valentin et al., 2000a). Group-XII is a much smaller enzyme than group III (19 kDa), lacks an N- or C-terminal flanking regions, and has deviations in the $\mathrm{Ca}^{2+}$ bind loop, that are inconsistent with other sPLA $2 \mathrm{~s}$ (Gelb et al., 2000, Ho et al., 2001). Group XII is expressed in human kidney, heart, and skeletal muscle (Gelb et al., 2000, Valentin et al., 2000a). Relatively little is known about the function of either group III or XII in the mammalian system. 
Suggested Mechanisms underlying sPLA $\mathrm{A}_{2}$-Induced CNS Injury

The sPLA $\mathrm{A}_{2}$ family of enzymes could damage the CNS by multiple injury mechanisms. First, $\mathrm{sPLA}_{2}$ could damage cells directly by attacking cellular membranes and triggering apoptosis. Secondly, the products of lipid metabolism, lysophospholipids and free fatty acids, are detrimental to the CNS and can produce pro-inflammatory mediators, generate free radicals, and increase excitotoxicity (O'Regan et al., 1995b, Farooqui et al., 1997b, Klein, 2000). Interestingly often the injurious agents produced by $\mathrm{SPLA}_{2}$ metabolism in turn initiate synthesis of $\mathrm{SPLA}_{2}$ resulting in a positive feedback loop. See Fig. 3. Our discussion will begin by looking the direct effects of $\mathrm{sPLA}_{2}$ on cells and then how oxidative stress, inflammatory cytokines, and excitotoxic neurotransmitters can each initiate $\mathrm{sPLA}_{2}$ synthesis and be exacerbated by $\mathrm{SPLA}_{2}$ 's presence. 


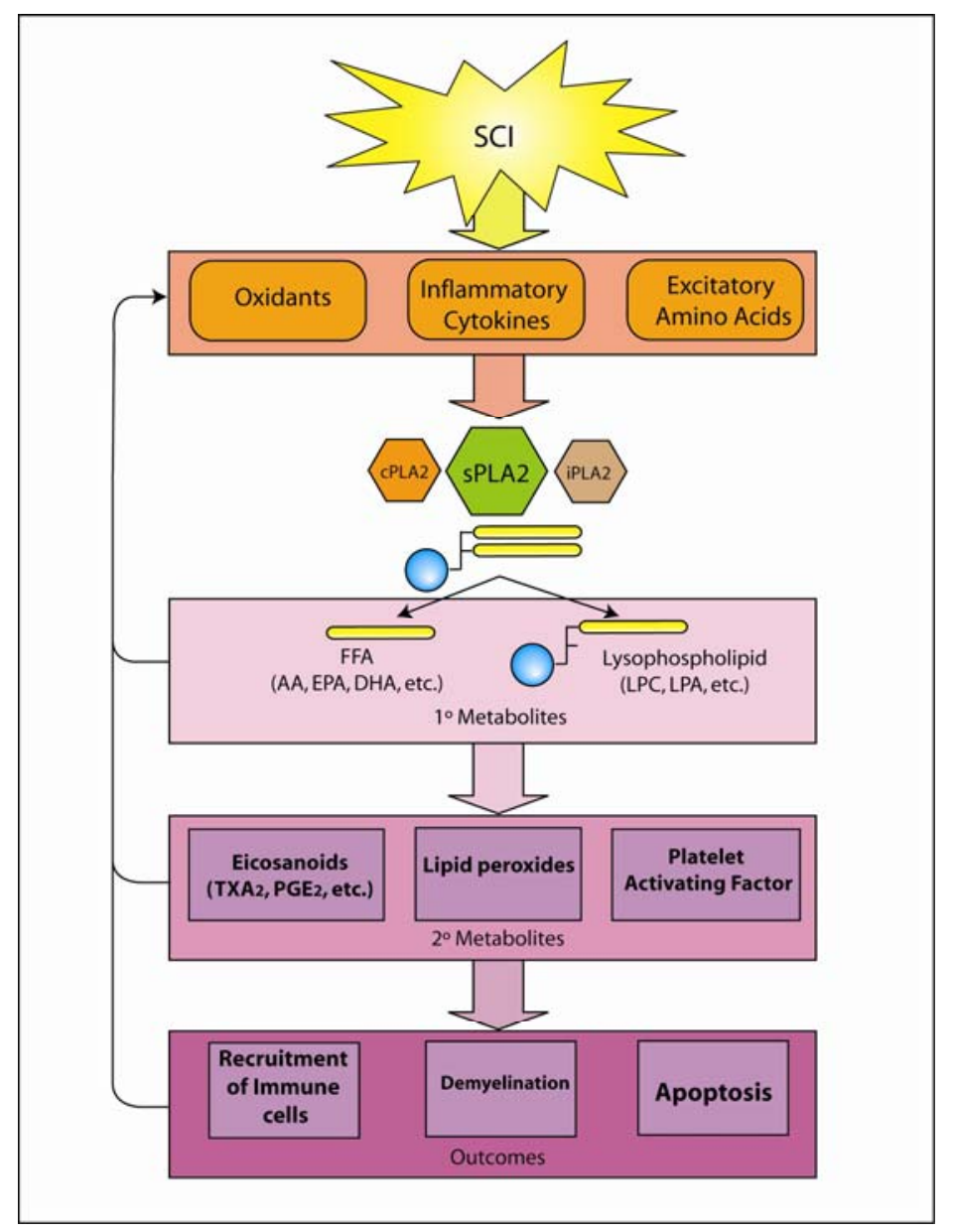

Figure 3. Overview of sPLA 2 's Role in Spinal Cord Injury.

The toxicity of $\mathrm{sPLA}_{2}$ is compounded by three factors. 1) $\mathrm{sPLA}_{2}$ is upregulated by commonly known neurotoxic mechanisms such as oxidative stress, cytokines, and EAA. 2) Both the primary metabolites of $\mathrm{PLA}_{2}$ activity, such as free fatty acids and lysophospholipids, and the secondary metabolites, such as eicosanoids and platelet activating factor, are toxic to the CNS. 3) Finally, $\mathrm{sPLA}_{2}$ has been shown to reciprocally upregulate oxidative stress, cytokines, and EAA thus propagating a positive feedback loop resulting in cytotoxicity and secondary SCI. It must also be noted that $\mathrm{SPLA}_{2}$ does not work in isolation from $\mathrm{cPLA}_{2}$ and $\mathrm{PLA}_{2}$, rather a reciprocal activity is often demonstrated among the $\mathrm{PLA}_{2}$ subfamilies. 
Membrane Breakdown and Metabolites

Phospholipids are the main components of the neural cell bi-layer membrane. In addition, they provide the membrane with the necessary environment, fluidity, and ion permeability that are required for the proper function of integral membrane proteins, receptors, and ion channels. $\mathrm{PLA}_{2}$ directly hydrolyses phospholipids resulting in membrane breakdown. This results in alterations of membrane function such as fluidity and permeability, behavior of transporters and receptors, and ion homeostasis, and can eventually lead to functional failure of excitable membranes (Farooqui et al., 1997b, Klein, 2000, Farooqui et al., 2004).

In addition to the direct effects of membrane breakdown on cell survival, the products of sPLA2 enzymatic, activity also exhibit neurotoxic profiles. $\mathrm{sPLA}_{2}$ cleaves phospholipids into the primary metabolites free fatty acid, such as AA, and lysophospholipids such as lysophosphatidyl choline (LPC, a.k.a. lysolechithin). See insert of Fig. 2 and Fig. 3. Both of these agents have been shown to create injury and cytotoxicity in the CNS. AA induces oxidative injury and death in cultured spinal cord neurons (Toborek et al., 1999). AA can later form epoxides via the cytochrome P450 pathway, leukotrienes via the lipoxygenase pathway, or thromboxanes or prostaglandins via the COX pathway. See insert of Fig. 2. Many of these products, such as prostaglandin $\mathrm{E}_{2}\left(\mathrm{PGE}_{2}\right)$ can subsequently act as potent chemoattractants increasing endogenous immune responses and subsequent secondary damage. Additionally, the expression of eicosanoids, such as thromboxane $\mathrm{A}_{2}\left(\mathrm{TXA}_{2}\right)$ and $\mathrm{PGE}_{2}$ following SCI have been linked to trauma induced ischemia (Tonai et al., 1999). LPC produced by sPLA $_{2}$-induced hydrolysis is also implicated in CNS damage (Dutta et al., 1979). 
Interestingly, direct LPC injection has been shown to create a demyelination and infiltration of macrophages in the spinal cord, brain, and peripheral nervous system with a later remyelination by Schwann cells, the myelinating cells of the peripheral nervous system (Blakemore et al., 1977, Blakemore, 1978, Blakemore, 1982, Jeffery and Blakemore, 1995). This demyelination and remyelination by Schwann cells mimics that produced by direct injection of $\mathrm{sPLA}_{2}$, however, $\mathrm{sPLA}_{2}$ has the added cost of severe axonopathy and death of oligodendrocytes prior to remyelination (Titsworth et al., 2007). It has been hypothesized that LPC may mediate the demyelinating effects of $\mathrm{SPLA}_{2}$ injections. Additionally, a recent study by Lauber, et al., found that LPC, generated by iPLA 2 , was the main chemoattractant for monocytic cells and primary macrophages released by apoptotic cells thus facilitating the efficient phagocytosis of cellular debris (Lauber et al., 2003).

Apoptosis

In recent years, apoptosis has been identified as an important mechanism of cell death in many neurological disorders including SCI. Cells undergoing apoptosis generally release free fatty acids including AA, which parallels the reduction in cell viability (Taketo and Sonoshita, 2002, Balsinde et al., 2006), suggesting the involvement of $\mathrm{PLA}_{2}$ in apoptosis. Recently, sPLA $-\mathrm{IB}$, IIA, and III have all been shown to induce neuronal apoptosis (Yagami et al., 2002a, Yagami et al., 2002b, Yagami et al., 2002c, DeCoster, 2003). In contrast, recombinant $\mathrm{SLA}_{2}$ appears to prevent apoptosis of mast cells (Taketo and Sonoshita, 2002). 


\section{Oxidative Stress}

Oxidative injury is a common pathological mechanism in neurological disorders. Free radicals induce not only lipid peroxidation of neural membranes, but also the oxidation of proteins, RNAs, and DNAs. Reactive oxygen species (ROS) including hydrogen peroxide $\left(\mathrm{H}_{2} \mathrm{O}_{2}\right)$ and superoxide radicals are produced by a number of cellular oxidative and metabolic processes including metabolism of AA. PLA 2 metabolism of phospholipids is a well-established source of ROS (Adibhatla and Hatcher, 2006, Nanda et al., 2007). Application of pathophysiological concentrations of free fatty acids has been demonstrated to induce oxidative injury to cultured spinal cord neurons (Toborek et al., 1999). Microinjections of $\mathrm{PLA}_{2}$ into the normal spinal cord induced expression of 4hydroxynonenal, a product of lipid peroxidation and marker for oxygen free radicalmediated membrane injury (Liu et al., 2006). Nethery, et al., (Nethery et al., 1999) found that ROS production in contracting muscle required the presence of $\mathrm{SPLA}_{2}$ but not cPLA $\mathrm{A}_{2}$ or iPLA $A_{2}$. In the presence of 15-lipoxygenase the addition of sPLA $\mathrm{P}_{2}$-IIA or IB greatly enhances the accumulation of hydroperoxides of oxidized free fatty acids (Neuzil et al., 1998). Finally, it can be assumed that the proinflammatory effects of sPLA 2 would result in an immigration of immune cells, which will release copious amounts of ROS (Blight, 1985, Popovich et al., 1997, Park et al., 2004, Jones et al., 2005a, Fleming et al., 2006, Wang et al., 2006). For a review of the ROS species produced by the enzymatic effects of $\mathrm{SPLA}_{2}$ please see (Nanda et al., 2007).

While $\mathrm{sPLA}_{2}$ production of ROS is well established, the induction of $\mathrm{sPLA}_{2}$ by ROS is an immerging concept. One study found that mesangial cells treated with $\mathrm{H}_{2} \mathrm{O}_{2}$ utilized both cPLA 2 and $\mathrm{sPLA}_{2}$ during AA release and that elimination of sPLA $\mathrm{A}_{2}$ greatly 
inhibited AA release (Han et al., 2003), thus suggesting the first of many positive feedback loops.

Inflammation and Inflammatory Cytokines

Inflammation has been implicated in many CNS neurological disorders including SCI. PLA $A_{2}$ may serve as a key molecule that controls the biosynthesis of several wellknown bioactive mediators of inflammation such as eicosanoids (prostaglandins, thromboxanes, leukotrienes and lipoxins) and PAF in a rate-limiting manner (Farooqui et al., 1997b, Farooqui et al., 1999a). In addition, our recently findings showed that sPLA 2 induced expression of cytokines including TNF- $\alpha$ and IL-1 $\beta$ in the injured spinal cord (Liu et al., 2006). On the other hand, there is also strong evidence to suggest that cytokines upregulate $\mathrm{SLA}_{2}$-IIA in vitro and in vivo. sPLA $_{2}$-IIA is up-regulated by TNF$\alpha$ and IL- $1 \alpha / \beta$ after transient focal cerebral ischemia in rats (Adibhatla and Hatcher, 2007). $\mathrm{SPLA}_{2}$-IIA mRNA is expressed in cultured astrocytes and can be induced in response to proinflammatoy cytokines TNF $\alpha$, IL-1 $\beta$, and, INF $\gamma$ (Oka and Arita, 1991, Tong et al., 1995, Li et al., 1999, Xu et al., 2003). Astrocytes isolated from C57Bl/6 mice, which lack the sPLA2-IIA gene, were less responsive to cytokines in the production of $\mathrm{PGE}_{2}$ than were astrocytes expression sPLA 2 -IIA (Xu et al., 2003). Additionally, IL$1 \beta$ and TNF $\alpha$ can also activate COX-2 continuing the proinflammatory pathways (Kuwata et al., 1998, Murakami et al., 1999b, Sawada et al., 1999, Morioka et al., 2000a, Morioka et al., 2002). sPLA 2 also induced AA release and COX-2 expression in cultured neurons independent of other cytokines (Kolko et al., 2003). 


\section{Excitatory Neurotoxicity}

Elevated levels of excitatory amino acids (EAA) have been implicated in the pathogenesis of neural injury and death in many disorders and evidence suggests that ${ }_{\mathrm{sPLA}}$ may stimulate the release of EAA following CNS trauma. First, injections of $\mathrm{sPLA}_{2}$ into the brain caused epileptic seizures and neurotoxicity in vivo (Dorandeu et al., 1998). Secondly, application of $\mathrm{sPLA}_{2}$ to the rat ischemic cerebral cortex resulted in a significant increase in EAA levels and inhibition with mepacrine, a global PLA 2 inhibitor, significantly decreased the ischemia-evoked efflux of EAA into cortical superfusates (O'Regan et al., 1995a). Group IIA sPLA 2 stimulates exocytosis and neurotransmitter release in pheochromocytoma-12 cells and cultured rat hippocampal neurons (Wei et al., 2003). sPLA 2 -induced neuronal death was blocked by MK-801, an N-methyl-D-aspartic acid (NMDA) receptor antagonist, both in vitro and in vivo (Kolko et al., 2002). Finally, administration of the nonselective PLA 2 inhibitor, 4bromophenacyl bromide, inhibited glutamate release in the spinal cord (Sundstrom and Mo, 2002). Only one study so far has suggested that excessive EEA concentrations induce $\mathrm{SPLA}_{2}$ levels in the CNS. Interocerebroventricular injection of kainic acid (KA) resulted in an increase in both total $\mathrm{PLA}_{2}$ and $\mathrm{SPLA}_{2}$ activity and this activity could be blocked by a synthetic short inhibitor peptide for sPLA2-IIA (Thwin et al., 2003).

The exact mechanism of $\mathrm{sPLA}_{2}$ action on EAA release remains unknown. It has been suggested that $\mathrm{PLA}_{2}$ disrupts an artificial planar lipid bilayer in a $\mathrm{Ca}^{2+}$-dependent manner (O'Regan et al., 1996). Matsuzawa demonstrated the role of sPLA 2 -IIA in EEA release in a set of elegant experiments. First, $\mathrm{sPLA}_{2}$-IIA was detected in purified brain

synaptosomes. Secondly, sPLA 2 -IIA was released upon depolarization of neurons with 
either high concentrations of potassium or neurotransmitters. Third, addition of sPLA $_{2}-$ IIA to cultures triggered neurotransmitter release. Finally, $\mathrm{sPLA}_{2}$-IIA inhibition suppressed neurotransmitter secretion (Matsuzawa et al., 1996). The role of $\mathrm{SPLA}_{2}$ in neurotransmitter release is further supported by the fact that exogenously added $\mathrm{SPLA}_{2}$ paralyzed neuromuscular junction preparations by inducing total neurotransmitter release (Rigoni et al., 2005). Interestingly, an equimolar mixture of lysophospholipids and fatty acids closely mimicked the sPLA 2 paralysis (Rigoni et al., 2005). These studies further suggest that changes in the local lipid composition within the synaptic buton trigger neurotransmitter release which would lead to excitotoxicity (Piomelli et al., 2007). Inhibitors of total $\mathrm{PLA}_{2}$ activity such as 4-bromophenacyl bromide, a nonselective $\mathrm{PLA}_{2}$ inhibitor, 7,7-dimethyleicosadienoic, a sPLA 2 specific inhibitor, AACOCF3, a cPLA 2 specific inhibitor, and HELSS, an iPLA 2 specific inhibitor, all reduced the efflux of both

glutamate and aspartate in vivo suggesting the involvement of multiple isoforms of $\mathrm{PLA}_{2}$ in EAA release not merely sPLA 2 (Phillis and O'Regan, 1996).

\section{Role of sPLA 2 in CNS Disorders}

Localization within the CNS

$\mathrm{sPLA}_{2} \mathrm{~S}$ are present in all regions of the mammalian brain. The highest $\mathrm{SPLA}_{2}$ activities are found in medulla oblongata, pons, and hippocampus; moderate activities in the hypothalamus, thalamus, and cerebral cortex; and lowest activities in the cerebellum and olfactory bulb (Thwin et al., 2003). Molloy, et al., utilizing RT-PCR found that mRNAs for sPLA $\mathrm{PLA}_{2}$-IIA and IIC were expressed in all regions of normal rat brain, $\mathrm{sPLA}_{2}-$ 
V was found at low levels in most areas of the brain, but at very high levels in the hippocampus, and sPLA $\mathrm{SL}_{2}$-IB was not detected in the rat brain at all (Molloy et al., 1998). In contrast, Kolko, et al., reported that sPLA $\mathrm{S}_{2}$-IB mRNA was detected in the rat and human brain at very high levels as well as in neurons in primary cultures using various detection methods. The distribution of $\mathrm{SPLA}_{2}$-IB seems to be mainly neuronal, with the highest abundance occurring in the cerebral cortex and hippocampus (Kolko et al., 2005). sPLA 2 -IIA and $\mathrm{V}$ were also detected in the rat cerebellum using immunostaining and in situ hybridization methods (Shirai and Ito, 2004). $\mathrm{sPLA}_{2}$-IIA is associated with the endoplasmic reticulum in perinuclear regions of Purkinje cell somata and $\mathrm{SPLA}_{2}-\mathrm{V}$ was localized in Bergmann glia cells (Shirai and Ito, 2004). Recently, Kolko, et al., found the presence of sPLA2-IIE, $\mathrm{V}$, and $\mathrm{X}$ in the rat brain as well as in neurons in primary cultures using RT-PCR, in situ hybridization, and immunohistochemistry (Kolko et al., 2006). The distribution of sPLA 2 -IIE, $\mathrm{V}$, and $\mathrm{X}$ seems to be mainly neuronal, with the highest abundance occurring in the cerebral cortex and hippocampus (Kolko et al., 2006). In the spinal cord, $\mathrm{SLA}_{2}$ activity was detected in the normal rat spinal cord homogenate (Svensson et al., 2005). Western blots revealed that sPLA2-IIA and V are expressed in the normal rat spinal cord (Svensson et al., 2005). mRNAs of sPLA 2 s (IB, IIA, IIC and V) are also detected in the normal rat spinal cord with RT-PCR (Lucas et al., 2005).

As stated above, the presence of $\mathrm{SPLA}_{2}$ in the CNS, particularly neurons, appears to be both constitutive (groups IB, IIA, V, and X) and inducible (group IIA). While it is assumed that the inducible sPLA $\mathrm{A}_{2}$ expression is associated with inflammation, the normal physiological role of constitutively expressed $\mathrm{SLA}_{2}$ is believed to play a crucial role in exocytosis of synaptic vesicles (Matsuzawa et al., 1996, Phillis and O'Regan, 2004). 
Support for this theory arises from studies by Matsuzawa, et al., in which differentiated PC12 cells were shown to release $\mathrm{SLA}_{2}$-IIA after depolarization and that inhibition of IIA resulted in decreased catecholamine secretion (Matsuzawa et al., 1996). Additionally, snake $\mathrm{PLA}_{2}$ neurotoxins cause paralysis of neuromuscular junctions by triggering a massive release of all presynaptic vesicles (Rigoni et al., 2005). Finally, the concentration of group V in the hippocampus and studies by Chabot, et al., indicate that ${ }_{s P L A}-\mathrm{V}$ may play a role in the regulation of long-term potentiation and long-term depression (Chabot et al., 1998).

\section{Spinal Cord Injury}

Damage from acute SCI occurs in two phases, an initial mechanical injury followed by a secondary injury mediated by multiple processes including inflammation, free radical induced cell death, cytokine production, and glutamate excitotoxicity (Hall and Braughler, 1986, Young, 1993, Buki et al., 2000, Park et al., 2004). Following SCI, inflammatory cells such as polymorphonuclear neutrophils, macrophages, and lymphocytes quickly infiltrate into the traumatized cord, and flood the interstitial tissue with proinflammatory cytokines such as TNF- $\alpha$ and IL-1 $\beta$, and neurotoxic factors from leukocytes such as nitric oxide, hydrogen peroxide, and myeloperoxidase (Blight, 1985, Popovich et al., 1997, Park et al., 2004, Jones et al., 2005a, Fleming et al., 2006, Wang et al., 2006). Free radical generation and lipid peroxidation were also found to be early events subsequent to SCI (Hall and Braughler, 1986, Hall et al., 1992, Springer et al., 1997). EAAs such as glutamate and aspartate are released rapidly following SCI and their extracellular concentrations increased to neurotoxic levels within minutes post SCI (Panter et al., 1990, Farooque et al., 1996, Liu et al., 1999b, McAdoo et al., 1999). The 
interplay between multiple harmful substances likely perpetuates the progressive course of secondary injury, resulting in cell death, axonal destruction, demyelination and functional loss. Evidence suggests that these harmful substances generated in the injured cord might induce $\mathrm{SPLA}_{2}$ expression which in turn exacerbates SCI. This hypothesis is supported by in vitro and in vivo experiments that indicate that increases in $\mathrm{PLA}_{2}$ activity and its metabolic products can in turn exacerbate inflammation (Bonventre, 1996, Murakami et al., 1997), oxidation (Bonventre, 1996, Murakami et al., 1997), demyelination (Titsworth et al., 2007), and neurotoxicity (Clapp et al., 1995, Bonventre, 1996) suggesting that $\mathrm{SPLA}_{2}$ may serve as a common mediator in the progression of secondary SCI. See Fig. 4. 


\section{Figure 4. sPLA $A_{2}$ Activity Within Spinal Cord Injury.}

Following SCI, oxidative stress, cytokines, and EAA are upregulated. These toxic factors then up regulate the synthesis of $\mathrm{sPLA}_{2}$. Subsequently sPLA 2 mediates the hydrolysis of phospholipids into lysophospholipids (Lyso-PL), such as LPC, and free fatty acids (FFA), such as AA. Independent of other factors, $\mathrm{sPLA}_{2}$ and LPC demyelinate axons in the spinal cord and $\mathrm{sPLA}_{2}$ and AA have been shown to trigger apoptosis in neurons and oligodendrocytes. The metabolism of AA results in increased oxidative stress from lipid peroxidation and increased eicosanoids which have been shown to increase inflammation and ischemia. LPC also increases inflammation while its metabolite, PAF, triggers ischemia. Infiltrating polymorphonuclear neutrophils (N), lymphocytes $(\mathrm{L})$, and macrophages $(\mathrm{M} \phi)$ then flood the CNS with more sPLA 2 , oxidants, and cytokines thus exacerbating the positive feedback loop, while the upregulation in $\mathrm{sPLA}_{2}$ and LPC trigger the release of EAAs from synaptic terminals. 


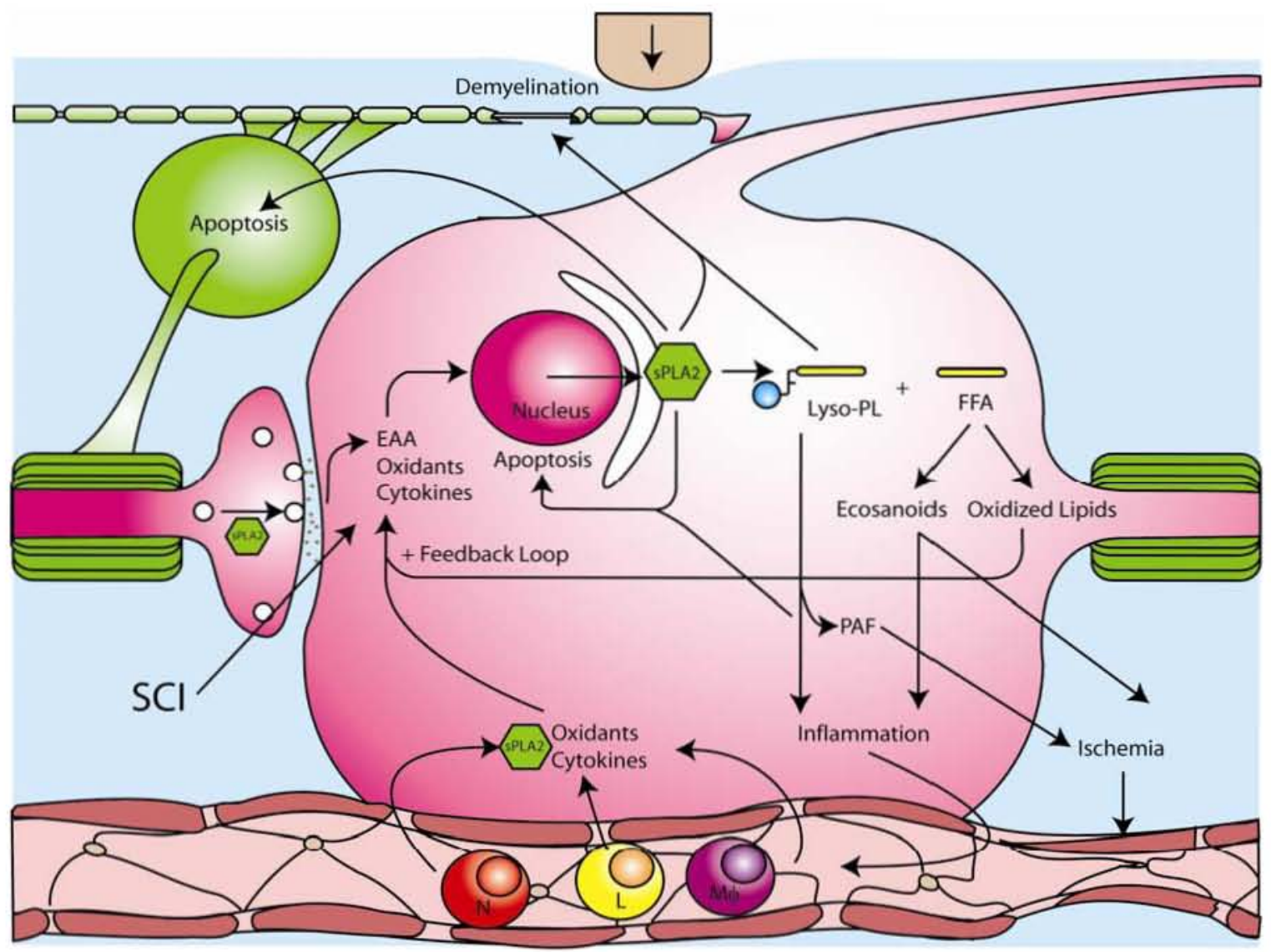


Recently, we showed that PLA 2 activity increased following SCI, peaking at $4 \mathrm{~h}$ post injury, and remaining elevated for one week. In the same study, we found that cPLA 2 expression did not peak until 7 days post injury (Liu et al., 2006). In vitro experiments showed that both sPLA 2 and melittin, an activator of endogenous $\mathrm{PLA}_{2}$, induced spinal neuronal death in a dose-dependent manner, an effect that could be substantially reversed by mepacrine, a PLA 2 inhibitor (Liu et al., 2006). Similarly direct injection of $\mathrm{SPLA}_{2}$ also lead to demyelination that could be effectively attenuated with mepacrine, an inhibitor of all $\mathrm{PLA}_{2}$ isoforms (Liu et al., 2006). Injections of $\mathrm{sPA}_{2}$ also induced the expression of inflammatory cytokines TNF- $\alpha$ and IL-1 $\beta$, as well as 4hydroxynonenal, a product of lipid peroxidation and a marker for oxygen free radicalmediated membrane injury (Liu et al., 2006). Indeed, in vivo and in vitro experiments show that exogenous administration of $\mathrm{sPLA}_{2}$ can induce neuronal death, oligodendrocyte death, and tissue damage (Clapp et al., 1995, Kolko et al., 1996, Kolko et al., 1999, Yagami et al., 2002a, Yagami et al., 2002b, Yagami et al., 2002c, Liu et al., 2006, Titsworth et al., 2007). Importantly, no study to date has directly observed whether $\mathrm{sPLA}_{2}$ mRNA or protein expression increases following SCI.

The induction of $\mathrm{SPLA}_{2}$ following SCI is supported by the fact that the substrate of $\mathrm{PLA}_{2}$ metabolism, phospholipids, decreases acutely following SCI. There is a dramatic loss of membrane phospholipids following CNS trauma. During the first minute of compression trauma to the spinal cord, $10 \%$ of the plasmenylethanolamine is reduced with an overall loss of $18 \%$ found at 30 min post compression injury (Horrocks et al., 1985). The hydrolysis of membrane phospholipids by $\mathrm{PLA}_{2}$ is a rate-limiting step 
for generation of proinflammatory mediators, eicosanoids, and PAF (Farooqui et al., 1997b, Farooqui et al., 1999b).

Additionally, there is an increase in free fatty acids, eicosanoids, lipid peroxides, and lysophospholipids following SCI (Demediuk et al., 1985, Demediuk et al., 1989, Lukacova et al., 1996). Severe trauma was associated with biphasic increases in free fatty acids levels, with levels peaking at $15 \mathrm{~min}$ and $24 \mathrm{hr}$ post-trauma before declining over the next 6 days (Demediuk et al., 1989). Within the first few minutes of SCI, free fatty acids have increased in the grey matter and later increase within the white matter suggesting acute PLA 2 activity (Demediuk et al., 1985, Faden et al., 1987, Demediuk et al., 1989). The production of free fatty acid represents a source of potentially dangerous ROS by initiating lipid peroxidation. Hydroxyl radicals can attack polyunsaturated fatty acids in membrane glycerophospholipids forming peroxyl radicals and propagating the chain reaction of lipid peroxidation products (Muralikrishna Adibhatla and Hatcher, 2006, Sun et al., 2007). The generation of free fatty acids in SCI is closely associated with increases in free radical formation observed in the lesion of the injured spinal cord (Hamada et al., 1996, Azbill et al., 1997). Application of pathophysiological concentrations of free fatty acids has been demonstrated in vitro to induce oxidative injury in spinal cord cell cultures (Toborek et al., 1999). The neurotoxic effects of AA have also been observed in hippocampal neurons and cortical neurons (Li et al., 1997) as well as oligodendrocytes (Wang et al., 2004).

Later products of free fatty acid metabolism also increase within the injured spinal cord. COX, also known as prostaglandin $\mathrm{G} / \mathrm{H}$ synthase, is the rate-limiting step in the production of prostaglandins. See insert of Fig. 2. COX-2 mRNA and protein expression 
are increased from 2 to $48 \mathrm{hr}$ following SCI and the selective inhibition of COX-2 results in histological and functional sparing as assessed by the Basso, Beattie, and Bresnahan locomotion score (Resnick et al., 1998, Hoffmann, 2000, Schwab et al., 2000, Hains et al., 2001). COX-1 has also been shown to increase following SCI, persisting for as long as 4 weeks (Schwab et al., 2000). This upregulation in COX in the presence of free fatty acids, such as AA, logically progresses to an upregulation of eicosanoids. Bioactive eicosanoids, derived from $\mathrm{PLA}_{2}$-induceds production of AA, have been implicated as mediators of secondary injury via a host of mechanisms. The expression of eicosanoids, such as $\mathrm{TXA}_{2}$ and $\mathrm{PGE}_{2}$ increased in the injured cord tissue within hours of SCI and their vasoactive properties are thought to create microemboli in addition to $\mathrm{PGE}_{2}$ 's well known pro-inflammatory effects (Tonai et al., 1999, Resnick et al., 2001). Increased production of TXA $2, \mathrm{PGI}_{2}, \mathrm{LTC}_{4}$ and 5-HETE has also been confirmed in experimental SCI (Jacobs et al., 1987, Mitsuhashi et al., 1994). PGF $_{2 \alpha}$ increases three fold following SCI, and when exogenously added caused significant cell loss, increased hydroxyl radicals, and malondialdehyde - an end product of membrane lipid peroxidation (Liu et al., 2001).

The effect of lysophospholipids on spinal cord tissue has been extensively studied and lysophospholipids such as LPC and its later metabolites, such as PAF, are metabolically active in the CNS. For over 30 years it was known that injections of LPC into the spinal cord causes demyelination (Blakemore et al., 1977, Blakemore, 1978, Blakemore, 1982, Toborek et al., 1999) as well as expression of a number of chemokines and cytokines, similar to those produced following SCI (Ousman and David, 2000, Ousman and David, 2001). While lysophospholipid levels following SCI or traumatic brain injury (TBI) have not been assessed directly, their presence is strongly implied from 
the generous production of free fatty acids and a decrease in phospholipids. PAF, a metabolite of lysophospholipids, increases 20 -fold after SCI induced by stroke (Lindsberg et al., 1990, Faden and Halt, 1992, Xiao et al., 1995, Xiao et al., 1996, Hostettler and Carlson, 2002). Intrathecal administration of PAF leads to reduced spinal cord blood flow and motor deficits, an effect which can be blocked by the PAF receptor antagonist, WEB 2170 (Faden and Halt, 1992). Treatment with WEB 2170 after acute spinal cord contusion resulted in significant increases in white matter sparing as well as decreases in proinflammatory cytokine mRNA levels within the lesion epicenter (Hostettler and Carlson, 2002, Hostettler et al., 2002). Treatment with the PAF receptor antagonist BN52021 also improves behavioral function after SCI (Xiao et al., 1998). In vitro experiments showed that low concentrations of PAF resulted in neuronal differentiation and sprouting, while higher concentrations were neurotoxic (Kornecki and Ehrlich, 1988). PAF-induced death of not only cultured neuronal cells in a concentration -dependent manner (Xu and Tao, 2004, Bate et al., 2007) but also that of oligodendrocytes and astrocytes (Hostettler et al., 2002).

Oxidative stress is well established following SCI (Liu et al., 1999a, Liu et al., 2001, Liu et al., 2003, Bao and Liu, 2004, Liu et al., 2004a). Work by Liu et al., has shown that $\mathrm{H}_{2} \mathrm{O}_{2}$ (Liu et al., 1999a), iron (Liu et al., 2004a), and hydroxyl radicals (Liu et al., 2004a) are formed following SCI. Furthermore pathophysiological doses of these oxidants administered exogenously in vivo created significant cell death at $24 \mathrm{hr}$ that could be blocked by a broad spectrum reactive species scavenger (Liu et al., 2004a). Administration of $\mathrm{PGF}_{2 \alpha}$ resulted in a 3-fold increase in hydroxyl radicals and a 2-fold increase in malondialdehyde, an end product of membrane lipid peroxidation (Liu et al., 
2001). It has also been shown that $\mathrm{H}_{2} \mathrm{O}_{2}$ is toxic to neurons (Whittemore et al., 1994, Hoyt et al., 1997, Samanta et al., 1998, Lim et al., 2002, Fatokun et al., 2007), astrocytes (Rouach et al., 2004, de Almeida et al., 2007), and oligodendrocytes (Richter-Landsberg and Vollgraf, 1998, Vollgraf et al., 1999, Mronga et al., 2004). Oxidative stressors, such as $\mathrm{H}_{2} \mathrm{O}_{2}$ administration, also increase AA release in neurons and mesangial cells (Samanta et al., 1998, Han et al., 2003). It has recently been suggested that generation of ROS and polyunsaturated fatty acids, via cPLA 2 , following CNS injury mediates NF- $\mathrm{kB}$ migration from the cytosolic to the nucleus where it induces gene expression of $\mathrm{sPLA}_{2}$ and other lipid enzymes, thus potentiates a positive feedback loop (Sun et al., 2007).

High levels of EAAs such as glutamate and aspartate in experimental SCI are also an important mechanism inducing secondary injury (Park et al., 2004). Growing evidence indicates that $\mathrm{SPLA}_{2}$ could mediate EAA-induced neuronal death and tissue damage. Marked increases in $\mathrm{PLA}_{2}$ activity and AA release have been reported after treatments of neuronal cultures with glutamate, NMDA and KA) (Dumuis et al., 1988, Farooqui et al., 2001). In addition, glutamate release in the spinal cord can be suppressed by $\mathrm{PLA}_{2}$ inhibitors such as indomethacin by $40 \%, \mathrm{AACOCF}_{3}$ by $45 \%$, and 4-bromophenacyl bromide by $36 \%$, suggesting that increased $\mathrm{PLA}_{2}$-mediated EAA release is part of a positive feedback mechanism (Sundstrom and Mo, 2002). Additionally, application of $\mathrm{SPLA}_{2}$ to the ischemic rat cerebral cortex resulted in a significant increase in EAA levels and a general PLA $\mathrm{A}_{2}$ inhibitor mepacrine significantly decreased the ischemia evoked efflux of EAA into cortical superfusates (O'Regan et al., 1995a). Thus, the excessive stimulation of NMDA receptors, as occurs in the spinal cord trauma, may result in 
stimulation of $\mathrm{sPLA}_{2}$ activity leading to alterations in membrane composition, permeability, and fluidity leading to neuronal and glial cell death.

In summary, $\mathrm{sPLA}_{2}$ can be induced by several key injury mediators such as inflammatory cytokines, free radicals, and EAAs that have been shown to increase following traumatic SCI. Furthermore, this increase in $\mathrm{SLA}_{2}$ activity can further increase inflammation, oxidation, and EAA release. This indicates that $\mathrm{SPA}_{2}$ activation may play a central role in a positive feedback loop triggered by traumatic SCI resulting in neuronal and glial cell death, tissue damage, and corresponding behavioral impairments. If $\mathrm{SLA}_{2}$ is present and increased following $\mathrm{SCI}$ then it may act as a convergence molecule that mediates multiple key mechanisms of secondary spinal cord injury and blocking $\mathrm{SPLA}_{2}$ action may represent a novel and efficient strategy to block multiple injury mechanisms.

Brain Injury

Similar to SCI, TBI also triggers secondary or delayed cell death by multiple injury processes including ischemia, inflammation, generation of free radicals, and glutamate release, all of which have been showed to induce PLA 2 activity (Phillis and O'Regan, 2004, Mattson, 2005, Farooqui et al., 2006). Like SCI, there is clear evidence that TBI induces $\mathrm{PLA}_{2}$ activity resulting in membrane phospholipid degradation, generation of proinflammatory mediators, such as eicosanoids and PAF, formation of free radicals, and subsequent lipid peroxidation. Following closed head injury in rats, total $\mathrm{PLA}_{2}$ activity increased (Shohami et al., 1989). Additionally, after open traumatic brain injury, free fatty acids, such as AA, were released and membrane phospholipid 
degradation was found (Dhillon et al., 1994, Homayoun et al., 1997, Homayoun et al., 2000). In humans, an increase in free fatty acids in cerebrospinal fluid (CSF) following brain injury has been reported (Pilitsis et al., 2003).

No report to date has investigated the expression of $\mathrm{SPLA}_{2}$ following traumatic brain injury either, however, $\mathrm{cPLA}_{2}$ and 4-hydroxynonenal were expressed in the transected brain ( $\mathrm{Lu}$ et al., 2001). Additional reports have confirmed the presence of down stream metabolites of AA. Pronounced increases in prostaglandin $F_{2 \alpha}$, prostaglandin $\mathrm{D}_{2}$, leukotrienes, and thromboxane $\mathrm{B}_{2}$ have all been reported to occur in brain tissues after KA injection (Baran et al., 1987) and increases in $\mathrm{PGE}_{2}$ following closed head injury (Shohami et al., 1989).

Conditions that increase $\mathrm{PLA}_{2}$ have been shown following TBI, just as in SCI. Cerebral penetration and contusive injury both increase oxidative stress in the brain (Layton et al., 1997, Kline et al., 2004) and blockade of oxidative stress increases learning and histological sparing (Kline et al., 2004). Under both experimental and clinical settings, the level of extracellular EAAs such as glutamate and aspartate increased following TBI (Faden et al., 1989; Palmer et al., 1993; Globus et al., 1995; Bullock et al., 1998). Additionally, both competitive and noncompetitive NMDA and non-NMDA receptor antagonists are efficacious in the treatment of experimental brain injury (Bullock and Fujisawa, 1992). Several studies showed that glutamate, NMDA, and KA result in a dose-dependent increase in AA release in hippocampal neuronal cultures (Dumuis et al., 1988) and PLA 2 activity in neuron enriched spinal cord cultures (Farooqui et al., 2001). In vivo intercerebroventricular injections of KA were shown to increase total $\mathrm{PLA}_{2}$ and sPLA 2 activity in the rodent brain (Thwin et al., 2003). Increased 
levels of extracellular glutamate following TBI causes overstimulation of glutamate receptors that may result in secondary events such as $\mathrm{SPLA}_{2}$ release, degradation of membrane phospholipids, and accumulation of free fatty acids, leading to neuronal cell death as well as increased levels of eicosanoids and leukotrienes (McIntosh et al., 1998, Park et al., 2004). As suggested above oxidative stress, EAA, and cytokines could induce $\mathrm{sPLA}_{2}$ release and abnormal phospholipid metabolism and may represent a common mechanism involved in traumatic spinal cord and brain injuries.

Ischemia

$\mathrm{sPLA}_{2}$ has been more thoroughly studies in ischemia. Additionally, ischemia is a component of secondary injury after CNS trauma (Tator, 1991, Tator and Fehlings, 1991, Gennarelli, 1993). Posttraumatic ischemia may result in energy failure that initiates a complex series of metabolic events, ultimately causing neuronal death. One such critical metabolic event is the activation of $\mathrm{PLA}_{2}$ which can result in hydrolysis of membrane phospholipids, release of free fatty acids, generation of oxygen free radicals, and formation of eicosanoids (Nakano et al., 1990, Phillis and O'Regan, 2004, Muralikrishna Adibhatla and Hatcher, 2006).

In both experimental models of brain (Yoshida et al., 1983, Abe et al., 1988, Nakano et al., 1990, Narita et al., 2000) and spinal cord ischemia (Halat et al., 1987) significant increases in the level of free fatty acids, indirectly reflecting PLA $\mathrm{A}_{2}$ activity, were found. Significant increases in $\mathrm{SPA}_{2}$ activities were also reported in vivo following brain ischemia (Lauritzen et al., 1994, Yagami et al., 2002b, Adibhatla et al., 2006) and in astrocytes cultured under ischemic conditions such as oxygen and glucose 
deprivation (Lin et al., 2004). Biphasic increased expression of sPLA 2 -IIA is observed in ischemic rat forebrain (Lauritzen et al., 1994). An early increase in sPLA 2 -IIA mRNA occurred at 1-6 h post-ischemia and a late phase of greater induction of sPLA 2 -IIA appeared between 7 and 20 days post-ischemia. Recently, increased expression of sPLA $_{2}$ IIA has been confirmed at both mRNA and protein levels after brain ischemia (Lin et al., 2004, Adibhatla et al., 2006). Cytokines such as TNF- $\alpha$ and IL-1 $\beta$ have been shown to mediate the ischemia induced $\mathrm{PLA}_{2}$ activation and $\mathrm{SLA}_{2}$-IIA expression in transient focal rat cerebral ischemic model (Adibhatla et al., 2006, Adibhatla and Hatcher, 2007). Indoxam, a specific $\mathrm{PLA}_{2}$ inhibitor, was shown to offer protection against the ischemia induced damage (Yagami et al., 2002b). Quinacrine / mepacrine, a non-specific inhibitor of $\mathrm{PLA}_{2}$ activity, also showed sparing of hippocampal neurons (Phillis, 1996) and reduced infarct size following transient focal ischemia (Estevez and Phillis, 1997). In vitro experiments showed that increased $\mathrm{sPLA}_{2}$ activity was associated with ischemiainduced apoptosis (Yagami et al., 2002b). Other studies have shown cPLA $\mathrm{A}_{2}$ increases following ischemia (Owada et al., 1994, Bonventre et al., 1997, Saluja et al., 1997, Stephenson et al., 1999, Tabuchi et al., 2003) and other authors suggest that $\mathrm{CPLA}_{2}$ rather than $\mathrm{sPLA}_{2}$ mediates neuronal death in ischemia (Arai et al., 2001). In summary, ischemia induces $\mathrm{PLA}_{2}$ activation which could result in deleterious effects such as the loss of membrane integrity through excessive phospholipids hydrolysis, formation of eicosanoids, cytotoxic products, ROS, and induction of apoptosis of affected cells (Sapirstein and Bonventre, 2000, Farooqui et al., 2006).

Other Degenerative Diseases 
Beyond neurotrauma, $\mathrm{sLA}_{2}$ has been suggested as a mediator of neurodegenerative disorders such as Alzheimer's disease (AD) (Moses et al., 2006), Multiple Sclerosis (Pinto et al., 2003, Marusic et al., 2005), and Parkinson's disease (Tariq et al., 2001). AD is characterized by an increased deposition of amyloid plaques infiltrated by reactive astrocytes and microglia. Aggregated forms of amyloid $\beta$ (A $\beta$ ) peptides, particularly A $\beta 1-42$, have been shown to elicit cytotoxic effects resulting in neuron cell death (Sun et al., 2004). There is evidence for alterations in phospholipid levels in patients with AD (Farooqui et al., 1997a). In two separate studies, a decrease in $\mathrm{PLA}_{2}$ activity was found in the parietal and temporal cortices (Ross et al., 1998), as well as in the prefrontal cortex of the AD brain (Talbot et al., 2000). Contrary to these studies, immunohistochemical experiments showed increases in both sPLA - IIA (Moses et al.,

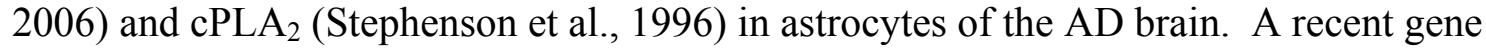
array study in $\mathrm{AD}$ patients indicated an increase in $\mathrm{CPLA}_{2}$ and COX-2 expression, as well as upregulation of a number of apoptotic and proinflammatory genes, but no mention was made of sPLA 2 (Colangelo et al., 2002). These findings are in agreement with the increased oxidative and inflammatory responses and presence of reactive astrocytes associated with AD pathology (Butterfield et al., 2002). In vitro studies demonstrated the ability of $A \beta$ to enhance the activity of a number of phospholipases (Kanfer et al., 1998). Nicotine, a cholinergic agonist, inhibited an $A \beta$-induced increase in $\mathrm{PLA}_{2}$ activation (Singh et al., 1998). The ability of PLA 2 inhibitors to attenuate A $\beta$-induced ROS production could indicate the involvement of $\mathrm{PLA}_{2}$ in $\mathrm{A} \beta$ cytotoxicity (Andersen et al., 2003). For a more thorough review please see (Sun et al., 2004). 
Evidence also linked $\mathrm{SLA}_{2}$ generation to white matter disorders and their experimental equivalents. An early study by Huterer, et al., in the post mortem brains of Multiple Sclerosis patients found no difference in PPLA $_{2}$-IIA activity and a decrease in cPLA $_{2}$ activity within white matter lesions (Huterer et al., 1995). However, more recent studies found that $\mathrm{cPLA}_{2} \alpha-/-$ mice were more resistant to experimental autoimmune encephalomyelitis a rodent model of Multiple Sclerosis. Additionally, $\mathrm{CPLA}_{2} \alpha$ appeared to play a role in both the induction and effecter phases as well as increasing inflammation in the white matter lesions (Marusic et al., 2005). Pinto, et al., found that extracellular inhibitors of $\mathrm{sPLA}_{2}$ were able to decrease CNS inflammation, prevent the induction of proinflammatory cytokines and ameliorate experimental autoimmune encephalomyelitis (Pinto et al., 2003). Finally, in the brains of patients with Krabbe Disease, a demyelinating disease of the CNS, $\mathrm{sPLA}_{2}$ was increased in post mortem human samples, and in twitcher mice, its rodent equivalent. Additionally, the use of a $\mathrm{SPLA}_{2}$ specific inhibitor reduced psychosine-induced oligodendrocyte death in vitro (Giri et al., 2006).

Studies using indirect markers for phospholipid metabolism have also suggested a role for sPLA $\mathrm{P}_{2}$ in Parkinson's disease, a degenerative disease of the CNS characterized by bradykinesia and death of dopaminergic neurons in the substantia nigra (Hayakawa et al., 2001). More importantly, Quinacrine, a nonselective PLA $\mathrm{A}_{2}$ inhibitor, significantly reduced MPTP-induced dopamine loss in an experimental model of Parkinson's disease (Tariq et al., 2001). Mice deficient in cPLA 2 were also shown to exhibit more resistance to MPTP neurotoxicity than wild-type mice, supporting a role for $\mathrm{CPLA}_{2}$ in mediating MPTP neurotoxicity (Klivenyi et al., 1998). 


\section{Conclusions and Direction of Study}

sPLA $_{2}$ is a family of molecules that are closely associated with inflammation.

Secondly, the inflammatory response is thought to exacerbate the damage associated with secondary SCI. Third, inhibition of sPLA $_{2}$ in conditions such as arthritis and ischemic bowel disease lessens the inflammation and resulting pathology associated with these disorders. Current evidence shows that $\mathrm{SPLA}_{2}$ is present in the naive $\mathrm{CNS}$, but it is unknown whether neurotrauma increases its expression. Finally, while the products of $\mathrm{SPLA}_{2}$ metabolism, such as lysophospholipids and AA, are neurotoxic to the CNS and present following SCI and other neuropathologies, it has not been determined whether sPLA 2 's presence directly contributes to the inflammatory state following SCI and if so whether blockade of $\mathrm{sPLA}_{2}$ can create histological and functional improvements.

The focus of this research was to determine if SCI upregulate $\mathrm{SLA}_{2}$ and whether other CNS insults, such as $\mathrm{H}_{2} \mathrm{O}_{2}$ or TNF $\alpha$ and IL-1 $\beta$, can upregulate $\mathrm{SPLA}_{2}$ in cultured oligodendrocytes. Secondly, we sought to determine whether an artificial increase in $\mathrm{SPLA}_{2}$, by exogenous addition of $\mathrm{sPLA}_{2}$, could damage the CNS or cultured cells. Finally, we assessed whether $\mathrm{SPLA}_{2}$ inhibition could produce histological and functional sparing following experimental SCI. 
CHAPTER II

\section{EXPRESSION OF SPLA 2 FOLLOWING SPINAL CORD INJURY}

\section{INTRODUCTION}

Our lab previously showed that total PLA $_{2}$ enzymatic activity in spinal cord homogenates increases after injury, peaking at $4 \mathrm{hr}$ post injury. In the same study we showed that $\mathrm{cPLA}_{2}-\mathrm{IV} \alpha\left(\mathrm{cPLA}_{2} \alpha\right)$ protein expression, while increased following SCI, did not peaked until 7 days post injury (Liu et al., 2006). This discrepancy led us to believe that other PLA 2 isozymes constituted the majority of total phospholipase activity observed following SCI. This lead us to investigate the expression profile of the largest group of $\mathrm{PLA}_{2} \mathrm{~S}$, namely sPLA , following SCI.

There are three rationales for investigating $\mathrm{SPLA}_{2}$ 's role in SCI-induced oligodendrocyte death. First, although $\mathrm{SPA}_{2}$ is found in both neurons and oligodendrocytes its role in neuron death is well documented in association with cerebral ischemia (Yagami et al., 2002b, Yagami et al., 2005) while sPLA,'s role in oligodendrocytes has been effectively ignored. Secondly, when $\mathrm{SPLA}_{2}$ was injected into the border zone between the grey and white matter of the ventral horn, we noticed substantially more inflammation within the white matter (Supp. Fig 1). This suggested that $\mathrm{sPLA}_{2}$ may have a more deleterious effect on white matter. Third, many mediators of secondary SCI are both activators of $\mathrm{sPLA}_{2}$ and cytotoxic to oligodendrocytes. For 
example, hydrogen peroxide injury triggers phospholipid metabolism and AA release in various cell types (Meyer et al., 1996, Tournier et al., 1997, Cane et al., 1998) and $\mathrm{H}_{2} \mathrm{O}_{2}$

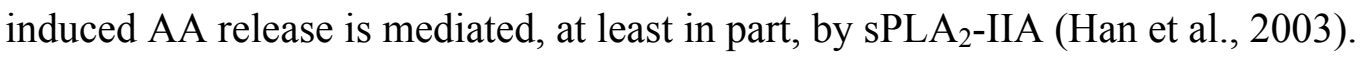
Likewise, IL-1 $\beta$ and TNF $\alpha$ trigger AA release from cultured cells via a sPLA 2 -IIA and cPLA 2 -IV $\alpha$ dependent mechanism (Mounier et al., 2004, Kuwata et al., 2005). Finally $\mathrm{H}_{2} \mathrm{O}_{2}$ (Richter-Landsberg and Vollgraf, 1998, Mronga et al., 2004), IL-1 $\beta$ (Takahashi et al., 2003), TNF $\alpha$ (Selmaj and Raine, 1988, Lee et al., 2000, Buntinx et al., 2004), and AA (Wang et al., 2004) have all been shown to damage cultured oligodendrocytes. Until now, the expression of $\mathrm{SPLA}_{2}$ isoforms after $\mathrm{SCI}$ and their possible role in oligodendrocyte death has not been directly studied. Here we provide cellular and molecular evidence that $\mathrm{SLA}_{2}$-IIA and IIE are the two major $\mathrm{SPLA}_{2}$ isoforms that increase expression hours following SCI and that both isoforms are present within oligodendrocytes. Moreover, administration of $\mathrm{sPLA}_{2}$-IIA in vitro can induce oligodendrocyte cell death. Finally, blockade of $\mathrm{sPLA}_{2}$ can partially ameliorate culture oligodendrocyte cell death induced by either $\mathrm{H}_{2} \mathrm{O}_{2}$ or IL-1 $\beta$ and TNF $\alpha$ injury. Thus, sPLA $_{2}$-IIA may serve as a mediator of oligodendrocyte death and a target for therapeutic intervention against injury-induced oligodendrocyte cell death following SCI.

\section{MATERIALS AND METHODS}

Animals

A total of 82, adult female Sprague-Dawley rats (Harlan, Indianapolis, IN), weighing 200 to $220 \mathrm{gm}$, were used in this study (Table 1). All surgical interventions and postoperative animal care were performed in accordance with the Guide for the Care and 
Use of Laboratory Animals (National Research Council, National Academy Press, Washington, D.C., 1996) and the Guidelines of the University of Louisville Institutional Animal Care and Use Committee.

Table 1. Animal Usage and Experimental Groups

\begin{tabular}{ccccccc}
\hline & Sham & 15 min & $1 \mathrm{hr}$ & $4 \mathrm{hr}$ & 1 day & 1 week \\
\hline Contusion & - & + & + & + & + & + \\
\hline mRNA & $\mathrm{n}=3$ & $\mathrm{n}=3$ & $\mathrm{n}=3$ & $\mathrm{n}=3$ & $\mathrm{n}=3$ & $\mathrm{n}=3$ \\
Protein & $\mathrm{n}=4$ & $\mathrm{n}=4$ & - & $\mathrm{n}=4$ & $\mathrm{n}=4$ & $\mathrm{n}=4$ \\
Subcellular Protein & $\mathrm{n}=4$ & - & - & $\mathrm{n}=4$ & - & - \\
Histology & $\mathrm{n}=5$ & $\mathrm{n}=5$ & - & $\mathrm{n}=5$ & $\mathrm{n}=5$ & $\mathrm{n}=5$ \\
\hline
\end{tabular}

\section{Spinal Cord Injury}

Rats received either a $200 \mathrm{kDyn}$ injury (measured force $=210 \pm 7 \mathrm{kDyn}, \mathrm{C}_{\mathrm{V}}=$ $3.28 \%$ ) between the $4^{\text {th }}$ and $5^{\text {th }}$ cervical vertebra level (C4-5), inflicted via an Infinite Horizons (IH) impactor (Scheff et al., 2003), or sham laminectomy according to a previously published work (Iannotti et al., 2004). Briefly, rats were anesthetized with Nembutal (50 mg/kg, i.p., Abbott Laboratories, Chicago, IL) and a dorsal laminectomy was performed at the C4-5 level to expose the spinal cord. The exposed vertebral column was stabilized using a rat stabilizer developed at the University of Louisville. After the injury, displacements were recorded and compared to insure lesion uniformity $(1086 \pm 93$ 
$\mu \mathrm{m} ; \mathrm{C}_{\mathrm{V}}=8.56 \%$ ). Post-operative care followed those described previously (Iannotti et al., 2004).

RNA Extraction and RT-PCR

A $1.5 \mathrm{~cm}$ long spinal cord segment containing the injury epicenter or equivalent was removed $4 \mathrm{hr}$ post injury, frozen in liquid nitrogen, and later homogenized in STAT60 solution (TelTest, Friendswood, TX). This kit was subsequently used according to the manufacturer's instructions to purify RNA, which was quantified by spectrophotometric analysis of absorbance at $260 \mathrm{~nm}$. Primers used for end point RT-PCR are listed in Table 2. 
Table 2. End point RT-PCR primers.

\begin{tabular}{|c|c|c|c|c|}
\hline Gene & Accession No. & Primer sequence 5'-3' & Product (bp) & Reference \\
\hline $\mathrm{PLA}_{2}$-IB & NM_031585 & $\begin{array}{l}\text { 232-251: ACA ATC AGG CCA AGA AGC TG } \\
\text { 462-481: ACG GCA TAG ACA GGA AGT GG }\end{array}$ & 250 & (Kolko, 2004) \\
\hline $\mathrm{PLA}_{2}$-IIA & NM_031598 & $\begin{array}{l}\text { 687-707: TTGCCATTGTGGTGTGGGTGG } \\
\text { 965-986: CAACTGGGCGTCTTCCCTTTGC }\end{array}$ & 300 & (Molloy, 1998) \\
\hline $\mathrm{PLA}_{2}$-IIC & NM_019202 & $\begin{array}{l}\text { 1-20: CCTCCACCTCTCAAATGCTG } \\
\text { 231-250: CATTGCTGTTCCAGCCTTTT }\end{array}$ & 250 & (Molloy, 1998) \\
\hline $\mathrm{PLA}_{2}$-IID & NM_001013428 & $\begin{array}{l}\text { 1-20: CTGCCTTGCTCTGTGCTGGA } \\
\text { 234-253: CCATCGATCTTCAGGTGGGC }\end{array}$ & 254 & \\
\hline $\mathrm{PLA}_{2}$-IIE & XM_238421 & $\begin{array}{l}\text { 401-419: GTGGGAACCTGGTCCAGTT } \\
\text { 667-687: GGCAGCTCTCTTGTCACACTC }\end{array}$ & 285 & (Kolko, 2004) \\
\hline $\mathrm{PLA}_{2}$-IIF & XM_233589 & $\begin{array}{l}\text { 1-20: ATGAAGGAGGTTGAGTTTGC } \\
\text { 242-261: TGGAATATCACAGAGCTGGA }\end{array}$ & 262 & \\
\hline $\mathrm{PLA}_{2}$-III & XM_223553 & $\begin{array}{l}\text { 12-36: TATACTTGAGTATAAGACCTCGTGT } \\
\text { 243-262: TCAGAAGAATTGAGCAGGAC }\end{array}$ & 251 & \\
\hline $\mathrm{PLA}_{2}-\mathrm{V}$ & NM_017174 & $\begin{array}{l}\text { 380-401: CCCTAAGGATGGCACTGATTGG } \\
\text { 530-551: CCCTAAGGATGGCACTGATTGG }\end{array}$ & 172 & (Molloy, 1998) \\
\hline $\mathrm{PLA}_{2}-\mathrm{X}$ & NM_017176 & $\begin{array}{l}\text { 461-481: TCC CCT CGG TTT TAT GTG AG } \\
\text { 640-660: GCT CCA CAG GCT CAT AGT CC }\end{array}$ & 200 & (Kolko, 2004) \\
\hline $\mathrm{PLA}_{2}$-XIIa & XM_342340 & $\begin{array}{l}\text { 144-163: CCAGGAACAGGACCAGACCA } \\
\text { 373-393: CTTGGTCAGGGAAGGGATGC }\end{array}$ & 250 & \\
\hline
\end{tabular}

Primers for sPLA 2 -IID, IIF, and XIIA were designed using Primer Express 2.0 (Applied Biosystems, Foster City, CA). Each primer set was validitated in mRNA extracted from the liver, lung, kidney, and spleen. This led to a band in the correct weight for at least one of sources for each primer set tested. Total RNA $(0.5 \mu \mathrm{g})$ was used in a $20 \mu \mathrm{l}$ mixture containing $4 \mu \mathrm{l}$ of $5 \mathrm{x}$ reaction buffer, $0.2 \mathrm{mM} \mathrm{dNTP}, 1 \mu \mathrm{M}$ of up and down stream primer, $1 \mathrm{mM}$ MgSO4, $0.1 \mathrm{u} / \mu 1$ AMV Reverse Transcriptase, $0.1 \mathrm{u} / \mu 1 \mathrm{Tfl}$ DNA Polymerase. The reverse transcription was conducted on an Eppendorf Master 
Cycler (Westbury, NY) with a 45 min first strand cDNA synthesis $\left(45^{\circ} \mathrm{C}\right), 2$ min denaturation $\left(94{ }^{\circ} \mathrm{C}\right)$, followed by 35 cycles of synthesis and amplification consisting of 30 seconds $\left(94^{\circ} \mathrm{C}\right), 1$ minute $\left(60^{\circ} \mathrm{C}\right)$, and 2 minutes $\left(72^{\circ} \mathrm{C}\right)($ Access RT-PCR system, Promega, Madison, WI). Amplified samples were separated on a 1\% agarose gel containing ethidium bromide in 1x TBE buffer. After electrophoresis, gels were imaged using an Image Station 4000R (Kodak, Rochester, NY). Finally, each image was quantified using Image J software (NIH, Bethesda MD).

Real-Time Quantitative PCR

Total RNA was extracted from spinal cords, as described above, $4 \mathrm{hr}$ after sham operation or $15 \mathrm{~min}, 1 \mathrm{hr}, 4 \mathrm{hr}, 1$ day, or 1 week after SCI. Primers and taqman probes for sPLA 2 -IB and IIA were designed using Primer Express 2.0 (Applied Biosystems, Foster City, CA) and are as follows: IB sense 5'- AACTACGGCTGCTACTGTGG-3', IB antisense 5'-AGCAGTGGTCATGAGTCTGG-3', IB probe 5'CGGCTCAGGCACCCCAGTGGAC-3', IIA sense 5'CCAAATCTCCTGCTCTACAAACC-3', IIA antisense 5'CTTTTCTTGTTCCGGGCAAAAC-3', and IIA probe 5'CGGCAGCTTTATCGCACTGGCACA-3'. PCR products were detected by measuring the increase in fluorescence on a MX3000P (Stratagene, La Jolla, CA). The sequencedetector software calculated the threshold cycle number $(\mathrm{Ct})$ when signals reached tenfold the standard deviation of the baseline. Primer pairs were chosen to minimize primer dimerization and secondary structure; and to generate an amplicon of $96 \mathrm{bp}$. After PCR, reaction products were electrophoresed on a $1 \%$ agarose gel containing ethidium bromide 
in 1x TBE buffer and then visualized. This step was included to ensure that the only PCR product was at the desired amplicon size. To correct for possible volume differences, transparency of the caps, or other well-to-well differences, the passive reference dye 5(6)carboxy-X-rhodamine-C5-maleimide (also known as ROX; Stratagene) was used in all reactions. Relative expression of the PCR products was determined by using the $\Delta \Delta \mathrm{Ct}$ method (Gibson et al., 1996; Winer et al., 1999). This method calculates relative expression with the equation: - Fold induction $=2[\Delta \Delta \mathrm{Ct}]$, where $\mathrm{Ct}=$ the threshold cycle, i.e., the cycle number at which the sample's relative fluorescence rises above the background fluorescence; and $\Delta \Delta \mathrm{Ct}=[\mathrm{Ct}$ gene of interest (unknown sample) $-\mathrm{Ct} \beta$ actin (housekeeping gene)]. Each sample was run in duplicate, and the mean Ct was used in the $\Delta \Delta \mathrm{Ct}$ equation. After a 3 min denaturation step, the PCRs were subjected to 40 cycles of $30 \mathrm{sec}$ annealing and $30 \mathrm{sec}$ extension at $72{ }^{\circ} \mathrm{C}$.

\section{Western Blotting}

Western blotting followed a previously described procedure (Liu et al., 2004b). In brief, whole cell lyses proteins were extracted from a $1.5 \mathrm{~cm}$ long spinal cord segment containing the injury epicenter $4 \mathrm{hr}$ after sham laminectomy or $15 \mathrm{~min}, 4 \mathrm{hr}, 1$ day or 1 week after SCI. Additionally, subcellular protein isolation was performed on either sham animals or $4 \mathrm{hr}$ after injury using the Focus SubCell kit (G-Biosciences, St. Louis, MO) according to manufacturer's protocol. In brief, $100 \mathrm{mg}$ of fresh tissue was homogenized and centrifuge at $700 \mathrm{xg}$ for 5 minutes to pelletize nuclei. Supernatant was removed and centrifuged at $12,000 \mathrm{x}$ g for $10 \mathrm{~min}$ to pelletize mitochondria. Supernatant was again removed and centrifuged at $14,000 \mathrm{x} g$ for $60 \mathrm{~min}$ to separate the enriched cytosolic 
membrane fraction from the soluble cytosol fraction. The protein content of all samples was assessed by the Bradford method (Bio-Rad Protein Assay, Hercules, CA) and normalized prior to adding sample buffer.

Similar amounts of protein $(40 \mu \mathrm{g})$ was electrophoresed on a $12 \%$ sodium dodecyl sulfate-polyacrylamide gel, transferred onto a polyvinylidene diflouride membrane, and immunoblotted with primary rabbit monoclonal anti-sPLA 2 -I antibody (1:100; “Anti-PLA2, low molecular weight” Millipore, Billerica, MA), polyclonal antisPLA 2 -IIA antibody (1:1000; Cayman Chemical, Ann Arbor, MI), polyclonal antisPLA $_{2}$-IIE antibody (1:1000; Biovendor, Candler, NC), or goat polyclonal anti-sPLA $2-\mathrm{X}$ antibody (1:200; Santa Cruz Biotechnology, Santa Cruz, CA) and a secondary horseradish peroxidase-conjugated donkey anti-rabbit or anti-goat IgG antibody (1:10,000; Amersham Pharmacia Biotech, Piscataway, NJ). The blot was visualized using the ECL Plus Detection Kit (Amersham Pharmacia Biotech). Whole cell lysis membranes were stripped and reblotted with $\beta$-tubulin, to confirm consistent well loading (Liu and $\mathrm{Xu}, 2006)$. Subcellular fraction membranes were stripped and reblotted with $\beta$-tubulin, caveolin, or histone 1 for the cytosol, membrane, and nuclear fractions. The primary antibody was omitted for negative controls. Protein extracted from the rat spleen was used as a positive control for the expression of sPLA 2 -IIA. Densitometry allowed for relative comparison of signal strength by Image $J$ software. When a doublet appeared at the correct weight both bands were included in the analysis.

Immunohistochemistry 
Spinal cords were removed $4 \mathrm{hrs}$ after sham operation or $15 \mathrm{~min}, 1 \mathrm{hr}$, or $4 \mathrm{hr}$ after SCI. After perfusion with PBS and 4\% paraformaldehyde, a $2 \mathrm{~cm}-$ long spinal cord segment containing the injury epicenter of each rat was removed, cryoprotected in $30 \%$ sucrose buffer, sectioned transversely at $40 \mu \mathrm{m}$ on a cryostat, and mounted on Superfrost/Plus slides (Fisher Scientific, Pittsburgh, PA) in eight identical sets. Three sections were taken from each of five sample sites within the tissue, every $0.5 \mathrm{~cm}$, and were incubated with either rabbit monoclonal anti-sPLA 2 -IB (1:200, Chemicon) or rabbit polyclonal anti-sPLA 2 -IIA $(1: 300$, Cayman Chemical $)$ overnight at $4{ }^{\circ} \mathrm{C}$. On the second day, the sections were incubated with secondary biotinylated IgG antibody (1:400; Vector Laboratories, Burlingame, CA) for $1 \mathrm{hr}$ at room temperature. The reaction product was shown by incubation for 5 minutes with $0.02 \%$ diaminobenzidine tetrahydrochloride (DAB) and $0.003 \% \mathrm{H}_{2} \mathrm{O}_{2}$ in $0.05 \mathrm{M}$ Tris- $\mathrm{HCl}(\mathrm{pH}$ 7.6). Slides were examined using an Olympus BX60 light microscope (Olympus America, Inc., Mellville, NY). To further confirm the specificity of the immunohistochemical labeling, negative controls of pooled antibodies from healthy adult animals (Vector Laboratories) in serum blocking solution were used simultaneously.

Using standard light and aperture settings, images were captured with MicroFire Digital Camera (Optronics, Goleta, California) at $2 \mathrm{x}$ and staining intensities were determined using Image $\mathbf{J}$ software (NIH) by first inverting the image and then measuring the mean intensity. The intensity of the negative control animal was subtracted from the recorded intensities to account for unintentional secondary antibody binding and natural tissue coloration. 
Immunofluorescence Labeling

Immunofluorescence double labeling at the injury epicenter was performed on different tissue sets using previously described methods (Liu et al., 2004b). In brief, a mixture of rabbit monoclonal anti-sPLA 2 -IB (1:100; Chemicon), polyclonal anti-sPLA $2_{2}$ IIA (1:100; Cayman Chemical), or polyclonal anti-sPLA 2 -IIE antibody (1:50; Biovendor, Candler, NC), and mouse anti-CC1 (1;100; Chemicon), anti-glial fibrillary acidic protein (1:300; Sigma), and anti-O4 (1:1; hybridoma), anti-NeuN (1:100; Chemicon, Temecula, CA), and anti-SMI-31 (1:2,000; Sigma, St. Louis, MO) antibodies were used to examine colocalization of $\mathrm{sPLA}_{2}$ in neurons, axons, oligodendrocytes, or astrocytes in vivo or mature oligodendrocytes in vitro respectively. The paired antibody solutions were applied to the sections overnight at $4^{\circ} \mathrm{C}$. The following day, sections were incubated with fluorescein-conjugated goat anti-mouse (1:100; Jackson Immunoresearch, West Grove, PA) and Texas red-conjugated goat anti-rabbit (1:100; Jackson Immunoresearch) antibodies. Negative controls of pooled antibodies from healthy adult animals (Vector Laboratories) and normal serum controls were used to further confirm the specificity of the immunofluorescence double labeling. Images were taken using a Nikon Eclipse 90i confocal microscopy (Nikon Instruments; Melville, NY).

sPLA $_{2}$-IIA signal within oligodendrocytes was quantified using Stereo Investigator software (Microbrightfield,Williston, VT) on an Olympus BX60 microscope (Center Valley, PA) under 100x oil immersion. Under standard exposure times contour tracing was begun around the cells of interest while viewed through an FITC filter. After switching to Texas Red filter, luminescence data was acquired for the cell of interest by closing the contour. Twenty oligodendrocytes were chosen from each of three different 
tissue sections, by systematic random sampling from the ventral funiculus of the lesion epicenter. The averaged intensities were averaged to create mean florescent intensity for oligodendrocytes in each animal ( $\mathrm{n}=5$, per group). Background intensity was gathered in a similar manner from primary antibody omission control section and subtracted from the mean intensity for that given animal. This was done to control for non-specific binding of secondary antibody following contusion.

Oligodendroglia Culture

Adult oligodendrocyte precursor cells (aOPCs) were immunopanned with an antiO4 antibody from the adult spinal cord of rats (Kisseberth et al., 1999), using a protocol modified from (Cao et al., 2002). Briefly, the dissected spinal cords were minced into 1 $\mathrm{mm}^{3}$ pieces and incubated in HBSS containing $0.1 \%$ papain, $0.1 \%$ neutral protease, and $0.01 \%$ DNase for $30 \mathrm{~min}$ at $37^{\circ} \mathrm{C}$. The digestion was stopped by the addition of an equal volume of DMEM containing 10\% fetal bovine serum. Tissues were dissociated by repeated trituration with fire-polished Pasteur pipettes and were filtered through a $70 \mu \mathrm{m}$ nylon mesh. The cells were then incubated on an anti-RAN-2 antibody (ATCC, Rockville, MA) coated dish for 30 min to deplete type-1 astrocytes and meningeal cells and then transferred to an anti-O4 antibody-coated dish for $45 \mathrm{~min}$ to select for adult OPCs.

The purified adult OPCs on the dish were removed with $0.05 \%$ trypsin and cultured in DMEM/F12 medium containing N2 and B27 supplements, FGF2 (20 ng/ml), PDGF-aa $(10 \mathrm{ng} / \mathrm{ml})$, Insulin $(5 \mu \mathrm{g} / \mathrm{ml})$ and BSA (0.1\%). An aliquot of cells was analyzed the next day to determine the efficiency of the immunopanning. Only those cell 
preparations in which $>95 \%$ of the bound cells expressed A2B 5 were used in the experiments. The results were confirmed by FACS analysis.

The cells were seeded onto either PDL / laminin coated culture dishes for survival experiments and western blot analysis, or chamber slides for immunocytochemistry. Two days after seeding or once cells reached $70-80 \%$ confluence, the FGF-2 and PDGF-aa were removed, and CNTF $(0.001 \mu \mathrm{g} / \mathrm{ml})$ was added to the OPC medium to induce differentiation. $\mathrm{A} 2 \mathrm{~B} 5^{+} \mathrm{OPCs}$ were induced to differentiate in vitro for 4 days and were either challenged to induce injury, lysed for western blot, or fixed for immunocytochemical analysis.

Mature oligodendrocyte cultures were injured with recombinant human sPLA2-IIA (Biovendor, Candler, NC) and assayed at $48 \mathrm{hr}$ for cytotoxicity by lactate dehydrogenase (LDH) present in the medium (CytoTox96 non-radioactive assay; Promega, Madison, WI) or reduction of (3-(4,5-Dimethylthiazol-2-yl)-2,5-diphenyltetrazolium bromide by the mitochondria of surviving cells (MTT, CellTiter 96 Cell Proliferation Assay; Promega, Madison, WI).

A second set of cultures were pretreated with vehicle, or $0.25,1.25,6.25 \mu \mathrm{M}$ of S3319, a sPLA2-IIA inhibitor diluted in 1\% DMSO (Sigma Aldrich, St. Louise, MO) or $50 \mu \mathrm{M}$ of 4-aminophenyl $\alpha$-D-mannopyranoside, non-catalytic sPLA $\mathrm{A}_{2}$ receptor ligand (APMP, Sigma Aldrich, St. Louise, MO). Then cells were challenged with vehicle, $1 \mathrm{mM}, 5 \mathrm{mM}$, or $10 \mathrm{mM} \mathrm{H}_{2} \mathrm{O}_{2}$, with or without sPLA 2 -IIA inhibitor for $30 \mathrm{~min}$, washed once with fresh medium, and the vehicle or S3319 was replaced. Again cytotoxicity was evaluated by measuring LDH released in the media $48 \mathrm{hr}$ after $\mathrm{H}_{2} \mathrm{O}_{2}$ exposure. 
A third set of cultures were pretreated as above with S3319 but were challenged with the cytokines IL-1 $\beta$ (PeproTech Inc., Rocky Hill, NJ) and TNF $\alpha$ (PeproTech) at low (1 and $2 \mathrm{ng}$ respectively) or high dose ( 5 and $10 \mathrm{ng}$ ). Again cytotoxicity was evaluated by measuring LDH released in the media. Data were normalized to the amount of LDH released from similarly-treated cells receiving a lysis buffer and are corrected for baseline LDH release from blank wells. This procedure was necessary to ensure that the reported effects are not attributable to the various vehicles used or cell death due to pretreatment with S3319. All cell culture experiments consisted of at least 4 separate wells. All experiments were repeated in triplicate on separate days. The results presented are the averaged of the three separate experiments. The area of process extensions was calculated using three separate images take from the center of each well. Images were converted to a binary by setting a common constant threshold and then the area was determined by Image $\mathrm{J}$ software.

Statistical Analysis

A Student's t-test, two tailed, was used to compare RT-PCR results utilizing Levene's Test for Equality of Variances. One-way analysis of variance (ANOVA) with post hoc Tukey HSD was used to determine statistical significance of three or more groups. A multiple analysis of variance (MANOVA) with post hoc Tukey's HSD was used to determine statistical significance of three or more groups when repeated measures were taken from each animal overtime or space. A $p$ value less than 0.05 was considered statistically significant. Two extreme outliers were excluded from the Q-PCR data using the Grubb's extreme studentized deviate method. 


\section{RESULTS}

sPLA $_{2}$ mRNAs are differentially expressed following SCI.

End point RT-PCR was used to scan for the expression of all the sequenced mammalian sPLA 2 isoforms in either sham or contused animals $4 \mathrm{hr}$ following surgery (n = 3). Primer sets were designed for sPLA 2 -IB, IIA, IIC, IID, IIE, IIF, V, X, and XIIA. Seven of the nine mammalian $\mathrm{sPLA}_{2}$ were detected in naïve and contused animals with mRNA present for sPLA 2 -IB, IIA, IIC, IIE, V, X, and XIIA (Fig. 5). Pictured are three representative animals for each isoform that yielded replication. 
Figure 5. Changes in secretory $\mathrm{PLA}_{2}$ mRNA following SCI.

A) Gels shown are representative samples of mRNA levels from either 3 sham-operated rats (lanes 2-4) or 3 rats sacrificed at $4 \mathrm{hr}$ post-SCI (lanes 5-7). Lane 1 is a $100 \mathrm{bp}$ ladder. Total RNA samples were reverse transcribed for all the mammalian $\mathrm{SPLA}_{2}$ isoforms. Statistical increases were seen in $\mathrm{SPLA}_{2}$-IIA and IIE with a decrease seen in $\mathrm{SPLA}_{2}-\mathrm{X}$. No change was observed in $\mathrm{SPLA}_{2}-\mathrm{IB}, \mathrm{IIC}, \mathrm{V}$, and XIIA. B) Relative fold increase of sPLA $_{2}$-IIA mRNA expression after real time Q-PCR at $15 \mathrm{~min}, 1 \mathrm{hr}, 4 \mathrm{hr}, 1$ day, or $1 \mathrm{wk}$ following $\mathrm{SCI}$ (mean $\pm \mathrm{SD})$. 

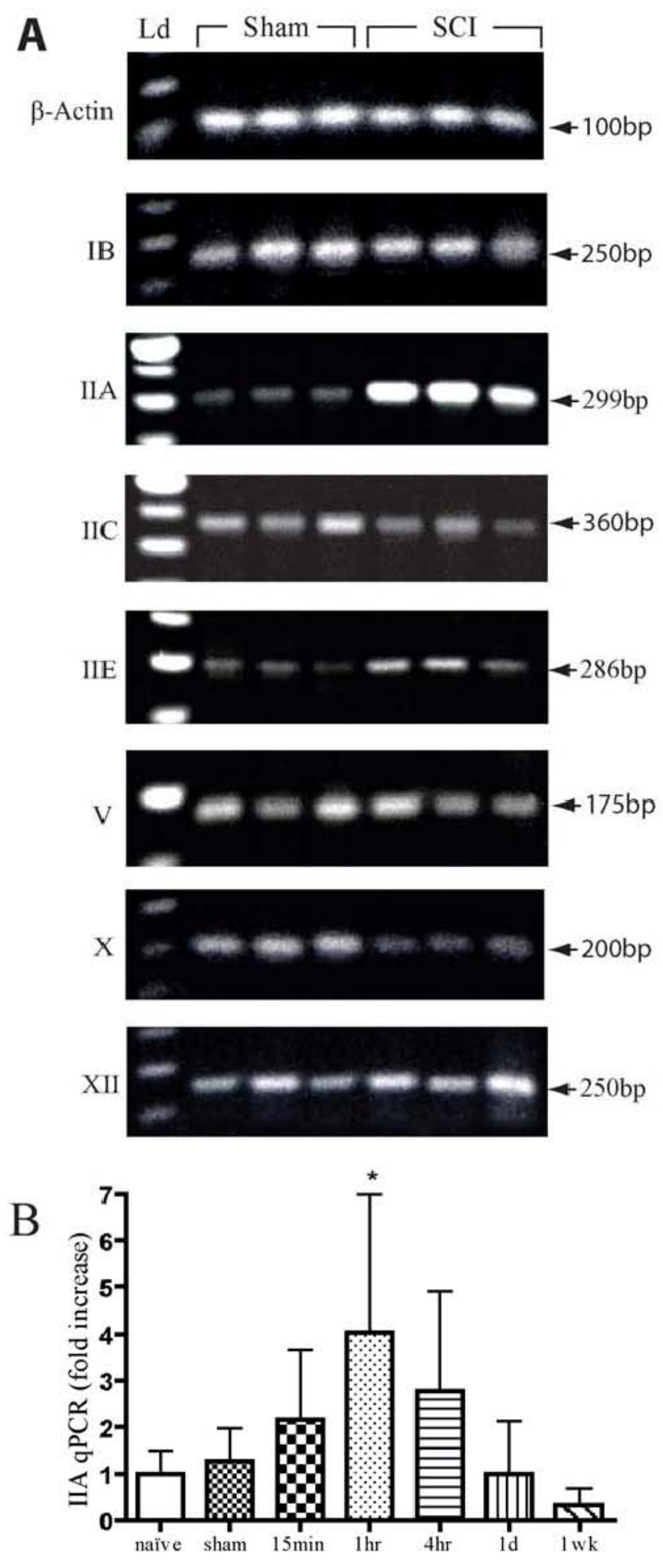
Among all sPLA $\mathrm{A}_{2}$ isoforms examined, sPLA $\mathrm{SL}_{2}$-IIA showed the most dramatic

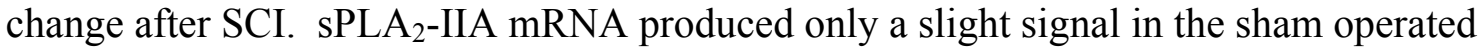
cord but increased $(t(4)=6.44, p=.003$ ) following contusion (Fig. 5A). Similarly, sPLA 2 -IIE, while almost absent in the naïve cord, showed an increase in the injured cord $(t(2.42)=3.93, p=.043)$. In contrast, $\mathrm{PLA}_{2}-\mathrm{X}$ showed a significant decrease in signal intensity $(t(2.53)=5.980, p=.015)($ Fig. $5 \mathrm{~A})$. sPLA 2 -IB, IIC, V and XIIA were present in both the sham and contused cord in similar intensity following RT-PCR. Thus, we demonstrated, for the first time, that $\mathrm{sPLA}_{2}$ isoforms are differentially expressed following a contusive SCI.

Next, real-time Q-PCR was used to quantify the qualitative increases seen after RT-PCR and determine the time course of sPLA ${ }_{2}$ expression following SCI. Two sPLA 2 isoforms, IIA and IB, were chosen for this study because of their significant association with inflammation or highest expression in the CNS, respectively (Kolko et al., 2004). Both subgroups were quantified as a fold increase from naïve animals (Fig. 5B). In agreement with the RT-PCR results, $\mathrm{sPLA}_{2}$-IIA mRNA expression had a significant 4fold increase at $1 \mathrm{hr}$ following contusion compared to the sham operated animal (ANOVA, $\mathrm{F}_{6,30}=2.885, p=0.02$ ). Similar to the end point PCR, sPLA $_{2}$-IB showed no change at $4 \mathrm{hr}$ but by one day a statistically significant 2.54 fold decrease was observed that persisted through one week (ANOVA, $\left.\mathrm{F}_{6,11}=3.649, p=0.04\right)$ (data not shown). This data suggests that isoforms of sPLA $\mathrm{P}_{2}$ are differentially regulated following traumatic $\mathrm{SCI}$ and that $\mathrm{SPLA}_{2}$-IIA synthesis dramatically increases and peaks at $4 \mathrm{hr}$ following contusion. 
${ }_{s P L A}$ proteins are differentially expressed following SCI.

To confirm that changes in mRNA in fact reflect changes in protein production, spinal cord homogenates were immunoblotted with antibodies to sPLA 2 -IB, IIA, IIE, or $\mathrm{X}$. While a general increase in the mean expression of sPLA 2 -IB was seen, this was not statistically significant further confirming the PCR results (Fig. 6A \& B; ANOVA, $\mathrm{F}_{4,15}=$ $0.8335, p=0.52$ ). In contrast, PPLA $_{2}$-IIA showed a 3 -fold increase in protein expression at $4 \mathrm{hr}$ compared to sham controls (Fig. $2 \mathrm{~A} \& \mathrm{C}$, ANOVA, $\mathrm{F}_{4,15}=4.860, p<0.01$ ). Significantly increased sPLA 2 -IIA expression was also found at both $4 \mathrm{hr}$ and 1day post injury (Fig. 6C). An equal amount of splenic protein was run as a positive control for sPLA 2 -IIA and showed a band about 3.6 times greater than the strongest spinal cord band, confirming the lower abundance in the spinal cord as compared to the spleen (Fig. 6A). Not surprisingly, $\mathrm{sPLA}_{2}$-IIE showed similar results to IIA with a significant 2.5 fold-increase that peaked at $4 \mathrm{hr}$ after SCI and returned to the baseline by 1 day (Fig. 6A $\& \mathrm{D}$, ANOVA, $\left.\mathrm{F}_{4,15}=5.025, p<0.01\right)$. Finally, while $\mathrm{sPLA}_{2}-\mathrm{X}$ mRNA decreased following SCI there was little change in the protein levels compared to the sham controls (Fig. 6A \& E, ANOVA, $\mathrm{F}_{4,15}=0.2554, p=0.90$ ). These results confirm that both $\mathrm{sPLA}_{2}-$ IIA and IIE proteins are significantly increased following SCI. Interestingly, the peak expression of the two $\mathrm{SPLA}_{2}$ isoforms coincides well with the peak activation of total $\mathrm{PLA}_{2}$, as we demonstrated previously (Liu et al., 2006). Thus, PLAA $_{2}$-IIA and IIE may represent major contributors to phospholipase activity following SCI. 
Figure 6. Temporal changes in SPLA $A_{2}$ protein expression following SCI.

A) Representative western blots of whole cell lysates from sham or contused animals at $15 \mathrm{~min}, 4 \mathrm{hr}, 1 \mathrm{day}$, or $1 \mathrm{wk}$ post injury. The top panel in A shows a representative time course of sPLA 2 -IB (16 \& $14 \mathrm{kDa})$ protein expression showing no changes following SCI. SCI induced a significant increase in SPLA $_{2}$-IIA $(15 \mathrm{kDa})$ and IIE (18 kDa) expression beginning at $4 \mathrm{hr}$ and continuing through 1 day post injury, but returning to baseline by 1 week. Group X (13 kDa) showed no change in expression. B - E) Quantification of western blots in A from four separate animals per group ( $n=4$ /group, mean $\pm \mathrm{SD})$. $\beta$-tubulin $(50 \mathrm{kDa})$ was used to confirm even sample loading in all blots. $(* * p<0.01$ versus sham) 
A
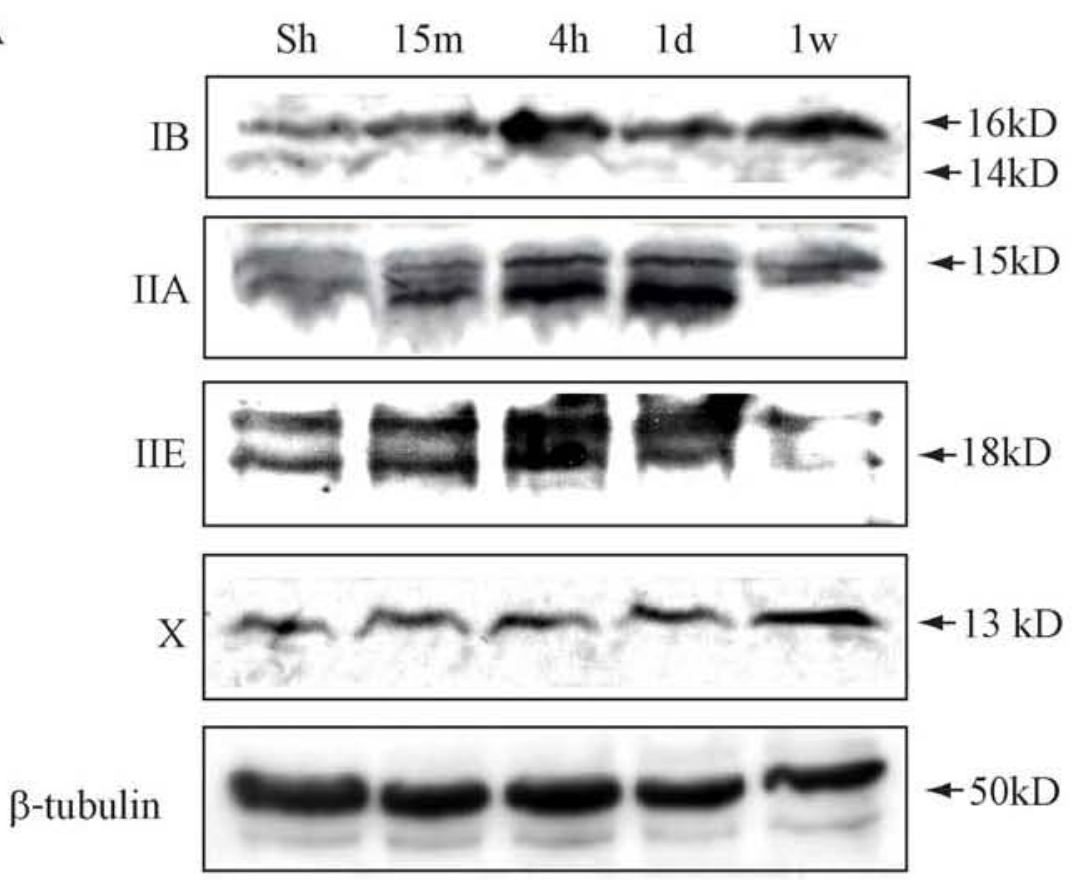

B

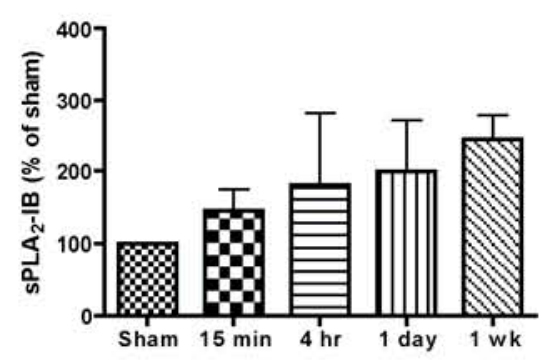

D

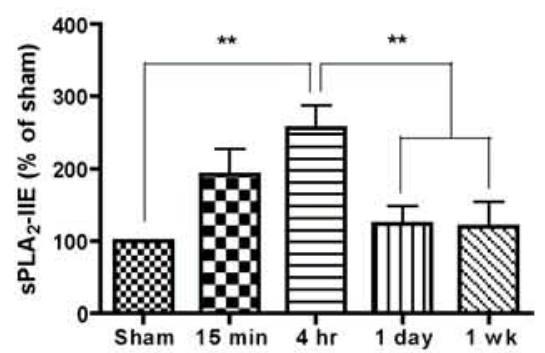

C

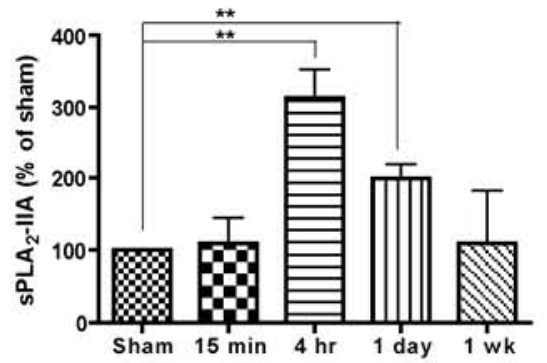

E

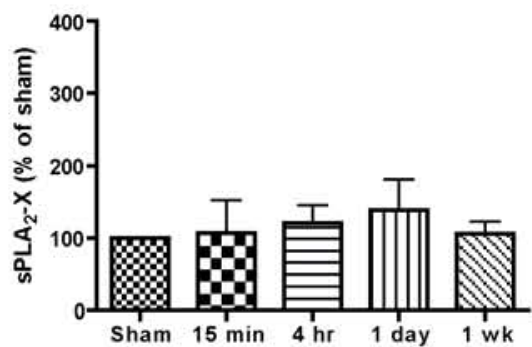


Spatiotemporal distribution of $\mathrm{sPA}_{2}$ isozymes following SCI

Since $\mathrm{SPLA}_{2}$ mRNA and proteins were differentially expressed post SCI, we next determined their temporal and spatial distribution using immunohistochemistry. In this study, sPLA 2 -IB, IIA, IIE, and X were examined at $5 \mathrm{~mm}$ intervals from the injury epicenter.

We chose to focus on white matter because we previously showed that bilateral injections of $\mathrm{sPLA}_{2}$-III $(0.1 \mu \mathrm{g})$ into the ventral grey matter / white matter interface of rat spinal cords resulted in a massive destruction of white matter with a relative sparing of grey matter at 4 weeks (Sup. 1)(Liu et al., 2006). sPLA 2 -IB showed strong expression in white matter (Fig. 7A \& E) both before and after injury. Quantification of labeling intensity using Image $\mathrm{J}$ showed no change in $\mathrm{SPLA}_{2}$-IB signaling either among different time points post $\mathrm{SCI}$ or distance from the epicenter $\left(\mathrm{MANOVA}_{3,263.806}=4.367, p>\right.$ 0.05, Fig. 7I).

In contrast, sPLA 2 -IIA (Fig. 7, $2^{\text {nd }}$ column) and IIE (Fig. 7, $3^{\text {rd }}$ column) each showed a weak baseline staining in the white matter of sham controls. However, following SCI, sPLA2-IIA and IIE immunoreactivity increased markedly (Fig. 7F \& G). Within the white matter, a strong increase in PLA $_{2}$-IIA and IIE staining in glial cells and axons, particularly swollen axons, was seen (Fig. 3F, arrows). Quantification data showed a significant increase in $\mathrm{SPLA}_{2}$-IIA immunoreactivity at the injury epicenter in all SCI groups over sham controls (MANOVA $F_{18.3,15.575}=46.437, p<0.001$, Fig. 7J). Finally, sPLA 2 -X showed little immunoreactivity either in sham or SCI animals (Fig. 7, $4^{\text {th }}$ column). 
Thus, the present study showed that IIA and IIE, but not IB and X, are the major $\mathrm{sPLA}_{2}$ isoforms that markedly increased following SCI and their expression was induced mainly at the site of injury. It should be noted that IIA and IIE are structurally and functionally similar and are both located at the same genetic locus (Kudo and Murakami, 2002). 
Figure 7. Immunohistochemical Changes in sPLA 2 -IB, IIA, IIE, and X Expression Following SCI.

A - H) Changes in the ventral white matter in sham (A - D) or $4 \mathrm{hr}$ post contusion $(\mathrm{E}-\mathrm{H})$. Little change in $\mathrm{SPLA}_{2}$-IB staining was seen (A \& E). In contrast, SCI induced a significant increase in $\operatorname{sPLA}_{2}$-IIA (B \& F) and IIE (C \& G) IR within the white matter. Both IIA and IIE showed staining of axons and glia. However, there was little or no staining of $\mathrm{SPLA}_{2}-\mathrm{X}(\mathrm{D} \& \mathrm{H})$. I \& J) Quantification of IB and IIA staining intensity is shown at the epicenter and at $5 \mathrm{~mm}$ increments, both rostral and caudal to it. Increases above sham controls were only seen in $\mathrm{SPLA}_{2}$-IIA and were only present at the injury epicenter of sPLA 2 -IIA. Bar: A-H, $500 \mu \mathrm{m}$. I-J; $\mathrm{n}=5$ per group, mean $\pm \mathrm{SD} ;{ }^{*} p<0.05$; sham $\rightarrow, 15 \mathrm{~min} \stackrel{\leftarrow}{-}, 1 \mathrm{hr} \rightarrow, 4 \mathrm{hr}^{\bullet}$. 

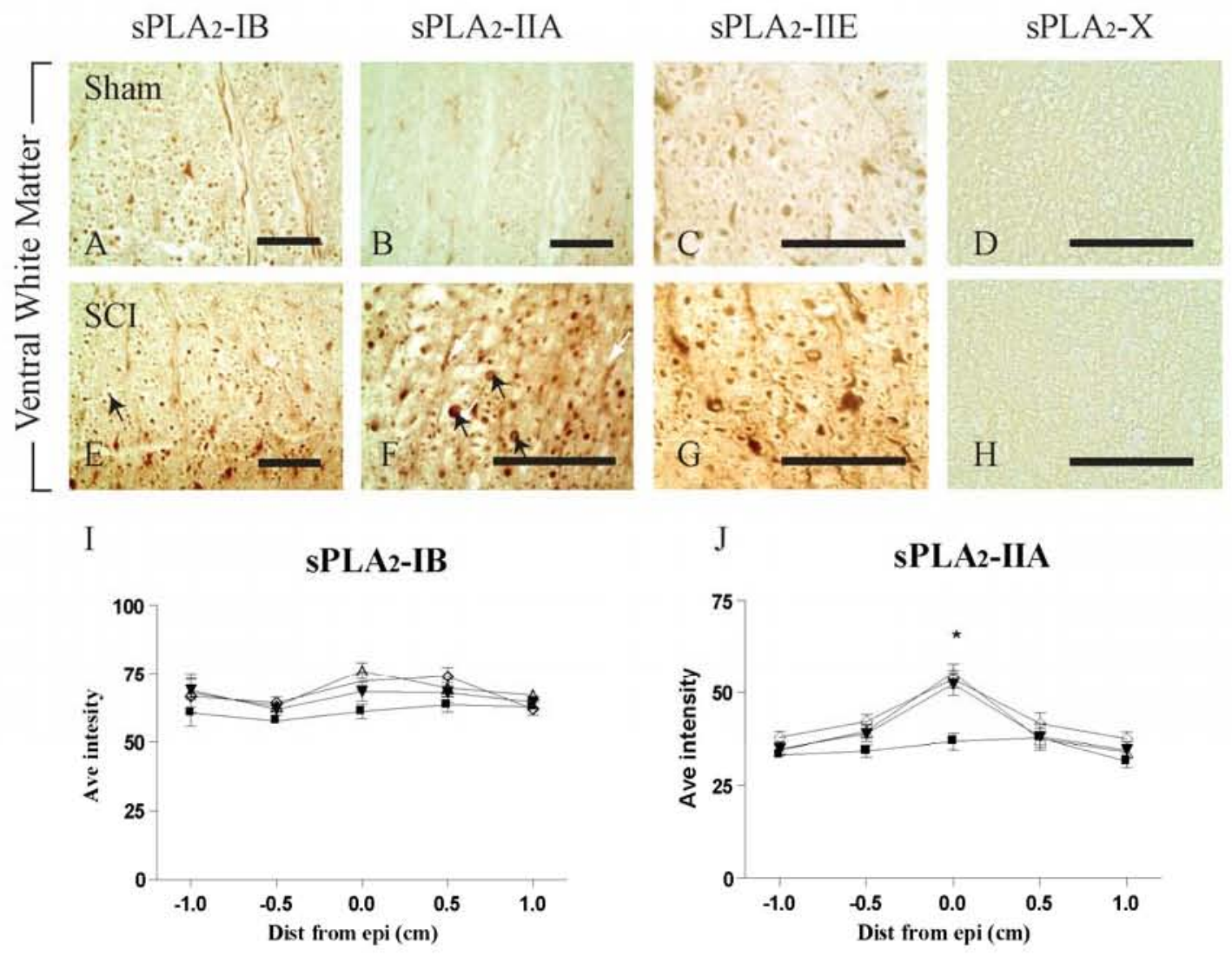
Cellular localization of $\mathrm{PLA}_{2}$ isozymes following SCI

To better understand what cell types express $\mathrm{sPLA}_{2}$ isoforms, immunofluorescent double labeling of sPLA ${ }_{2}-\mathrm{IB}$, IIA, IIE, or $\mathrm{X}$ and cell specific markers was performed. As was suggested by the immunohistochemistry, we found that oligodendrocytes (CC1/APC; Fig. 8) co-localized with sPLA 2 -IB (Fig. 8B \& C), sPLA 2 -IIA (Fig. 8E \& F), and sPLA $2_{2}$ IIE (Fig. 8H \& I). However, sPLA 2 -X did not appear to co-localize with oligodendrocytes (Fig. 8J-L). The sPLA 2 - X staining appeared to reside only in the extracellular space. This morphology is not surprising since $\mathrm{SLA}_{2}-\mathrm{X}$ has the greatest secreted fraction and the lowest cytosolic fraction of any sPLA $\mathrm{S}_{2}$ (Murakami et al., 2002b). Additionally, previous work with stably transfected cell lines yielded similar sPLA 2 -X immunofluorescence (Kudo and Murakami, 2002). 


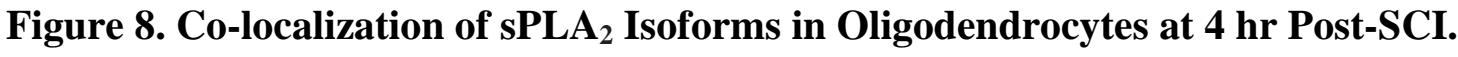
Confocal images of immunofluorescent double labeling indicates that sPLA 2 -IB (A-C), IIA (D-F), IIE (G-I), but not X (J-L) are present within oligodendrocytes (CC1/APC) $4 \mathrm{hr}$ following contusion. All images are orthogonal views of confocal image stacks with the point of interest indicated by the green cross hairs in the merged image. Within each frame the $\mathrm{X} / \mathrm{Y}$ plane is represented by the square picture, the $\mathrm{Y} / \mathrm{Z}$ plane is represented to the right, and the $\mathrm{X} / \mathrm{Z}$ plane is represented along the bottom of the $\mathrm{X} / \mathrm{Y}$ frame. Scale bars: $20 \mu \mathrm{m}$ for all. 

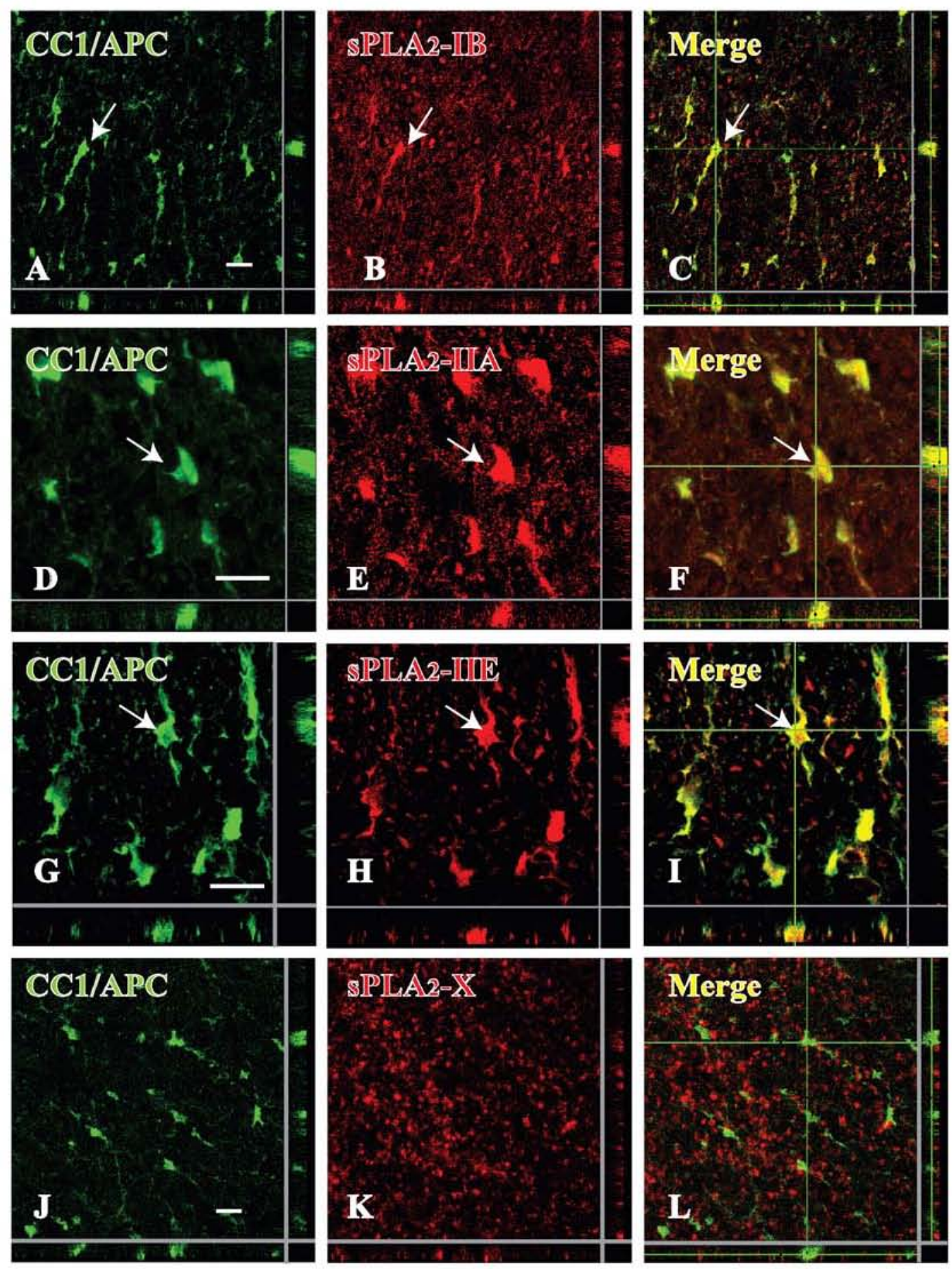
Within the white matter, astrocytes (GFAP; Sup. 2) co-localized with sPLA 2 -IB (Sup. 2B \& C) and weakly with sPLA 2 -IIA (Sup. 2E \& F) but showed no co-localization with sPLA 2 -IIE (Sup. 2H \& I). Similar to staining seen in oligodendrocytes sPLA S $_{2}$ X again did not appear to co-localize with any astrocytes (Sup. 2J-L). Neurons within the ventral grey matter (NeuN; Sup. 3), showed similar staining to oligodendrocytes with the presence of $\mathrm{sPLA}_{2}$-IB (Sup. 3B \& C), sPLA 2 -IIA (Sup. 3E \& F), and sPLA 2 -IIE (Sup. 3H \& I), but not sPLA $2-X($ Sup. 3K \& L). Almost all axons (SMI-31; Sup. 4) within the ventral white matter showed co-localization with $\mathrm{SPLA}_{2}$-IB (Sup. 4B \& C) and $\mathrm{sPLA}_{2}-$ IIA (Sup. 4E \& F). sPLA 2 -IIE staining was weak and confined mainly in axons that appeared to be swollen (Sup. 4H \& I, arrows) and no axons appeared to co-localize with sPLA $_{2}$ X (Sup. 4K \& L).

To further confirm that sPLA 2 -IIA was not merely present in oligodendrocytes but increases following contusion, we compared $\mathrm{sPLA}_{2}$-IIA labeling intensities within oligodendrocytes at the injury epicenter $4 \mathrm{hr}$ after either sham surgery (Fig. 9A-C) or SCI (Fig. 9D-F). A significant increase in $\mathrm{SPLA}_{2}$-IIA expression was found within oligodendrocytes $\left(t(5.947)=-3.517, p=0.01\right.$, Fig. 9G). Increased expression of $\operatorname{sPLA}_{2} \mathrm{~S}$, particularly IIA, opens a new possibility that it may play a role in the death of oligodendrocytes following SCI.

sPLA $_{2}$-IIA increases in membrane fraction following SCI.

To determine whether sPLA, particularly IIA, play a role in increased phospholipid hydrolysis, we determined the co-localization of sPLA 2 with its 
phospholipid substrate, which is most abundant in the membrane fraction of cells. Subcellular fractions of sham and contused spinal cords were immunoblotted for SPLA ${ }_{2}-$ IIA expression. Four hours following contusion, SPLA $_{2}$-IIA increased about 4-fold within the cytosolic membrane fraction (Fig. 9H). While the non-membrane fraction of the cytosol did show a strong band for $\mathrm{PLA}_{2}$-IIA there was little increase following contusion. Additionally, the nuclear fraction showed no sPLA 2 -IIA expression. These results suggest that the increase in $\mathrm{SPLA}_{2}$-IIA protein expression observed in the whole cell lysates after SCI is within the membrane fraction which is rich in phospholipid substrates suggesting a functional role $\mathrm{SPA}_{2}$-IIA on its phospholipid substrates. 
Figure 9. sPLA2-IIA Expression Increases in Oligodendrocytes Following SCI and Increases Within the Membrane Fraction.

A-C) In a sham-operated rat, sPLA 2 -IIA was localized in a CC1-positive oligodendrocyte at a baseline level. D-F) However, at $4 \mathrm{hr}$ post-injury, sPLA $\mathrm{A}_{2}$-IIA expression increased in CC1-positive oligodendrocytes (E versus B). Images were taken under identical conditions within one session. G) Quantification of the sPLA 2 -IIA staining intensity in oligodendrocytes at the injury epicenter $(n=3$ animals per group, mean $\pm \mathrm{SD} ; * *$ $\mathrm{p}<0.01$ ). H) Western blots of subcellular fractions taken from either sham or contused spinal cords $4 \mathrm{hr}$ following injury showing the increase of sPLA 2 -IIA observed in A is predominantly within the membrane bound fraction and not the soluble cytosolic or nuclear fractions. 

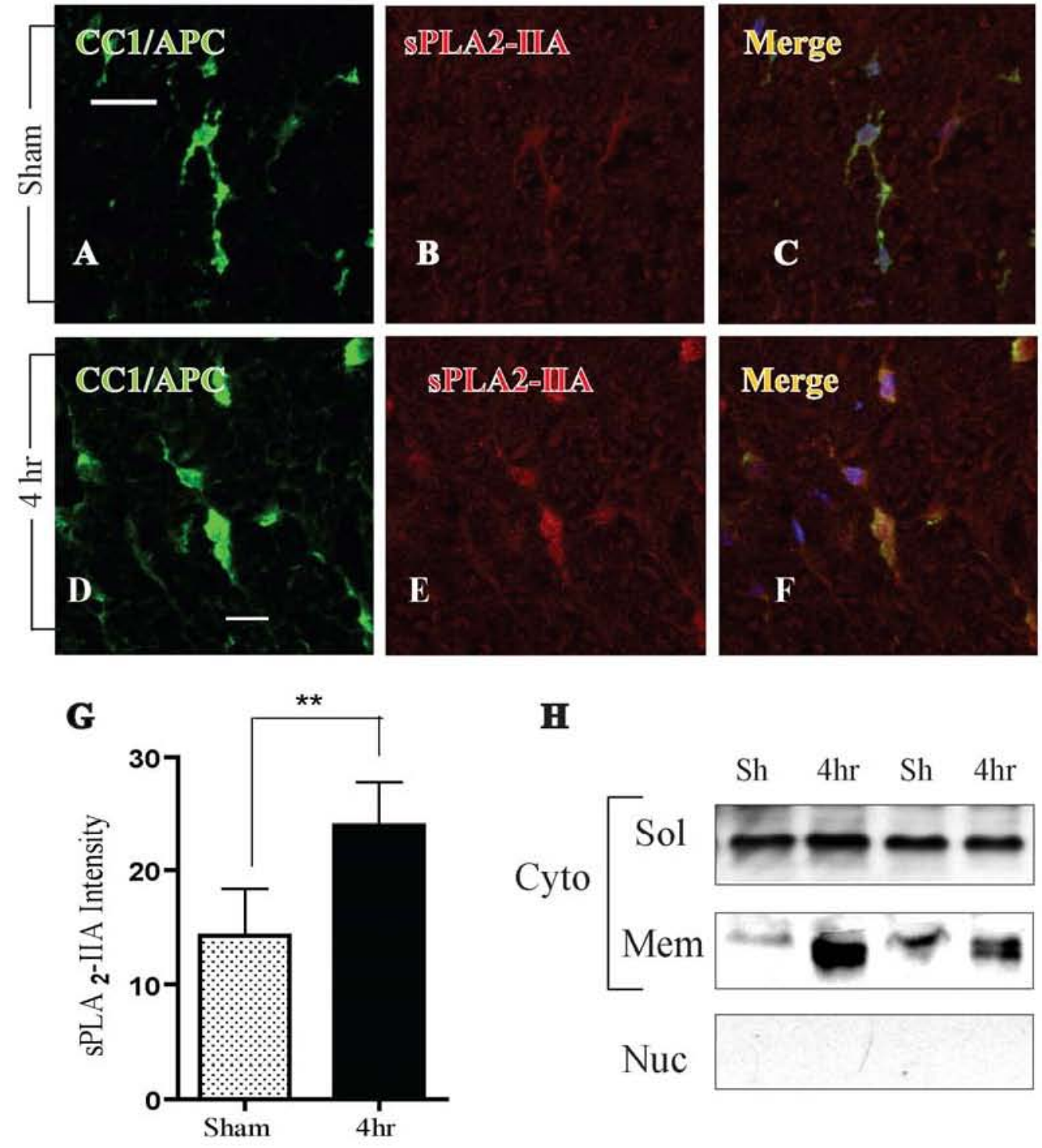

H

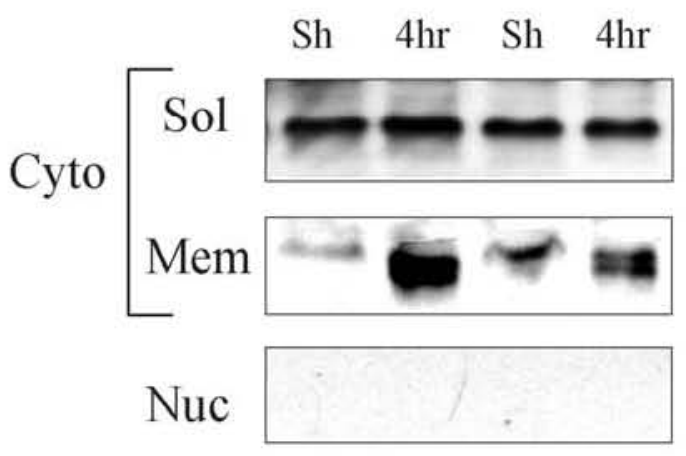


${ }_{\text {PLA }}$-IIA induces oligodendrocyte death in vitro.

Since sPLA $\mathrm{PL}_{2}$-IIA increased globally and within the membrane fraction following SCI, we next examined what effect increased SPLA $_{2}$-IIA might have on oligodendrocytes. These studies focused on oligodendrocytes for two reasons. First, whereas sPLA 2 -IIA induced neuronal apoptosis has already been shown (Yagami et al., 2002b, DeCoster, 2003, Yagami et al., 2003), the role of sPLA2-IIA in oligodendrocyte cell death remains unknown. Secondly, our previous observation indicates that $\mathrm{SPLA}_{2}$ is potentially more destructive to white than grey matter (Sup. 1).

To determine whether sPLA 2 -IIA can directly affect oligodendrocyte viability, recombinant human $\mathrm{sPLA}_{2}$-IIA was added to differentiate adult oligodendrocyte precursor cultures. sPLA $_{2}$-IIA triggered a dose-dependent increase in LDH within the culture medium corresponding to an increase of cell cytotoxicity from $16.0 \pm 5.3 \%$ in control wells to $34.3 \pm 6.3 \%$ in wells treated with $2 \mu \mathrm{M}$ of sPLA $\mathrm{s}_{2}$-IIA at $48 \mathrm{hr}\left(\mathrm{F}_{3,16}=\right.$ 13.04, $\mathrm{p}<0.001$; Fig. 10E). Similarly, the MTT assay showed a corresponding change with control wells showing an optical density (OD) of $0.040 \pm 0.005$ and the $2 \mu \mathrm{M}$ sPLA $_{2}$-IIA dose resulting in an OD of $0.027 \pm 0.005\left(\mathrm{~F}_{3,16}=13.04, \mathrm{p}<0.001\right.$; Fig. 10F $)$. Phase contrast images of these wells confirmed oligodendrocyte damage with a decreased arbor in the 0.01 and $0.1 \mu \mathrm{M}$ concentrations and a complete degradation of the cell soma and processes at the $2 \mu \mathrm{M}$ concentration (Fig. 10A-D). Since sPLA $\mathrm{A}_{2}$-IIA administration

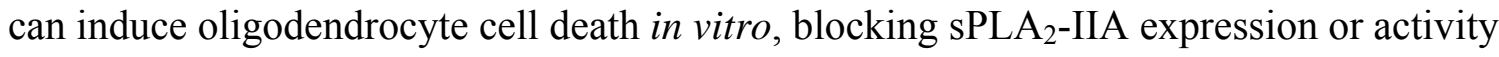
may prevent such cell death following injury.

To determine the relative specificity of sPLA $\mathrm{AL}_{2}$-IIA's effect on oligodendrocytes, identical concentrations were added to primary astrocytes and Schwann cells cultures. 
Interestingly, no cell death was noted in either culture suggesting that sPLA2-IIA has little effect on other glia within the CNS and that the myelinating cells of the peripheral nervous system show resistance to $\mathrm{SPLA}_{2}$ activity. These findings might explain why $\mathrm{sPA}_{2}$ injections into the spinal cord white matter result in oligodendrocyte death but shows strong gliosis and delayed Schwann cell remyelination (Titsworth et al., 2007). Since sPLA $\mathrm{A}_{2}$-IIA administration can induce oligodendrocyte and neuron death in vitro, blocking sPLA $\mathrm{A}_{2}$-IIA expression or activity may prevent such cell death following injury. 


\section{Figure 10. Oligodendrocytes show sensitivity to SPLA $_{2}$-IIA triggered cell death}

relative to Schwann cells and astrocytes.

Low levels of exogenously added sPLA $_{2}$-IIA $(0.01$ and $0.1 \mu \mathrm{M}$; B \& C) result in a loss of processes extending from the soma and a higher dose $(2 \mu \mathrm{M}$; D) triggers loss of process and cell death as shown by phase contrast images. An $18.2 \%$ increase in cytotoxicity as measured by an increase in lactate dehydrogenase within the media (LDH, E), and a $33.1 \%$ decreased conversion of tetrazolium into a formazan product (MTT, F) suggesting decreased cell survival. In contrast the $2 \mu \mathrm{M}$ had no effect on either LDH release or MMT conversion in cultured Schwann cells (F-G) or astrocytes (H-I) suggesting a specific sensitivity to $\mathrm{SPLA}_{2}$-IIA in oligodendrocytes. 

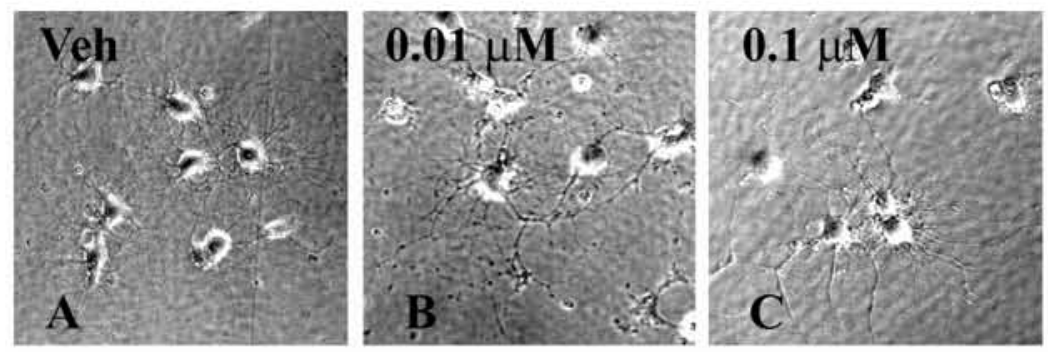

$2 \mu \mathrm{M}$

정
음
$\frac{0}{0}$
$\frac{0}{0}$
음
$\frac{0}{0}$

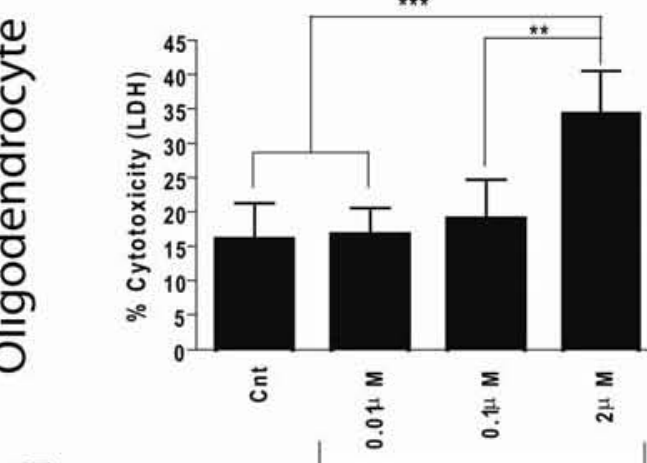

E

sPLA $A_{2}-\| A$

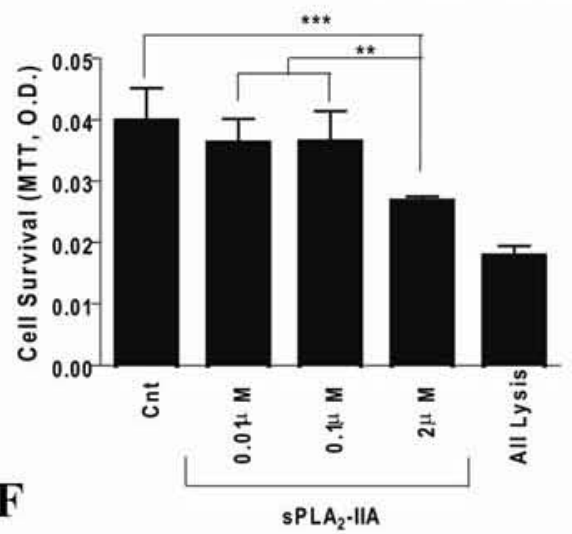

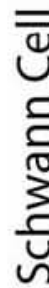
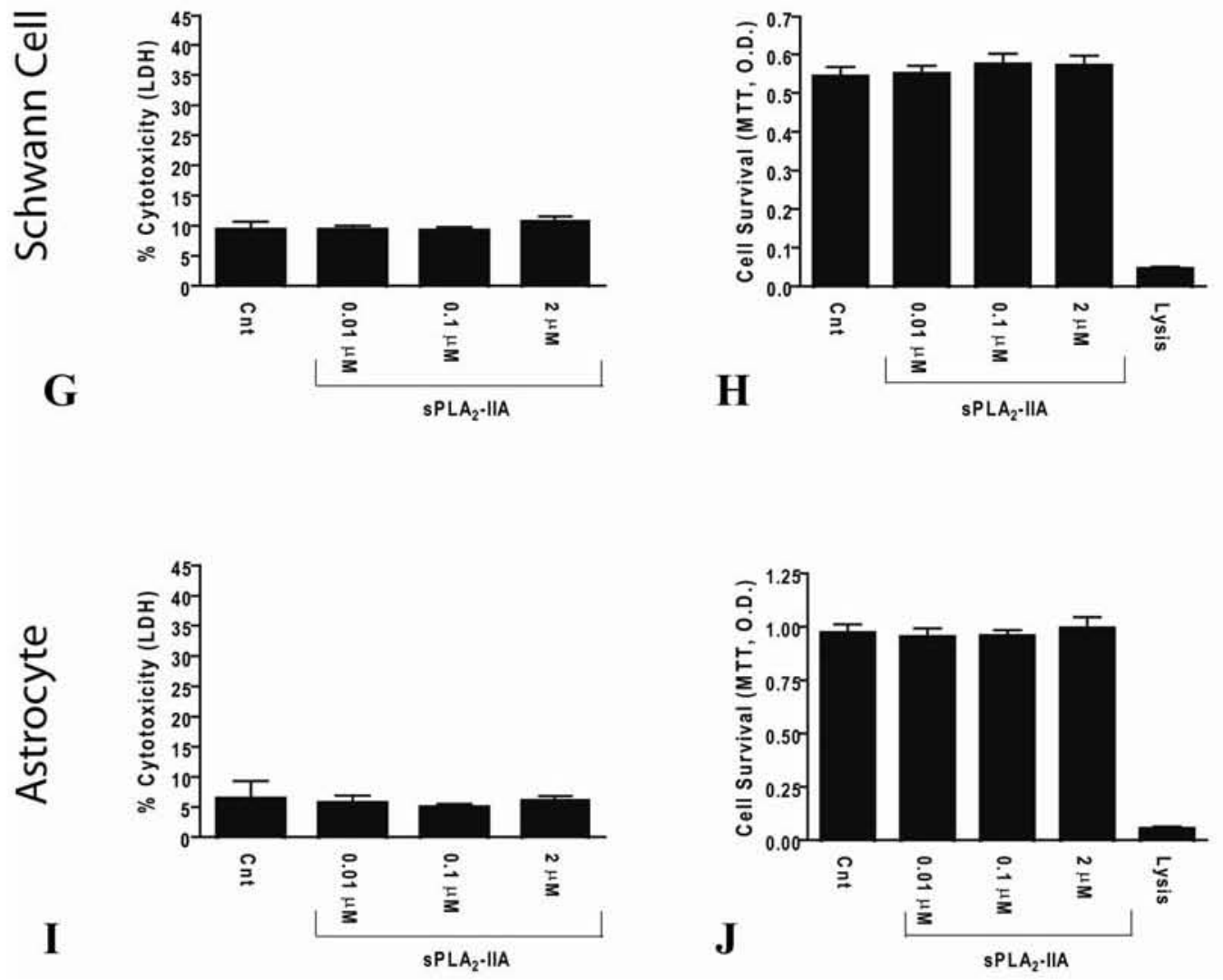
sPLA 2 attenuates $\mathrm{H}_{2} \mathrm{O}_{2}$ induced oligodendrocyte death in vitro.

To address what effects injury induced SPLA 2 -IIA expression might have on oligodendrocytes, an in vitro Hydrogen peroxide $\left(\mathrm{H}_{2} \mathrm{O}_{2}\right)$ injury model was developed. When adult oligodendrocytes were challenged with a 30 min pulse of $\mathrm{H}_{2} \mathrm{O}_{2}$, there was a dose dependent increase in $\mathrm{sPLA}_{2}$-IIA expression over the vehicle control $48 \mathrm{hr}$ after $\mathrm{H}_{2} \mathrm{O}_{2}$ insult (Fig. 11A). Immunoflorescence staining confirmed the presence of sPLA ${ }_{2}-$ IIA-IR in oligodendrocytes in naïve (Fig. 11B-D) and following $\mathrm{H}_{2} \mathrm{O}_{2}$ treatment (Fig. $11 \mathrm{E}-\mathrm{J})$. Interestingly, sPLA $\mathrm{S}_{2}$-IIA staining in naïve oligodendrocytes were homogenously distributed (Fig. 11D insert) whereas in $\mathrm{H}_{2} \mathrm{O}_{2}$ treated cells showed sPLA $\mathrm{A}_{2}$ aggregates in perinuclear puncta (Fig. $11 \mathrm{G} \mathrm{\&} \mathrm{J} \mathrm{inserts).} \mathrm{It} \mathrm{should} \mathrm{be} \mathrm{noted} \mathrm{that} \mathrm{the} \mathrm{cells} \mathrm{showing}$ sPLA 2 -IIA aggregation also show nuclear fragmentation stained by Hoechst 33342, a nuclear dye suggesting apoptosis (Fig. 11G\&J inserts, blue). 
Figure 11. $\mathrm{H}_{2} \mathrm{O}_{2}$ Injury Induces sPLA Oligodendrocytes.

A) Western blots showing a dose dependent increase in sPLA 2 -IIA $48 \mathrm{hr}$ following a 30

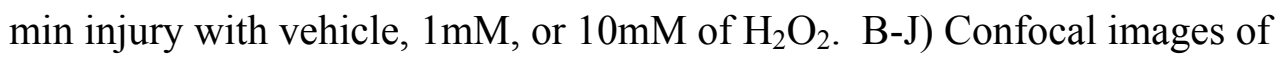
oligodendrocytes $48 \mathrm{hr}$ following treatment with vehicle (B-D) or $10 \mathrm{mM}$ of $\mathrm{H}_{2} \mathrm{O}_{2}(\mathrm{E}-$ J). Dead or dying cells showed either a fragmentation of the nuclei, loss of arborization, and micro-puncta $(\mathrm{E}-\mathrm{G})$ or a round nuclei, swelling of primary processes, and larger puncta $(\mathrm{H}-\mathrm{J})$. Bar: $50 \mu \mathrm{m}$. 

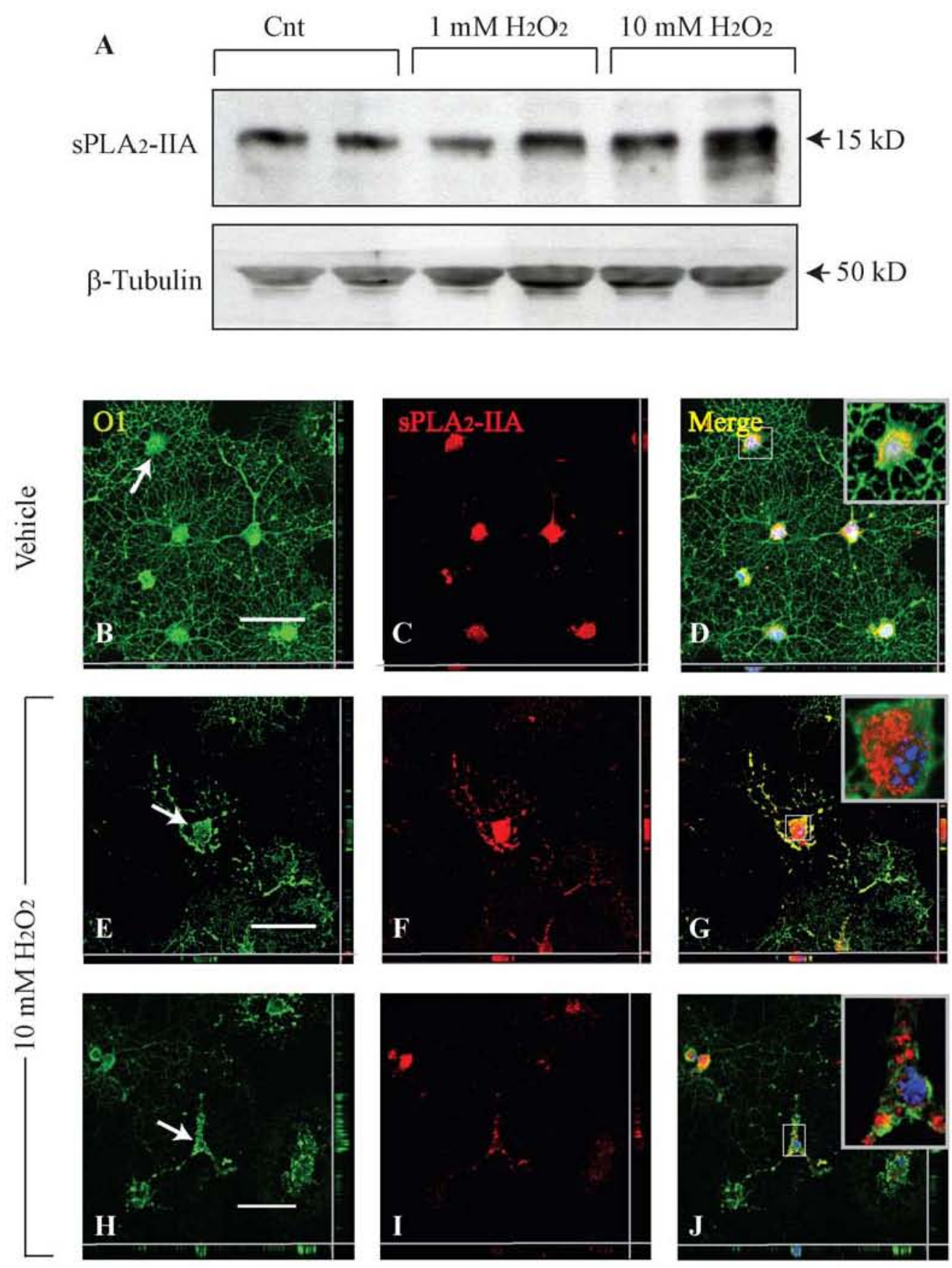
Next, differentiated oligodendrocyte cultures were challenged with either $1 \mathrm{mM}$ or $10 \mathrm{mM} \mathrm{H}_{2} \mathrm{O}_{2}$ for 30 min prior to the administration of various doses of $\mathrm{S} 3319$, a small

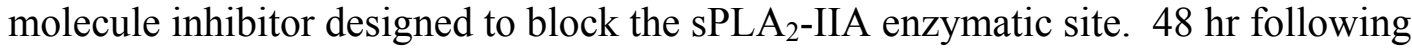
$\mathrm{H}_{2} \mathrm{O}_{2}$ injury, wells containing the sPLA 2 -IIA inhibitor S3319 showed reduced cytotoxicity in both $\mathrm{H}_{2} \mathrm{O}_{2}$ injury intensities (MANOVA $\mathrm{F}_{3,48}=26.63$, $\mathrm{p}<0.0001$; Fig. 12A). In the $10 \mathrm{mM} \mathrm{H}_{2} \mathrm{O}_{2}$ treated wells; cells treated with vehicle showed LDH levels suggestive of $46.3 \pm 9 \%$ cell death as compared to $28.4 \pm 3 \%$ cell death in the S3319 treated wells $(\mathrm{p}<0.001)$. These results suggest that increased $\mathrm{sPLA}_{2}$-IIA enzymatic activity partially mediates $\mathrm{H}_{2} \mathrm{O}_{2}$ induced oligodendrocyte cell death.

Independent of its enzymatic site, the binding of sPLA $\mathrm{A}_{2}$ to its receptor ( $\left(\mathrm{SLA}_{2}-\mathrm{R}\right)$ has been shown to have limited pro-survival functions. To assess the receptors role in cell survival, oligodendrocytes were pretreated with either vehicle or $50 \mu \mathrm{M}$ APMP, a non-catalytic sPLA 2 receptor ligand 30 min prior to the addition of vehicle or $5 \mathrm{mM} \mathrm{H}_{2} \mathrm{O}_{2}$ for $30 \mathrm{~min}$. In contrast to its enzymatic blockade, inhibition of the $\mathrm{SPLA}_{2}-\mathrm{R}$ resulted in increased cell death (ANOVA $\mathrm{F}_{3,44}=364.5, \mathrm{p}<0.0001$, Fig. 12B), approximately $14 \%$ more cytotoxicity compared to the vehicle controls $(59.9 \pm 7.9 \%$ vs. $46.0 \pm 5.3 \%)$. Similarly, when the same cultures were assessed with MTT reduction assay, treatment with APMP reduced the optical density from $0.189 \pm 0.02$ to $0.154 \pm 0.03$ (ANOVA $F_{3,34}$ $=50.79, \mathrm{p}<0.0001$, Fig. $12 \mathrm{C})$. Thus, in contrast to blockade of the enzymatic site which increases cell survival, blockade of the $\mathrm{SPLA}_{2}$ receptor decreased cell survival. 
Figure 12. sPLA Oligodendrocyte Cell Death.

(A). The highest dose of sPLA 2 -IIA inhibitor S3319 $(1.25 \mu \mathrm{M})$ was able to decrease by $17.9 \%$ the amount of cytotoxicity induced by a 30 min treatment with $10 \mathrm{mM} \mathrm{H}_{2} \mathrm{O}_{2}$. Blockade of the sPLA $\mathrm{PL}_{2}$-IIA receptor in contrast appears in increase cell death in both the vehicle and $\mathrm{H}_{2} \mathrm{O}_{2}$ treated oligodendrocytes (B \& C). Following addition of $5 \mathrm{mM}$ of $\mathrm{H}_{2} \mathrm{O}_{2}$, cell cytotoxicity increases by $13.9 \%$ in the APMP treated condition $(\mathrm{p}<0.001, \mathrm{~B})$. Within the same wells, cell viability as measured by MTT reduction reduces by $18.9 \%$ among uninjured cells treated with APMP $(\mathrm{p}<0.001, \mathrm{C})$. Note doted line in $\mathrm{C}$ denotes O.D. of wells that were lysed prior to addition of MTT. $(n=7$ wells per group in A, $n=$ 6 wells per group in B \& C, all in vitro studies repeated in triplicate, mean $\pm \mathrm{SD} ; * * \mathrm{p}<$ $0.01, * * * \mathrm{p}<0.001)$ 
A

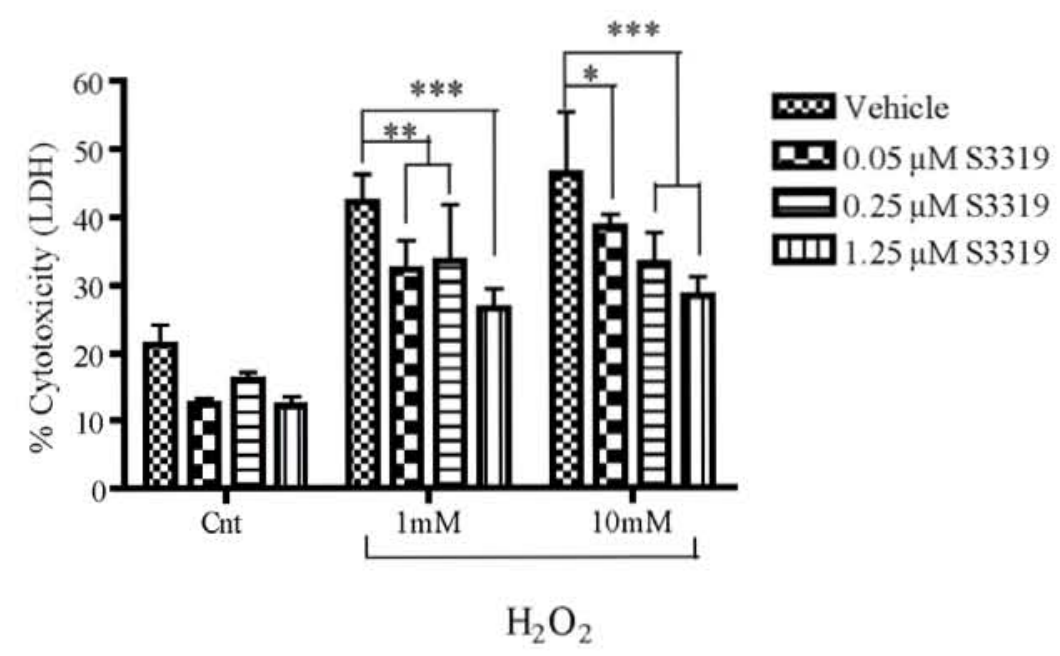

B

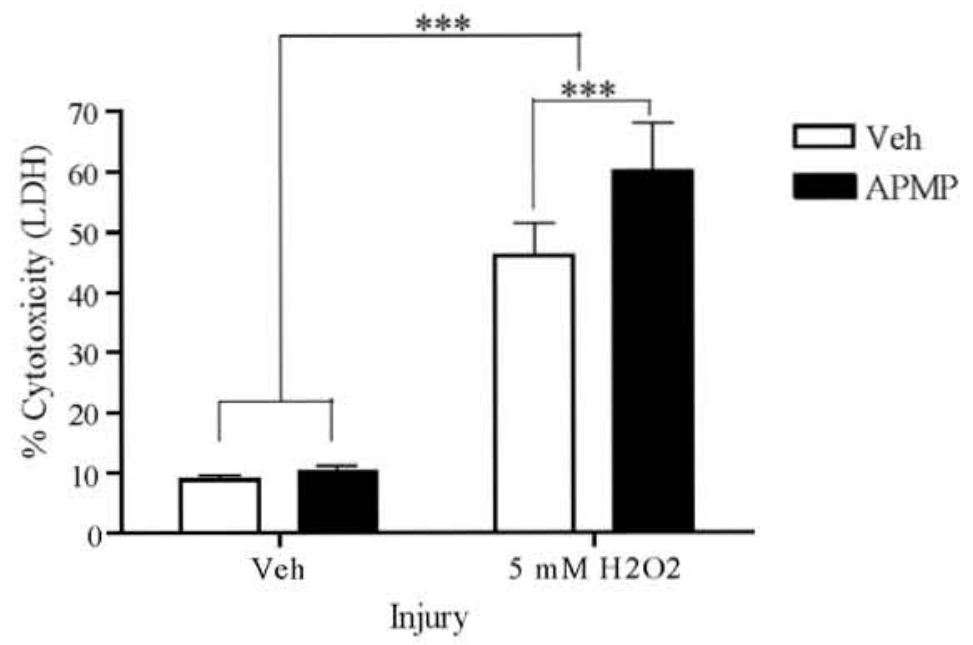

C

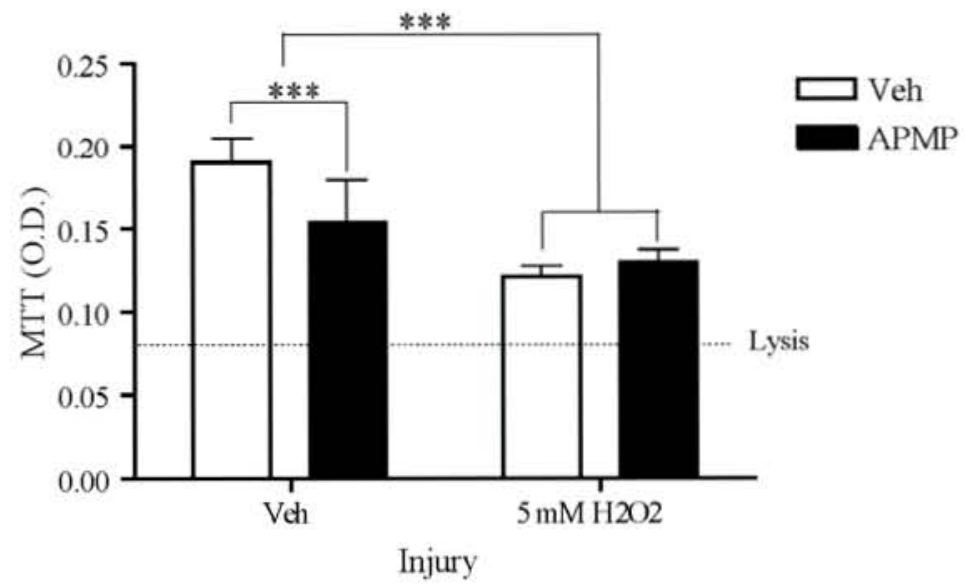


${ }_{\text {SPLA }}$ mediates IL-1 $\beta$ and TNF $\alpha$ induced oligodendrocyte injury in vitro.

To investigate whether the beneficial effects of sPLA $\mathrm{A}_{2}$ blockade mediate more than $\mathrm{H}_{2} \mathrm{O}_{2}$ induced oligodendrocyte injury, we developed a cytokine injury model for oligodendrocyte. IL-1 $\beta$ and TNF $\alpha$ were chosen because both are suggested mediators of secondary SCI (Hostettler and Carlson, 2002, Demjen et al., 2004) and exogenous IL-1 $\beta$ and TNF $\alpha$ induces sPLA 2 -IIA dependent AA release in many cell lines (Kuwata et al., 1998, Kuwata et al., 2000, Kuwata et al., 2005). Similarly, we found that treatment with IL-1 $\beta$ and TNF $\alpha$ created a time dependent increase in PLA $_{2}$-IIA expression in cultured oligodendrocytes (Fig. 13A). When high doses of IL-1 $\beta$ and TNF $\alpha$ were added to oligodendrocyte cultures, pretreatment with S3319 showed a decrease in cytotoxicity, as measured by LDH release after treatment (MANOVA $\mathrm{F}_{1,30}=16.85, \mathrm{p}<0.0001$; Fig. 13H). More dramatic than the observed sparing was the morphological changes associated with this injury model. IL- $1 \beta$ and TNF $\alpha$ treatment results in a dramatic decrease in oligodendrocyte processes extending from the soma at $48 \mathrm{hr}$ (Fig. 13B-D). Interestingly, this process loss was similar to that observed following addition of 0.01 and

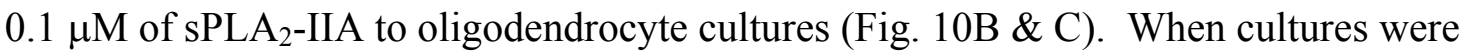
pretreated with S3319, this loss of oligodendrocyte processes was almost fully prevented (Fig. 13E-G). Quantification of area covered by oligodendrocyte processes confirms this observation (MANOVA $\mathrm{F}_{1,30}=9.27, \mathrm{p}<0.01$, Fig. 13I). 
Figure 13. Blockade of sPLA $A_{2}$ in Oligodendrocyte Cultures Prevents Morphological Changes and Death Associated with IL-1 $\beta$ and TNF $\alpha$ Injury.

A) Representative western blot showing time dependent sPLA 2 -IIA expression following treatment with low doses ( 1 and $2 \mathrm{ng} / \mathrm{ml}$ ) or high doses (5 and $10 \mathrm{ng} / \mathrm{ml}$ ) of IL-1 $\beta$ and TNF $\alpha$. B-G) Phase contrast images showing oligodendrocyte cultures $72 \mathrm{hr}$ after injury with either vehicle, low doses ( 1 and $2 \mathrm{ng} / \mathrm{ml})$ or high doses ( 5 and $10 \mathrm{ng} / \mathrm{ml})$ of IL-1 $\beta$ and TNF $\alpha$. Cultures were either pretreated with vehicle (B-D) or $1.25 \mu \mathrm{M} \mathrm{S3319}$ (E-G). Note the decrease in oligodendrocyte process extension. H) IL-1 $\beta$ and TNF $\alpha$ injury resulted in very mild cytotoxicity at $72 \mathrm{hr}$, but there was a significant sparing when pretreated with the sPLA 2 inhibitor S3319. I) Quantification of process area in (B-G) showing that $1.25 \mu \mathrm{M} \mathrm{S} 3319$ significantly prevents the destruction of oligodendrocyte processes after IL-1 $\beta$ and TNF $\alpha$ injury. 
A $\quad$ Veh $\quad 3 \mathrm{~h} \quad 6 \mathrm{~h} \quad 12 \mathrm{~h} \quad 24 \mathrm{~h} \quad 48 \mathrm{~h}$

SPLA2-IIA

$\beta$-tubulin

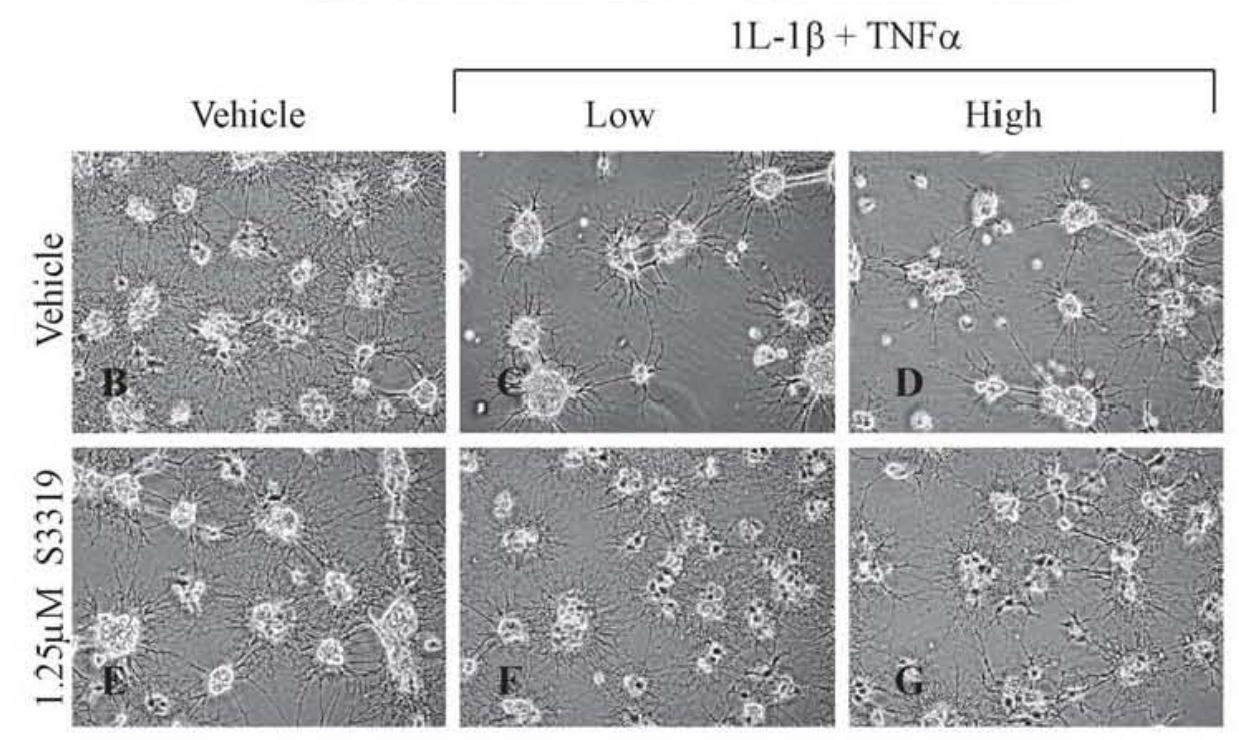

H

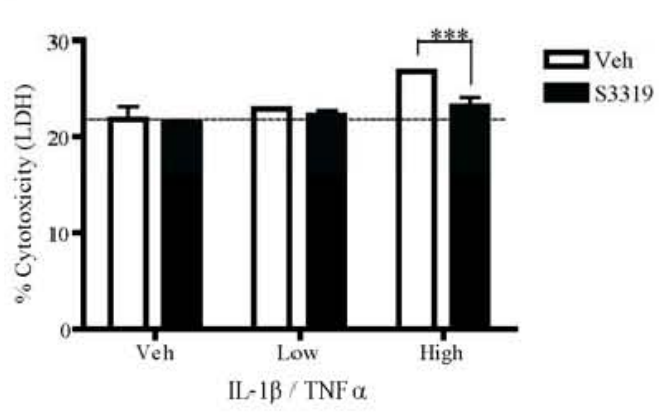

I

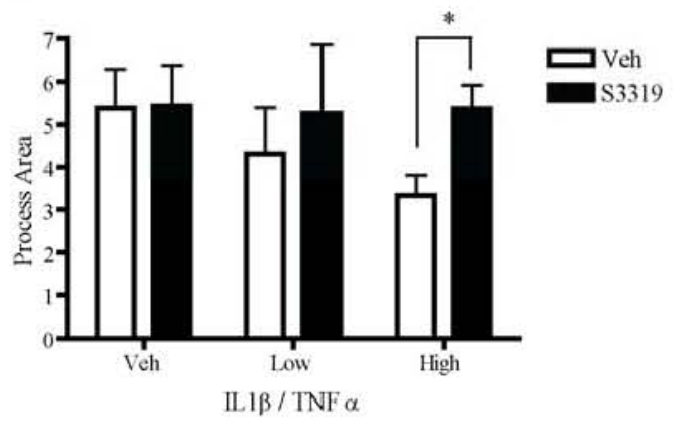




\section{DISCUSSION}

$\mathrm{sPLA}_{2}$ expression following SCI

We previously showed that $\mathrm{cPLA}_{2}-\mathrm{IV} \alpha\left(\mathrm{cPLA}_{2} \alpha\right)$ protein expression increased following SCI and peaked at 7 days post injury. However, measurements of total PLA 2 enzymatic activity in spinal cord homogenates, while increasing after injury, peaked at 4 hr (Liu et al., 2006). This led us to believe that PLA 2 isozymes, other than group IV, most likely contribute to the increase in phospholipases activity observed following SCI. To this end we used RT-PCR to screen for which mammalian isoforms of sPLA $\mathrm{A}_{2}$ were expressed in the naïve and contused spinal cord at $3 \mathrm{hr}$ post injury. We found that of the seven isoforms expressing mRNA, two were up regulated (IIA and IIE), one was down regulated (X), and four were unchanged (IB, IIC, V and XIIA). These results were further confirmed at the protein level with a peak expression at $4 \mathrm{hr}$ which coincides with both apoptosis following SCI (Liu et al., 1997) and the time of peak phospholipase $\mathrm{A}_{2}$ activity following SCI (Liu et al., 2006). Next we showed that IIA and IIE increased within the lesion area and that groups IB, IIA, and IIE were present in oligodendrocytes as well as axons and neurons while group $\mathrm{X}$ was only found extracellularly.

The presence of $\mathrm{SPLA}_{2}$-IB, IIA, IIC, IIE, V, and X is well established within the mammalian brain (Molloy et al., 1998, Suzuki et al., 2000, Kolko et al., 2004) as is upregulation of certain isoforms following CNS injury (Kolko et al., 2004, Kolko et al., 2005, Adibhatla and Hatcher, 2007). However, only one study has demonstrated sPLA $2^{-}$ IIA and V protein in naïve spinal cords (Svensson et al., 2005). Therefore this was the first study to document differential changes of sPLA $_{2}$ isoforms following CNS trauma. 
Interestingly, in separate studies sPLA 2 -IB increased following KA injection and electroconvulsive shock (Kolko et al., 2005), IB, V, and X increased after retinal damage (Kolko et al., 2004), and IIA increased following cerebral ischemia (Yagami et al., 2002b, Lin et al., 2004, Adibhatla and Hatcher, 2007). In comparison, this study found only the group II enzymes increased, while no change was observed in IB, and group X decreased following traumatic SCI. Consistent with this tight regulation, the promoter region of sPLA 2 -IIA gene contains TATA and CAAT boxes as well as several elements homologous with consensus sequences for binding of transcription factors such as AP-1, C/EBPs, CREB, NF-kB, STAT, and PPAR $\gamma$ (Touqui and Alaoui-El-Azher, 2001). The differential regulation of $\mathrm{sPLA}_{2}$ groups suggests a possible injury and isoform specific induction mechanism and varying cellular functions for $\mathrm{SLAA}_{2}$ isoforms in neuropathogenesis.

In addition to demonstrating an induction of $\mathrm{SPLA}_{2}$-IIA following SCI, we also demonstrated an increase of $\mathrm{sPA}_{2}$ within the membrane fraction of cells and a perinuclear compartmentalization of sPLA - IIA following $\mathrm{H}_{2} \mathrm{O}_{2}$ injury. This is significant since sPLA 2 -IIA, while being a secreted molecule, actually displays extremely low enzymatic activity toward the phosphatidylcholine-rich external membrane of cells because this enzyme cannot bind to the zwitterionic interface. As a result sPLA2-IIA shows a marked preference for anionic phospholipids located on the inner leaflet of the bi-lipid membrane. This results in group II enzymes having a decreased ability to act on the external membranes when added exogenously (Bezzine et al., 2000, Murakami and Kudo, 2001). This is supported by the fact that cytotoxicity was initiated by $\mu \mathrm{M}$ levels of exogenously added sPLA 2 -IIA in this study as opposed to nM levels of sPLA $\mathrm{PL}_{2}$-III 
(unpublished observation), which shows little preference for anionic phospholipids. Recent studies have confirmed that AA release by sPLA $_{2}$-IIA transfected cells occurs within the Golgi compartment following synthesis but prior to its initial secretion to the extracellular fluid or binding to anionic heparin sulfate chains on the cell surface (Mounier et al., 2004). Therefore, these authors believe that our observations of increased mRNA levels and increased protein within the cytosolic membrane fraction, in conjunction with a perinuclear punctuate appearance of sPLA 2 -IIA following injury indirectly supports the prevailing theory of SPLA $_{2}$-IIA synthesis and activation. However, further enzymatic studies will be needed for conclusive confirmation.

sPLA $_{2}$-IIA's effect on oligodendrocytes

Since we demonstrated $\mathrm{SLA}_{2}$ induction following SCI, our next question was whether different injury mediators could induce sPLA2-IIA upregulation in oligodendrocytes and whether blockade of SPLA $_{2}$-IIA could reverse injury induced oligodendrocyte cell death. We narrowed our focus to group IIA because of its acute induction following SCI both within the cord and more specifically in oligodendrocytes; as well as its known association with inflammation (Kudo and Murakami, 2002, Pinto et al., 2003, Thwin et al., 2004, Moses et al., 2006).

Most significantly, $\mathrm{H}_{2} \mathrm{O}_{2}$ or IL-1 $\beta$, and TNF $\alpha$ injury induce sPLA 2 -IIA expression in cultured oligodendrocytes and that blockade of $\mathrm{SPLA}_{2}$ attenuates $\mathrm{H}_{2} \mathrm{O}_{2}$ or IL-1 $\beta$, and TNF $\alpha$ induced cell injury. ROS damage is a pervasive injury mechanism involved not only in SCI (Liu et al., 1999a, Liu et al., 2004a, Park et al., 2004) but also Multiple Sclerosis (Lev et al., 2006), Alzheimer Disease (Reddy, 2006), Parkinson Disease 
(Everse and Coates, 2005), and Huntington Disease (Rego and Oliveira, 2003). It was previously shown that $\mathrm{H}_{2} \mathrm{O}_{2}$ (Richter-Landsberg and Vollgraf, 1998) and AA (Wang et al., 2004) can trigger oligodendrocyte death and that $\mathrm{H}_{2} \mathrm{O}_{2}$ injury utilizes cPLA $\mathrm{A}_{2} \alpha$ and sPLA 2 -IIA for AA release in non-CNS cell lines (Han et al., 2003). However, this was the first study to demonstrate that blockade of sPLA 2 -IIA could partially ameliorate the cytotoxic effects of $\mathrm{H}_{2} \mathrm{O}_{2}$ in oligodendrocytes.

Likewise, IL-1 $\beta$ and TNF $\alpha$ have been known to increase sPLA 2 -IIA expression and trigger AA release from cultured cell lines via sPLA 2 -IIA and $\mathrm{cPLA}_{2}$-IV $\underline{\alpha}$ dependent mechanisms (Mounier et al., 2004, Kuwata et al., 2005). Additionally these cytokines have been shown to damage cultured oligodendrocytes (Selmaj and Raine, 1988, Lee et al., 2000, Takahashi et al., 2003, Buntinx et al., 2004). However, this was the first study to demonstrate that blockade of SPLA 2 -IIA could partially ameliorate the cytotoxicity and morphological damage created by IL-1 $\beta$ and TNF $\alpha$ in oligodendrocytes. It must be noted that although S3319 was developed as a specific inhibitor of sPLA 2 -IIA, its activity against other isozymes has not been fully assessed. This being said, $\mathrm{SPLA}_{2}$ induction in oligodendrocytes following ROS and cytokine injury could be a novel target for therapeutic intervention.

While the contradictory results achieved from the blockade of the sPLA $-\mathrm{R}$ are at first troubling, these findings are not surprising. First, the $\mathrm{SLA}_{2}$ isozymes show varying affinities for the $\mathrm{sPLA}_{2}-\mathrm{R}$ in different species. Within rats, the $\mathrm{sPLA}_{2}-\mathrm{R}$ only binds sPLA 2 -IB (Cupillard et al., 1999) which was present, but not increased following SCI. Secondly, the generalized functions of the $\mathrm{sPLA}_{2}-\mathrm{R}$ are far reaching, often grossly beneficial, and include cell growth (Arita et al., 1991), proliferation (Kinoshita et al., 
1997), and migration (Kanemasa et al., 1992). Finally, others have suggested that sPLA $2_{2}^{-}$ $\mathrm{R}$ might function as a clearance mechanism for extracellular $\mathrm{sLA}_{2} \mathrm{~s}$ to protect against enzymatic over activity (Lambeau and Lazdunski, 1999, Yokota et al., 2001).

In this study we chose to focus on the direct cytotoxicity of $\mathrm{SPLA}_{2}$ in isolated oligodendrocytes. A second, equally compelling, yet unexplored hypothesis is that sPLA $_{2}$ could increase secondary SCI not merely by direct cytotoxicity, as demonstrated here, but also by exacerbating the recruitment of neutrophils and macrophages to the injury site which has been previously reported (Popovich et al., 1997). Following sPLA 2 hydrolysis, phospholipids generate a free fatty acid, such as arachidonic acid (AA), and a lysophospholipid such as lysophosphatidyl choline (LPC, a.k.a. lysolechithin). AA can later form epoxides via the cytochrome P450 pathway, leukotrienes via the lipoxygenase pathway, or thromboxanes or prostaglandins via the cyclooxygenase pathway. Many of these products, such as prostaglandin $\mathrm{E}_{2}\left(\mathrm{PGE}_{2}\right)$, can subsequently act as potent chemoattractants that increase the endogenous immune response (Tonai et al., 1999, Resnick et al., 2001). Furthermore, LPC has been shown to transiently demyelinate both the central and peripheral nervous system (Blakemore et al., 1977) and act as a proinflammatory chemoattractant for macrophages (Lauber et al., 2003). Taken together, sPLA $_{2}$ could have detrimental effects on the spinal cord by two means. First, sPLA 2 -IIA could directly induce tissue damage as we have shown here in isolated oligodendrocytes. Secondly, sPLA 2 -IIA could increase inflammation following SCI and exacerbate secondary spinal cord injury, a hypothesis that is under current investigation. The results presented here suggest that the expression of sPLA $\mathrm{A}_{2}$, particularly IIA, which is seen following SCI, might participate in oligodendrocytes death mediated by several 
cytotoxic pathways. More importantly the blockade $\mathrm{SLAA}_{2}$, could provide a crucial therapeutic intervention for not only SCI but other CNS injuries in which $\mathrm{H}_{2} \mathrm{O}_{2}$, IL-1 $\beta$ and TNF $\alpha$ mediate damage. Whether inhibition of $\mathrm{sPAA}_{2}$ following $\mathrm{SCI}$ in vivo creates histological and functional sparing is under active investigation. 
CHAPTER III

\section{SPLA $_{2}$ INJECTIONS INTO THE NAÏVE SPINAL TISSUE RESULT IN A DOSE DEPENDENT LOSS OF FUNCTION AND TISSUE DAMAGE}

\section{INTRODUCTION}

After confirming that $\mathrm{PLA}_{2}$ presence in the naïve spinal cord and increases in oligodendrocytes following SCI, we next determined what effect an increase in $\mathrm{SPLA}_{2}$ could have on the naïve spinal cord. PLA 2 hydrolysis of phospholipids generates a free fatty acid, such as arachidonic acid (AA), and a lysophospholipid such as lysophosphatidyl choline (LPC, a.k.a. lysolechithin). AA can later form epoxides via the cytochrome P450 pathway, leukotrienes via the lipoxygenase pathway, or thromboxanes or prostaglandins via the cyclooxygenase pathway. Many of these products, such as prostaglandin $\mathrm{E}_{2}\left(\mathrm{PGE}_{2}\right)$ can subsequently act as potent chemoattractants increasing the endogenous immune response and subsequent secondary damage. Furthermore, LPC has been shown to transiently demyelinate both the CNS and peripheral nervous system (PNS) (Blakemore et al., 1977) and has been shown to be a chemoattractant as well (Lauber et al., 2003).

It is well established that following spinal cord injury a breakdown of phospholipids and a release of free fatty acids occurs (Demediuk et al., 1985, Demediuk et al., 1989). To determine whether phospholipases are sufficient to create damage in 
normal CNS tissue, Group III secretory $\mathrm{PLA}_{2}\left(\mathrm{sPLA}_{2}-\mathrm{III}\right)$, an extract of bee venom, was injected into the rat thoracic spinal cord (Liu et al., 2006) which created a broad demyelination at $24 \mathrm{hr}$ post-injection. What still remains unclear how sPLA 2 might affect the fate of axons and glial cells within the demyelinated white matter over time.

Additionally it is unknown whether increased $\mathrm{sPLA}_{2}$ within the spinal cord could produce a dose dependent loss of function. Therefore, in this study nano-gram doses of sPLA $_{2}$-III were injected into the rat cervical spinal cord to induce confined lesions in the dorsolateral funiculus (DLF) to better understand the time course of functional and histological damage. It was anticipated that $\mathrm{SPLA}_{2}$-III would create a dose dependent demyelination of the spinal cord, as well as destroy oligodendrocytes, astrocytes, and axons within the lesion area. Accordingly, this dose dependent pathology should produce a graded loss of forelimb function as measured by both pellet retrieval and footprint analysis.

\section{MATERAILS AND METHODS}

A total of 47 female Sprague-Dawley rats (Harlan Sprague Dawley, Inc.), 190$220 \mathrm{~g}$, were used in this study (Table 3). All behavior testing, surgical interventions and postoperative animal care were performed in accordance with the Guide for the Care and Use of Laboratory Animals (1996) and were approved by the University of Louisville Institutional Animal Care and Use Committee. 
Table 3. Experimental groups

\begin{tabular}{ccccc}
\hline Groups & 1 week & 2 weeks & 3 weeks & 4 weeks \\
\hline Sham & 3 & 3 & 3 & 4 \\
Vehicle & 3 & 3 & 3 & 4 \\
$1.5 \mathrm{ng}$ & - & - & - & 4 \\
$3.0 \mathrm{ng}$ & 3 & 3 & 3 & 4 \\
$6.0 \mathrm{ng}$ & - & - & - & 4 \\
\hline Behavior & No & No & No & Yes \\
\hline
\end{tabular}

\section{Pellet Retrieval Acquisition and Assessment}

Twenty female rats were diet restricted to promote acquisition of the pellet retrieval behavior. Animals were intermittently fasted on alternate-nights for $18 \mathrm{hr}$ and allowed ad libitum feeding on weekends. Food was removed from the cages at 5:00 p.m. prior to randomized testing between 8 and 11 am the next morning.

The modified pellet retrieval device consisted of a large Plexiglas holding chamber connected to a smaller feeding chamber (Montoya et al., 1990, Montoya et al., 1991) The rat laid on an elevated $(50 \mathrm{~mm})$ platform, removing the need for the injured rat to weight support and any interference by truncal or hindlimb dysfunction (Onifer et al., 1997). Unlike the original "Staircase Test" (Montoya et al., 1990, Montoya et al., 1991), each rat in the present study randomly used its left or right forelimb to retrieve pellets from the simultaneously loaded left or right feeding tubes instead of from stairs on either side of the elevated platform. Rats reached $1 \mathrm{~cm}$ into shoulder-high feeding tubes to grasp chocolate-flavored dustless precision purified food pellets (45 mg, Bio-Serv, Inc., Frenchtown, NJ). The contralateral limb was prevented from reaching into the opposite tube by extension of their inner wall. Removal of the staircase allowed the rats 
to use the same movement for each pellet retrieved, instead of different movements for retrieving pellets on different stairs. It also allowed rats to eat as many pellets as possible within the testing period (mean $=63.4$ at baseline) rather than just 24 pellets as in the “Staircase Test" used by Onifer and colleagues (Onifer et al., 1997). This increased volume should allow for increased test sensitivity. Care was taken to insure that animals unable to perform the task were given the same level of reward in their home cage following testing.

Once limb dominance was determined, the dominant limb was exclusively used for further acquisition training, injury, and testing. At the end of each acquisition trial, an observer blinded to the treatment groups calculated the percentage of pellets retrieved by dividing the difference between the number of pellets retrieved from the feeding tube and the number remaining on the floor of the feeding chamber by the number of pellets retrieved. Careful attention was given to dismiss any pellet that was eaten from the floor of the chamber. This consisted of $<1 \%$ of all pellets retrieved from the feeding tubes since pellets typically fell out of reach. The objectivity of this score was viewed as a benefit over more commonly used behavior assessments. All rats were given two 5-min training sessions a day for 10 days. Once a rat met training criteria, training was reduced to one session per day. The training criteria were defined as at least 20 pellets retrieved from the feeding tube within 5 minutes with at least $70 \%$ of pellets successfully eaten. One animal that failed to reach training criteria within the 10 day training period was removed from the study. On the last day of the training period, baseline data was collected. After surgery, the rats were tested without diet modification for one session on 
day 3 and weekly for 4 weeks. Sessions were videotaped and images presented were enhanced with Adobe Photoshop for clarity.

\section{Footprint Analysis}

Following pellet retrieval testing, walking track footprint data was collected. Its analysis was modified from the method of de Medinaceli and colleagues (de Medinaceli

et al., 1982). The animal's forepaws were dipped in blue dye (nontoxic). Hindpaws were not evaluated since pilot studies showed only minor behavioral changes and only inking the forepaws increased print clarity. The rats moved from a brightly illuminated starting box to a darkened box containing a food reward by walking through a narrow Plexiglas trough $(8.7 \mathrm{~cm}$ wide by $60 \mathrm{~cm}$ long). Three separate traverses of the track (trials) were recorded per testing session. The trough provided unimpeded movement with ample room on both sides and above the animal. Trials were excluded and repeated if the animal touched the wall of the trough or if the animal turned around at any point. Additionally, two marks were placed $40 \mathrm{~cm}$ apart, centered on the trough, to ensure the rat footprints were not analyzed during the beginning (acceleration) or ending (deceleration) of the task. To insure constant velocity, the rats were timed as they passed from mark 1 to mark 2. If the animal failed to complete the test within 1-2 sec or paused while traversing the trough, the trial was excluded from analysis and repeated. Each animal (observer was blind to treatment conditions) performed one training session without paws being dipped in dye, followed by two separate baseline testing sessions on three consecutive days prior to surgery. After surgery, animals were tested on day 3 and weekly for 4 weeks. The footprints were scanned, and digitized images were measured 
using Photoshop version 6.0 (Adobe Systems, Ottawa, Ontario, Canada). Digit spread was measured as the distance between the second digit and fourth digit of the forepaw. Angle of forepaw rotation was measured as the angle made by the third digit, center of the footpad, and a line parallel to the direction of travel. Forepaw length was the distance from the tip of the third digit to the furthest spot on the back of the forepaw print. Stride length was measured from the center of the footpad to the next footpad from the same forepaw. Stance width was determined by drawing two lines connecting two consecutive right forepaw prints and two consecutive left forepaw prints and then measuring the distance between these two lines at the midpoint. To assess digit spread, the maximal digit spread index (DSI) was calculated according to the Brown Toe Spread Index formula (Brown et al., 1989): DSI = $($ EDS - NDS $) / N D S$, where EDS is experimental digit spread, and NDS is normal digit spread. Similar to digit spread, an index was used to calculate angle of forepaw rotation, forepaw length, stride length, and stance width. Results for each parameter are reported as a percentage of baselines. At least three footprints per side were assessed to determine the value for the trial and the 3 trials were averaged to determine the values of each parameter assessed per session.

\section{sPLA - III Injection}

Following behavior acquisition training and baseline behavior assessments, the 19 trained rats were allowed ad libitum food for $48 \mathrm{hr}$ to return to control weight. Trained rats were randomly assigned to the following groups. Animals received either a sham operation ( $\mathrm{n}=3$ ) or injection of $0.2 \mu 1$ of Hank's Balanced Salt Solution (Invitrogen Corp., Carlsbad, CA) containing no sPLA - III (vehicle control), 1.5, 3, or 6 ng of sPLA s- $^{-}$ 
III extracted from bee venom (Cayman Chemical Co., Ann Arbor, MI) unilaterally into the C2/C3 spinal cord ( $n=4$ per injection group, Table 3 " 4 weeks"). Similarly an additional 27 animals that did not undergo behavioral assessments were randomly assigned to sham, vehicle injection, or $3 \mathrm{ng}$ of $\mathrm{sPLA}_{2}$-III injection groups and sacrificed

at either 1,2 , or 3 weeks post surgery $(n=3$ per group per sacrifice time; See Table 1$)$. sPLA $_{2}$-III, a commercially available sPLA 2 , was used because its enzymatic activity has been we characterized. Injection doses were determined by estimating the mass of $\mathrm{SPLA}_{2}$-III that produced enzymatic activity equal to the increase in phospholipases activity seen $4 \mathrm{hr}$ following contusive spinal cord injury (Liu et al., 2006).

Surgical anesthesia was induced with sodium pentobarbital $50 \mathrm{mg} / \mathrm{kg}$, i.p. (Henry Schein, Indianapolis IN). After exposure and stabilization of the spinal column using a device developed at the University of Louisville, the ligament of flavum and intervertebral ligaments were removed between the $\mathrm{C} 2$ and $\mathrm{C} 3$ vertebrae to expose the dorsal spinal cord surface. The dura was carefully opened then, using a stereotaxic apparatus, a beveled glass micropipette (external diameter, 10-20 $\mu \mathrm{m}$ ) was lowered 1.3 $\mathrm{mm}$ lateral to the midline and $0.8 \mathrm{~mm}$ ventral to the pial surface. Next, $0.2 \mu \mathrm{l}$ of vehicle or $\mathrm{SPLA}_{2}$-III was injected into the spinal cord by a PV800 Pneumatic Pico Pump (World Precision Instruments Inc., Sarasota, FA). The needle was left in place for 30 seconds to allow for diffusion into the surrounding tissue.

\section{Tissue Preparation}

After 1, 2, 3, or 4 weeks post-surgery, the rats underwent transcardial perfusion with 4\% paraformaldehyde in PBS. A $2 \mathrm{~cm}$ cervical spinal cord segment containing the 
injection site and a similar one from sham operation rats was cryoprotected in 30\% sucrose, embedded, and cut transversely at $40 \mu \mathrm{m}$. Eight consecutive sets were created with samples every $320 \mu \mathrm{m}$. Additionally, one animal from each group at the 4 week time point was processed for toluidine blue staining.

\section{Luxol Fast Blue Staining}

One complete set of slides were stained with luxol fast blue for myelin as has been previously reported (Liu et al., 2006). The demyelinated area was identified as white in blue stained sections then measured using an Olympus BX60 microscope (Olympus America, Inc., Mellville, NY) equipped with a Neurolucida system (MicroBrightField, Colchester, VT). The maximal cross-sectional area of demyelination (i.e. lesion epicenter) was determined by identifying the section with the largest area of demyelination within the lateral funiculus. Lesion epicenter was additionally confirmed by identifying the needle tract when present. A section at a similar location was identified in each rat that underwent sham operation or vehicle injection and was used for these measurements.

Three dimensional (3-D) reconstruction and unbiased estimation of the white and grey matter was calculated using Neurolucida software utilizing the Cavalieri method (Michel and Cruz-Orive, 1988). The total volume of spared white matter, gray matter, and lesion were calculated by summing their individual subvolumes (Oorschot, 1994). Individual subvolumes of spared tissue were calculated by multiplying the cross-sectional area $\mathrm{A} \mathrm{x} \mathrm{d}$, where $\mathrm{d}$ represents the distance between sections $(320 \mu \mathrm{m})$. 


\section{Immunostaining}

Immunostaining was performed on sections at the lesion epicenter and $320 \mu \mathrm{m}$ rostral, and caudal to the epicenter of each animal. Three sections at similar locations in each rat that underwent sham operation or vehicle injection were used. A mixture of mouse anti-SMI-31 (1:1000; Covance Research Products, Berkeley, CA) and rabbit antiglial fibrillary acidic protein (GFAP; 1:200; Sigma, St. Louis, MO) antibodies or mouse anti-SMI-31 and rabbit anti-P0 (1:300; gift from Dr. J.J. Archelos) antibodies allowed examination of axons, astrocytes, and Schwann cells, respectively. Paired antibody solutions in blocking solution were applied to the sections overnight at $4{ }^{\circ} \mathrm{C}$. On the following day, the sections were incubated with secondary antibodies, goat anti-rabbit FITC and goat anti-mouse Texas Red (1:100; JacksonImmuno) using methodologies previously described (Liu et al., 2006). Hoechst 33342 (10 $\mu \mathrm{g} / \mathrm{ml}$; Sigma) was added to the mounting medium to label nuclei. Primary antibody omission controls were used to evaluate non-specific binding of secondary antibodies. Images were taken using a Nikon Eclipse 90i confocal microscope (Nikon Instruments; Melville, NY).

Axons profiles and Schwann cell myelin rings were quantified within the lesion area of $3 \mathrm{ng}$ animals and the complete DLFs of vehicle and sham operated rats using an Olympus BX60 microscope equipped with Stereo Investigator (MicroBrightField, Colchester, VT). The lesion area, defined by increased cellularity when viewing Hoechst staining and noticeable changes in tissue morphology, was outlined on three separate sections immunostained for SMI-31 and P0, at the epicenter and $320 \mu \mathrm{m}$ rostral and caudal to it. A systematic random sampling grid (vehicle controls $=133 \times 175 \mu \mathrm{m}$, experimental $=127 \times 100 \mu \mathrm{m}$ ) yielded approximately 30 sampling sites per section. All 
profiles were counted under oil with a 100x optical magnification using a $10 \mathrm{x} 10 \mu \mathrm{m}$ unbiased counting square. Axons and myelin rings were counted simultaneously by alternating between Texas red and FITC filters. The number of axon profiles and myelin rings per spinal cord section was estimated using a fractionator probe with a resulting Schmitz-Hof Coefficient of Error $=0.08$ and an average of 212 profiles counted per animal. Results are presented as number of profiles per $\mathrm{mm}^{2}$.

Additionally sections from the lesion epicenter were incubated with mouse monoclonal anti-APC antibody (CC1) (1:1000; Calbiochem, San Diego, CA) overnight at $4^{\circ} \mathrm{C}$. On the second day, the sections were incubated with secondary biotinylated $\operatorname{IgG}$ antibody (1:400; Vector Laboratories, Burlingame, CA) for $1 \mathrm{hr}$ at room temperature. The reaction product was shown by incubation for 5 minutes with $0.02 \%$ diaminobenzidine tetrahydrochloride (DAB) and $0.003 \% \mathrm{H}_{2} \mathrm{O}_{2}$ in $0.05 \mathrm{M}$ Tris- $\mathrm{HCl}(\mathrm{pH}$ 7.6). Non-binding IgG was used in primary antibody omission controls.

\section{Toluidine Blue Staining}

To further examine myelination (Xu et al., 1999a), spinal cord segments from one animal in each of the groups sacrificed at 4 weeks were fixed overnight in a solution containing $2 \%$ glutaraldehyde and $5 \%$ sucrose in $0.1 \mathrm{M}$ sodium cacodylate buffer, $\mathrm{pH} 7.4$, followed by $1 \%$ osmium tetroxide in the same buffer for 1 hour. The tissues were embedded in Spurr's epoxy resin and cured at $70^{\circ} \mathrm{C}$. Transverse semi-thin sections $(1$ $\mu \mathrm{m})$ were stained with a mixture of $1 \%$ toluidine blue and $1 \%$ sodium borate. Images were captured with MicroFire Digital Camera (Optronics, Goleta, California) attached to an Olympus BX60 microscope (Olympus America, Inc., Mellville, NY). 


\section{Statistical Analysis}

One-way analysis of variance (ANOVA) with post hoc Tukey's HSD was used to determine significant differences in histological outcome measures between three or more groups. A repeated measure multiple analysis of variance (MANOVA) was used to analyze behavioral data, followed by Tukey's HSD. Chi square analysis using the Kaplan and Meier method was used to determine significant differences in behavior acquisition curves. Pearson's Product Moment Correlation was used to determine the degree of relatedness between lesion area and behavioral outcome. Data are reported as mean \pm SD except where noted. A $p<0.05$ was considered statistically significant.

\section{RESULTS}

${ }_{\text {SPLA }}$-III Creates a Consistent Demyelination and Remyelination of the Spinal Cord sPLA $_{2}$-III was found to create a consistent and dose dependent lesion when injected into the cervical spinal cord. The maximal cross-sectional area of the lateral white matter demyelination in luxol fast blue-stained sections significantly increased with the dose of sPLA $_{2}$-III injected (ANOVA, $\mathrm{F}_{4,19}=39.785, \mathrm{p}<0.001$, Figs. 14A-C \& Table 4). The lesion area created by $1.5 \mathrm{ng}$ and $3 \mathrm{ng}$ of $\mathrm{sPLA}_{2}$-III was significantly smaller at 4 weeks than the lesions in 6 ng sPLA 2 -III animals upon post hoc analysis (Table 4, p < 0.001). No lesions were found in vehicle or sham-operated groups. After 3-D reconstruction of the lesion with Neurolucida, the lesion volume differed significantly among the three doses of sPLA 2 -III and all injury groups differed from controls (ANOVA, $\mathrm{F}_{4,19}=126.9, \mathrm{p}<0.001$, Figs. 14D-H \& Table 4). Animals injected with $3 \mathrm{ng}$ 
had a significantly larger lesion than animals injected with $1.5 \mathrm{ng}(\mathrm{p}<0.01)$ and a significantly smaller lesion than those injected with $6 \mathrm{ng}(\mathrm{p}<0.001$; Fig $14 \mathrm{H})$.

Similarly, the 1.5 and $3 \mathrm{ng}$ injection groups showed a greater rostral caudal extent of their lesion than the controls and less than the 6 ng group (ANOVA, $\mathrm{F}_{4,19}=76.6, \mathrm{p}<0.001$ ).

Table 4. Demyelination Parameters

\begin{tabular}{|c|c|c|c|c|}
\hline Dose & $\begin{array}{l}\text { Case } \\
\text { (n) }\end{array}$ & $\begin{array}{l}\text { Lesion Volume } \\
\qquad\left(\mathrm{mm}^{3}\right)\end{array}$ & $\begin{array}{c}\text { Max Area } \\
\left(\mu \mathrm{m}^{2}\right)\end{array}$ & $\begin{array}{l}\text { Rostral Caudal } \\
\text { Length (mm) }\end{array}$ \\
\hline Sham & 3 & 0 & 0 & 0 \\
\hline Vehicle & 4 & 0 & 0 & 0 \\
\hline $1.5 \mathrm{ng}$ & 4 & $0.78 \pm 0.23$ & $3.02 \times 10^{5} \pm 0.2 \times 10^{5}$ & $3.04 \pm 1.06$ \\
\hline $3.0 \mathrm{ng}$ & 4 & $1.3 \pm 0.09$ & $5.32 \times 10^{5} \pm 0.7 \times 10^{5}$ & $3.44 \pm 0.31$ \\
\hline $6.0 \mathrm{ng}$ & 4 & $2.4 \pm 0.31$ & $7.82 \times 10^{5} \pm 0.9 \times 10^{5}$ & $5.28 \pm 0.41$ \\
\hline Sig. & & $\mathrm{p}<0.001$ & $\mathrm{p}<0.001$ & $\mathrm{p}<0.001$ \\
\hline
\end{tabular}



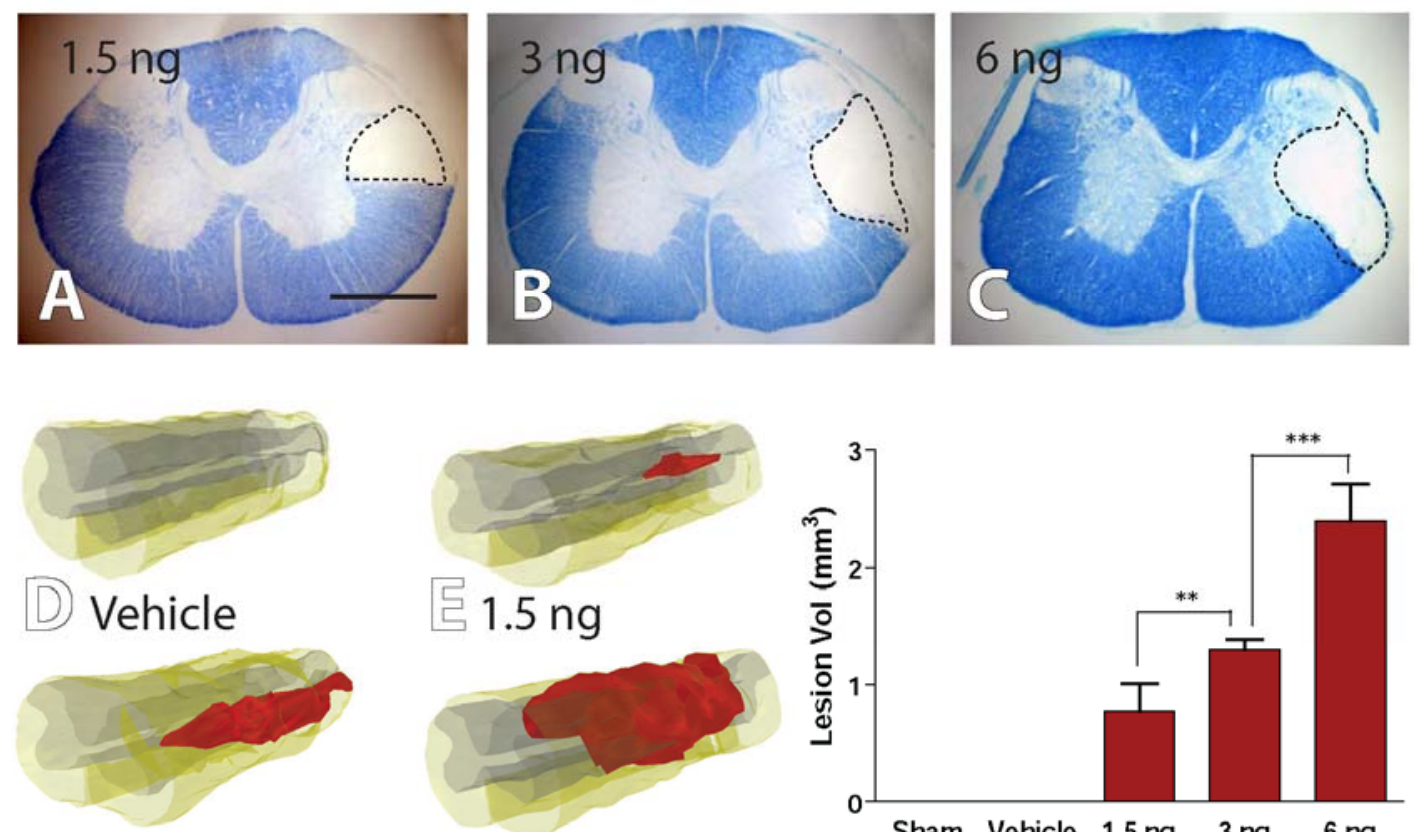

$3 \mathrm{ng}$
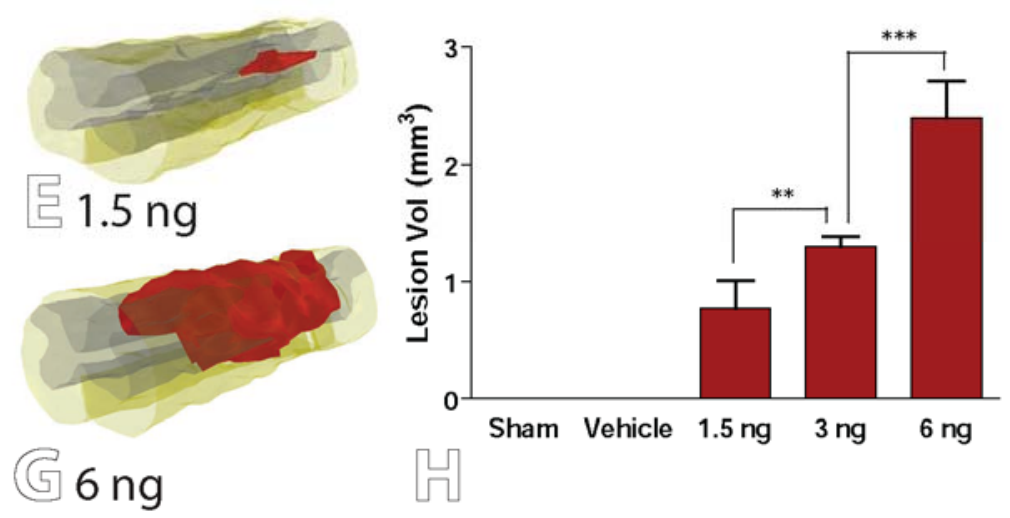

Figure 14. Demyelination Occurring After Unilateral Cervical (C2/3) Spinal Cord

\section{Injections of SPLA 2 -III.}

A-C) Representative sections stained with luxol fast blue for myelin showing graded demyelination at the lesion epicenters 4 weeks post-sPLA 2 -III injections at doses of 1.5 ng (A), $3 \mathrm{ng}(\mathrm{B})$, or $6 \mathrm{ng}(\mathrm{C})$. Dotted line in A-C demarks the lesion area. Note that the lesion is complete within the DLF. D-G) 3-D reconstruction of a $2 \mathrm{~cm}$ segment of Luxol Fast Blue stained spinal cords taken from vehicle (D), $1.5 \mathrm{ng}(\mathrm{E}), 3 \mathrm{ng}(\mathrm{F})$, or $6 \mathrm{ng}$ injected animals (G). H) Graphical representation of 3-D volume reconstructions (D-G) showing significant differences between 1.5 and $3 \mathrm{ng}(* * \mathrm{p}<0.01)$ and $3 \mathrm{ng}$ and $6 \mathrm{ng}$ groups $(* * * \mathrm{p}<0.001)$. Scale bar: A-C $=500 \mu \mathrm{m}$ 
The cell populations within the lesion decreased following spinal cord injury. CC1 staining indicated that there was a marked decrease in mature oligodendrocytes within the lesion (Figs. 15A \& B). Similar to other types of spinal cord injury, an astrocytic border formed at the site of sPLA $_{2}$-III-induced damage. Immunostaining with anti-GFAP antibody revealed a well defined border of reactive astrocytes surrounding the lesion area by 4 weeks with very few astrocytic somas within the lesion (Fig. 15C).

In toluidine blue-stained semi-thin sections, massive demyelination and axonopathy could be seen in the lesion area (Figs. 15D-G). Considerably fewer myelinated axons existed in the lesion at 4 weeks ( 6 ng injection; Figs. 15F \& G) than in the uninjured contralateral white matter (Fig. 15E). While there was less myelin both in the lesion epicenter (Fig. 15F) and in the lesion periphery (Fig. 15G), compared to control regions, myelin was present in all parts of the lesion at 4 weeks post injection with increasing density further from the epicenter. Nuclei were usually associated with myelin rings (arrows in Figs. 15F \& G), suggesting that myelin within the lesion was most likely from Schwann cells and not regenerating oligodendrocytes. Within the lesion, large nucleated granular cells, most likely macrophages, were prevalent (double arrow in Fig. 15F) and blood vessels with their endothelial lining could also be seen (asterisk in Fig. 15G). 
Figure 15. Histological Results Occurring 4 Weeks After Unilateral Cervical (C2/3) Spinal Cord Injections of sPLA 2 -III.

A) Low power image of $\mathrm{CC} 1$ stained section shows the lesion area within the DLF between the ventral horn ( $\mathrm{VH})$ and dorsal horn $(\mathrm{DH})$ B) The high power image shows the clear demarcation of the lesion boarder with a decrease of CC1-positive oligodendrocytes (arrows) within the lesion and a sparing of labeled mature oligodendrocytes outside the lesion. C) Similar to other types of spinal cord injury, there is an astrocytic scar around the lesion confirmed by GFAP labeling (3 ng injury). D-G) Representative toluidine blue-stained semi-thin section at the lesion epicenter of a $6 \mathrm{ng}$ ${ }_{\text {SPLA }}$-III injected animal. Note that at the higher dose (D) there is damage within the ipsilateral dorsal horn and dorsal funiculus (arrow). Letters on the section represent the approximate location of images E-G. E) The intact contralateral lateral funiculus has oligodendrocyte myelinated axons. F) At the lesion epicenter there is a prodigious loss of axons and remyelination of spared axons by Schwann cells (arrows). Many large granular cells (double arrow), most likely macrophages, were also seen. G) At the lesion periphery there are many spared axons that have been almost exclusively remyelinated by Schwann cells (arrows). There were also a high number of blood vessels in the lesion periphery (asterisks). Scale bars: A \& C-D $=500 \mu \mathrm{m}, \mathrm{E}-\mathrm{G}=10 \mu \mathrm{m}$. 

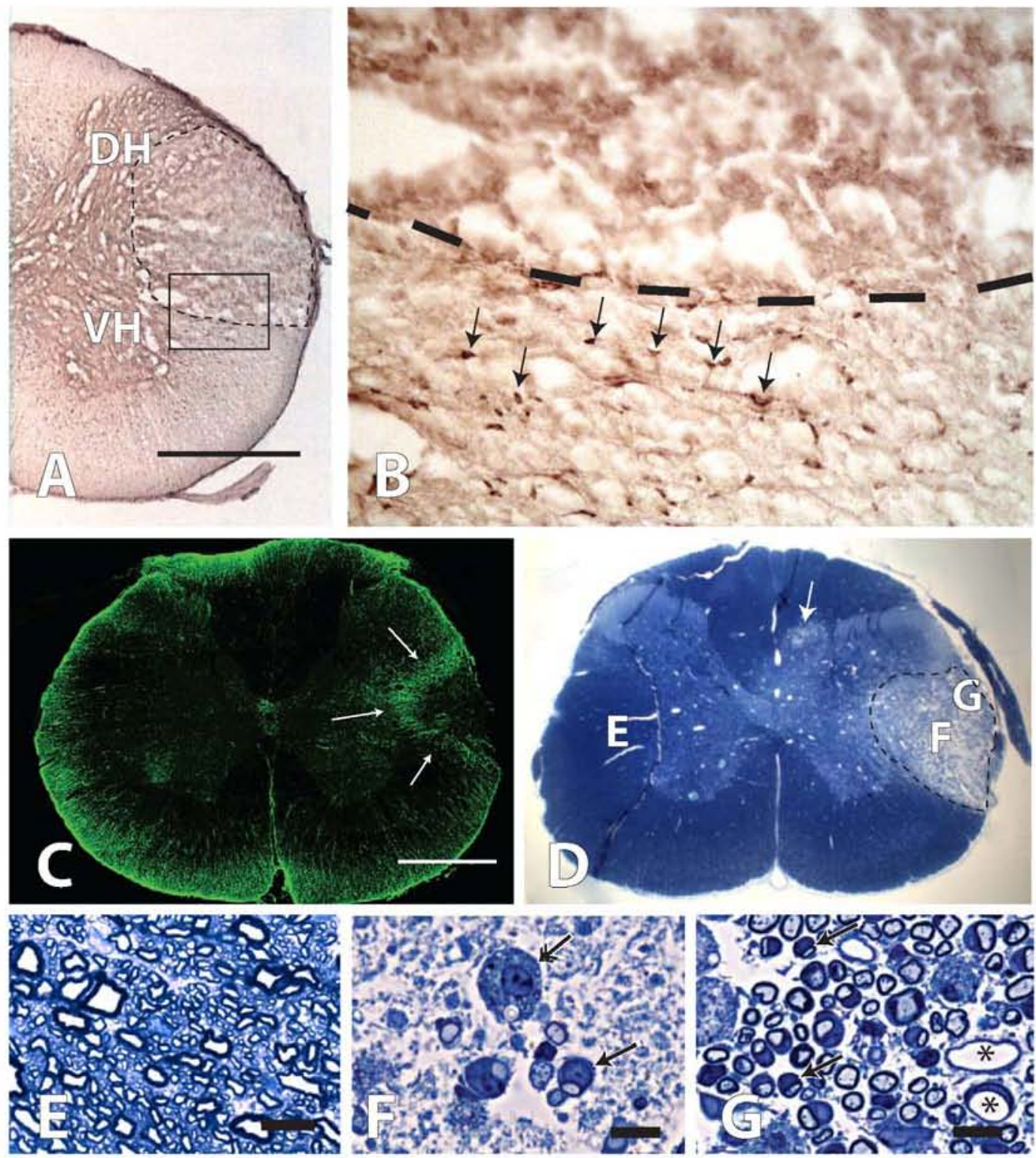
To confirm axonal loss, sections were labeled with anti-SMI-31 (Figs. 16A \& B). The density of axons (number per $\mathrm{mm}^{2}$ ) within the lesion area was then compared to that within the lateral funiculi of vehicle-injected rats. Four weeks after $3 \mathrm{ng}$ injections of sPLA 2 -III, there were $70-77 \%$ fewer axons per $\mathrm{mm}^{2}$ within $\mathrm{sPLA}_{2}$-III lesions ( $3 \mathrm{ng}$ $\mathrm{sPLA}_{2}-\mathrm{III}=30,586 \pm 5,100$; Fig. $16 \mathrm{C}$ ) than in matched areas of vehicle injected controls $\left(120,099 \pm 22,483 ;\right.$ ANOVA, $F_{1,22}=325.1, p<0.001 ;$ Fig. $\left.16 C\right)$. Of note, among the animals that were injected with sPLA 2 -III, the number of axons within the lesion area did not significantly change during the four weeks tested $\left(\mathrm{F}_{3,14}=0.88, \mathrm{p}=0.48\right.$, Fig. 16C) suggesting that axons observed in the fourth week were predominantly original structures and not regenerated. While there was a near significant difference in the density of axons within the vehicle injected animals this is most likely due to increasing total area of the spinal cord as the animals increased in size $\left(\mathrm{F}_{3,14}=3.276, \mathrm{p}=0.063\right.$, Fig. $\left.16 \mathrm{C}\right)$. 

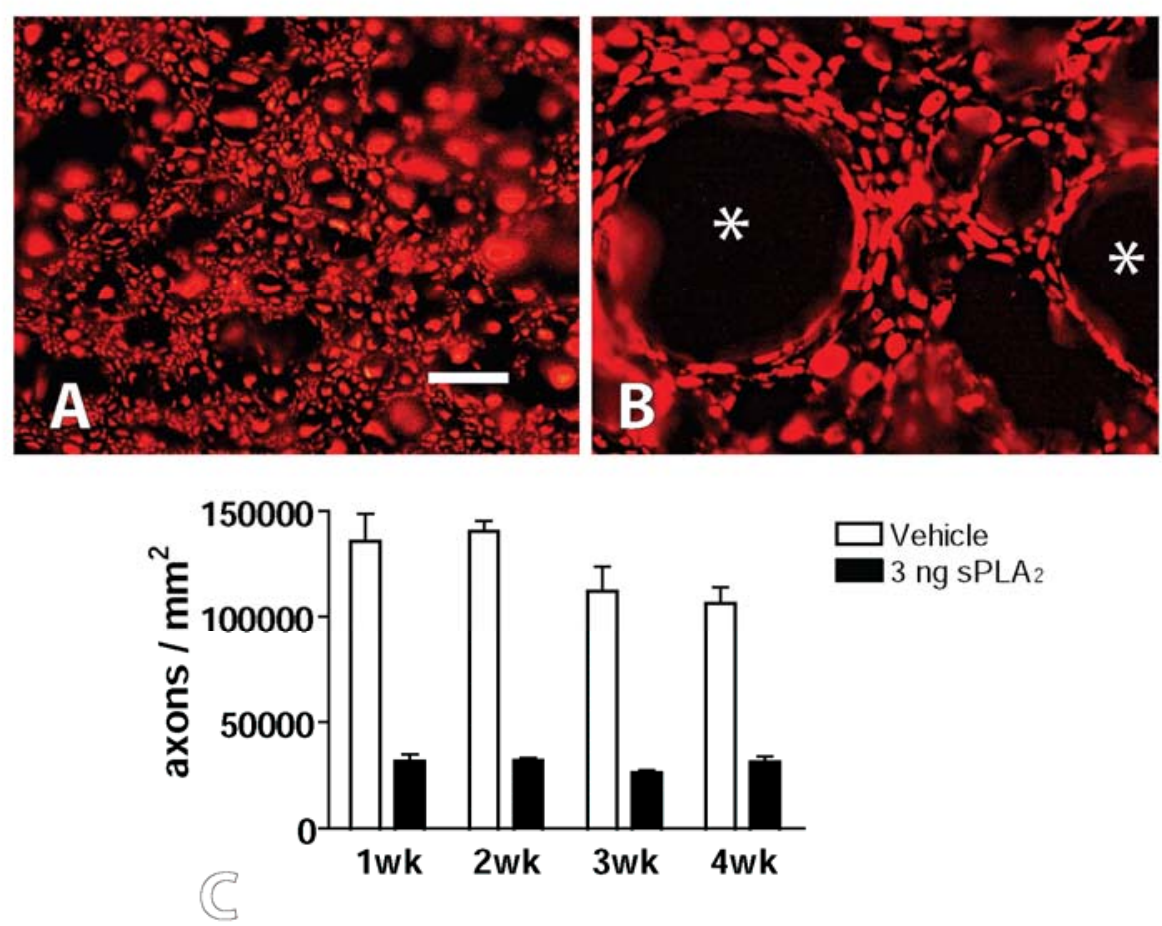

Figure 16. Immunofluorescent labeling and quantification of axons during 4 weeks after unilateral cervical (C2/3) spinal cord injections of sPLA

A, B) Representative SMI-31-immunostained sections in the lateral funiculus of a vehicle control rat (A) and in the lesion area of a rat that received an injection of $3 \mathrm{ng} \mathrm{sPLA} \mathrm{A}_{2}$-III (B) 4 weeks earlier. The circular spaces are most likely micro cyst-like cavities (asterisk in B). C) Quantification of axon loss. Rats that received an injection of $3 \mathrm{ng} \mathrm{sPLA}_{2}$-III had an approximately $70 \%$ decrease in the number of axons per $\mathrm{mm}^{2}$ within the lesion area at 1 week after injury compared to the number of axons in the lateral funiculi of vehicle injected rats. Note that the number of axons within the lesion area remained constant over the period of the study, suggesting that these are spared axons and not regenerated ones. Scale bars: $A \& B=10 \mu \mathrm{m} . \mathrm{C}$, mean $\pm \mathrm{SD} ; \mathrm{n}=3$ animals per group at each time-point. 
To confirm the source of myelin within the lesion, sections were double-labeled with anti-SMI-31 and anti-P0 antibodies (Figs. 17A-F). Positive P0 staining confirmed that myelin within the lesion was from Schwann cells. Confocal images from the lesion epicenter show P0 myelin rings wrapping around SMI-31 axon profiles (Figs. 17D-F). Interestingly, this remyelination was time-dependent. No P0 staining was observed within the lesion area of any animal sacrificed at week 1 . P0 staining was seen in only one of the three animals sacrificed at week 2; however, labeling was extremely weak. There was a dramatic increase in P0 staining within the lesion area by week 3 that persisted at week 4 (ANOVA, $F_{2,11}=17.37, p<0.001$; Fig 17G). Since P0 was only observed around SMI-31 labeled axons, it was estimated that by weeks 3 and 4, 72\% $56 \%$ of axons were myelinated by Schwann cells, respectively (Fig. 17H). 


\section{Figure 17. Immunofluorescent double labeling shows a progressive remyelination of spared axons by Schwann cells over a 4 week period.}

A-C) Representative P0 and SMI-31-immunostained section at the lesion epicenter of a 3 ng sPLA 2 -III injected animal at 4 weeks post injury. A) P0 signaling is strong within the lesion area. Note that P0's specificity for Schwann cell myelin is confirmed by staining of Schwann cells in both the dorsal and ventral roots (white arrows). B) Axons are also present within the lesion area. C) The merged image of A \& B shows that some P0immunoreactive myelin in the lesion area is associated with SMI-31-immunoreactive axons. (D-F) Orthogonal views of confocal images from the 4 week old lesion area of a 3 ng injected animal. Note, the $\mathrm{X} / \mathrm{Y}$ plane is presented in the center panel, $\mathrm{X} / \mathrm{Z}$ plane is presented in the bottom panel, and $\mathrm{Y} / \mathrm{Z}$ plane is presented in the left panel. The area of interest is indicated by green crosshairs in (F). Schwann cell myelin rings, positive for P0 (D), can be clearly seen encircling axons (F), identified by SMI-31 immunostaining (E), within the lesion area. G, H) Quantification of Schwann cell remyelination. G) While no P0 myelin is seen within the lesion area at weeks 1 and 2 after injection of $3 \mathrm{ng}$ SPLA$_{2}$-III, there is a dramatic increase between weeks 2 and 3 that remains constant during week 4. H) The majority of spared axons are remyelinated by Schwann cells by weeks 3 and 4. Scale bars: Scale bars: A-C $=500 \mu \mathrm{m}$; D-F $=5 \mu \mathrm{m}$. G \& H, mean \pm SD; $\mathrm{n}=3$ animals per group at each time-point. 

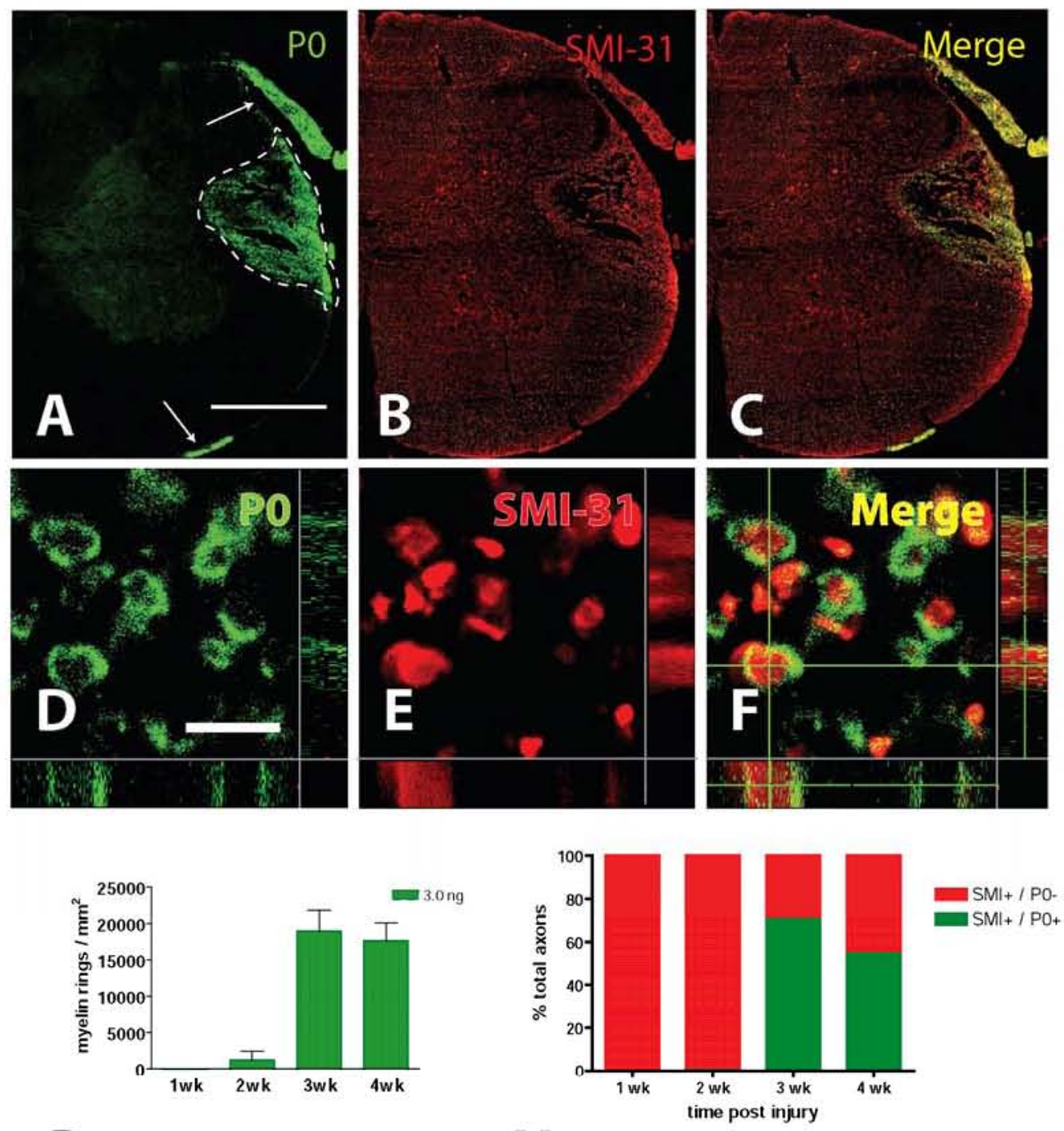

(5)

늠 


\section{sPLA $_{2}$-III Injury Affects Behavior}

Qualitatively, differences existed between the rodents' performance before and after sPLA 2 -III injections. The naïve (Figs. 18A-D), sham operated, and vehicle control rodents retrieved pellets using previously described movements (Mckenna \& Whishaw, 1999) in four stages. First, the animals approached (Fig. 18A), then reached into the feeding tube grasping the pellet with extended digits (white arrow indicates pellet in Fig. 18B). Because of the positioning of the tube and an extension along the inner wall, a rodent could not use its tongue or contralateral limb or scrape the pellet into its mouth. Therefore, the naive rodent grasped the pellet and supinated the closed paw (Fig. 18C). Finally, the rodent completed supination and ate the pellet with the palm facing vertically (Fig. 18D). By 1 week following injections of 1.5 and $3 \mathrm{ng}$ sPLA 2 -III (3 ng rat shown in Figs. 18E-H), these rodents approached the tube (Fig. 18E) and reached (Fig. 18F) in a similar manner to naïve animals. However, they were unable to open the digits, pronate the paw, and close their forepaw around the pellet (Fig. 18G). Upon retraction of the arm, the pellet was dragged from the tube and fell to the chamber floor (Fig. 18H). Two out of four rodents injected with $6 \mathrm{ng}$ were unable to elevate their shoulder to the level of the feeding tube at any time post-injection and so were given a $100 \%$ pellet drop score on each of the testing days. It was found that these $6 \mathrm{ng}$ animals had damage not only of the DLF but also extending into the ipsilateral gray matter and corticospinal tract in the dorsal funiculus (see arrow in Fig. 15D). This loss of function is not surprising since large dose injections into the $\mathrm{C} 2 / 3$ white matter could cause death of interneurons and motorneurons which would correspond with control of the acromiotrapezius and levitor claviculae (McKenna et al., 2000). 
Figure 18. Video captured images of pellet retrieval test performance prior to injury (A-D) and 1 week following 3 ng injection of sPLA - III (E-H).

Forelimb lift (A), reach, forepaw digits open (B), pronation, grasp, and supination (C) movements were required for successful pellet (white arrow in B) retrieval (D) by naïve rats. The 3 ng sPLA 2 -III injected rats could reach into the feeding tubes (E) and open their digits (F) for the pellets (white arrows in F-H). However, they could not grasp them (G). As the pellets were dragged out of the feeding tube, they fell to the floor out of reach (white arrow in $\mathrm{H}$ ). Counts of dropped pellets were used to determine the percentage of pellets retrieved resulting in a quantitative and objective measure of forelimb function. I) Schematic showing the plunger used to deliver a pellet for a rat that is lying on the platform in the feeding chamber and reaching into a feeding tube. 

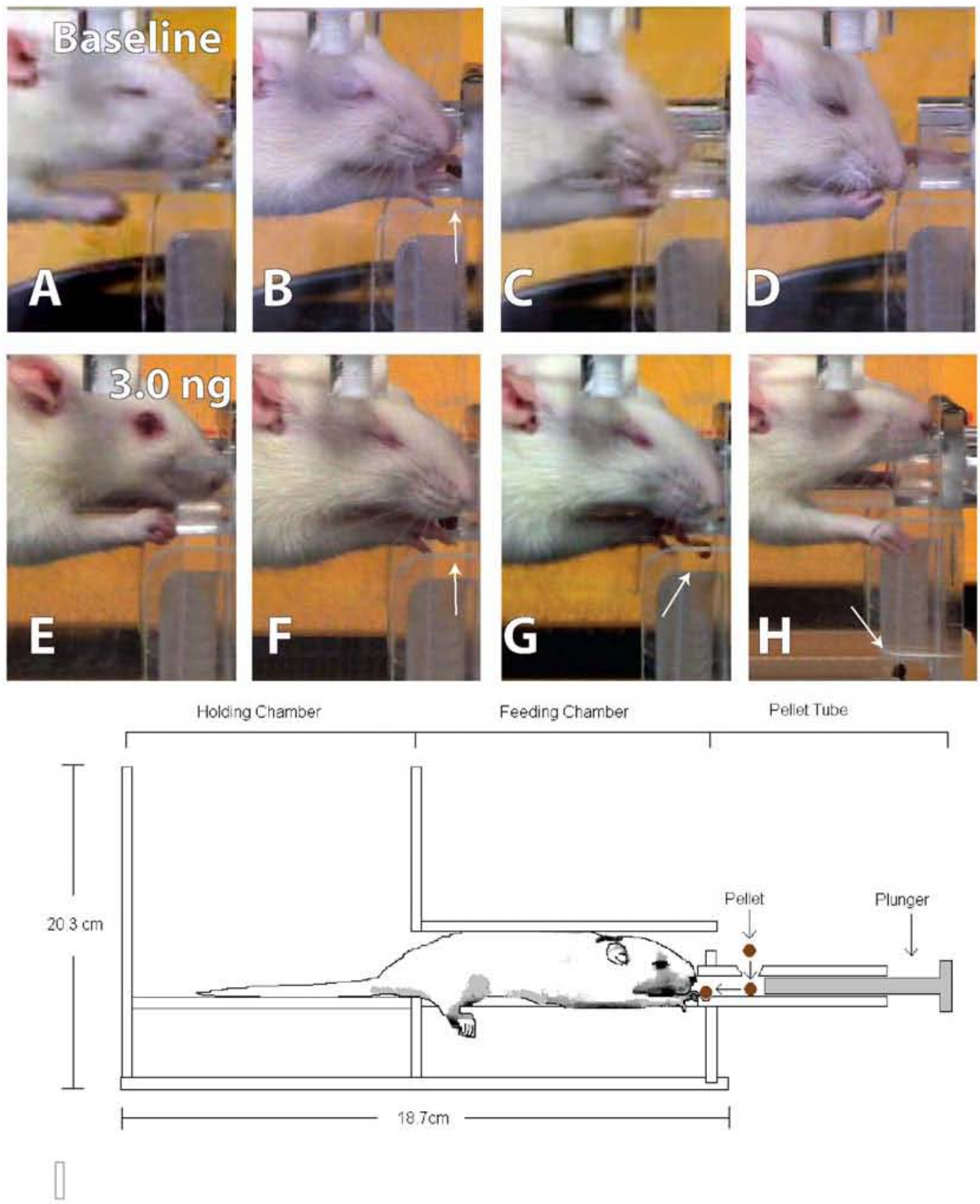
Injection of sPLA $\mathrm{A}_{2}$-III into the cervical spinal cord DLF had a significant quantitative effect on pellet retrieval performance as well (Fig. 19A). A significant main effect was observed between time post-op and the percentage of pellets retrieved (MANOVA, $\mathrm{F}_{2.6,70.3}=22.427, \mathrm{p}<0.001$ ). Tukey's HSD post hoc analysis revealed a significant difference from baseline pellet retrieval in all sPLA $\mathrm{SLII}_{2}$ injected animals. The $6 \mathrm{ng}$ group decreased precipitously on day $3(\mathrm{p}<0.001)$ and never returned to baseline. The 3 ng group showed decreased pellet retrieval on day $3(p<0.001)$ but was statistically indistinguishable from baseline after 3 weeks $(p=0.20)$. Similarly, animals injected with $1.5 \mathrm{ng}$ of $\mathrm{sPLA}_{2}$-III decreased by day $3(\mathrm{p}<0.01)$ but were indistinguishable from baseline levels by 1 week $(\mathrm{p}=0.32)$ or later after injury. No difference was seen in the sham $(p=0.97)$ or vehicle $(p=0.89)$ groups. This general trend of improvement was confirmed by a significant time/group interaction (MANOVA, $\left.\mathrm{F}_{10.4,70.3}=5.104, \mathrm{p}<0.001\right)$ and post hoc analysis revealing that the $3 \mathrm{ng}$ group significantly improved from its day 3 deficit by weeks 3 and $4(\mathrm{p}<0.05)$. Additionally, at day 3 the $6 \mathrm{ng}(\mathrm{p}<0.001), 3 \mathrm{ng}(\mathrm{p}<0.001)$, and $1.5 \mathrm{ng}(\mathrm{p}<0.05)$ rodents performed significantly worse than the vehicle injected animals. Afterwards this pattern continued for the $6 \mathrm{ng}$ and $3 \mathrm{ng}$ groups with the $1.5 \mathrm{ng}$ group returning to baseline by 1 week. Most importantly the pellet retrieval test detected differences among the sPLA $-\mathrm{III}$ injected animals. For example, at day 3 there was a significant difference between the $1.5 \mathrm{ng}$ injury group and the $6 \mathrm{ng}(\mathrm{p}<0.001)$ and $3 \mathrm{ng}(\mathrm{p}<0.05)$ animals a pattern which continue through all time points. A difference in pellet retrieval was also seen between the $3 \mathrm{ng}$ and $6 \mathrm{ng}$ groups at week $3(\mathrm{p}<0.01)$. Most importantly, the lesion area was 
inversely correlated with the averaged pellet retrieval ability for each animal $\left(\mathrm{R}^{2}=\right.$ 0.7285; Fig. 19B). 


\section{Figure 19. Behavioral results occurring after unilateral cervical (C2/3) spinal cord}

injections of sPLA-III.

A) Only groups injected with $\mathrm{SPLA}_{2}$-III showed decreased pellet retrieval ability at 3 days post injury. The $6 \mathrm{ng}$ rats showed a significant difference from baseline at all time points with $3 \mathrm{ng}$ animals recovering by 3 weeks and $1.5 \mathrm{ng}$ by 1 week post injection. A dose-dependent decrease in pellet retrieval was also observed 3 days post-injection with 3 ng and 6 ng animals. Pellet retrieval performance improved in all injury groups, however, at 4 weeks this was still significantly impaired in the $3 \mathrm{ng}$ and $6 \mathrm{ng}$ groups compared to all other groups. B) The pellet retrieval performance decreased as the lesion area increased. Therefore, this test is sensitive to graded demyelinating and axon injuries in the upper cervical spinal cord lateral white matter. C \& D) The 6 ng sPLA 2 -III injected rodents rotated their forepaw less 3 days post-surgery. Additionally the 6 ng rats maintained this lower rotation through weeks 1 and 2 . At day 3 only the 6 ng animal had a significantly lower angle of rotation than all other groups. At 2 weeks the vehicle and sham animals were both significantly higher than the $6 \mathrm{ng}$ and the $3 \mathrm{ng}$. By 3 and 4 weeks post-injection there was no difference among injured groups. A difference in digit spread was only detected on day 3, with $3 \mathrm{ng}$ and $6 \mathrm{ng}$ animals showing significant narrowing of the digits. E \& F) Representative forepaw prints from either a vehicle (E) or $3 \mathrm{ng}(\mathrm{F})$ right side injection, 3 days post injury. Note the difference in both paw rotation and digit spread. $A, C, D$, mean $\pm S E M ; n=4$ animals per group at each time-point. 

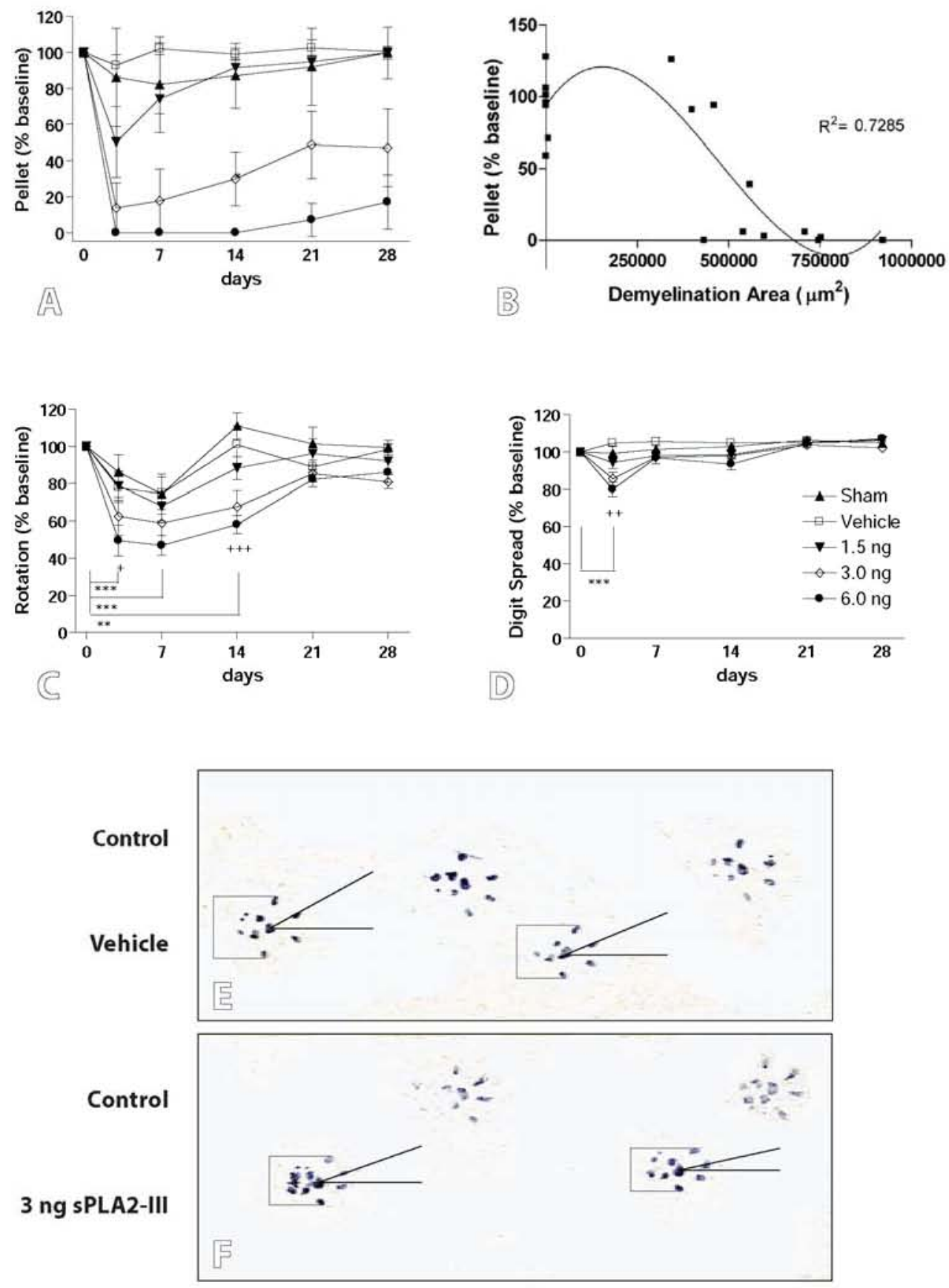
Similarly, footprint analysis could detect behavioral changes following sPLA 2 -III injection but not as well as the pellet retrieval assessment. Of the five footprint analyses, forepaw length (MANOVA, $\mathrm{F}_{4,54}=2.25, \mathrm{p}=0.09$ ), stance width (MANOVA, $\mathrm{F}_{4,54}=$ $0.639, \mathrm{p}=0.637)$, and stride length (MANOVA, $\left.\mathrm{F}_{4,54}=0.460, \mathrm{p}=0.765\right)$ were not significantly different for any group or time point. Forepaw rotation varied significantly over time (MANOVA, $\left.\mathrm{F}_{14,97}=2.04, \mathrm{p}<0.05\right)$ and between groups (MANOVA, $\mathrm{F}_{4,54}=$ $6.11, \mathrm{p}<0.001$; Figs. 19C, E, F). All three injury groups were found to vary significantly from baseline initially but, in contrast to pellet retrieval performance, recovered to baseline by 3 weeks. The 6 ng group differed significantly from baseline at 3 days ( $p<$ $0.001), 1$ week $(\mathrm{p}<0.001)$, and 2 weeks $(\mathrm{p}<0.01)$ post injury. The $3 \mathrm{ng}$ and $1.5 \mathrm{ng}$ group differed from baseline only at 1 week post injury $(\mathrm{p}<0.05)$. Of note, while no significant difference was seen from baseline there was a significant recovery between 1 week and 2 weeks for both sham $(p<0.05)$ and vehicle controls $(p<0.01)$. Significant differences between injury groups were only detected at 3 days $(p<0.05)$ and 2 weeks ( $p$ $<0.001)$ post injury. At 3 days post injury, forepaw rotation in the 6 ng group was significantly worse than sham operated rats $(\mathrm{p}<0.05)$. At week 2 , the $6 \mathrm{ng}$ group was significantly worse than sham $(\mathrm{p}<0.001)$, vehicle $(\mathrm{p}<0.01)$ and $1.5 \mathrm{ng}(\mathrm{p}<0.05)$ animals and the 3 ng group was significantly worse than sham $(p<0.001)$ and vehicle $(p$ $<0.05)$ rats. It is worth noting that the only detectable difference among sPLA 2 -III injected groups was at one time point ( 2 weeks) and between the $6 \mathrm{ng}$ and $1.5 \mathrm{ng}$ groups.

There was a significant difference in the digit spread over time (MANOVA, $F_{13.26 \text {, }}$ $179=4.506, \mathrm{p}<0.001$ ) and between injection groups (ANOVA, $\mathrm{F}_{4,19}=4.168, \mathrm{p}<0.005$; Figs. 19D, E, F). Further analysis of time revealed that all differences were confined to 
day $3(\mathrm{p}<0.001)$. At day 3 , the $3 \mathrm{ng}$ and $6 \mathrm{ng}$ groups were both significantly different from baseline ( $\mathrm{p}<0.001, \mathrm{E}, \mathrm{F})$. A difference among groups could be seen on day 3 ( $\mathrm{p}<$ $0.001)$ and 2 weeks $(\mathrm{p}<0.05)$ following injury. At day 3 , the 6 ng group was significantly narrower than the sham $(\mathrm{p}<0.01)$, vehicle $(\mathrm{p}<0.01)$, or the $1.5 \mathrm{ng}$ groups $(\mathrm{p}<0.05)$. The 3 ng group was significantly narrower than the vehicle group only ( $p<$ 0.05). At two weeks, only a near significant difference existed between the $6 \mathrm{ng}$ and vehicle control groups $(\mathrm{p}=0.06)$. As with forepaw rotation, digit spread detected a significant difference among sPLA 2 -III injected groups at day 3 , but in contrast was unable to detect group differences at 2 weeks.

\section{DISCUSSION}

Initially, this study showed that nanograms of sPLA 2 -III were sufficient to demyelinate the normal rodent spinal cord. Secondly, the previously observed axonopathy was quantified, revealing it to be more pervasive than originally thought, with an approximately $75 \%$ loss of axons. Thirdly, we confirmed that oligodendrocytes and astrocytes die within the lesion area. However, this conclusion is based on one two stains and the fate of precursor cells still remains unclear. Most importantly, for the first time, we observed a prodigious remyelination of $72 \%$ of spared axons by Schwann cells between 2 and 3 weeks after injection of sPLA SIII. $^{2}$

Schwann cells have been shown to remyelinate the spinal cord in small numbers following traumatic SCI in rats (Bresnahan, 1978) and humans (Bunge, 1994). Unfortunately, a large percentage of the spinal cord remains demyelinated after contusion (Cao et al., 2005) suggesting that further remyelination could be beneficial. In previous 
experiments we and others showed that transplanted Schwann cells increased axonal regeneration, were neuroprotective, and improved functional recovery following SCI in rats (Xia et al., 1995, Xu et al., 1995, Xu et al., 1997, Xu et al., 1999b, Takami et al., 2002, Pearse et al., 2004). Given the previous work showing functional recovery following Schwann cell transplantation and the lack of appreciable axon regeneration within the lesion, it is tempting to hypothesize that the observed recovery in this study was due to remyelination by Schwann cells. However, Schwann cells' exact role in functional recovery following $\mathrm{SPLA}_{2}$ injection remains to be investigated.

Schwann cell immigration into the injured human and experimental animal spinal cord is still controversial. Some authors suggest that Schwann cells enter from dorsal and ventral roots and contiguous peripheral nerves (Jasmin et al., 2000). Other groups believe that these cells immigrate either through porous blood vessels (Talbott et al., 2005) or through breaches in the dura (Guest et al., 2005). It has also been suggested that cells possessing a Schwann cell-like phenotype are actually the result of altered maturation of endogenous oligodendrocyte precursors due to the absence of astrocytes (Talbott et al., 2006). The latter hypothesis is particularly intriguing since the present results show a lack of astrocytes within sPLA $\mathrm{PL}_{2}$-III-induced lesions.

Interestingly, sPLA $\mathrm{A}_{2}$-III-induced demyelination bears some striking similarities to other demyelination models. Intraspinal LPC -injection, a common demyelination/remyelination model described by Blakemore and colleagues (Blakemore et al., 1977, Blakemore, 1978, Blakemore, 1982, Jeffery and Blakemore, 1995) also leads to loss of oligodendrocytes, astrocytes, and axons. Moreover, LPC is a product of $\mathrm{sPLA}_{2}$ digestion, providing a logical connection between these two models (Dutta et al., 1979). 
However, this LPC induced remyelination occurs at 2 weeks post injection and is much less robust. Furthermore, when Woodruff and Franklin (1999) compared remyelination following injections of LPC, ethidium bromide, and anti-galactocerebroside antibody into the brainstem, oligodendrocytes dominated the remyelination in all cases with only 10 $40 \%$ attributable to Schwann cells. Further more remyelination occurred at 3.5 weeks, later than in the present study. Finally, these models had greater axonal sparing than was observed after $\mathrm{SPLA}_{2}$-III injections. While these differences could be due to dose variations, they also suggest additional molecular processes might be involved in $\mathrm{SPLA}_{2}$ -induced injury.

There are several advantages to using the current model of sPLA $_{2}$-III induced demyelination over previously mentioned methods. First, the use of phospholipases, which are increased in many CNS disorders, are a closer parallel to natural inflammatory processes than synthetic molecules such as ethidium bromide. Second, we have shown that $\mathrm{SPLA}_{2}$ injections produce a highly focal and controllable lesion that can be used to create a graded lesion of the white matter with little grey matter damage at lower doses. When $\mathrm{SPLA}_{2}$ injections are used in conjunction with the pellet retrieval task one can produce very controlled cervical spinal cord injury model, with low mortality, and a functional outcome measure. Third, previous models have shown only slight P0 remyelination, making this a superior model for investigation of Schwann cell remyelination. However, the presence of $\mathrm{P} 0$ remyelination could be viewed as an unwanted experimental artifact detracting from this models appeal. Another disadvantage is that while sPLA $\mathrm{PL}_{2}$-III does have a potent phospholipase activity; the presence of this particular group has not been shown in the mammalian CNS. 
In this study, the modified pellet retrieval task was a sensitive and quantifiable behavioral measure able to detect both differences in functional loss and recovery following graded sPLA 2 -III-induced cervical DLF lesions. Previously, the "Staircase Test" was used to show forelimb dysfunction without significant recovery in rats following a single force C7 contusion injury (Onifer et al., 1997). Similarly, Schrimsher and Reier (Schrimsher and Reier, 1993) found that following a C4 laceration of the dorsal column, dorsolateral funiculus, or ventrolateral funiculus a similar pellet retrieval task was able to detect deficits only after DLF laceration without any notable recovery after 4 weeks. We believe that this report is the first record of a reaching task being able to discriminate between various grades of cervical white matter damage and the first to show significant recovery following injury. The modified pellet retrieval test also was more sensitive than the more commonly used footprint analysis. While the footprint analysis revealed $\mathrm{SPLA}_{2}$-III-induced forelimb dysfunction during locomotion, this assessment was largely unable to discriminate between injection groups and behavioral deficits were only observed in 2 of 5 measures. This finding emphasizes the need to perform multiple assessments when examining dysfunctions of the nervous system and evaluating repair strategies.

The greatest drawback of the modified pellet retrieval test is an inability to assess severe cervical SCI. In our device some of the $6 \mathrm{ng} \mathrm{sPLA}_{2}$-III-injected animals were not able to reach into the feeding tubes and perform the task. This could have been a result of lesion encroachment on the corticospinal tract (Fig. 15D) or undetected destruction of motor neurons and interneurons within the $\mathrm{C} 2 / 3$ gray matter. The latter is more likely since injections into the $\mathrm{C} 2 / 3$ interface would correspond with motor neurons controlling 
the acromiotrapezius and levitor claviculae, which would greatly compromise shoulder elevation (McKenna, 2000). Also, as stated above, only laceration of the dorsolateral funiculus and not the corticospinal tract effected the retrieval ability in the "Staircase Test" (Schrimsher and Reier, 1993). Following severe contusions the original "Staircase Test" may be more useful. However, it must be noted that the 6 ng animals showed some improvement at 4 weeks and the possibility of further improvements beyond 4 weeks could not be eliminated. While there was no expansion of the lesion area beyond the DLF in both 1.5 and $3 \mathrm{ng}$ injuries, there was a significant difference in behavioral measures. This could be explained by partial destruction of the rubrospinal tract in the 3 ng group with sparing of some fibers in the $1.5 \mathrm{ng}$ animals. While the lesions may appear homogenous, the loss of axons increased from the periphery to the center with the sparing of some axons seen in the center of the $1.5 \mathrm{ng}$ group.

In conclusion, injection of at least one type of sPLA $\mathrm{P}_{2}$ into the cervical white matter seems to have a bimodal effect on tissue and function. Initially normal tissue architecture is destroyed followed by the immigration of what is typically characterized as a beneficial cell type leading to remyelination. Similarly, our modified pellet retrieval device detected an initial dose-responsive dysfunction followed by recover between 2 and 3 weeks post injury. It is tempting to hypothesize what factors produced each facet of this bimodal response. With the knowledge that phospholipases are increased following CNS injury, current investigations in our lab are attempting to block the destructiveness of $\mathrm{sPLA}_{2}$ while harnessing the beneficial effects of increased Schwann cell immigration and remyelination. 


\section{CHAPTER IV}

\section{SPLA 2 INHIBITION PRIOR TO SPINAL CORD INJURY RESULTS IN FUNCTIONAL AND HISTOLOGICAL SPARING}

\section{INTRODUCTION}

In the previous chapters we have shown that many of the $\mathrm{SPLA}_{2}$ isoforms are present in the naïve spinal cord and within oligodendrocytes. We next showed that following spinal cord injury groups IIA and IIE increase by several fold and that sPLA $2_{-}^{-}$ IIA similarly increases within oligodendrocytes following two models of in vitro injury. We also showed that exogenously added recombinant human PLA $_{2}$-IIA is cytotoxic to both oligodendrocyte cultures and to naïve rodent spinal cords. Finally we demonstrated that blockade of $\mathrm{sPLA}_{2}$-IIA by pharmacological inhibition partially ameliorated the deleterious effects of TNF $\alpha$ and IL-1 $\beta$ or $\mathrm{H}_{2} \mathrm{O}_{2}$ in oligodendrocyte cultures. What remains to be seen is whether blockade of $\mathrm{sPLA}_{2}$ can create tissue and functional sparing following SCI in vivo.

Several inhibitors of sPLA $\mathrm{A}_{2}$ have shown in vivo efficacy in rodent models of neuropathology. Indoxam, a specific $\mathrm{SLA}_{2}$ inhibitor, was shown to offer protection against cerebral ischemia (Yagami et al., 2002b). Similarly, CDP-choline significantly restores phosphatidylcholine levels by differentially affecting $\mathrm{SPLA}_{2}$ and phosphocholine cytidylyltransferase after stroke (Adibhatla et al., 2006). Quinacrine / mepacrine, a non- 
specific inhibitor of $\mathrm{PLA}_{2}$ activity and anti-malarial drug, also showed sparing of hippocampal neurons (Phillis, 1996) and reduced infarct size following transient focal ischemia (Estevez and Phillis, 1997) and showed sparing of hippocampal neurons following kainite-injection (Ong et al., 2003). Finally membrane bound inhibitors of $\mathrm{SPLA}_{2}$ showed decreased inflammation in experimental autoimmune encephalomyelitis, a rodent model of multiple sclerosis (Pinto et al., 2003). Similarly, the small (487.63 MW) lipophilic molecule S3319 has been shown to be both a potent inhibitor of sPLA - -IIA with an $\mathrm{IC}_{50}=0.029 \mu \mathrm{m}$ (Church et al., 2001, Hansford et al., 2003) and orally active against infiltrating neutrophils following reperfusion injury in mice (Arumugam et al., 2003). However, this final compound has not been tested against CNS pathologies nor has any sPLA $\mathrm{A}_{2}$-IIA inhibitor ever been assessed following neurotrauma either in the brain or spinal cord.

The results presented here show that inhibition of $\mathrm{SPLA}_{2}$ activity prior to traumatic SCI results in increased oligodendrocyte survival, increased sparing of white matter, increased axons at the lesion epicenter. Accordingly, sPLA2 inhibition also decreased the lesion size and number of infiltrating cells migrating into the lesion. Finally, sPLA2 inhibition increased voluntary locomotion and accelerated recovery of voluntary bladder function.

\section{MARTERIALS AND METHODS}

Animals

A total of 20 female BALB/c mice (Charles River, Wilmington, MA) and 10 female 129/sv mice (Taconic, Germantown, NY) 6-8 weeks old, were used in this study. 
All surgical interventions and postoperative animal care were performed in accordance with the Guide for the Care and Use of Laboratory Animals (National Research Council, National Academy Press, Washington, D.C., 1996) and the Guidelines of the University of Louisville Institutional Animal Care and Use Committee.

Experimental groups and dosing

It has been previously determined that certain mouse strains have a natural disruption of the sPLA 2 -IIA gene (e.g. 129/sv) (Kennedy et al., 1995). Mice were divided into 5 groups (see Table 5) based on sPLA 2 -IIA expression, the state of spinal cord injury, and pharmacological inhibition of SPLA $_{2}$-IIA. Beginning two days prior to surgery and for 2 weeks afterwards animals were give either S3319 (10 mg/kg i.p.; Sigma-Aldrich, St. Louis, MO), a potent sPLA 2 -IIA enzymatic inhibitor (Hansford et al., 2003), or vehicle (70\% DMSO) mixed fresh daily. The dose was reduced to $5 \mathrm{mg} / \mathrm{kg}$ during the second week post injury. This dose was selected based on previous in vivo efficacy and physiologically activity (Arumugam et al., 2003). 
Table 5. Experimental Groups

\begin{tabular}{cccccc}
\hline Group \# & Strain & sPLA $_{2}$-IIA gene & Injection & SCI & $\mathrm{n}$ \\
\hline 1 & BALB/c & $+/+$ & S3319 & $0.45 \mathrm{~mm}$ & 8 \\
2 & BALB/c & $+/+$ & Veh & $0.45 \mathrm{~mm}$ & 8 \\
3 & BALB/c & $+/+$ & Veh & Sham & 4 \\
4 & $129 / \mathrm{sv}$ & $-/-$ & Veh & $0.45 \mathrm{~mm}$ & 8 \\
5 & $129 / \mathrm{sv}$ & $-/-$ & Veh & Sham & 4 \\
\hline
\end{tabular}

Contusive spinal cord injury

Mice were injected with ketamine and xylazine $(90+10 \mathrm{mg} / \mathrm{kg}$, i.p., Lloyd Laboratories, Shenandoah, IA), $1 \mathrm{ml}$ of normal saline (s.c.), and placed on a homeothermic blanket. The back was shaved and skin was cleansed with betadine. Skin was incised and the underlying muscles were dissected to expose the dorsal halves of the T7-T13 vertebrae. The exposed vertebral column was stabilized using bilateral transverse supports placed underneath the spinal column which were developed at the University of Louisville (Zhang et al., 2004, Cao et al., 2005, Onifer et al., 2005, Iannotti et al., 2006, Liu et al., 2007, Onifer et al., 2007). A dorsal laminectomy was performed at the T8-11 level to expose the spinal cord and bleeding was controlled. The body was immobilized with a horizontal brace gently secured over the middle of the back to prevent it from moving upward during the SCI. Rats received either a $0.45 \mathrm{~mm}$ displacement injury between the $9^{\text {th }}$ and $10^{\text {th }}$ thoracic vertebra, inflicted via the Louisville Imapct System Aparatus (LISA) or sham laminectomy as previously published by our 
center. After the injury, displacements were recorded and compared to insure lesion uniformity. Post-operative care followed those described previously (Iannotti et al., 2004)

Behavioral assessments

During the week prior to baseline recordings mice were gentled and then acclimated to all devices. Voluntary over ground locomotion was assessed by the Basso Mouse Scale for locomotion (BMS) as previously described (Basso et al., 2006). The test was performed by two blinded scorers on day 3 and weeks 1 through 9 post surgery.

As is common following severe spinal cord injury, bladder function was lost in all injured animals for at least one day following injury. Bladders were manually expressed twice daily and the volume of expelled urine recorded. When expressing bladders, an experienced handler gave each mouse a bladder score determined by size and outflow. A void bladder received a score of 0, small $=1$, medium $=2$, large $=3$, and very large $=4$. Scores were summed over 48 hrs to eliminate feeding variability.

Tissue preparation

After week nine assessment all mice were given a lethal dose of ketamine (180 $\mathrm{mg} / \mathrm{kg})$ and xylazine $(20 \mathrm{mg} / \mathrm{kg})$ and then transcardially perfused with $5 \mathrm{ml}$ of $4{ }^{\circ} \mathrm{C}$ PBS followed by $20 \mathrm{ml} 4 \%$ PFA. A $2 \mathrm{~cm}$ section of spinal cord containing the injury epicenter was removed and samples were post fixed for $3 \mathrm{hrs}$ in $4 \%$ PFA and transferred to $30 \%$ sucrose for 3 days. The spinal cords were then embedded in tissue freezing 
medium and sliced transversly at $40 \mu \mathrm{M}$ to create eight consecutive sets with sections in each set separated by $320 \mu \mathrm{m}$.

Luxol fast blue staining

To determine the area of spared white matter, one complete set of slides was stained with luxol fast blue for myelin as has been previously reported (Liu et al., 2006, Titsworth et al., 2007). Briefly, sections were dehydrated through 95\% ethanol and then submerged in Luxol Fast blue solution (0.1\% luxol fast blue, 0.05\% acetic acid, in 95\% ethanol) at $65^{\circ} \mathrm{C}$ for $2 \mathrm{hr}$. Slides were then differentiated in $0.05 \%$ lithium carbonate solution and $70 \%$ ethanol. Sections were dehydrated through graded ethanol, rinsed with xylene, and mounted.

\section{Cresyl Echt Violet-Eosin staining}

A second set was stained as previously reported (Liu et al., 2006). Briefly, sections were stained with $0.5 \%$ cresyl echt violet (Sigma, St. Louis) in $20 \%$ ethanol for 8 min followed by eosin solution ( $25 \%$ eosin, $70 \%$ ethanol, $0.5 \%$ acetic acid) for $20 \mathrm{sec}$. Sections were then dehydrated, rinsed with xylene, and mounted.

Immunofluorescence double labeling Immunofluorescence double labeling at the injury epicenter was performed on different tissue sets using previously described methodologies (Liu et al., 2004b). In brief, various combinations of antibodies were used (see Table 6). The paired antibody solutions were applied to the sections overnight at $4^{\circ} \mathrm{C}$. The following day, sections were 
incubated with fluorescein-conjugated goat anti-mouse (1:100; Jackson Immunoresearch, West Grove, PA) and Texas red-conjugated goat anti-rabbit (1:100; Jackson Immunoresearch) antibodies. Primary antibody omission controls and normal mouse, rabbit, and goat serum controls were used to further confirm the specificity of the immunofluorescence double labeling. Images were taken using a Nikon Eclipse 90i confocal microscopy (Nikon Instruments; Melville, NY).

Table 6. Antibodies Used

\begin{tabular}{ccccc}
\hline Antibody Clone & Source & Working Dilution & Vendor/lot No. & Reference \\
\hline anti-sPLA 2 -IIA & Rabbit & $1: 100$ & Cayman Chemical & (Moses et al., 2006) \\
anti-SMI-31 & Mouse & $1: 1000$ & Covance & (Liu et al., 2006) \\
anti-GFAP & Rabbit & $1: 300$ & Sigma & (Liu et al., 2006) \\
anit-Olig1 & Mouse & $1: 200$ & Millipore & (Arnett et al., 2004) \\
\hline
\end{tabular}

Volume calculations

Three-dimensional (3-D) reconstruction and unbiased estimation of tissue volumes were calculated using the Cavalieri method (Michel and Cruz-Orive, 1988) on an Olympus BX60 microscope (Olympus America, Inc., Mellville, NY) equipped with a Neurolucida system (MicroBrightField, Colchester, VT). Areas were calculated by drawing contours to define regions of interest on each section. Individual subvolumes of spared tissue were calculated by multiplying the cross-sectional area $A \times d$, where $d$ represents the distance between sections (either $320 \mu \mathrm{m}$ or $960 \mu \mathrm{m}$ ). The volume of total 
of tissue was calculated by summing their individual subvolumes of each section measured.

In luxol fast blue stained sections the area of spared white matter in each section was determined by subtracting the unstained region of each spinal cord section from the total spinal cord area. The percentage of spared tissue was calculated by dividing the total volume of spared white matter in experimental animals to that on controls (Oorschot, 1994). Similarly, the lesion volume was defined as the GFAP negative area within the lesion core. The lesion epicenter for each animal was defined as the spinal section in each animal with the least amount of spared myelin or the greatest percentage of GFAP negative area.

Stereology

Unbiased stereological counts were made using the probes in Stereo Investigator (MicroBrightField, Colchester, VT). SMI-31 positive axons passing through the lesion epicenter were counted as well as oligodendrocytes and infiltrating nuclei within a $4 \mathrm{~mm}$ section of cord centered on the lesion epicenter (Table 7). In brief, a systematic random sampling grid was overlaid on a traced spinal section yielding approximately 5 - 20 sampling sites per section. Next, profiles were counted within either an unbiased counting square or cube under $100 \times$ oil immersed optical magnification. In the optical fractionator probe the height was $20 \mu \mathrm{m}$ with at least a $5 \%$ guard zone. The resulting Schmitz-Hof Coefficient of Error was $<0.1$ with an average of 212 profiles counted per animal. 
Table 7. Stereology Parameters

\begin{tabular}{ccccc}
\hline Object of interest & Stain & Probe & SRS grid $(\mu \mathrm{m})$ & Dissector $(\mu \mathrm{m})$ \\
& & & & \\
\hline \multirow{2}{*}{ Axons } & SMI-31 IF & Fractionator & Cnt $=305 \times 274$ & $15 \times 15$ \\
& & Exp $=195 \times 162$ & $15 \times 15$ \\
\cline { 3 - 4 } Oligodendrocytes & Olig-1 IF & Optical & Cnt $=325 \times 330$ & $60 \times 60$ \\
& & Fractionator & Exp $=226 \times 195$ & $95 \times 95$ \\
\cline { 3 - 4 } Inflilt. Nuclei & Cresyl Eosin & Optical & Cnt $=305 \times 274$ & $60 \times 60$ \\
& & Fractionator & Exp $=195 \times 162$ & $15 \times 15$ \\
& & & & \\
\hline
\end{tabular}

Statistical Analysis

One-way analysis of variance (ANOVA) with post hoc Tukey's HSD was used to determine statistical significance of three or more groups. A multiple analysis of variance (MANOVA) with post hoc Tukey's HSD was used to determine statistical significance of three or more groups when repeated measures were taken from each animal overtime or space. A $p<0.05$ was considered statistically significant.

\section{RESULTS}

Inhibition of $\mathrm{SPLA}_{2}$ Increases Behavioral Recovery Following SCI.

Overgrown locomotion as assessed by the BMS showed a significant difference among experimental groups (MANOVA $\mathrm{F}_{4,349}=1769, p<0.0001$ ) and over time (MANOVA $F_{10,349}=113, p<0.0001$; Fig. 20). Post hoc analysis revealed that $129 \mathrm{svj}$ mice receiving a spinal cord injury ( $\mathrm{sPLA}_{2}-/-\mathrm{SCI}$ ) did significantly better than their wild type counterparts BALB/c (Veh SCI) at week $5(\mathrm{p}<0.05)$, week $7(\mathrm{p}<0.001)$, week 8 $(p<0.001)$, and week $9(p<0.05)$. Similarly, inhibition of sPLA $\mathrm{A}_{2}$ with S3319 in contused $\mathrm{BALB} / \mathrm{c}$ mice resulted in significantly improved BMS scores over vehicle controls at 
week $7(p<0.05)$, week $8(p<0.001)$, and week $9(p<0.01)$. At week 9 the BMS scores for $\mathrm{sPLA}_{2}-/-$ and S3319 treated mice receiving a spinal cord injury was $2.58 \pm 0.51$ and $2.66 \pm 0.78$ respectively, while among vehicle control mice receiving a spinal cord injury the week 9 score was $1.43 \pm 0.85$. Within the BMS a score of 1 given for slight ankle movement, a score of 2 correlates to extensive ankle movement, and a score of 3 corresponds to plantar placing of the paw or dorsal stepping. At week 9 all animals within the S3319 group scored were able to plantar place at least one paw except for one animal. While only two animals in the vehicle treated control plantar place at least one paw. Interestingly the $\mathrm{sPLA}_{2}-/$ - mice were each able to place one paw, with the exception of one who could place both. However, their stepping ability seemed relatively intact indicating that their low scores were more a function of severe scoliosis that developed in this group resulting in a defined foot preference.

Bladder dysfunction has consistently been ranked as one of the top concerns among paraplegics and quadriplegics, usually of higher importance than the loss of locomotion (Benevento and Sipski, 2002, Anderson, 2004). In this study bladder function following SCI also showed significant difference among experimental groups $\left(\right.$ MANOVA F $\left._{4,388}=97.74, p<0.0001\right)$ and over time $\left(\right.$ MANOVA $F_{15,388}=8.095$ $p<0.0001$; Fig. 20). Post hoc analysis revealed that contused 129 svj (sPLA $2^{-/-}$SCI) mice had a significantly lower bladder volume than untreated BALB/c mice expressing $\mathrm{SPLA}_{2}-$ IIA (Veh SCI), between days $3(p<0.001)$ and day $25(\mathrm{p}<0.001)$. Similarly, pharmacological inhibition of sPLA 2 by S3319 resulted in significantly lower bladder volumes at days $19(\mathrm{p}<0.05)$ through $25(\mathrm{p}<0.001)$ when compared to vehicle control $\mathrm{BALB} / \mathrm{c}$ mice receiving contusion. Stated differently, the bladder volumes of contused 
$129 \mathrm{svj} \mathrm{(-/-)} \mathrm{mice} \mathrm{became} \mathrm{statistically} \mathrm{similar} \mathrm{to} \mathrm{sham} \mathrm{animals} \mathrm{at} \mathrm{day} 7$ post injury ( $>>0.05)$. With S3319 the bladder function returned on day 13. However, bladder function in the vehicle controls did not mirror sham animals until day 31 . This suggests that both the pharmacological and genetic manipulation of $\mathrm{sPLA}_{2}$ following severe SCI resulted in a return of bladder function between two and three weeks earlier than controls. Since this experiment utilized a thoracic lesion, far above the sacral micturition center (S2-4), any improvement suggests an increased signaling from the higher centers passing through the lesion epicenter. 
Figure 20. sPLA $A_{2}$ Inhibition Increases Functional Recovery Following Severe Spinal Cord Injury.

In the top graph sPLA 2 inhibition by i.p. injection with $\mathrm{S} 3319$ prior to injury results in significantly improved voluntary over ground locomotion at weeks 7,8 , and $9(+-+++)$. Similarly, a frame shift mutation in the $\mathrm{SPLA}_{2}$-IIA gene ( $\left.\mathrm{SPLA}_{2}-/-\right)$ results in statistically significant increases in locomotion at weeks $5,7,8$, and $9\left(*_{-} * * *\right)$. The bottom graph shows the average voiding volume of urine. Mice lacking the $\mathrm{SPA}_{2}$ gene returned to baseline volumes on day 7. Mice injected with S3319 returned to baseline by day 13 . While vehicle control mice only returned to baseline on day 31. Graphs are presented as $\bar{x} \pm \mathrm{SE}$. Between comparisons of injured animals at a single time point, "+ or $* "=$ $\mathrm{p}<0.05, “++$ or $* * "=\mathrm{p}<0.01, “+++$ or $* * * ”=\mathrm{p}<0.001$. 
A

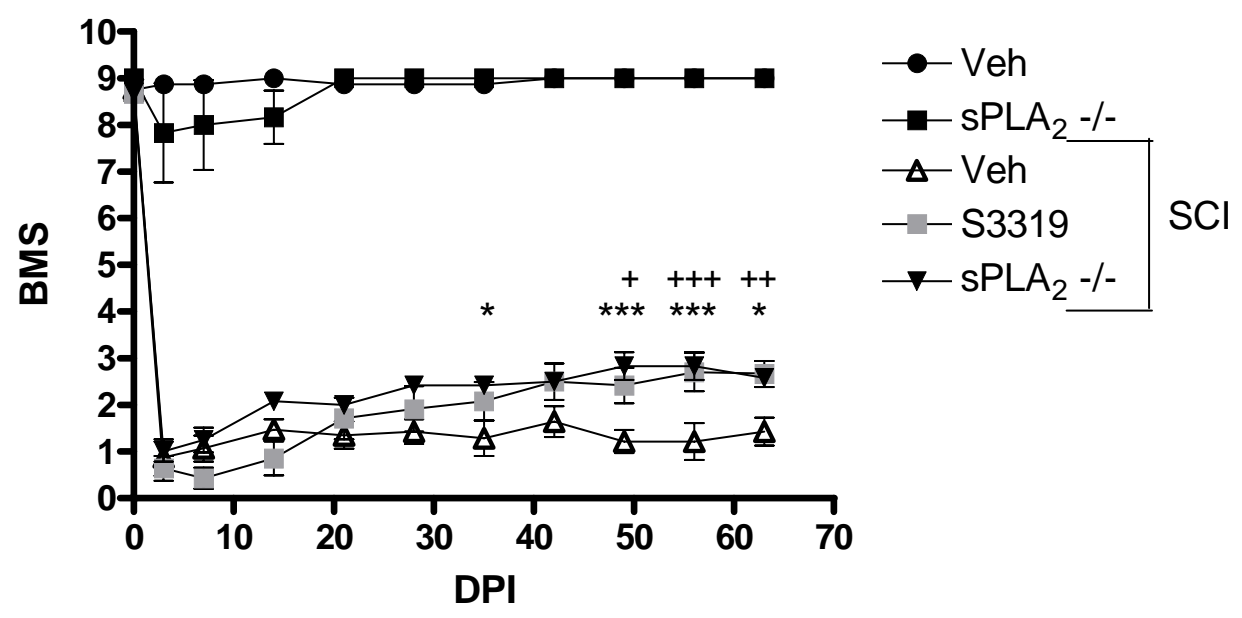

B

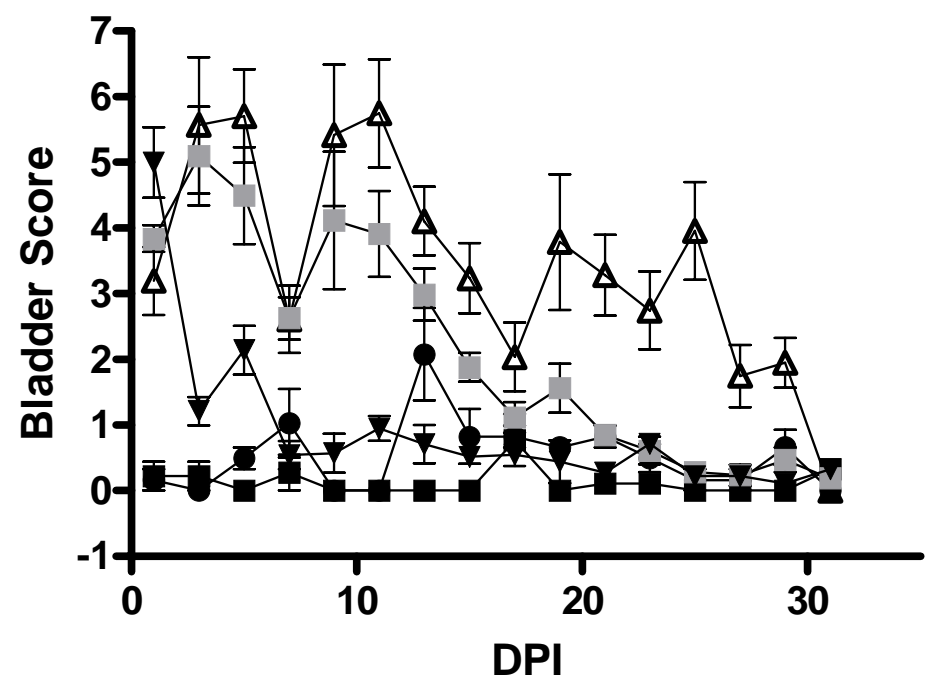

$\rightarrow-$ Veh
$\rightarrow-$ sPLA2 -/-
$\triangle-$ Veh
$--S 3319$
$\rightarrow$ SPLA 2 -/- 
Inhibition of sPLA 2 Increases Histological Recovery Following SCI.

To confirm the observed behavioral improvements have a histological correlate, mice were sacrificed and tissue was sectioned. First luxol fast blue and cresyl eosin staining was performed as a general measure of white matter sparing and inflammation respectively. At 9 weeks post injury, significant differences existed among groups with respect to total volume of shared myelin within an $16 \mathrm{~mm}$ section of cord centering on the epicenter (Fig. 21 top right; ANOVA F $4,35=91.44, p<0.0001$ ). Both the 129 svj mice lacking sPLA 2 expression $\left(\mathrm{sPLA}_{2}-/-\mathrm{SCI} ; \mathrm{p}<0.001\right)$ and the $\mathrm{S} 3319$ treated $\mathrm{BALB} / \mathrm{c}$ mice $(\mathrm{S} 3319 \mathrm{SCI} ; \mathrm{p}<0.05)$ showed significantly more spared myelinated tissue than the vehicle treated BALB/c mice. Furthermore the area of spared myelin was calculated at each 0.96 $\mathrm{mm}$ interval rostral and caudal from the lesion (Fig. 21 bottom right). Again this showed significant differences among the injury groups (MANOVA $\left.\mathrm{F}_{4,595}=488.7, p<0.0001\right)$ and among distances from epicenter (MANOVA $\left.F_{16,595}=116.8, p<0.0001\right)$. Post hoc analysis revealed that $129 \mathrm{svj}$ mice $\left(\mathrm{sPLA}_{2}-/-\mathrm{SCI}\right)$ were significantly different from vehicle treated $\mathrm{BALB} / \mathrm{c}$ mice (Veh $\mathrm{SCI}$ ) at all points rostral (varying significance from $\mathrm{p}<0.001$ to $\mathrm{p}<0.05)$ and at $-0.96 \mathrm{~mm}$ caudal $(\mathrm{p}<0.001)$ and $-1.92 \mathrm{~mm}$ caudal $(\mathrm{p}<0.01)$ to the lesion epicenter. In contrast differences in the sparing of myelinated tissue was only seen between S3319 treated BALB/c mice (S3319 SCI) and vehicle treated controls (Veh $\mathrm{SCI})$ at $1.92 \mathrm{~mm}(\mathrm{p}<0.05)$ and $0.96 \mathrm{~mm}(\mathrm{p}<0.001)$ rostral to the injury epicenter. Sparing of white matter rostral to the lesion epicenter has also been seen in other treatments for spinal cord injury such as bFGF (Rabchevsky et al., 1999, Rabchevsky et al., 2000) and implantation of FGF2 secreting cells (Zai et al., 2005). 
Interesting pronounced demyelination was noted to extend rostrally much further in the dorsal columns of the untreated animals (Fig. 21 graph bottom right). Pictured is demyelination of the dorsal column $10 \mathrm{~mm}$ rostral to the lesion epicenter (Fig. 21 arrow in right composite). Similarly differences in white matter sparing were observed in various mice strains following SCI and was attributed to longer lesion extension observed in the dorsal columns (Kigerl et al., 2006). Not surprisingly the areas of demyelination correlated strongly with those areas expressing increased sPLA 2 -IIA at 9 weeks (Fig. 22). 


\section{Figure 21. sPLA $A_{2}$ Inhibition Increases Myelin Sparing}

A composite (A) of a representative animal from each group showing consecutive luxol fast blue stained sections at epicenter and various distances rostral to the epicenter. Note the greater spared myelin in the S3319 treated and sPLA $2^{-/-}$mice as compared to the vehicle control with SCI. Note that demyelination of the dorsal columns extends rostrally at least $10 \mathrm{~mm}$ (arrow). Quantification of the total volume of spared white matter (B) in a $16 \mathrm{~mm}$ section of spinal cord centering on the epicenter and a graph of the subvolumes (C) confirm the observed demyelination. Note the decreased sparing of white matter present in the rostral but not the caudal direction. $\mathrm{sPLA}_{2}-/-$ mice show significant white matter sparing at all points rostral to the epicenter measured $(\mathrm{C} ;+)$ while mice injected with S3319 only show increased sparing at $0.96 \mathrm{~mm}$ and $1.92 \mathrm{~mm}$ rostral to the epicenter $(*)$. 
A
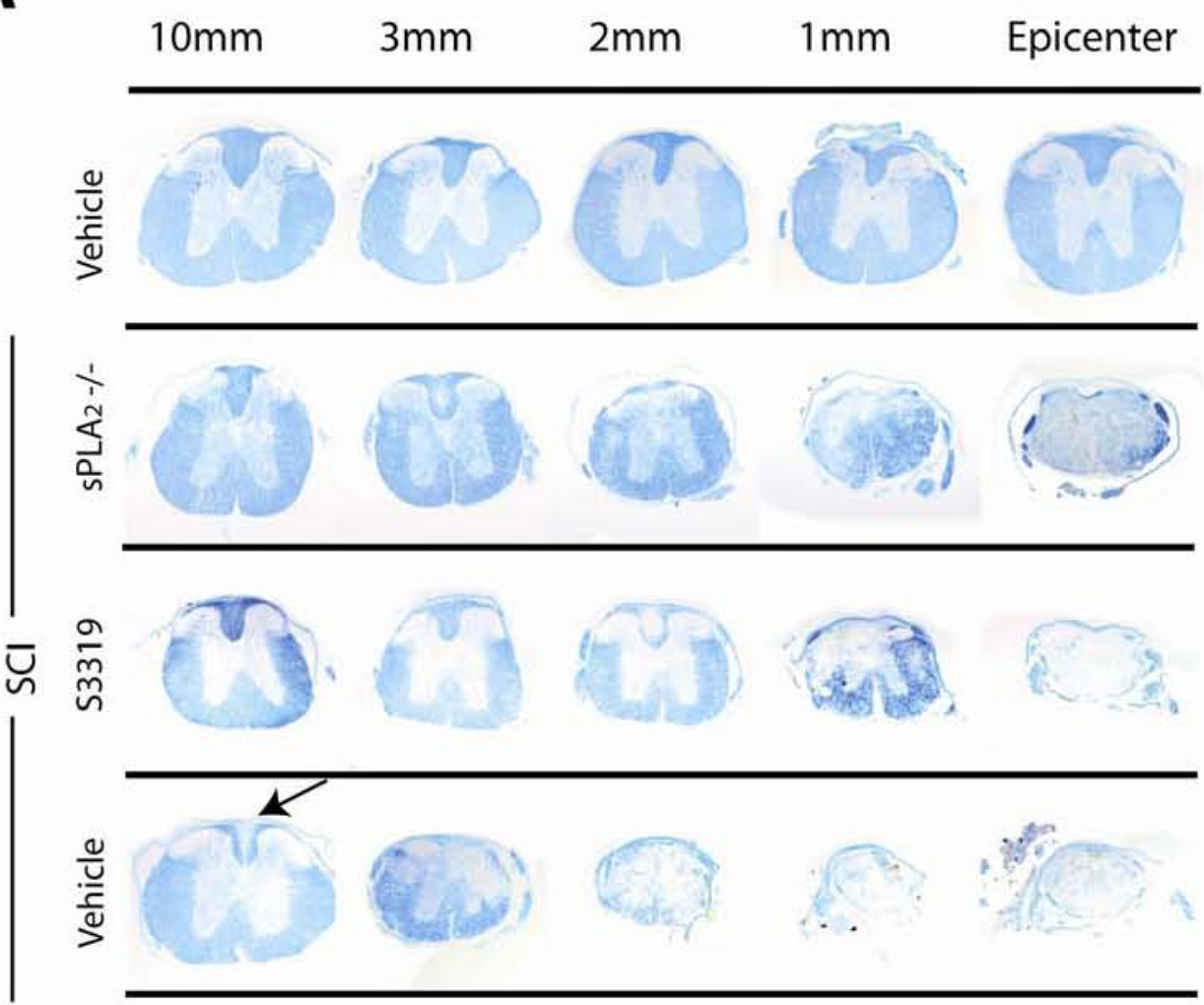

B

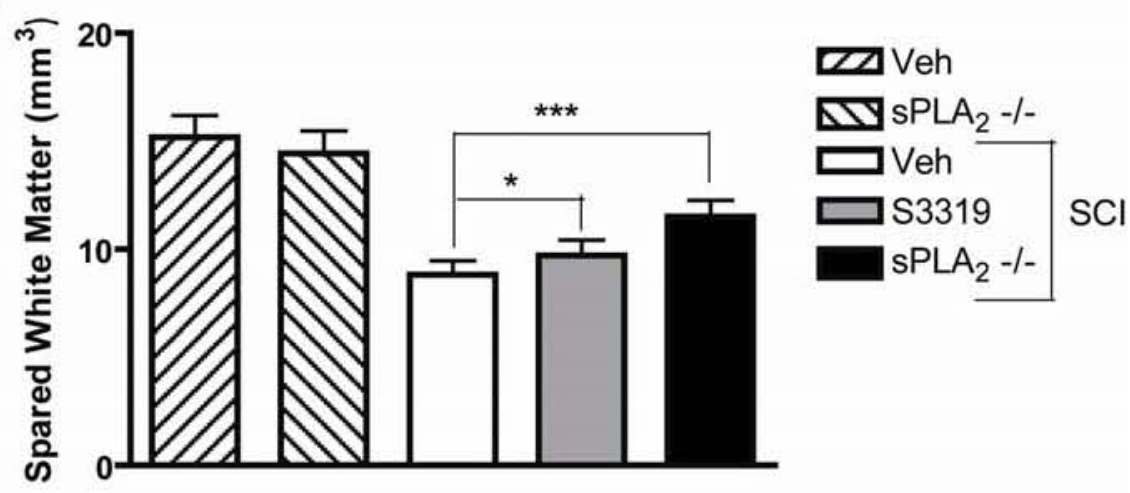

C

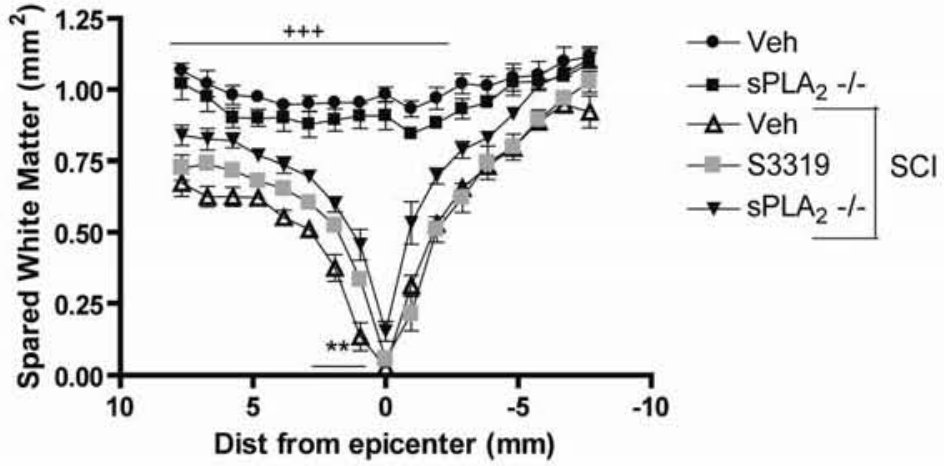



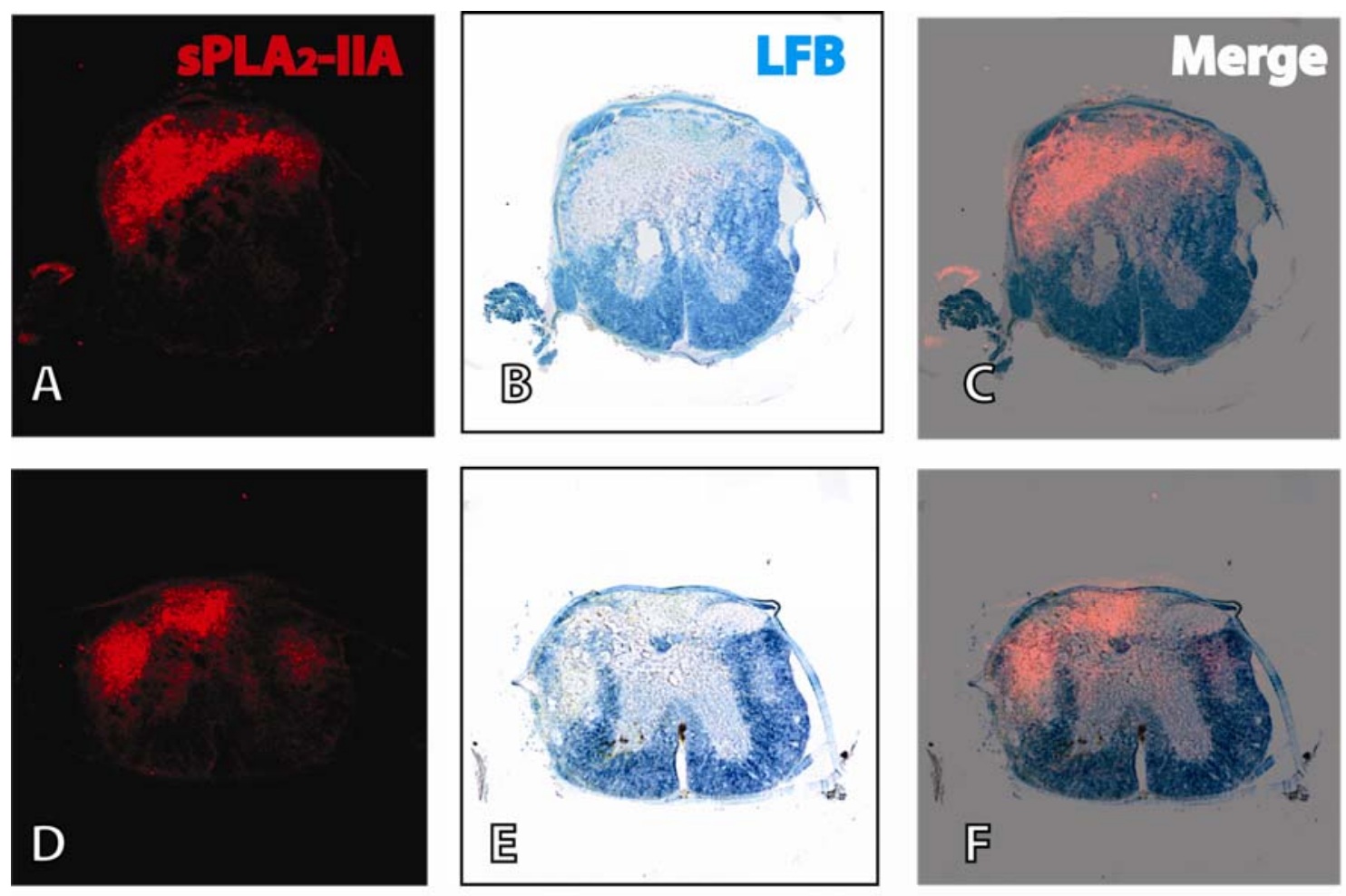

Figure 22. sPLA

Sections from different tissue sets stained for either sPLA2-IIA immunoreactivity (A \&

D) or luxol fast blue stain for myelin (B \& E). Images are from two different S3319

animals either $1.6 \mathrm{~mm}$ rostral (A-C) or $1.28 \mathrm{~mm}$ rostral (D-F) to the lesion epicenter.

Note that in D-F the three distinct areas of demyelination in the dorsal columns and both lateral funiculi correlated with that of demyelination with very little immunoreactivity in the dorsal horns. 
Following spinal cord injury there is a migration of millions of small infiltrating cells into the lesion area that are easily seen in CE stained sections. The infiltration of inflammatory cells whose nuclei is dark blue corresponds nicely with the areas of demyelination seen in luxol fast blue stained sections (Compare Fig 21A \& Fig 23A). Also similar to a LFB stained section, increased inflammation is again seen in the dorsal columns rostral to the lesion epicenter (arrow in Fig 23A \& B). Stereological quantification of these cell's nuclei allow for a rough approximation of how different conditions affect inflammation. At 9 weeks post injury, significant differences existed among groups with respect to number of infiltrating nuclei within a $4 \mathrm{~mm}$ section of spinal cord centering on the epicenter (Fig. 23C; ANOVA F $_{4,25}=137.8, p<0.0001$ ). There was virtually no infiltrating nuclei seen in either of the sham conditions however $1.25 \times 10^{6} \pm 203,000$ cells were counted in the vehicle control mice while only $0.82 \times 10^{6} \pm$ $157,000$ cells (S3319; $\mathrm{p}<0.001)$ and $0.93 \times 10^{6} \pm 70,000$ cells ( $\left.\mathrm{sPLA}_{2}-/-; \mathrm{p}<0.001\right)$ were seen in the treated conditions. This signifies a $34 \%$ and $25 \%$ decrease inflammation in the S3319 and $\mathrm{sPLA}_{2} /$ - groups respectively. Suggesting that as was seen in other in vivo injury models, that S3319 has an anti-infiltrating effect that carries over to neuroinflammation. 


\section{Figure 23. sPLA $A_{2}$ Inhibition Results in Decreased Inflammation Following SCI}

A composite (A) of the same representative animal from each group that is shown in Fig. 21 , showing sections at epicenter and various distances rostral to the epicenter stained with cresyl eosin stain. Note the decreased infiltration of infiltrating cells in the S3319 and $\mathrm{SPLA}_{2}-/-$ mice as compared to the vehicle control with SCI. Note that similar to the demyelination, inflammation extends $10 \mathrm{~mm}$ rostrally in the dorsal columns of the vehicle control animal (arrow). 3-D reconstructions (B) of the same cords in A. Quantification of the infiltrating cell nuclei $(\mathrm{C})$ in the spinal cord centering on the epicenter shows that inhibition of $\mathrm{SPLA}_{2}$ results in decreased infiltration of small infiltrating nuclei. 

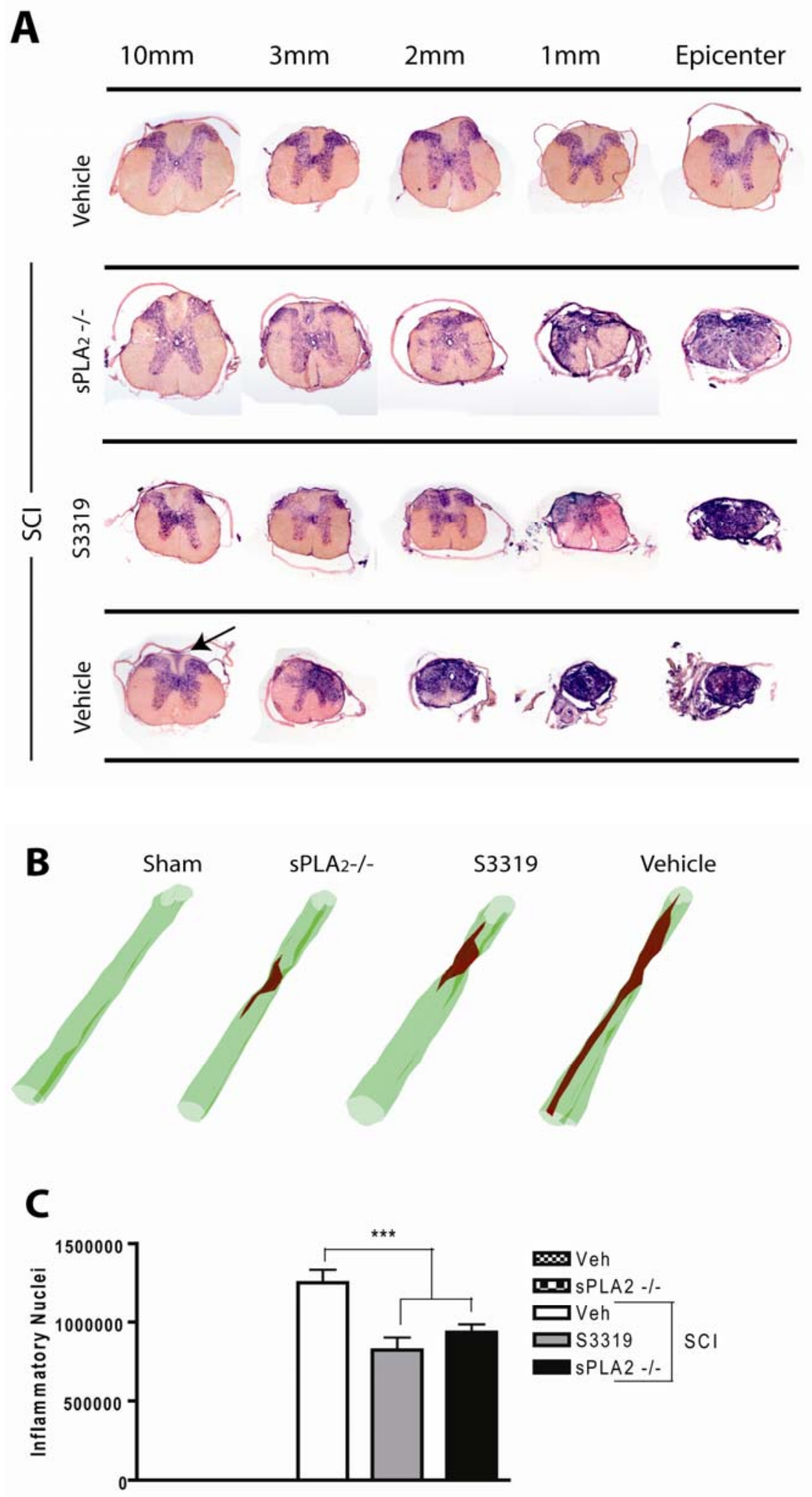
To measure the volume of a lesion we used GFAP stained sections and measured the area within the reactive glial border which was the spinal cord lesion epicenter. The GFAP $^{-}$lesion area was quantified at $320 \mu \mathrm{m}$ intervals as a percentage of total spinal cord area. Again, there was significant differences existed among groups (Fig. 25A-C \& J; MANOVA $\left.F_{2,273}=43.09, p<0.0001\right)$ and with respect to distance from the lesion epicenter (MANOVA $\left.F_{12,273}=86.37, p<0.0001\right)$. Mice lacking sPLA $_{2}$-IIA expression showed significantly smaller lesions at $1.28 \mathrm{~mm}(\mathrm{p}<0.001), 0.96 \mathrm{~mm}(\mathrm{p}<0.001), 0.64 \mathrm{~mm}$ $(\mathrm{p}<0.001), 0.32 \mathrm{~mm}$ rostral $(\mathrm{p}<0.001)$ and at $0.32 \mathrm{~mm}$ caudal $(\mathrm{p}<0.05)$ to the lesion. Similarly, pharmacological inhibition of sPLA 2 -IIA by S3319 resulted in significant sparing rostral to lesion at $1.28 \mathrm{~mm}(\mathrm{p}<0.001)$ and $0.96 \mathrm{~mm}(\mathrm{p}<0.001)$. It is interesting to note that once again there appears to be a preferential sparing of tissue rostral but not caudal to the lesion epicenter. Quantification and analysis of tissue area showing astrogliosis yielded virtually identical significance.

However the decrease in lesion size and white matter sparing is superfluous in a thoracic lesion if the number axons traversing the lesion epicenter is unaltered. Therefore, the number of axons found in the lesion epicenter was quantified using unbiased stereology with a fractionator probe revealing a significant difference among injury groups at 9 wks post injury (Fig. 25G-I \& K; ANOVA $F_{2,15}=6.198$, p $<0.01$ ). Almost double the number of axon profiles were counted at the lesion epicenter in both Balb/c mice treated with the $\mathrm{SPLA}_{2}$ inhibitor $\mathrm{S} 3319(\bar{x}=11,285 \pm 2880)$ and 129 svj mice lacking sPLA 2 -IIA expression (sPLA $2-/-\mathrm{SCI} ; \bar{x}=11,450 \pm 3817$ ) when compared to vehicle controls receiving spinal cord injury $(\bar{x}=6,206 \pm 1713)(\mathrm{p}<0.05)$. However 
neither of the treated animals achieved $10 \%$ of axon counts in the uninjured control animals $(\mathrm{Balb} / \mathrm{c}=121,830 \pm 1,053 ; 129 \mathrm{svj}=128,039 \pm 7,398)$. 


\section{Figure 24. sPLA $A_{2}$ Effect on Glial Lesion and Axons Within the Epicenter}

A-C) Low power images taken from $960 \mu \mathrm{m}$ rostral from the lesion epicenter showing decrease in lesion size after $\mathrm{SPLA}_{2}$ inhibition. D-F) low power images of the lesion epicenter showing no difference in lesion size. White boxes indicate areas from which the high power images G-L were taken. Images G-I show double labeling of GFAP (red) and SMI-31 (green) at the lesion epicenter. J-L) Single labeled images showing an increase in SMI-31 labeled axons in both the S3319 group $(\mathrm{K})$ and the sPLA $2_{2} /-$ animals (L). M) Quantification of GFAP negative lesion area over a $4 \mathrm{~mm}$ section centered on the epicenter. Note the significantly smaller lesion size between $\mathrm{sPLA}_{2}-/-$ mice at 1.28 $\mathrm{mm}, 0.96 \mathrm{~mm}, 0.64 \mathrm{~mm}$, and $0.32 \mathrm{~mm}$ rostral as well as $0.32 \mathrm{~mm}$ caudal to the lesion. Similarly, mice injected with S3319 show smaller lesion size rostral to lesion at $1.28 \mathrm{~mm}$ and $0.96 \mathrm{~mm}$. N) Quantification of the axon profiles at the lesion epicenter revealed almost twice as many spared axons at the lesion epicenter in both treated condition than in the vehicle control $(\mathrm{p}<0.05)$. Scale bar $=300 \mathrm{~mm} \mathrm{A-B;} 200 \mathrm{~mm} \mathrm{C-D;} 50 \mathrm{~mm}$ E-H. $\mathrm{p}<0.05 *, \mathrm{p}<0.001 * * *$ 

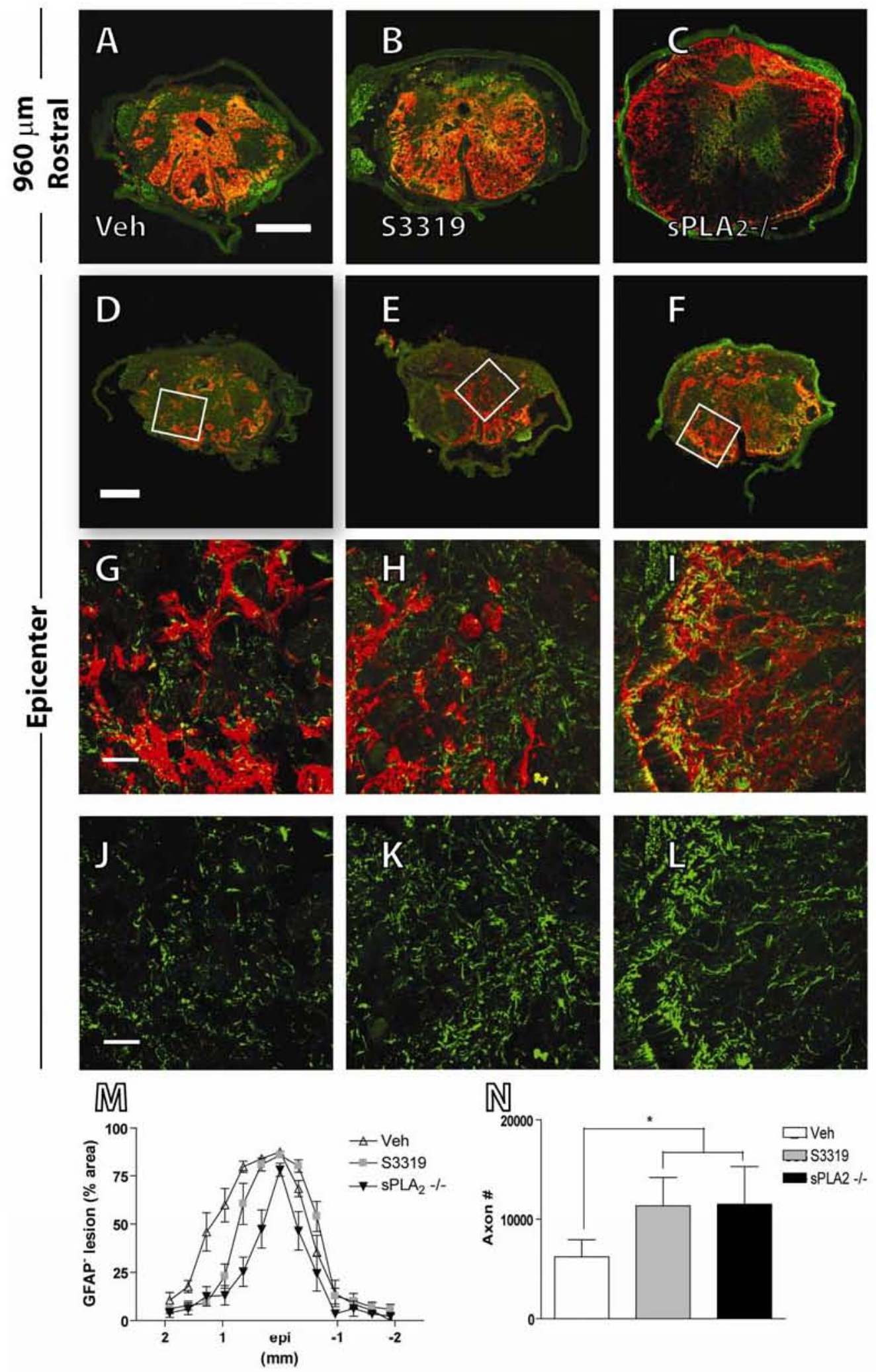
Since there seemed to is preservation of Luxol fast blue staining in the rostral dorsal columns in both the S3319 treated and 129svj mice (arrow in Fig. 21), we next need to determine if axons were present in these areas. Three consecutive sections, $8 \mathrm{~mm}$ rostral to the lesion epicenter, were stained in each animal. The first was LFB strained for myelin (Fig. 25 A-C), the second was CE stained (Fig. 25 D-F), and the third was GFAP/SMI-31/Hoechst triple labeling (Fig. 25 G-U). First, this comparison revealed that the lesion area took up less of the dorsal column in both the S3319 and 129svj groups. Secondly, axons were present both the S3319 treated and 129svj animals (Fig. 25 Q, R, T, \& U), when the lesion was defined as the area with increased gliosis. Suggesting that treatment with S3319 resulted in at least partial sparing of both white matter and axons rostral to the lesion epicenter. However, the LFB sapring appeared to be much greater, suggesting that some axons were lost while their myelin sheaths were spared. An alternative hypothesis that has been proposed by some authors is that all axons were initially destroyed and what is seen are regenerating fibers though spared myelin sheaths (Raisman, 2004). 
Figure 25. Dorsal Column Injury

A-F are from different sections stained with luxol fast blue (A-C) and crestyl eosin (D-F).

G-R are triple immuno-labeled with anti-SMI-31 (green), anti-GFAP (red), and hoechst (blue), All sections are from $8 \mathrm{~mm}$ rostral to injury epicenter. Scale bar in $\mathrm{R}=100 \mu \mathrm{m}$. 

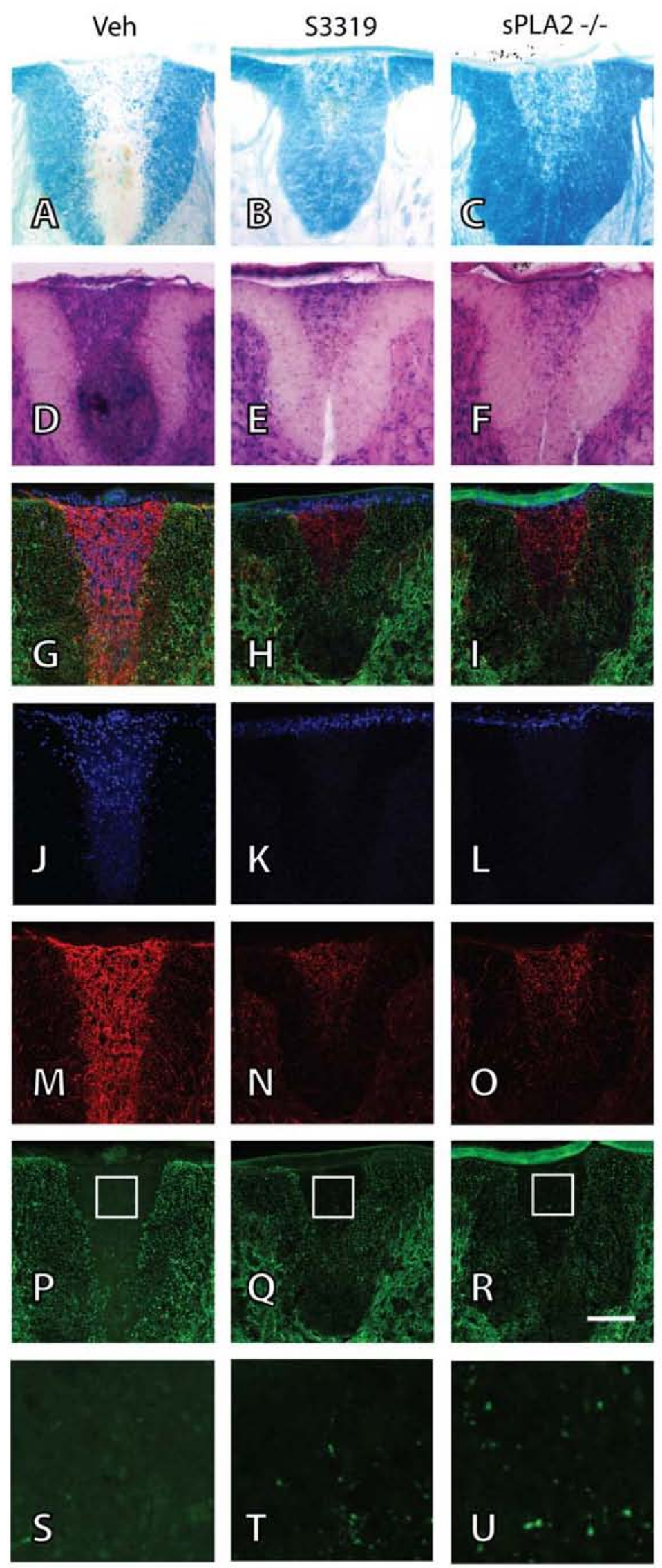
In previous work we noted that injections of $\mathrm{SPLA}_{2}$ at the grey and white matter interface had a greater impact on white matter injury. Additionally, we showed that inhibition of sPLA $A_{2}$ by S3319 was able to partially protect differentiated oligodendrocyte precursor cells in vitro in two injury models. Therefore we first used immunohistochemistry to confirm that $\mathrm{SLA}_{2}$-IIA was in fact present in oligodendrocytes (Fig. 26A-C). We next used unbiased stereological measurement to count the number of spared oligodendrocytes within a $2 \mathrm{~mm}$ section of spinal cord centering on the lesion center. Since Olig1+ stains the entire cell body sections were counter stained with Hoechst to prevent over counting. There was a significant difference in the number of oligodendrocytes at 9 weeks post injury (ANOVA $F_{2,15}=17.67$, p $<0.0001$; Fig. 26D). Post hoc analysis revealed that among the injured animals, $\mathrm{S} 3319(\bar{x}=14,200 \pm 3,536)$ and sPLA $_{2}-/(\bar{x}=24,200 \pm 7,181)$ animals had a significantly higher number of Olig1+ oligodendrocytes than vehicle controls $(\bar{x}=6,360 \pm 4,179)$. This suggests that as was seen in vitro, inhibition of $\mathrm{SPLA}_{2}$ could result in partial sparing of oligodendrocytes following injury. However, it remains to be seen if this results from prevention of apoptosis or rather a decrease in inflammation resulting in a more permissive environment. 

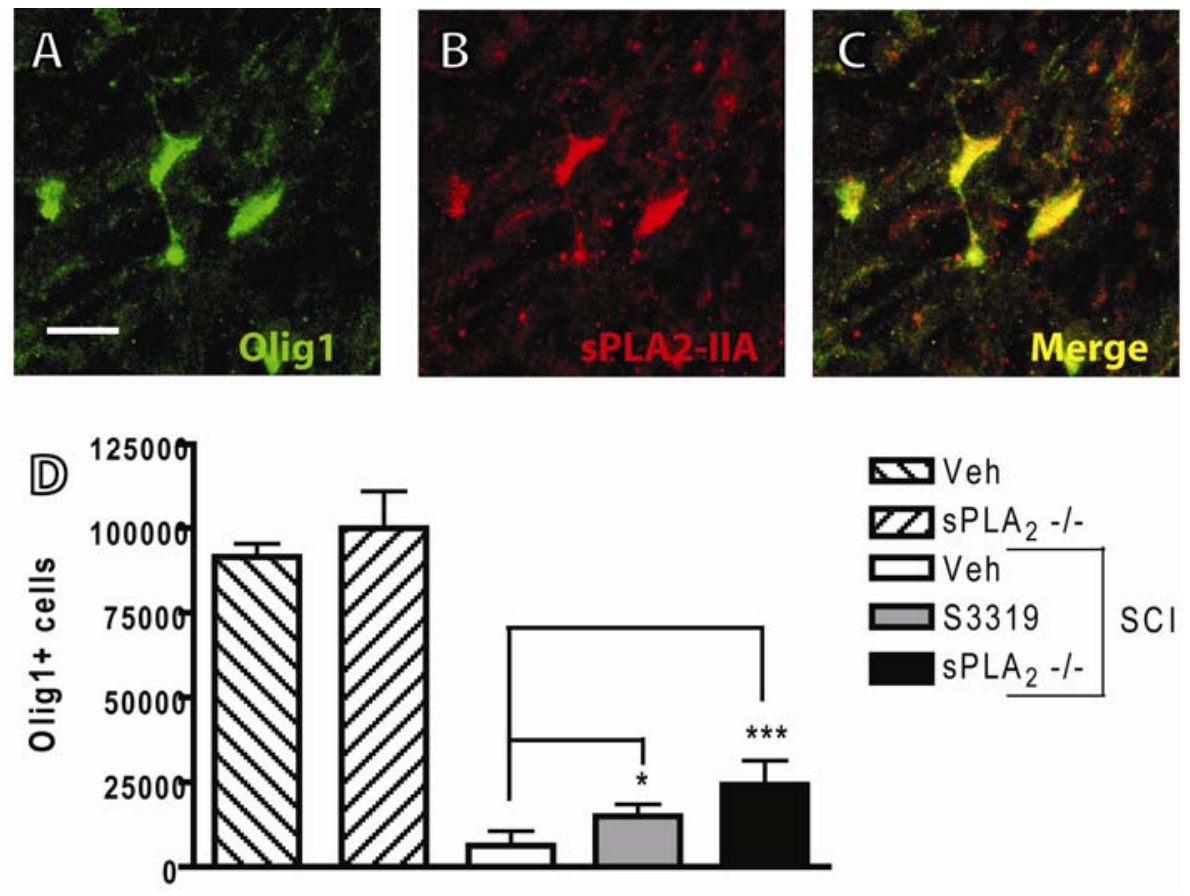

Figure 26. sPLA Inhibition Increases Oligodendrocytes Numbers Following SCI. A-C) Confocal images showing oligodendrocytes (A; Olig1+) co localized with sPLA2IIA (B) in the ventral white matter. D) Mice with decreased sPLA 2 activity either by S3319 inhibition or by a frame shift mutation $\left(\mathrm{sPLA}_{2}-/-\right)$ have significantly more Olig1+ oligodendrocytes within a $4 \mathrm{~mm}$ section of cord centering on the epicenter at 9 weeks post injury. 
Finally, the week 9 BMS scores and the date of voluntary bladder control were collected for each animal and normalized. Then the normalized scores were averaged to produce an average behavior score for each animal at week 9. Then each histological outcome measured in this study was compared to the behavior score to insure that there were no outliers or non-responders, to confirm the precision of the injury device, and to assess the amount of correlation between the outcomes and behavioral improvement. The presence of SMI-31 positive axons at the lesion epicenter $(r(23)=0.87, \mathrm{p}<0.0001)$, the amount of spared white matter $(\mathrm{LFB} ; r(23)=0.95, \mathrm{p}<0.0001)$, the number of Olig $1+$ oligodendrocytes $(r(22)=0.90, \mathrm{p}<0.0001)$ all correlated highly with behavioral outcomes at nine weeks. Accordingly the size of the GFAP negative lesion area showed a similarly strong negative correlation with behavioral function at 9 weeks $(r(23)=-0.90, \mathrm{p}<0.0001)$. Since the inclusion of normal controls in Pearson product moment correlation can artificially inflate significance the analysis was repeated with non-injured animals removed and again each histological outcome highly correlated with behavioral outcomes at nine weeks (SMI-31, $r(16)=0.70, \mathrm{p}<0.01$; LFB, $r(16)=0.87, \mathrm{p}<0.0001$; Olig $1+, r(16)$ $=0.72, \mathrm{p}<0.01$; GFAP, $r(16)=-0.75, \mathrm{p}<0.001)$. While all measures demonstrated a high significance the amount of spared myelin showed the highest level of correlation with behavior function at 9 weeks. Additionally, despite the high severity of the injury, which most likely muted recovery, the high correlations testify to the precision of both the histological quantification and the method of injury. 


\section{Figure 27. Comparison of Histological Outcomes to Behavioral Function}

Each of the histological measures used in this study showed a high level of correlation with the behavioral outcomes at nine weeks. Histological measures are reported as the average normalized percentage. A) Normalized volume of spared white matter in an 8 mm segment of cord. B) shows the normalized volume of GFAP negative lesion within a $4 \mathrm{~mm}$ segment. C) Normalized number of SMI-31+ axons at the lesion epicenter. D) An enlargement of the dotted box in $\mathrm{C}$ that truncates the graph to remove non-injured animals presenting a more accurate representation. E) Number of Olig1+ oligodendrocytes in a $4 \mathrm{~mm}$ segment of cord. Note the relatively tight correlation present in each graph. Note only 6 animals are shown for the S3319 group because the two highest functioning animal were lost after sacrifice due to tissue damage during slicing. 
A

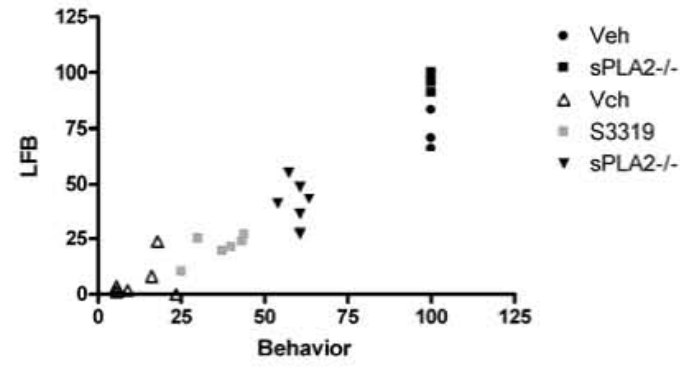

C

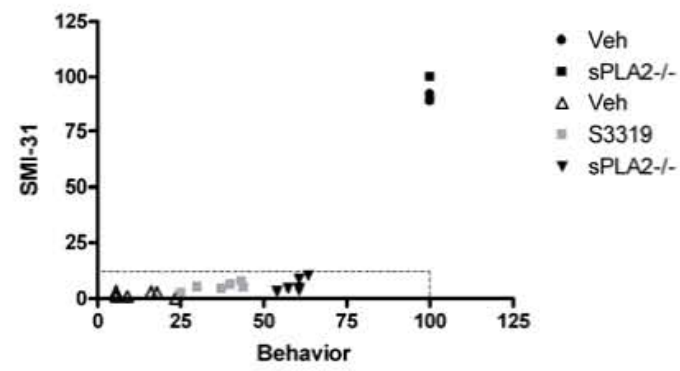

E

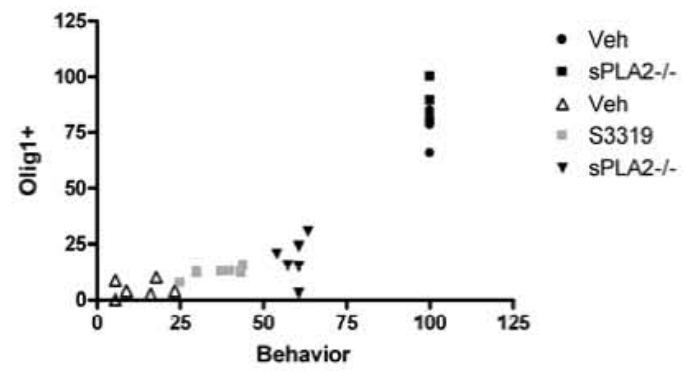

B

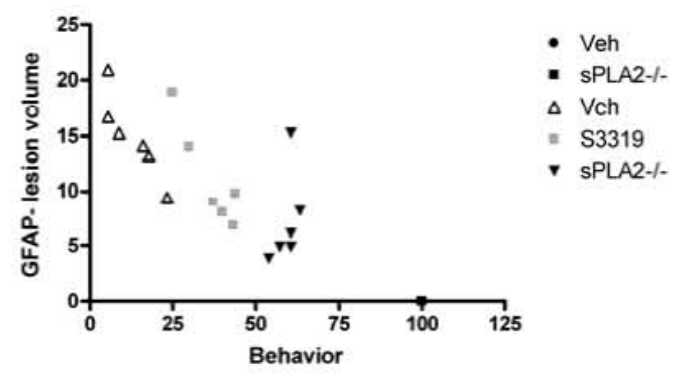

D

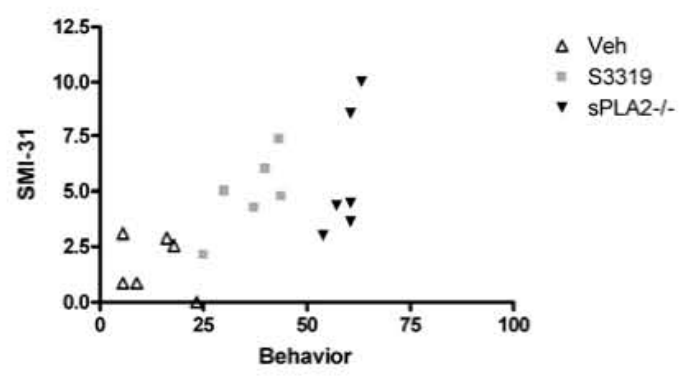




\section{DISCUSSION}

Previously we have shown that sPLA $_{2}$-IIA and IIE are increased following SCI and that direct injection of nanogram doses of $\mathrm{SPLA}_{2}$ results in demyelination and loss of oligodendrocytes within the white matter. However, a gain of function had yet to be demonstrated following the inhibition of $\mathrm{sPLA}_{2}$. In this study we were the first to show that inhibition of $\mathrm{SPLA}_{2}$-IIA prior to severe neurotrauma results in moderate but significant sparing of behavioral function and histology. Inhibition resulted in significantly improved overground locomotion after pharmacological inhibition by the small molecule S3319. Additionally, those mice with muted $\mathrm{SPLA}_{2}$ activity showed on onset of a neurogenic bladder 2-4 weeks before vehicle control mice although it is unclear if this result is due to systemic effect of $\mathrm{SPAA}_{2}$ inhibition.

The most important finding of this study was the significant functional recovery observed after S3319 inhibition. Moreover this recovery correlated highly with tissue sparing. Upon histological examination, significant differences were found in white matter sparing rostral to the lesion, increased sparing of axons at the lesion epicenter, decreased size of lesion volume, and increased number of spared oligodendrocytes within the lesion. The high correlation between behavioral and histological measures served two purposes. First it supports the behavioral observations by external validation and secondly it suggests that the mechanism of functional recovery might be through either direct neuroprotection or tissue repair. Two factors point to the possibility that S3319 might facilitate regeneration. First, the severe spinal cord injury model used in this experiment would lead one to believe that most axons were destroyed by mechanical trauma and therefore these axons would not be candidates for neuroprotection. However, 
S3319 treatment resulted in a doubling of axon profiles within the lesion epicenter. This is exciting since many other authors have found that only modest improvements in axons passing through the lesion can result in significant motor gains. The circuitous nature of these axons and the relative distribution of these fibers throughout the entire spinal cord suggest regenerating fibers. Secondly, the gradual increase of function over 9 weeks and the lack of any significant gains until weeks 7 through 9 suggests a regenerating process rather than a neuroprotection which would show a dramatic increase following recovery from spinal shock and a leveling off of gains after week 3 . However, treatment with S3319 could have protected only a ring of spared axons along the ventral periphery of the spinal cord. More conclusive methods such as axonal tracing following contusion would be required to determine if the observed recovery is from regeneration or neuroprotection. See Chapter V for a more rigorous discussion of regeration.

One limitation of the current study is that we pretreated the animals with S3319. In the present study we utilized pretreatment with S3319 rather than post treatment to seek a "proof of principle" for sPLA $\mathrm{P}_{2}$ inhibition in SCI. Further studies will be conducted to test its efficacy and optimal time window for post-injury treatment. Our work does indicate a possible window of efficacy though. In the initial studies of $\mathrm{sPLA}_{2}$ expression (See study \#1; chapter 2), rats showed peak expression at $4 \mathrm{hr}$ post injury with levels returning to baseline at 1 week. While this allows a short window of efficacy, this is not beyond the realm of current clinical protocols. For example, the standard protocol in most certified stroke centers for patients with ischemic stroke is reperfusion within $3 \mathrm{hr}$ of onset with intravenous recombinant tissue plasminogen activator (TPA; alteplase), 0.9 $\mathrm{mg} / \mathrm{kg}$ over $1 \mathrm{hr}$ which has been shown to reduce death and dependency (odds ratio 0.64 , 
95\%CI 0.5-0.8) (Hankey, 2007b, Hankey, 2007a). In contrast, sPLA 2 -IIA levels, which peak at $4 \mathrm{hrs,} \mathrm{do} \mathrm{not} \mathrm{return} \mathrm{to} \mathrm{baseline} \mathrm{levels} \mathrm{until} \mathrm{one} \mathrm{week} \mathrm{after} \mathrm{injury,} \mathrm{suggesting} \mathrm{an}$ even longer window of opportunity if inhibition of this enzyme is deemed effective. One possible merit of S3319 in a clinical setting is its oral activity, suggesting that the average time to treatment initiation could be shortened even more than TPA drugs which require intravenous administration by a specialist in a controlled setting.

Whether S3319 penetrates into the CNS in sufficient quantities to decrease sPLA 2 activity still remains an important control to perform. However, it is likely that S3319 will be present in sufficient doses. First, S3319 has been shown to be an extremely potent inhibitor of recombinant human $\mathrm{SPLA}_{2}$-IIA with an $\mathrm{IC}_{50}=0.029 \mu \mathrm{m}$ (Hansford et al., 2003). Additionally S3319 has been shown to be orally active and efficacious in the treatment of reperfusion injuries in vivo (Arumugam et al., 2003). Finally, the small molecular weight (487.63) and extreme lipophilic nature of S3319 should facilitate its passage across the blood brain barrier. However, what remains to be conclusively proven is whether the given dose in this study results in decreased $\mathrm{SPLA}_{2}$ activity within the injury site following SCI. This can be achieved by assessing the $\mathrm{SPA}_{2}$ activity present in homogenized spinal tissues by a chromatic assay similar to that which was used in our lab to assess total PLA 2 activity (Liu et al., 2006). The study in question is currently underway.

An alternate explanation for the effects of S3319 in these animals is an inhibition of systemic inflammation rather than as a centrally acting agent. For example, the delay in initiation of neurogenic bladders in the injured animals treated with vehicle could be explained by increased inflammation within the bladders. Bladder inflammation 
following SCI is common secondary to infections due to loss of bladder function. In a study of human SCI patients, bladder biopsies revealed that $91.5 \%$ of patients had inflammation of the bladder wall with $67 \%$ showing chronic inflammation hallmarks (Janzen et al., 2001). This inflammation could then prevent bladder emptying or inhibit the bladder emptying reflex thus resulting in larger voiding volumes when bladders are emptied in comparison to the S3319 treated animals. Muting of systemic inflammation could possible explain the BMS scores as well, in that animals treated with S3319 could have lower inflammation of the extremities and thus more movement following SCI. This explanation is less likely since no evidence has been found that SCI increases joint inflammation of the extremities in either humans or animals. While osteopenia and osteoporosis are common problems in SCI (Jiang et al., 2006, Maimoun et al., 2006) there is no reason to assume that $\mathrm{S} 3319$ would of altered bone densities of calcium handling in the body. A possible method for excluding these possibilities would be to only administer S3319 through an intrathecal pump thus limiting its systemic effects.

The beneficial effects of $\mathrm{SPLA}_{2}-/$ data come with one major caveat. During the initial characterization of the Basso Mouse Scale, it was noted that different strains of mice performed significantly better following a standard contusion. Interestingly, in their original study 3 of the $4 \mathrm{sPLA}_{2}-/$ - mice strains scored significantly better than the only sPLA $_{2}+/+$ strain (Basso et al., 2006). However, the fact that differing strains demonstrated various recoveries following moderate SCI suggests that the differences observed in this study could be due to the sPLA 2 gene or any other genetic difference between the two strains of mice used. While the data presented here does support our hypothesis it does not conclusively demonstrates a role for $\mathrm{sPLA}_{2}$-IIA in spinal cord 
injury. Therefore future studies should use solid genetic techniques to conclusively determine whether sPLA $\mathrm{P}_{2}$-IIA is specifically responsible for the observed effects. Another question that was beyond the scope of this study was the mechanisms behind the functional gains associated with sPLA 2 -IIA inhibition. It is currently unknown whether the gain of function resulted from the prevention of direct cytotoxicity on cells such as oligodendrocytes, a more general suppression of the immune response, or from a facilitation of axonal sprouting through the lesion epicenter. These questions will remain for future studies and must be at least partially addressed in an immunosuppressed mouse model. None the less, the use of a sPLA 2 inhibitor did result in at least partial sparing of the secondary injury following spinal cord injury and merits further investigation. 


\section{CHAPTER V}

\section{DISCUSSION}

Review of Findings

This research began with a simple question posed to me. "What is the brain made of?" Fat is the answer. The CNS is roughly $60 \%$ fat and has a disproportionately high concentration of lipids compared to other tissues of the body and $44 \%$ of those lipids are phospholipids (Morell, 1984). Therefore any destruction of the CNS would require in part the destruction of lipids in general and phospholipids in particular. Many experiments have proven this to be true. Following severe SCI biphasic increases in free fatty acid levels is seen, with levels peaking at 15 min and $24 \mathrm{hr}$ post-trauma before declining over the next 6 days (Demediuk et al., 1989). Even within the first few minutes after SCI, free fatty acids have increased in the grey matter and later increase within the white matter (Demediuk et al., 1985, Faden et al., 1987, Demediuk et al., 1989). These findings suggested that acute $\mathrm{PLA}_{2}$ activity may be present following SCI.

Aside from the obvious effects of phospholipases activity on membrane breakdown, $\mathrm{PLA}_{2}$ presents a tantalizing therapeutic target since its metabolites are also neurotoxins. $\mathrm{PLA}_{2}$ hydrolyze the ester bond at the sn-2 position of membrane phospholipids producing a free fatty acid, such as arachidonic acid (AA), and a lysophospholipid, such as lysolechithin (a.k.a. lysophosphatidyl choline, L-PC). AA has been shown to damage cultured oligodendrocytes and neurons (Wang et al., 2004) and LPC 
can induce demyelination of the CNS and PNS (Blakemore et al., 1977) and act as a macrophage chemoattractant (Lauber et al., 2003). Therefore, our lab investigated the role of phospholipases $\mathrm{A}_{2}$ following SCI. First the total phospholipase activity was shown to increase following SCI and peak at four hours post injury. It was next determined that the well know cytosolic PLA $2\left(\mathrm{CPLA}_{2}\right)$ increased following SCI but cPLA $_{2}$ protein expression did not peak until 7 days post injury, well after total PLA 2 activity crested. This paradox led us to believe that another PLA $\mathrm{A}_{2}$ isozyme was most likely responsible for the majority of phospholipase $A_{2}$ activity seen following injury. Therefore we began to investigate the $\mathrm{PLA}_{2}$ subfamily of enzymes.

We first showed that $\mathrm{sLA}_{2}$ mRNAs and proteins were present in the mammalian nervous system and increased following neurotrauma and cytotoxic injury. While others have demonstrated the presence of selected sPLA 2 mRNAs in the brain (Molloy et al., 1998, Kolko et al., 2005, Kolko et al., 2006) our study was the first demonstration that several sPLA $\mathrm{A}_{2}$ isoforms are present in oligodendrocytes at low levels in the naïve mammalian spinal cord. Additionally, this was the first study to identify increases in ${ }_{\text {sPLA }}$ protein synthesis and expression following neurotrauma. Interestingly, only group II mRNA and protein levels show an increase following SCI. Most importantly, sPLA $2_{2}$ IIA protein peaked at $4 \mathrm{hr}$ post injury in conjunction with the peak of total phospholipase activity suggesting that it might be the phospholipases responsible for a majority of activity increase following neurotrauma. Similarly, the induction of $\mathrm{sPLA} \mathrm{P}_{2}$ synthesis following nervous system injury was confirmed in vitro. We showed that cultured spinal oligodendrocytes increase $\mathrm{sPLA}_{2}$-IIA expression when challenged with proinflammatory molecules present in spinal cord injury such as TNF $\alpha$ and IL-1 $\beta$ or $\mathrm{H}_{2} \mathrm{O}_{2}$. While other 
authors have shown an increase in AA production following $\mathrm{H}_{2} \mathrm{O}_{2}$ injury in other cell lines (Han et al., 2003), this was the first study to directly link free radical injury to sPLA $_{2}$-IIA production. Other authors had already confirmed that sPLA 2 -IIA present in $\mu \mathrm{M}$ levels could trigger apoptosis in cultured neurons (Yagami et al., 2002b) and we showed that exogenously adding sPLA 2 triggered a loss of oligodendrocyte process extensions at low doses and death at $\mu \mathrm{M}$ levels as well. Most importantly, pharmacological inhibition of $\mathrm{sPLA}_{2}$ appears to partially ameliorate oligodendrocyte death following challenge with TNF $\alpha$ and IL- $1 \beta$ or $\mathrm{H}_{2} \mathrm{O}_{2}$ suggesting a critical role for sPLA $_{2}$ in the convergence of injurious signals.

After confirming the upregulation of sPLA $\mathrm{A}_{2}$ following SCI and cell culture, we next determined that increases in $\mathrm{SLA}_{2}$ resulted in a loss of function. To this end we showed that direct injections of nanogram doses of $\mathrm{sPLA}_{2}$ into the naïve spinal cord resulted in extensive demyelination, axonopathy, oligodendrocyte death, and immune cell infiltration. Not surprisingly the tissue damage correlated with a behavioral loss of function in both the pellet reaching behavior and footprint analysis. Interestingly, the observed damage remained predominantly within the white matter when injected at the grey and white matter intersection. This led us to believe that blockade of $\mathrm{sPLA}_{2}$ following SCI could partially ameliorate the spreading white matter degeneration observed with secondary spinal cord degeneration.

Finally, we needed to determine if inhibition of $\mathrm{SLA}_{2}$ in conjunction with SCI resulted in an observable gain of function. While sPLA $\mathrm{P}_{2}$ inhibition had shown neuroprotective effects following a stroke model in rodents (Adibhatla et al., 2006), this was the first study to demonstrate its efficacy following neurotrauma in general and the 
first demonstration of S3319's efficacy in the CNS. In our final set of studies, we showed that pharmacological inhibition of sPLA $_{2}$ decreased inflammation and GFAP negative lesion cavity, as well as increased white matter sparing, oligodendrocyte survival, and axons coursing through the lesion epicenter. Additionally, we showed that inhibition of $\mathrm{SPLA}_{2}$ resulted in a very small but statistically significant gain of function in voluntary over ground locomotion and an increase in bladder voiding volumes $2-4$ weeks earlier than controls.

Another interesting observation is the apparent specificity of sPLA's action in the CNS to post mitotic cells. Previous work by other authors demonstrated SPLA 2 -IIA expression in neurons and its ability to trigger apoptosis in the $\mu \mathrm{M}$ range (Yagami et al., 2002b). In study \#1 (chapter 2) we demonstrated that oligodendrocytes express $\mathrm{sPLA}_{2}$ IIA when injured and that it can induce cytotoxicity at levels identical to those seen in other studies utilizing neurons. Interestingly, astrocytes and Schwann cells show no increase in LDH or decrease in MTT production following injury with $2 \mu \mathrm{M}$ of sPLA $_{2}-$ IIA. This indicates that inhibition of sPLA $\mathrm{A}_{2}$ following injury could be specific to neurons and oligodendrocytes and could explain why lesioning of the spinal cord with injections of $\mathrm{SPLA}_{2}$ results in a death of oligodendrocytes but a gliosis and remyelination with Schwann cells (see study \#2; chapter 3). Within study \#3 (chapter 4), this idea is supported by the increased number of oligodendrocytes and axon profiles seen after S3319 treatment. However exact quantification of neurons within the lesion needs to be performed.

\section{Limitations of Current Findings}


The first study simply designed and executed; however some comments must be made about the specificity of our sPLA 2 -IIA inhibitor, S3319. S3319 was specifically developed as a highly potent inhibitor of sPLA 2 -IIA. This was done by designing a theoretical back bone that would intercalate into the group IIA enzymatic site. Then the side chain structures were varied to empirically determine the compound with the highest affinity and lowest $\mathrm{Kd}$. In the orinal publication detailing this molecule it was stated that a $2.2 \AA$ crystal structure of the resulting structure shows ...

“... an inhibitor that is bound in the active site of the enzyme, chelated to a $\mathrm{Ca}^{2+}$ ion through coarboxylate and amide oxygen atoms, $\mathrm{H}$-bonded through an amide $\mathrm{NH}$ group to His48, with multiple hydrophobic contacts, and a T-shaped aromatic - His6 interaction.” (Hansford et al., 2003)

While the highly specified binding site and 3-D structure analysis suggest a selective inhibition of IIA over other sPLA ${ }_{2}$ S, S3319's activity against other isoforms has not been directly assessed. In general the use of pharmacological agents in studies introduces the possibility that said agent can have unintended activities on other molecules, often times wholly unrelated to the target of inhibition. Consequently these unintended interactions are the result of the observed effects. However, the high potency of S3319, low concentrations used, and its unique development suggest that the observed effects were from sPLA 2 -IIA inhibition. However, future studies should confirm the above observations either through knock down or knock out studies such as was shown by Han et al., (2003). 
In the second study, $\mathrm{sPLA}_{2}$-III was used to assess the effects of $\mathrm{SPLA}_{2}$ activity on the naïve central nervous system. sPLA $_{2}$-III extracted from bee venom and was selected because its enzymatic activity is well characterised and quantified and therefore could be used to reproduce the total phospholipase activity that was calculated in earlier studies (Liu et al., 2006). However, in the first study one can note that sPLA 2 -III was not found in the rat CNS and to date a mammalian group III has only been confirmed in humans (Valentin et al., 2000a). Interestingly both human group III (Masuda et al., 2008) and group $\mathrm{X} \mathrm{sPLA}_{2}$ (Ikeno et al., 2005) have been shown to increase neurite outgrowth in cultured PC12 cells at very low doses. These finds further complicate the generalizability of $\mathrm{SPLA}_{2}$ inhibition following SCI, especially if $\mathrm{S} 3319$ or any other $\mathrm{SPLA}_{2}$ inhibitor is found to have non-specific inhibition. However, the fact that histological changes were seen in the third study following S3319 treatment in SCI suggests that either the drug is specific for $\mathrm{sPLA}_{2}$-IIA or that the detrimental effects of IIA outweigh the neurogenic properties of groups III and X. None the less it will be crucial to evaluate the specificity of an inhibitor on $\mathrm{sPLA}_{2}$ isoforms before studies are performed in higher animals. The cross inhibition of various inhibitors could help to explain the mixed success of previous sPLA $_{2}$-IIA inhibitors in clinical trials (Reid, 2005).

In the third study there are several matters of extreme concern that limit the inclusion of any of the $129 \mathrm{svj}\left(\mathrm{sPLA}_{2} /-\right.$ ) mice in future publications resulting from this work. First any comparison of $\mathrm{SPLA}_{2}-/-$ mice to those possessing the $\mathrm{PLA}_{2}$-IIA gene must be called into question because no proper control was included for the $\mathrm{SLA}_{2}-/-$ mice. Therefore any differences observed between the SCI groups could be attributable to either sPLA 2 -IIA or any of the thousands of other genetic variations between these 
species. Secondly, due to the poor design of this experiment, we elected not confirm the

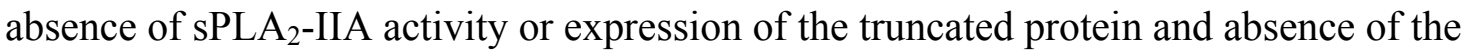
full length protein in these animals.

In a broader sense the lack of any developmental defects in $129 \mathrm{svj}$ mice suggests that sPLA 2 -IIA may not play a crucial role in the CNS. However, I would argue that this is not true. If, as we suggest here, that $\mathrm{SLA}_{2}$-IIA is primarily an initiator and/or propagator of inflammation, than one would expect to see few defects in a normally reared animal without pathology. To this end, I feel that any constitutive $\mathrm{KO}$ animal is a poor model for SCI investigation. It makes little since to deprive an animal of a gene for its entire development when one is interest in an acute event such as SCI or stroke.

Following injury if a difference is detected it is unclear whether this observation is due to loss of function during the time of injury (and therefore a suitable therapeutic target) or rather a result of altered development (and of less utility). In my opinion it is of greater value to utilize one of the inducible $\mathrm{KO}$ systems such as utilizing doxycycline in a "TetOff" system to inhibit sPLA2-IIA expression just prior to SCI (Kistner et al., 1996). Unfortunately this strain has yet to be developed.

In any paper showing functional improvements the likelihood of regeneration should be rigorously addressed. An article by Steward, Zheng, and Tessier-Lavigne (2003) laid out criteria for distinguishing regenerated axons from spared axons in the central nervous system. They are as follows;

(I) The axon extends from the CNS into a non-CNS environment, specifically, the tissue environment of the scar that develops at the injury site. 
(II) The axon extends from the host CNS into a nonhost graft or transplant.

(III) The axon originates at or near a site of amputation.

(IV) The axon takes an unusual course through the tissue environment of the CNS.

(V) The axon extends no further than could be accounted for by plausible regeneration rates.

(VI) The axon is tipped with a growth cone.

(VII) The axon has a morphology that is not characteristic of normal axons of its type (for example exhibiting unusual branching patterns). (Steward et al., 2003)

Since axons were seen evenly spaced throughout the lesion epicenter, GFAPcore, and axons are present in what should be both grey and white mater criteria I and IV are met suggesting regeneration. Secondly, since histological samples were not made until 9 weeks post injury and since behavioral recovery was not seen until weeks 7 - 9, it can be assumed that if regeneration occurred and it is playing a role in any observed functional increases, it did so at plausible growth rate (criteria V). However, since the injury model was a contusion and not hemisection and no graft tissue was placed in the lesion site, criteria II and III are irrelevant. Finally, the presence of growth cones and the branching patterns were not investigated due to the age of the lesion and that no tracing was performed in this study leaving criteria VI and VII unknown. Taken in total increased regeneration could have happened in the S3319 treated animals, but there is simply insufficient evidence to suggest that it occurred based on the data presented here. 
Application of Findings to Future sPLA 2 Research:

It must be noted that $\mathrm{SPLA}_{2}$ does not function as a phospholipase in isolation. Rather than focusing on sPLA2 in CNS injury, current literature has focused on role of cPLA 2 in ischemia/stroke (Bonventre et al., 1997, Saluja et al., 1997, Arai et al., 2001, Tabuchi et al., 2003), multiple sclerosis, and the mouse correlate experimental autoimmune encephalomyelitis (Kalyvas and David, 2004, Marusic et al., 2005). However, there appears to be a reciprocal accentuation of activity among the PLA $\mathrm{A}_{2} \mathrm{~S}$ (Murakami et al., 1998, Han et al., 2003). This suggests that inhibition of sPLA 2 , cPLA and possibly even iPLA 2 in unison could result in a synergistic increase in neurological sparing which merits further study. Additionally, variations in the biological functions and species specificities of various $\mathrm{SPLA}_{2}$ isoforms complicate the issue further (Cupillard et al., 1999, Kudo and Murakami, 2002).

For example, the rabbit $\mathrm{SLA}_{2}-\mathrm{R}$ is very promiscuous, binding to almost all sPLA $_{2}$ tested to date. The mouse $\mathrm{SPLA}_{2}-\mathrm{R}$ on the other hand binds only IB, IIA, and X, while the rat sPLA 2 -R only binds sPLA 2 -IB (Cupillard et al., 1999). Likewise sPLA 2 -IIA does not seem to bind to the sPLA $2-\mathrm{R}$ in humans (Cupillard et al., 1999). What seems to be important is that within each species at least one isoform of $\mathrm{SPA}_{2}$ plays an important role in inflammation. In mice that lack sPLA $\mathrm{PLA}_{2}$-IIA activity, $\mathrm{SPLA}_{2}-\mathrm{V}$ takes it place in the propagation of the immune response to infection (Kudo and Murakami, 2002). However, any further work in SCI would need cautious experimentation up the phylogenetic tree before experiments in humans could be initiated. 
Unpublished work from our lab has confirmed that $\mathrm{SPLA}_{2}$-IIA is at least present in the human CNS after SCI. However, any findings found in sub-human species will need to be interpreted conservatively when applied to preclinical research.

Future Directions

$\mathrm{sPLA}_{2}$, particularly group IIA, shows promise as a therapeutic target. It has been strongly associated with many types of inflammation and disease states in various species(Kudo and Murakami, 2002). These studies indicate that sPLA 2 -IIA might play a role in SCI and that its inhibition could result in some histological and functional sparing. However work still remains in unlocking the mechanism of action and pushing SPLA2 inhibition from the bench to the bedside.

First sPLA 2 -IIA action needs to be confirmed by genetic manipulation with a controllable down regulation. This will involve not only measuring the expression of sPLA 2 -IIA protein but the enzymatic function of $\mathrm{sPLA}_{2}$ following knock down. The latter experiment will aid in determining group IIA's role in total sPLA ${ }_{2}$ and total $\mathrm{PLA}_{2}$ activity. Secondly, this will remove any ambiguity that results from use of a pharmacological agent and its possible actions on other $\mathrm{PLA}_{2} \mathrm{~S}$ or unrelated molecules.

Secondly, the pharmacological effects of S3319 in the CNS will need to be investigated further. While protein expression suggests at least a $4 \mathrm{hr}$ window, S3319 will need to be administered at different time points after SCI to determine if a therapeutic window does exist and how long it takes S3319 to buildup to adequate levels in brain tissue. Additionally, while the serum half-life of $\mathrm{sPLA}_{2}$ is rather short (12 24hr); investigating the half life of S3319 enzymatic inhibition in the CNS could easily 
yield a lower optimal dose. It is easy to imagine that the lipophilic nature of S3319 could result in build up in fatty tissues.

Third, the mechanism of S3319 inhibition in SCI should be investigated further. In general three mechanisms of action could be envisioned with the data available; observable gains following sPLA2-IIA inhibition could result from 1) decreased inflammation, 2) increased axon regeneration across the lesion site, or 3) inhibition of direct cytotoxicity to neurons and/or oligodendroglia.

While the current study showed increased axons at the lesion epicenter, it is unknown whether these axons are regenerative and whether these axons contribute to functional recovery. The most crucial study will be to compare the retrograde labeled axons in a more controlled lesion. For example counting the number of anterograde labeled axons coursing through a lesion and the number of retrograde labeled neurons in central nuclei following selective tract lesioning in either treated or vehicle animals. This study could additionally be augmented with a relesioning group to demonstrate a return to baseline of any observable gains.

Given sPLA 2 -IIA's role in inflammation and S3319's previously observed antiinflammatory effects its role in inflammation should be further investigated. First, simple quantification of OX-42 positive macrophages and microglia in the current tissue sections should be assessed if adequate samples remain. Additionally, replication of this study in immunologically suppressed animals would help elucidate what role the antiinflammatory effects of S3319 play in the observed histological changes. Similar studies have already been performed in SCI using either administration of immunosuppressant (Rabchevsky et al., 2001, Nottingham et al., 2002) or depletion of peripheral immune 
cells (Popovich et al., 1999). These experiments would better determine the contribution of $\mathrm{SPLA}_{2}$-IIA in the mobilization of the immune response. Of course the possibility exists that all of the aforementioned mechanisms or some combination therein, contribute to the observed behaviors in which case testing of each mechanism will show partial involvement.

In the third study it was noticed post hoc that the animals given S3319 showed subjectively smaller bladder volumes on twice daily voiding. While the effect was dramatic, it needs to be repeated and more rigorously studied. Evaluation of lower urinary tract function by urodynamic cystometry would allow for measures of contraction amplitudes and duration, intercontraction interval, voiding pressure, while use of a Ballman cage would allow rigorous quantification of voided volumes and residual bladder volumes and would more readily confirm the above observation (Leung et al., 2007). Also histological sampling of the bladder wall could show that S3319, systemically administered medication, could have simply reduced cystitis and thus facilitated the neurogenic bladders.

Finally, the intracellular mechanisms utilized by oxidative stressors and cytokines to upregulate $\mathrm{sPLA}_{2}$ after SCI remain obscure. In several cell types, $\mathrm{SPLA}_{2}$-IIA expression is dependent on prior activation of $\mathrm{cPLA}_{2}-\alpha$, where certain products generated by the cPLA $2 \alpha-12 / 15$-lipoxygenase pathway may play a critical role (Kuwata et al., 1998, Couturier et al., 1999, Kuwata et al., 2000). It has been demonstrated in murine mesangial cells that $\mathrm{H}_{2} \mathrm{O}_{2}$ injury upregulates MEK-1 and ERK 1/2 that in turn stimulates cPLA $2-\alpha$ production. $\mathrm{cPLA}_{2}-\alpha$ was subsequently shown to increase $\mathrm{PLA}_{2}$-IIA expression via the 12/15 lipoxygenase pathway and form a positive feedback loop 


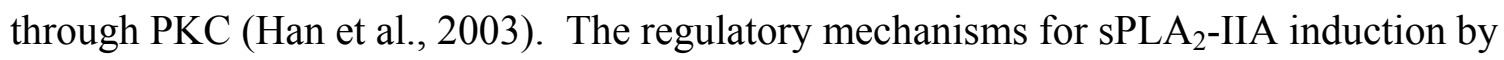
cytokines differ according to cell type. For example, the activation of NF-kB, C/EBP $\beta$ and $\mathrm{C} / \mathrm{EBP} \delta$, and STAT3 are necessary for $\mathrm{SPLA}_{2}$-IIA induction in rat mesangial cells, rabbit chondrocytes, and human arterial smooth muscle cells respectively (Kennedy et al., 1995, Massaad et al., 2000). Therefore further studies will need to assess the exact mechanism of $\mathrm{sPLA}_{2}$ mediated CNS injury to determine if it is a common convergence mechanism.

\section{Conclusions}

James W. Fawcett, Ph.D. closed his recent seminar at the University of Louisville with a picture of the profoundly complex control panel of the supersonic Concorde Jet. He then explained that current SCI research is equivalent to flipping one switch while the Concorde is flying at top speed. The switch could lower the landing gear which would inadvertently slow the airplane. However, the purpose of the landing gear is not to slow the plane but rather allowing it to land safely. Within this work we hypothesized that the purpose of sPLA2-IIA was to increase inflammation and degrade neural tissue following SCI, which we have shown that it can do. However, whether this is the end all purpose of this molecule remains for further study but what is assured is that the results presented here show a histological and possible behavioral change that merits further study and consideration.

Quod erat demonstrandum

Soli Deo gloria 


\section{REFERENCES}

Abe K, Yuki S, Kogure K (1988) Strong attenuation of ischemic and postischemic brain edema in rats by a novel free radical scavenger. Stroke 19:480-485.

Adibhatla RM, Hatcher JF (2006) Phospholipase A2, reactive oxygen species, and lipid peroxidation in cerebral ischemia. Free Radic Biol Med 40:376-387.

Adibhatla RM, Hatcher JF (2007) Secretory phospholipase A2 IIA is up-regulated by TNF-alpha and IL-1alpha/beta after transient focal cerebral ischemia in rat. Brain Res 1134:199-205.

Adibhatla RM, Hatcher JF, Larsen EC, Chen X, Sun D, Tsao FH (2006) CDP-choline significantly restores phosphatidylcholine levels by differentially affecting phospholipase A2 and CTP: phosphocholine cytidylyltransferase after stroke. J Biol Chem 281:6718-6725.

Ancian P, Lambeau G, Mattei MG, Lazdunski M (1995) The human 180-kDa receptor for secretory phospholipases A2. Molecular cloning, identification of a secreted soluble form, expression, and chromosomal localization. J Biol Chem 270:89638970.

Andersen JM, Myhre O, Fonnum F (2003) Discussion of the role of the extracellular signal-regulated kinase-phospholipase A2 pathway in production of reactive oxygen species in Alzheimer's disease. Neurochem Res 28:319-326.

Andersen S, Sjursen W, Laegreid A, Volden G, Johansen B (1994) Elevated expression of human nonpancreatic phospholipase A2 in psoriatic tissue. Inflammation 18:112.

Anderson KD (2004) Targeting recovery: priorities of the spinal cord-injured population. J Neurotrauma 21:1371-1383.

Arai K, Ikegaya Y, Nakatani Y, Kudo I, Nishiyama N, Matsuki N (2001) Phospholipase A2 mediates ischemic injury in the hippocampus: a regional difference of neuronal vulnerability. Eur J Neurosci 13:2319-2323.

Arita H, Hanasaki K, Nakano T, Oka S, Teraoka H, Matsumoto K (1991) Novel proliferative effect of phospholipase A2 in Swiss 3T3 cells via specific binding site. J Biol Chem 266:19139-19141.

Arnett HA, Fancy SP, Alberta JA, Zhao C, Plant SR, Kaing S, Raine CS, Rowitch DH, Franklin RJ, Stiles CD (2004) bHLH transcription factor Olig1 is required to repair demyelinated lesions in the CNS. Science 306:2111-2115.

Arumugam TV, Arnold N, Proctor LM, Newman M, Reid RC, Hansford KA, Fairlie DP, Shiels IA, Taylor SM (2003) Comparative protection against rat intestinal reperfusion injury by a new inhibitor of sPLA2, COX-1 and COX-2 selective inhibitors, and an LTC4 receptor antagonist. Br J Pharmacol 140:71-80. 
Azbill RD, Mu X, Bruce-Keller AJ, Mattson MP, Springer JE (1997) Impaired mitochondrial function, oxidative stress and altered antioxidant enzyme activities following traumatic spinal cord injury. Brain Res 765:283-290.

Balboa MA, Shirai Y, Gaietta G, Ellisman MH, Balsinde J, Dennis EA (2003) Localization of group $\mathrm{V}$ phospholipase $\mathrm{A} 2$ in caveolin-enriched granules in activated P388D1 macrophage-like cells. J Biol Chem 278:48059-48065.

Balsinde J, Perez R, Balboa MA (2006) Calcium-independent phospholipase A2 and apoptosis. Biochim Biophys Acta 1761:1344-1350.

Bao F, Liu D (2004) Hydroxyl radicals generated in the rat spinal cord at the level produced by impact injury induce cell death by necrosis and apoptosis: protection by a metalloporphyrin. Neuroscience 126:285-295.

Baran H, Heldt R, Hertting G (1987) Increased prostaglandin formation in rat brain following systemic application of kainic acid. Brain Res 404:107-112.

Basso DM, Fisher LC, Anderson AJ, Jakeman LB, McTigue DM, Popovich PG (2006) Basso Mouse Scale for locomotion detects differences in recovery after spinal cord injury in five common mouse strains. J Neurotrauma 23:635-659.

Bate C, Rumbold L, Williams A (2007) Cholesterol synthesis inhibitors protect against platelet-activating factor-induced neuronal damage. J Neuroinflammation 4:5.

Benevento BT, Sipski ML (2002) Neurogenic bladder, neurogenic bowel, and sexual dysfunction in people with spinal cord injury. Phys Ther 82:601-612.

Bezzine S, Koduri RS, Valentin E, Murakami M, Kudo I, Ghomashchi F, Sadilek M, Lambeau G, Gelb MH (2000) Exogenously added human group X secreted phospholipase $\mathrm{A}_{2}$ but not the group IB, IIA, and V enzymes efficiently release arachidonic acid from adherent mammalian cells. J Biol Chem 275:3179-3191.

Blakemore WF (1978) Observations on remyelination in the rabbit spinal cord following demyelination induced by lysolecithin. Neuropathol Appl Neurobiol 4:47-59.

Blakemore WF (1982) Ethidium bromide induced demyelination in the spinal cord of the cat. Neuropathol Appl Neurobiol 8:365-375.

Blakemore WF, Eames RA, Smith KJ, McDonald WI (1977) Remyelination in the spinal cord of the cat following intraspinal injections of lysolecithin. J Neurol Sci 33:3143.

Blight AR (1985) Delayed demyelination and macrophage invasion: a candidate for secondary cell damage in spinal cord injury. Cent Nerv Syst Trauma 2:299-315.

Bonventre JV (1996) Roles of phospholipases A2 in brain cell and tissue injury associated with ischemia and excitotoxicity. J Lipid Mediat Cell Signal 14:15-23.

Bonventre JV, Huang Z, Taheri MR, O'Leary E, Li E, Moskowitz MA, Sapirstein A (1997) Reduced fertility and postischaemic brain injury in mice deficient in cytosolic phospholipase A2. Nature 390:622-625.

Bresnahan JC (1978) An electron-microscopic analysis of axonal alterations following blunt contusion of the spinal cord of the rhesus monkey (Macaca mulatta). J Neurol Sci 37:59-82.

Buki A, Okonkwo DO, Wang KK, Povlishock JT (2000) Cytochrome c release and caspase activation in traumatic axonal injury. J Neurosci 20:2825-2834.

Bullock R, Fujisawa H (1992) The role of glutamate antagonists for the treatment of CNS injury. J Neurotrauma 9 Suppl 2:S443-462. 
Bunge RP, (1994) Clinical implications of recent advances in neurotrauma research. Oxford University Press, New York.

Buntinx M, Moreels M, Vandenabeele F, Lambrichts I, Raus J, Steels P, Stinissen P, Ameloot M (2004) Cytokine-induced cell death in human oligodendroglial cell lines: I. Synergistic effects of IFN-g and TNF-a on apoptosis. J Neurosci Res 76:834-845.

Butterfield DA, Griffin S, Munch G, Pasinetti GM (2002) Amyloid beta-peptide and amyloid pathology are central to the oxidative stress and inflammatory cascades under which Alzheimer's disease brain exists. J Alzheimers Dis 4:193-201.

Cane A, Breton M, Koumanov K, Bereziat G, Colard O (1998) Oxidant-induced arachidonic acid release and impairment of fatty acid acylation in vascular smooth muscle cells. Am J Physiol 274:C1040-1046.

Cao Q, Zhang YP, Iannotti C, DeVries WH, Xu XM, Shields CB, Whittemore SR (2005) Functional and electrophysiological changes after graded traumatic spinal cord injury in adult rat. Exp Neurol 191 Suppl 1:S3-S16.

Cao QL, Howard RM, Dennison JB, Whittemore SR (2002) Differentiation of engrafted neuronal-restricted precursor cells is inhibited in the traumatically injured spinal cord. Exp Neurol 177:349-359.

Chabot C, Gagne J, Giguere C, Bernard J, Baudry M, Massicotte G (1998) Bidirectional modulation of AMPA receptor properties by exogenous phospholipase A2 in the hippocampus. Hippocampus 8:299-309.

Chau CH, Shum DK, Li H, Pei J, Lui YY, Wirthlin L, Chan YS, Xu XM (2004) Chondroitinase ABC enhances axonal regrowth through Schwann cell-seeded guidance channels after spinal cord injury. Faseb J 18:194-196.

Chen J, Engle SJ, Seilhamer JJ, Tischfield JA (1994) Cloning and characterization of novel rat and mouse low molecular weight $\mathrm{Ca}(2+)$-dependent phospholipase A2s containing 16 cysteines. J Biol Chem 269:23018-23024.

Church WB, Inglis AS, Tseng A, Duell R, Lei PW, Bryant KJ, Scott KF (2001) A novel approach to the design of inhibitors of human secreted phospholipase A2 based on native peptide inhibition. J Biol Chem 276:33156-33164.

Clapp LE, Klette KL, DeCoster MA, Bernton E, Petras JM, Dave JR, Laskosky MS, Smallridge RC, Tortella FC (1995) Phospholipase A2 -induced neurotoxicity in vitro and in vivo in rats. Brain Reseach 693:101-111.

Colangelo V, Schurr J, Ball MJ, Pelaez RP, Bazan NG, Lukiw WJ (2002) Gene expression profiling of 12633 genes in Alzheimer hippocampal CA1: transcription and neurotrophic factor down-regulation and up-regulation of apoptotic and pro-inflammatory signaling. J Neurosci Res 70:462-473.

Cormier RT, Hong KH, Halberg RB, Hawkins TL, Richardson P, Mulherkar R, Dove WF, Lander ES (1997) Secretory phospholipase Pla2g2a confers resistance to intestinal tumorigenesis. Nat Genet 17:88-91.

Couturier C, Brouillet A, Couriaud C, Koumanov K, Bereziat G, Andreani M (1999) Interleukin 1 beta induces type II-secreted phospholipase A(2) gene in vascular smooth muscle cells by a nuclear factor kappaB and peroxisome proliferatoractivated receptor-mediated process. J Biol Chem 274:23085-23093. 
Crowe MJ, Bresnahan JC, Shuman SL, Masters JN, Beattie MS (1997) Apoptosis and delayed degeneration after spinal cord injury in rats and monkeys.[erratum appears in Nat Med 1997 Feb;3(2):240]. Nature Medicine 3:73-76.

Crowl RM, Stoller TJ, Conroy RR, Stoner CR (1991) Induction of phospholipase A2 gene expression in human hepatoma cells by mediators of the acute phase response. J Biol Chem 266:2647-2651.

Cupillard L, Koumanov K, Mattâei MG, Lazdunski M, Lambeau G (1997) Cloning, chromosomal mapping, and expression of a novel human secretory phospholipase A2. The Journal of biological chemistry 272:15745-15752.

Cupillard L, Mulherkar R, Gomez N, Kadam S, Valentin E, Lazdunski M, Lambeau G (1999) Both group IB and group IIA secreted phospholipases A2 are natural ligands of the mouse 180-kDa M-type receptor. J Biol Chem 274:7043-7051.

de Almeida LM, Pineiro CC, Leite MC, Brolese G, Leal RB, Gottfried C, Goncalves CA (2007) Protective Effects of Resveratrol on Hydrogen Peroxide Induced Toxicity in Primary Cortical Astrocyte Cultures. Neurochem Res.

de Medinaceli L, Freed WJ, Wyatt RJ (1982) An index of the functional condition of rat sciatic nerve based on measurements made from walking tracks. Experimental Neurology 77:634-643.

DeCoster MA (2003) Group III secreted phospholipase A2 causes apoptosis in rat primary cortical neuronal cultures. Brain Res 988:20-28.

Demediuk P, Daly MP, Faden AI (1989) Changes in free fatty acids, phospholipids, and cholesterol following impact injury to the rat spinal cord. J Neurosci Res 23:95106.

Demediuk P, Saunders RD, Clendenon NR, Means ED, Anderson DK, Horrocks LA (1985) Changes in lipid metabolism in traumatized spinal cord. Prog Brain Res 63:211-226.

Demjen D, Klussmann S, Kleber S, Zuliani C, Stieltjes B, Metzger C, Hirt UA, Walczak H, Falk W, Essig M, Edler L, Krammer PH, Martin-Villalba A (2004) Neutralization of CD95 ligand promotes regeneration and functional recovery after spinal cord injury. Nat Med 10:389-395.

Dennis EA (1994) Diversity of group types, regulation and function of phospholipase A2. Journal of Biological Chemistry 269:13057-13060.

Dhillon HS, Donaldson D, Dempsey RJ, Prasad MR (1994) Regional levels of free fatty acids and Evans blue extravasation after experimental brain injury. J Neurotrauma 11:405-415.

Dorandeu F, Pernot-Marino I, Veyret J, Perrichon C, Lallement G (1998) Secreted phospholipase A2-induced neurotoxicity and epileptic seizures after intracerebral administration: an unexplained heterogeneity as emphasized with paradoxin and crotoxin. J Neurosci Res 54:848-862.

Dumuis A, Sebben M, Haynes L, Pin JP, Bockaert J (1988) NMDA receptors activate the arachidonic acid cascade system in striatal neurons. Nature 336:68-70.

Dutta J, Das AK, Biswas A (1979) Enzymatic reactions on thin-layer chromatographic plates. II. Phospholipase $\mathrm{A}_{2}$ hydrolysis of phosphatidylcholine and separation of the products on a single plate. Journal of chromatography 173:379-387. 
Estevez AY, Phillis JW (1997) The phospholipase A2 inhibitor, quinacrine, reduces infarct size in rats after transient middle cerebral artery occlusion. Brain Reseach 752:203-208.

Everse J, Coates PW (2005) Role of peroxidases in Parkinson disease: a hypothesis. Free Radic Biol Med 38:1296-1310.

Fabia R, Ar'Rajab A, Willen R, Andersson R, Bengmark S (1993) Effect of putative phospholipase A2 inhibitors on acetic acid-induced acute colitis in the rat. Br J Surg 80:1199-1204.

Faden AI, Chan PH, Longar S (1987) Alterations in lipid metabolism, $\mathrm{Na}+, \mathrm{K}+-\mathrm{ATPase}$ activity, and tissue water content of spinal cord following experimental traumatic injury. J Neurochem 48:1809-1816.

Faden AI, Halt P (1992) Platelet-activating factor reduces spinal cord blood flow and causes behavioral deficits after intrathecal administration in rats through a specific receptor mechanism. J Pharmacol Exp Ther 261:1064-1070.

Farooque M, Hillered L, Holtz A, Olsson Y (1996) Effects of methylprednisolone on extracellular lactic acidosis and amino acids after severe compression injury of rat spinal cord. J Neurochem 66:1125-1130.

Farooqui AA, Litsky ML, Farooqui T, Horrocks LA (1999a) Inhibitors of intracellular phospholipase A2 activity: their neurochemical effects and therapeutical importance for neurological disorders. Brain Res Bull 49:139-153.

Farooqui AA, Litsky ML, Farooqui T, Horrocks LA (1999b) Inhibitors of intracellular phospholipase A2 activity: Their neurochemical effects and therapeutical importance for neurological disorders. Brain Reseach Bullitin 49:139-153.

Farooqui AA, Ong WY, Horrocks LA (2004) Biochemical aspects of neurodegeneration in human brain: involvement of neural membrane phospholipids and phospholipases A2. Neurochem Res 29:1961-1977.

Farooqui AA, Ong WY, Horrocks LA (2006) Inhibitors of brain phospholipase A2 activity: their neuropharmacological effects and therapeutic importance for the treatment of neurologic disorders. Pharmacol Rev 58:591-620.

Farooqui AA, Rapoport SI, Horrocks LA (1997a) Membrane phospholipid alterations in Alzheimer's disease: deficiency of ethanolamine plasmalogens. Neurochem Res 22:523-527.

Farooqui AA, Yang HC, Rosenberger TA, Horrocks LA (1997b) Phospholipase A2 and its role in brain tissue. J Neurochem 69:889-901.

Farooqui AA, Yi Ong W, Lu XR, Halliwell B, Horrocks LA (2001) Neurochemical consequences of kainate-induced toxicity in brain: involvement of arachidonic acid release and prevention of toxicity by phospholipase A(2) inhibitors. Brain Res Brain Res Rev 38:61-78.

Fatokun AA, Stone TW, Smith RA (2007) Cell death in rat cerebellar granule neurons induced by hydrogen peroxide in vitro: mechanisms and protection by adenosine receptor ligands. Brain Res 1132:193-202.

Fleming JC, Norenberg MD, Ramsay DA, Dekaban GA, Marcillo AE, Saenz AD, Pasquale-Styles M, Dietrich WD, Weaver LC (2006) The cellular inflammatory response in human spinal cords after injury. Brain 129:3249-3269. 
Fonteh AN, Atsumi G, LaPorte T, Chilton FH (2000) Secretory phospholipase A2 receptor-mediated activation of cytosolic phospholipase A2 in murine bone marrow-derived mast cells. Journal of Immunology 165:2773-2782.

Gelb MH, Valentin E, Ghomashchi F, Lazdunski M, Lambeau G (2000) Cloning and recombinant expression of a structurally novel human secreted phospholipase A2. J Biol Chem 275:39823-39826.

Gennarelli TA (1993) Mechanisms of brain injury. J Emerg Med 11 Suppl 1:5-11.

Giri S, Khan M, Rattan R, Singh I, Singh AK (2006) Krabbe disease: psychosinemediated activation of phospholipase A2 in oligodendrocyte cell death. Journal of lipid research 47:1478-1492.

Green JA, Smith GM, Buchta R, Lee R, Ho KY, Rajkovic IA, Scott KF (1991) Circulating phospholipase A2 activity associated with sepsis and septic shock is indistinguishable from that associated with rheumatoid arthritis. Inflammation 15:355-367.

Gronroos JM, Kuttila K, Perttila J, Nevalainen TJ (1995) Serum phospholipases A2 after aortobifemoral reconstruction. Eur J Clin Chem Clin Biochem 33:271-274.

Guest JD, Hiester ED, Bunge RP (2005) Demyelination and Schwann cell responses adjacent to injury epicenter cavities following chronic human spinal cord injury. Exp Neurol 192:384-393.

Hains BC, Yucra JA, Hulsebosch CE (2001) Reduction of pathological and behavioral deficits following spinal cord contusion injury with the selective cyclooxygenase2 inhibitor NS-398. J Neurotrauma 18:409-423.

Halat G, Lukacova N, Chavko M, Marsala J (1987) Effects of incomplete ischemia and subsequent recirculation on free palmitate, stearate, oleate and arachidonate levels in lumbar and cervical spinal cord of rabbit. Gen Physiol Biophys 6:387-399.

Hall ED, Braughler JM (1986) Role of lipid peroxidation in post-traumatic spinal cord degeneration: a review. Cent Nerv Syst Trauma 3:281-294.

Hall ED, Yonkers PA, Andrus PK, Cox JW, Anderson DK (1992) Biochemistry and pharmacology of lipid antioxidants in acute brain and spinal cord injury. Journal of Neurotrauma 9(suppl 2):S425-S442.

Hamada Y, Ikata T, Katoh S, Tsuchiya K, Niwa M, Tsutsumishita Y, Fukuzawa K (1996) Roles of nitric oxide in compression injury of rat spinal cord. Free Radic Biol Med 20:1-9.

Han WK, Sapirstein A, Hung CC, Alessandrini A, Bonventre JV (2003) Cross-talk between cytosolic phospholipase $\mathrm{A}_{2} \alpha\left(\mathrm{cPLA}_{2} \alpha\right)$ and secretory phospholipase $\mathrm{A}_{2}$ $\left(\mathrm{sLA}_{2}\right)$ in hydrogen peroxide-induced arachidonic acid release in murine mesangial cells: $\mathrm{sPLA}_{2}$ regulates $\mathrm{CPLA}_{2} \alpha$ activity that is responsible for arachidonic acid release. J Biol Chem 278:24153-24163.

Hanasaki K, Arita H (1992a) Characterization of a high affinity binding site for pancreatic-type phospholipase A2 in the rat. Its cellular and tissue distribution. J Biol Chem 267:6414-6420.

Hanasaki K, Arita H (1992b) Purification and characterization of a high-affinity binding protein for pancreatic-type phospholipase A2. Biochim Biophys Acta 1127:233241.

Hanasaki K, Arita H (1999) Biological and pathological functions of phospholipase A2 receptor. Arch Biochem Biophys 372:215-223. 
Hanasaki K, Arita H (2002) Phospholipase A2 receptor: a regulator of biological functions of secretory phospholipase A2. Prostaglandins Other Lipid Mediat 6869:71-82.

Hanasaki K, Ono T, Saiga A, Morioka Y, Ikeda M, Kawamoto K, Higashino K, Nakano K, Yamada K, Ishizaka J, Arita H (1999) Purified group X secretory phospohlopases A2 induced prominent release of arachidonic acid from human myeloid leukemia cells. Journal of Biological Chemistry 274:34203-34211.

Hanasaki K, Yokota Y, Ishizaki J, Itoh T, Arita H (1997) Resistance to endotoxic shock in phospholipase A2 receptor-deficient mice. J Biol Chem 272:32792-32797.

Hankey GJ (2007a) Antiplatelet therapy for stroke prevention. Curr Atheroscler Rep 9:312-318.

Hankey GJ (2007b) Clinical update: management of stroke. Lancet 369:1330-1332.

Hansford KA, Reid RC, Clark CI, Tyndall JD, Whitehouse MW, Guthrie T, McGeary RP, Schafer K, Martin JL, Fairlie DP (2003) D-Tyrosine as a chiral precusor to potent inhibitors of human nonpancreatic secretory phospholipase A2 (IIa) with antiinflammatory activity. Chembiochem 4:181-185.

Hayakawa T, Chang MC, Rapoport SI, Appel NM (2001) Selective dopamine receptor stimulation differentially affects $[3 \mathrm{H}]$ arachidonic acid incorporation, a surrogate marker for phospholipase A2-mediated neurotransmitter signal transduction, in a rodent model of Parkinson's disease. J Pharmacol Exp Ther 296:1074-1084.

Hernandez M, Burillo SL, Crespo MS, Nieto ML (1998) Secretory phospholipase A2 activates the cascade of mitogen-activated protein kinases and cytosolic phospholipase A2 in the human astrocytoma cell line 1321N1. J Biol Chem 273:606-612.

Hidi R, Vargaftig BB, Touqui L (1993) Increased synthesis and secretion of a 14-kDa phospholipase A2 by guinea pig alveolar macrophages. Dissociation from arachidonic acid liberation and modulation by dexamethasone. J Immunol 151:5613-5623.

Ho IC, Arm JP, Bingham CO, 3rd, Choi A, Austen KF, Glimcher LH (2001) A novel group of phospholipase A2s preferentially expressed in type 2 helper T cells. J Biol Chem 276:18321-18326.

Hoffmann C (2000) COX-2 in brain and spinal cord implications for therapeutic use. Curr Med Chem 7:1113-1120.

Homayoun P, Parkins NE, Soblosky J, Carey ME, Rodriguez de Turco EB, Bazan NG (2000) Cortical impact injury in rats promotes a rapid and sustained increase in polyunsaturated free fatty acids and diacylglycerols. Neurochem Res 25:269-276.

Homayoun P, Rodriguez de Turco EB, Parkins NE, Lane DC, Soblosky J, Carey ME, Bazan NG (1997) Delayed phospholipid degradation in rat brain after traumatic brain injury. J Neurochem 69:199-205.

Horigome K, Hayakawa M, Inoue K, Nojima S (1987) Selective release of phospholipase A2 and lysophosphatidylserine-specific lysophospholipase from rat platelets. J Biochem (Tokyo) 101:53-61.

Horrocks LA, Demediuk P, Saunders RD, Dugan L, Clendenon NR, Means ED, Anderson DK (1985) The degradation of phospholipids, formation of metabolites of arachidonic acid, and demyelination following experimental spinal cord injury. Cent Nerv Syst Trauma 2:115-120. 
Hostettler ME, Carlson SL (2002) PAF antagonist treatment reduces pro-inflammatory cytokine mRNA after spinal cord injury. Neuroreport 13:21-24.

Hostettler ME, Knapp PE, Carlson SL (2002) Platelet-activating factor induces cell death in cultured astrocytes and oligodendrocytes: involvement of caspase-3. Glia 38:228-239.

Hoyt KR, Gallagher AJ, Hastings TG, Reynolds IJ (1997) Characterization of hydrogen peroxide toxicity in cultured rat forebrain neurons. Neurochem Res 22:333-340.

Huterer SJ, Tourtellotte WW, Wherrett JR (1995) Alterations in the activity of phospholipases A2 in postmortem white matter from patients with multiple sclerosis. Neurochemical Research 20:1335-1343.

Iannotti C, Ping Zhang Y, Shields CB, Han Y, Burke DA, Xu XM (2004) A neuroprotective role of glial cell line-derived neurotrophic factor following moderate spinal cord contusion injury. Experimental neurology 189:317-332.

Iannotti C, Zhang YP, Shields LB, Han Y, Burke DA, Xu XM, Shields CB (2006) Dural repair reduces connective tissue scar invasion and cystic cavity formation after acute spinal cord laceration injury in adult rats. J Neurotrauma 23:853-865.

Ikeno Y, Konno N, Cheon SH, Bolchi A, Ottonello S, Kitamoto K, Arioka M (2005) Secretory phospholipases A2 induce neurite outgrowth in PC12 cells through lysophosphatidylcholine generation and activation of G2A receptor. J Biol Chem 280:28044-28052.

Ishizaki J, Hanasaki K, Higashino K, Kishino J, Kikuchi N, Ohara O, Arita H (1994) Molecular cloning of pancreatic group I phospholipase A2 receptor. J Biol Chem 269:5897-5904.

Ishizaki J, Suzuki N, Higashino K, Yokota Y, Ono T, Kawamoto K, Fujii N, Arita H, Hanasaki K (1999) Cloning and characterization of novel mouse and human secretory phospholipase A(2)s. J Biol Chem 274:24973-24979.

Ivandic B, Castellani LW, Wang XP, Qiao JH, Mehrabian M, Navab M, Fogelman AM, Grass DS, Swanson ME, de Beer MC, de Beer F, Lusis AJ (1999) Role of group II secretory phospholipase A2 in atherosclerosis: 1 . Increased atherogenesis and altered lipoproteins in transgenic mice expressing group IIa phospholipase A2. Arterioscler Thromb Vasc Biol 19:1284-1290.

Jacobs TP, Shohami E, Baze W, Burgard E, Gunderson C, Hallenbeck J, Feuerstein G (1987) Thromboxane and 5-HETE increase after experimental spinal cord injury in rabbits. Cent Nerv Syst Trauma 4:95-118.

Janzen J, Bersch U, Pietsch-Breitfeld B, Pressler H, Michel D, Bultmann B (2001) Urinary bladder biopsies in spinal cord injured patients. Spinal Cord 39:568-570.

Jasmin L, Janni G, Moallem TM, Lappi DA, Ohara PT (2000) Schwann cells are removed from the spinal cord after effecting recovery from paraplegia. Journal of Neuroscience 20:9215-9223.

Jeffery ND, Blakemore WF (1995) Remyelination of mouse spinal cord axons demyelinated by local injection of lysolecithin. J Neurocytol 24:775-781.

Jiang SD, Jiang LS, Dai LY (2006) Mechanisms of osteoporosis in spinal cord injury. Clin Endocrinol (Oxf) 65:555-565.

Jones TB, Hart RP, Popovich PG (2005a) Molecular control of physiological and pathological T-cell recruitment after mouse spinal cord injury. Journal of Neuroscience 25:6576-6583. 
Jones TB, McDaniel EE, Popovich PG (2005b) Inflammatory-mediated injury and repair in the traumatically injured spinal cord. Current Pharmaceutical Design 11:12231236.

Kalyvas A, David S (2004) Cytosolic phospholipase A2 plays a key role in the pathogenesis of multiple sclerosis-like disease. Neuron 41:323-335.

Kanemasa T, Hanasaki K, Arita H (1992) Migration of vascular smooth muscle cells by phospholipase A2 via specific binding sites. Biochim Biophys Acta 1125:210214.

Kanfer JN, Sorrentino G, Sitar DS (1998) Phospholipases as mediators of amyloid beta peptide neurotoxicity: an early event contributing to neurodegeneration characteristic of Alzheimer's disease. Neurosci Lett 257:93-96.

Kennedy BP, Payette P, Mudgett J, Vadas P, Pruzanski W, Kwan M, Tang C, Rancourt DE, Cromlish WA (1995) A natural disruption of the secretory group II phospholipase A2 gene in inbred mouse strains. J Biol Chem 270:22378-22385.

Keuter M, Dharmana E, Kullberg BJ, Schalkwijk C, Gasem MH, Seuren L, Djokomoeljanto R, Dolmans WM, van den Bosch H, van der Meer JW (1995) Phospholipase A2 is a circulating mediator in typhoid fever. J Infect Dis 172:305308.

Kigerl KA, McGaughy VM, Popovich PG (2006) Comparative analysis of lesion development and intraspinal inflammation in four strains of mice following spinal contusion injury. J Comp Neurol 494:578-594.

Kim DK, Fukuda T, Thompson BT, Cockrill B, Hales C, Bonventre JV (1995) Bronchoalveolar lavage fluid phospholipase A2 activities are increased in human adult respiratory distress syndrome. Am J Physiol 269:L109-118.

Kim KP, Rafter JD, Bittova L, Han SK, Snitko Y, Munoz NM, Leff AR, Cho W (2001) Mechanism of human group V phospholipase A2 (PLA2)-induced leukotriene biosynthesis in human neutrophils. A potential role of heparan sulfate binding in PLA2 internalization and degradation. J Biol Chem 276:11126-11134.

Kini RM, Evans HJ (1987) Structure-function relationships of phospholipases. The anticoagulant region of phospholipases A2. J Biol Chem 262:14402-14407.

Kinoshita E, Handa N, Hanada K, Kajiyama G, Sugiyama M (1997) Activation of MAP kinase cascade induced by human pancreatic phospholipase A2 in a human pancreatic cancer cell line. FEBS Lett 407:343-346.

Kisseberth WC, Brettingen NT, Lohse JK, Sandgren EP (1999) Ubiquitous expression of marker transgenes in mice and rats. Dev Biol 214:128-138.

Kistner A, Gossen M, Zimmermann F, Jerecic J, Ullmer C, Lubbert H, Bujard H (1996) Doxycycline-mediated quantitative and tissue-specific control of gene expression in transgenic mice. Proc Natl Acad Sci U S A 93:10933-10938.

Klein J (2000) Membrane breakdown in acute and chronic neurodegeneration: focus on choline-containing phospholipids. J Neural Transm 107:1027-1063.

Kline AE, Massucci JL, Ma X, Zafonte RD, Dixon CE (2004) Bromocriptine reduces lipid peroxidation and enhances spatial learning and hippocampal neuron survival in a rodent model of focal brain trauma. J Neurotrauma 21:1712-1722.

Klivenyi P, Beal MF, Ferrante RJ, Andreassen OA, Wermer M, Chin MR, Bonventre JV (1998) Mice deficient in group IV cytosolic phospholipase A2 are resistant to MPTP neurotoxicity. Journal of Neurochemistry 71:2634-2637. 
Kolko M, Bruhn J, Schweitz H, Qar J, Lazdunski M, Lambeau G, Bazan NG, Diemer NH (1999) Secretory phospohlopases A2 potentiates glutamate-induced rate striatal neuronal cell death in vivo. Neuroscience Letter 274:167-170.

Kolko M, Christoffersen NR, Barreiro SG, Bazan NG (2004) Expression and location of mRNAs encoding multiple forms of secretory phospholipase A2 in the rat retina. Journal of Neuroscience Research 77:517-524.

Kolko M, Christoffersen NR, Barreiro SG, Miller ML, Pizza AJ, Bazan NG (2006) Characterization and location of secretory phospholipase A2 groups IIE, V, and X in the rat brain. Journal of neuroscience research 83:874-882.

Kolko M, Christoffersen NR, Varoqui H, Bazan NG (2005) Expression and induction of secretory phospholipase A2 group IB in brain. Cell Mol Neurobiol 25:1107-1122.

Kolko M, de Turco EB, Diemer NH, Bazan NG (2002) Secretory phospholipase A2mediated neuronal cell death involves glutamate ionotropic receptors. Neuroreport 13:1963-1966.

Kolko M, DeCoster MA, Rodriguez de Turco EB, Bazan NG (1996) Synergy by secretory phospohlopases A2 and glutamate on inducing cell death and sustained arachidonic acid metabolic changes in primary cortical neuronal cultures. Journal of Biological Chemistry 271:32722-32728.

Kolko M, Rodriguez de Turco EB, Diemer NH, Bazan NG (2003) Neuronal damage by secretory phospholipase A2: modulation by cytosolic phospholipase A2, plateletactivating factor, and cyclooxygenase- 2 in neuronal cells in culture. Neurosci Lett 338:164-168.

Komada M, Kudo I, Mizushima H, Kitamura N, Inoue K (1989) Structure of cDNA coding for rat platelet phospholipase A2. J Biochem (Tokyo) 106:545-547.

Kornecki E, Ehrlich YH (1988) Neuroregulatory and neuropathological actions of the ether-phospholipid platelet-activating factor. Science 240:1792-1794.

Kramer RM, Hession C, Johansen B, Hayes G, McGray P, Chow EP, Tizard R, Pepinsky RB (1989) Structure and properties of a human non-pancreatic phospholipase A2. Journal of Biological Chemistry 264:5768-5775.

Kudo I, Murakami M (2002) Phospholipase A2 enzymes. Prostaglandins \& Other Lipid Mediators 68-69:3-58.

Kuwata H, Nakatani Y, Murakami M, Kudo I (1998) Cytosolic phospholipase A2 is required for cytokine-induced expression of type IIA secretory phospholipase A2 that mediates optimal cyclooxygenase-2-dependent delayed prostaglandin E2 generation in rat 3Y1 fibroblasts. J Biol Chem 273:1733-1740.

Kuwata H, Nonaka T, Murakami M, Kudo I (2005) Search of factors that intermediate cytokine-induced group IIA phospholipase A2 expression through the cytosolic phospholipase A2- and 12/15-lipoxygenase-dependent pathway. J Biol Chem 280:25830-25839.

Kuwata H, Yamamoto S, Miyazaki Y, Shimbara S, Nakatani Y, Suzuki H, Ueda N, Murakami M, Kudo I (2000) Studies on a mechanism by which cytosolic phospholipase A2 regulates the expression and function of type IIA secretory phospholipase A2. J Immunol 165:4024-4031.

Laine VJ, Grass DS, Nevalainen TJ (2000) Resistance of transgenic mice expressing human group II phospholipase A2 to Escherichia coli infection. Infect Immun 68:87-92. 
Laine VJ, Gronroos JM, Nevalainen TJ (1996) Serum phospholipase A2 in patients after splenectomy. Eur J Clin Chem Clin Biochem 34:419-422.

Lambeau G, Ancian P, Barhanin J, Lazdunski M (1994) Cloning and expression of a membrane receptor for secretory phospholipases A2. J Biol Chem 269:15751578 .

Lambeau G, Lazdunski M (1999) Receptors for a growing family of secreted phospholipases A2. Trends in Pharmacological Sciences 20:162-170.

Lauber K, Bohn E, Krober SM, Xiao YJ, Blumenthal SG, Lindemann RK, Marini P, Wiedig C, Zobywalski A, Baksh S, Xu Y, Autenrieth IB, Schulze-Osthoff K, Belka C, Stuhler G, Wesselborg S (2003) Apoptotic cells induce migration of phagocytes via caspase-3-mediated release of a lipid attraction signal. Cell 113:717-730.

Lauritzen I, Heurteaux C, Lazdunski M (1994) Expression of group II phospohlopases A2 in rat brain after severe farebrain ischemia and in endotoxic shock. Brain Reseach 651:353-356.

Layton ME, Pazdernik TL, Samson FE (1997) Cerebral penetration injury leads to H2O2 generation in microdialysis samples. Neurosci Lett 236:63-66.

Leadbeater WE, Gonzalez AM, Logaras N, Berry M, Turnbull JE, Logan A (2006) Intracellular trafficking in neurones and glia of fibroblast growth factor-2, fibroblast growth factor receptor 1 and heparan sulphate proteoglycans in the injured adult rat cerebral cortex. J Neurochem 96:1189-1200.

Lee YB, Yune TY, Baik SY, Shin YH, Du S, Rhim H, Lee EB, Kim YC, Shin ML, Markelonis GJ, Oh TH (2000) Role of tumor necrosis factor-a in neuronal and glial apoptosis after spinal cord injury. Experimental Neurology 166:190-195.

Leitinger N, Watson AD, Hama SY, Ivandic B, Qiao JH, Huber J, Faull KF, Grass DS, Navab M, Fogelman AM, de Beer FC, Lusis AJ, Berliner JA (1999) Role of group II secretory phospholipase A2 in atherosclerosis: 2. Potential involvement of biologically active oxidized phospholipids. Arterioscler Thromb Vasc Biol 19:1291-1298.

Leung PY, Johnson CS, Wrathall JR (2007) Comparison of the effects of complete and incomplete spinal cord injury on lower urinary tract function as evaluated in unanesthetized rats. Exp Neurol 208:80-91.

Lev N, Ickowicz D, Barhum Y, Blondheim N, Melamed E, Offen D (2006) Experimental encephalomyelitis induces changes in DJ-1: implications for oxidative stress in multiple sclerosis. Antioxid Redox Signal 8:1987-1995.

Li W, Xia J, Sun GY (1999) Cytokine induction of iNOS and sPLA2 in immortalized astrocytes (DITNC): response to genistein and pyrrolidine dithiocarbamate. J Interferon Cytokine Res 19:121-127.

Li Y, Maher P, Schubert D (1997) A role for 12-lipoxygenase in nerve cell death caused by glutathione depletion. Neuron 19:453-463.

Lim CS, Lee JC, Kim SD, Chang DJ, Kaang BK (2002) Hydrogen peroxide-induced cell death in cultured Aplysia sensory neurons. Brain Res 941:137-145.

Lin TN, Wang Q, Simonyi A, Chen JJ, Cheung WM, He YY, Xu J, Sun AY, Hsu CY, Sun GY (2004) Induction of secretory phospholipase A2 in reactive astrocytes in response to transient focal cerebral ischemia in the rat brain. J Neurochem 90:637-645. 
Lindsberg PJ, Yue TL, Frerichs KU, Hallenbeck JM, Feuerstein G (1990) Evidence for platelet-activating factor as a novel mediator in experimental stroke in rabbits. Stroke 21:1452-1457.

Liu D, Li L, Augustus L (2001) Prostaglandin release by spinal cord injury mediates production of hydroxyl radical, malondialdehyde and cell death: a site of the neuroprotective action of methylprednisolone. J Neurochem 77:1036-1047.

Liu D, Liu J, Sun D, Alcock NW, Wen J (2003) Spinal cord injury increases iron levels: catalytic production of hydroxyl radicals. Free Radic Biol Med 34:64-71.

Liu D, Liu J, Sun D, Wen J (2004a) The time course of hydroxyl radical formation following spinal cord injury: the possible role of the iron-catalyzed Haber-Weiss reaction. J Neurotrauma 21:805-816.

Liu D, Liu J, Wen J (1999a) Elevation of hydrogen peroxide after spinal cord injury detected by using the Fenton reaction. Free Radic Biol Med 27:478-482.

Liu D, Xu GY, Pan E, McAdoo DJ (1999b) Neurotoxicity of glutamate at the concentration released upon spinal cord injury. Neuroscience 93:1383-1389.

Liu N, Han S, Lu PH, Xu XM (2004b) Upregulation of annexins I, II, and V after traumatic spinal cord injury in adult rats. Journal of neuroscience research 77:391401.

Liu NK, Xu XM (2006) b-tubulin is a more suitable internal control than b-actin in western blot analysis of spinal cord tissues after traumatic injury. J Neurotrauma 23:1794-1801.

Liu NK, Zhang YP, Han S, Pei J, Xu LY, Lu PH, Shields CB, Xu XM (2007) Annexin A1 reduces inflammatory reaction and tissue damage through inhibition of phospholipase A2 activation in adult rats following spinal cord injury. $\mathrm{J}$ Neuropathol Exp Neurol 66:932-943.

Liu NK, Zhang YP, Titsworth WL, Jiang X, Han S, Lu PH, Shields CB, Xu XM (2006) A Novel Role of Phospholipase A2 in Mediating Spinal Cord Secondary Injury. Anal Neurology 59:606-619.

Liu XZ, Xu XM, Hu R, Du C, Zhang SX, McDonald JW, Dong HX, Wu YJ, Fan GS, Jacquin MF, Hsu CY, Choi DW (1997) Neuronal and glial apoptosis after traumatic spinal cord injury. Journal of Neuroscience 17:5395-5406.

Lu XR, Ong WY, Halliwell B (2001) The phospholipase A2 inhibitor quinacrine prevents increased immunoreactivity to cytoplasmic phospholipase A2 (cPLA2) and hydroxynonenal (HNE) in neurons of the lateral septum following fimbria-fornix transection. Exp Brain Res 138:500-508.

Lucas KK, Svensson CI, Hua XY, Yaksh TL, Dennis EA (2005) Spinal phospholipase A2 in inflammatory hyperalgesia: role of group IVA cPLA2. Br J Pharmacol 144:940-952.

Lukacova N, Halat G, Chavko M, Marsala J (1996) Ischemia-reperfusion injury in the spinal cord of rabbits strongly enhances lipid peroxidation and modifies phospholipid profiles. Neurochem Res 21:869-873.

MacPhee M, Chepenik KP, Liddell RA, Nelson KK, Siracusa LD, Buchberg AM (1995) The secretory phospholipase A2 gene is a candidate for the Mom1 locus, a major modifier of ApcMin-induced intestinal neoplasia. Cell 81:957-966.

Maimoun L, Fattal C, Micallef JP, Peruchon E, Rabischong P (2006) Bone loss in spinal cord-injured patients: from physiopathology to therapy. Spinal Cord 44:203-210. 
Makela A, Sternby B, Kuusi T, Puolakkainen P, Schroder T (1990) Phospholipase A2 activity and concentration in several body fluids in patients with acute pancreatitis. Scand J Gastroenterol 25:944-950.

Marusic S, Leach MW, Pelker JW, Azoitei ML, Uozumi N, Cui J, Shen MW, DeClercq CM, Miyashiro JS, Carito BA, Thakker P, Simmons DL, Leonard JP, Shimizu T, Clark JD (2005) Cytosolic phospholipase $\mathrm{A}_{2} \alpha$-deficient mice are resistant to experimental autoimmune encephalomyelitis. J Exp Med 202:841-851.

Massaad C, Paradon M, Jacques C, Salvat C, Bereziat G, Berenbaum F, Olivier JL (2000) Induction of secreted type IIA phospholipase A2 gene transcription by interleukin-1beta. Role of C/EBP factors. J Biol Chem 275:22686-22694.

Masuda S, Yamamoto K, Hirabayashi T, Ishikawa Y, Ishii T, Kudo I, Murakami M (2008) Human group III secreted phospholipase A2 promotes neuronal outgrowth and survival. Biochem J 409:429-438.

Matsuzawa A, Murakami M, Atsumi G, Imai K, Prados P, Inoue K, Kudo I (1996) Release of secretory phospholipase A2 from rat neuronal cells and its possible function in the regulation of catecholamine secretion. Biochem J 318 ( Pt 2):701709.

Mattson MP (2005) Energy intake, meal frequency, and health: a neurobiological perspective. Annu Rev Nutr 25:237-260.

McAdoo DJ, Xu GY, Robak G, Hughes MG (1999) Changes in amino acid concentrations over time and space around an impact injury and their diffusion through the rat spinal cord. Exp Neurol 159:538-544.

McIntosh TK, Saatman KE, Raghupathi R, Graham DI, Smith DH, Lee VM, Trojanowski JQ (1998) The Dorothy Russell Memorial Lecture. The molecular and cellular sequelae of experimental traumatic brain injury: pathogenetic mechanisms. Neuropathol Appl Neurobiol 24:251-267.

McKenna JE, Prusky GT, Whishaw IQ (2000) Cervical motoneuron topography reflects the proximodistal organization of muscles and movements of the rat forelimb: a retrograde carbocyanine dye analysis. J Comp Neurol 419:286-296.

McTigue DM, Wei P, Stokes BT (2001) Proliferation of NG2-positive cells and altered oligodendrocyte numbers in the contused rat spinal cord. Journal of Neuroscience 21:3392-3400.

Meyer TN, Gloy J, Hug MJ, Greger R, Schollmeyer P, Pavenstadt H (1996) Hydrogen peroxide increases the intracellular calcium activity in rat mesangial cells in primary culture. Kidney Int 49:388-395.

Michel RP, Cruz-Orive LM (1988) Application of the Cavalieri principle and vertical sections method to lung: estimation of volume and pleural surface area. Journal of Microscopy 150:117-136.

Minami T, Tojo H, Shinomura Y, Matsuzawa Y, Okamoto M (1994) Increased group II phospholipase A2 in colonic mucosa of patients with Crohn's disease and ulcerative colitis. Gut 35:1593-1598.

Mitsuhashi T, Ikata T, Morimoto K, Tonai T, Katoh S (1994) Increased production of eicosanoids, TXA2, PGI2 and LTC4 in experimental spinal cord injuries. Paraplegia 32:524-530.

Molloy GY, Rattray M, Williams RJ (1998) Genes encoding multiple forms of phospholipase A2 are expressed in rat brain. Neurosci Lett 258:139-142. 
Montoya CP, Astell S, Dunnett SB (1990) Effects of nigral and striatal grafts on skilled forelimb use in the rat. Prog Brain Res 82:459-466.

Montoya CP, Campbell-Hope LJ, Pemberton KD, Dunnett SB (1991) The "staircase test": a measure of independent forelimb reaching and grasping abilities in rats. $\mathrm{J}$ Neurosci Methods 36:219-228.

Morell P, (1984) Myelin. Plenum Press, New York.

Morioka N, Takeda K, Kumagai K, Hanada T, Ikoma K, Hide I, Inoue A, Nakata Y (2002) Interleukin-1b-induced substance $P$ release from rat cultured primary afferent neurons driven by two phospholipase A2 enzymes: secretory type IIA and cytosolic type IV. J Neurochem 80:989-997.

Morioka Y, Ikeda M, Saiga A, Fujii N, Ishimoto Y, Arita H, Hanasaki K (2000a) Potential role of group X secretory phospholipase A(2) in cyclooxygenase-2dependent PGE(2) formation during colon tumorigenesis. FEBS Lett 487:262266.

Morioka Y, Saiga A, Yokota Y, Suzuki N, Ikeda M, Ono T, Nakano K, Fujii N, Ishizaki J, Arita H, Hanasaki K (2000b) Mouse group X secretory phospholipase A2 induces a potent release of arachidonic acid from spleen cells and acts as a ligand for the phospholipase A2 receptor. Arch Biochem Biophys 381:31-42.

Moses GS, Jensen MD, Lue LF, Walker DG, Sun AY, Simonyi A, Sun GY (2006) Secretory PLA2-IIA: a new inflammatory factor for Alzheimer's disease. Journal of neuroinflammation [electronic resource] 3:28.

Mounier CM, Ghomashchi F, Lindsay MR, James S, Singer AG, Parton RG, Gelb MH (2004) Arachidonic acid release from mammalian cells transfected with human groups IIA and $\mathrm{X}$ secreted phospholipase $\mathrm{A}(2)$ occurs predominantly during the secretory process and with the involvement of cytosolic phospholipase $\mathrm{A}(2)$ alpha. J Biol Chem 279:25024-25038.

Mronga T, Stahnke T, Goldbaum O, Richter-Landsberg C (2004) Mitochondrial pathway is involved in hydrogen-peroxide-induced apoptotic cell death of oligodendrocytes. Glia 46:446-455.

Muhl H, Geiger T, Pignat W, Marki F, van den Bosch H, Vosbeck K, Pfeilschifter J (1991) PDGF suppresses the activation of group II phospholipase A2 gene expression by interleukin 1 and forskolin in mesangial cells. FEBS Lett 291:249252.

Murakami M, Kambe T, Shimbara S, Higasgino K, Hansaki K, Arita H, Horiguchi M, Arita M, Arai H, Inoue K, Kudo I (1999a) Different functional aspects of the group II subfamily (type IIA and V) and type X secretory phospohlopases A2s in regulating arachidonic acid release and prostaglandin generation. Implications of cyclooxygenase-2 induction and phospholipid scramblase-mediated cellular membrane perturbation. Journal of Biological Chemistry 274:31435-31444.

Murakami M, Kambe T, Shimbara S, Yamamoto S, Kuwata H, Kudo I (1999b) Functional association of type IIA secretory phospholipase A2 with the glycosylphosphatidylinositol-anchored heparan sulfate proteoglycan in the cyclooxygenase-2-mediated delayed prostanoid-biosynthetic pathway. The Journal of biological chemistry 274:29927-29936.

Murakami M, Koduri RS, Enomoto A, Shimbara S, Seki M, Yoshihara K, Singer A, Valentin E, Ghomashchi F, Lambeau G, Gelb MH, Kudo I (2001) Distinct 
arachidonate-releasing functions of mammalian secreted phospholipase A2s in human embryonic kidney 293 and rat mastocytoma RBL-2H3 cells through heparan sulfate shuttling and external plasma membrane mechanisms. J Biol Chem 276:10083-10096.

Murakami M, Kudo I (2001) Diversity and regulatory functions of mammalian secretory phospholipase A2s. Adv Immunol 77:163-194.

Murakami M, Kudo I, Inoue K (1993) Molecular nature of phospholipases A2 involved in prostaglandin I2 synthesis in human umbilical vein endothelial cells. Possible participation of cytosolic and extracellular type II phospholipases A2. J Biol Chem 268:839-844.

Murakami M, Kudo I, Suwa Y, Inoue K (1992) Release of 14-kDa group-II phospholipase A2 from activated mast cells and its possible involvement in the regulation of the degranulation process. Eur J Biochem 209:257-265.

Murakami M, Nakatani Y, Atsumi G, Inoue K, Kudo I (1997) Regulatory functions of phospholipase A2. Critical Reviews in Immunology 17:225-283.

Murakami M, Nakatani Y, Kudo I (1996) Type II secretory phospholipase $A_{2}$ associated with cell surfaces via $\mathrm{C}$-terminal heparin-binding lysine residues augments stimulus-initiated delayed prostaglandin generation. J Biol Chem 271:3004130051.

Murakami M, Shimbara S, Kambe T, Kuwata H, Winstead MV, Tischfield JA, Kudo I (1998) The functions of five distinct mammalian phospholipase A2s in regulating arachidonic acid release. Type IIa and type V secretory phospholipase A2s are functionally redundant and act in concert with cytosolic phospholipase A2. Journal of Biological Chemistry 273:14411-14423.

Murakami M, Yoshihara K, Shimbara S, Lambeau G, Gelb MH, Singer AG, Sawada M, Inagaki N, Nagai H, Ishihara M, Ishikawa Y, Ishii T, Kudo I (2002a) Cellular arachidonate-releasing function and inflammation-associated expression of group IIF secretory phospholipase A2. J Biol Chem 277:19145-19155.

Murakami M, Yoshihara K, Shimbara S, Lambeau G, Singer A, Gelb MH, Sawada M, Inagaki N, Nagai H, Kudo I (2002b) Arachidonate release and eicosanoid generation by group IIE phospholipase A2. Biochemical and biophysical research communications 292:689-696.

Muralikrishna Adibhatla R, Hatcher JF (2006) Phospholipase A2, reactive oxygen species, and lipid peroxidation in cerebral ischemia. Free Radic Biol Med 40:376387.

Nakano S, Kogure K, Abe K, Yae T (1990) Ischemia-induced alterations in lipid metabolism of the gerbil cerebral cortex: I. Changes in free fatty acid liberation. J Neurochem 54:1911-1916.

Nanda BL, Nataraju A, Rajesh R, Rangappa KS, Shekar MA, Vishwanath BS (2007) PLA2 mediated arachidonate free radicals: PLA2 inhibition and neutralization of free radicals by anti-oxidants--a new role as anti-inflammatory molecule. Curr Top Med Chem 7:765-777.

Narita K, Kubota M, Nakane M, Kitahara S, Nakagomi T, Tamura A, Hisaki H, Shimasaki H, Ueta N (2000) Therapeutic time window in the penumbra during permanent focal ischemia in rats: changes of free fatty acids and glycerophospholipids. Neurol Res 22:393-400. 
Nethery D, Stofan D, Callahan L, DiMarco A, Supinski G (1999) Formation of reactive oxygen species by the contracting diaphragm is PLA(2) dependent. J Appl Physiol 87:792-800.

Neuzil J, Upston JM, Witting PK, Scott KF, Stocker R (1998) Secretory phospholipase A2 and lipoprotein lipase enhance 15-lipoxygenase-induced enzymic and nonenzymic lipid peroxidation in low-density lipoproteins. Biochemistry 37:9203-9210.

Nevalainen TJ, Haapamèaki MM, Grèonroos JM (2000) Roles of secretory phospholipases A(2) in inflammatory diseases and trauma. Biochimica et biophysica acta 1488:83-90.

Nottingham S, Knapp P, Springer J (2002) FK506 treatment inhibits caspase-3 activation and promotes oligodendroglial survival following traumatic spinal cord injury. Experimental Neurology 177:242-251.

NSCISC, 2008. SPINAL CORD INJURY: Facts and Figures at a Glance. In: National Spinal Cord Injury Statistical Center), vol. 2008. no. 05/06/2008. University of Alabama at Birmingham, Birmingham, AL.

O'Regan MH, Alix S, Woodbury DJ (1996) Phospholipase A2-evoked destabilization of planar lipid membranes. Neurosci Lett 202:201-203.

O'Regan MH, Smith-Babour M, Perkins LM, Phillis JW (1995a) A possible role for phospohlopases in the release of neurotransmitter amino acids from ischemic rat cerebral cortex. Neuroscience Letter 185:191-194.

O'Regan MH, Smith-Barbour M, Perkins LM, Phillis JW (1995b) A possible role for phospholipases in the release of neurotransmitter amino acids from ischemic rat cerebral cortex. Neurosci Lett 185:191-194.

Ohara O, Ishizaki J, Arita H (1995) Structure and function of phospholipase A2 receptor. Prog Lipid Res 34:117-138.

Oka S, Arita H (1991) Inflammatory factors stimulate expression of group II phospholipase A2 in rat cultured astrocytes. Two distinct pathways of the gene expression. Journal of Biological Chemistry 266:9956-9960.

Ong WY, Lu XR, Ong BK, Horrocks LA, Farooqui AA, Lim SK (2003) Quinacrine abolishes increases in cytoplasmic phospholipase A2 mRNA levels in the rat hippocampus after kainate-induced neuronal injury. Exp Brain Res 148:521-524.

Onifer SM, Nunn CD, Decker JA, Payne BN, Wagoner MR, Puckett AH, Massey JM, Armstrong J, Kaddumi EG, Fentress KG, Wells MJ, West RM, Calloway CC, Schnell JT, Whitaker CM, Burke DA, Hubscher CH (2007) Loss and spontaneous recovery of forelimb evoked potentials in both the adult rat cuneate nucleus and somatosensory cortex following contusive cervical spinal cord injury. Exp Neurol 207:238-247.

Onifer SM, Rodriguez JF, Santiago DI, Benitez JC, Kim DT, Brunschwig J-PR, Pacheco JT, Perrone JV, Llorente O, Hesse DH, Martinez-Arizala A (1997) Cervical Spinal Cord Injury in the Adult Rat: Assessment of Forelimb Dysfunction. Restorative Neurology and Neuroscience 11:211-223.

Onifer SM, Zhang YP, Burke DA, Brooks DL, Decker JA, McClure NJ, Floyd AR, Hall J, Proffitt BL, Shields CB, Magnuson DS (2005) Adult rat forelimb dysfunction after dorsal cervical spinal cord injury. Experimental Neurology 192:25-38. 
Oorschot DE (1994) Are you using neuronal densities, synaptic densities or neurochemical densities as your definitive data? There is a better way to go. Progress in Neurobiology 44:233-247.

Otamiri T, Lindahl M, Tagesson C (1988) Phospholipase A2 inhibition prevents mucosal damage associated with small intestinal ischaemia in rats. Gut 29:489-494.

Ousman SS, David S (2000) Lysophosphatidylcholine induces rapid recruitment and activation of macrophages in the adult mouse spinal cord. Glia 30:92-104.

Ousman SS, David S (2001) MIP-1alpha, MCP-1, GM-CSF, and TNF-alpha control the immune cell response that mediates rapid phagocytosis of myelin from the adult mouse spinal cord. J Neurosci 21:4649-4656.

Owada Y, Tominaga T, Yoshimoto T, Kondo H (1994) Molecular cloning of rat cDNA for cytosolic phospholipase A2 and the increased gene expression in the dentate gyrus following transient forebrain ischemia. Brain Res Mol Brain Res 25:364368.

Panter SS, Yum SW, Faden AI (1990) Alteration in extracellular amino acids after traumatic spinal cord injury. Ann Neurol 27:96-99.

Park E, Velumian AA, Fehlings MG (2004) The role of excitotoxicity in secondary mechanisms of spinal cord injury: a review with an emphasis on the implications for white matter degeneration. J Neurotrauma 21:754-774.

Pearse DD, Pereira FC, Marcillo AE, Bates ML, Berrocal YA, Filbin MT, Bunge MB (2004) cAMP and Schwann cells promote axonal growth and functional recovery after spinal cord injury. Nature Medicine 10:610-616.

Peilot H, Rosengren B, Bondjers G, Hurt-Camejo E (2000) Interferon-g induces secretory group IIA phospholipase A2 in human arterial smooth muscle cells. Involvement of cell differentiation, STAT-3 activation, and modulation by other cytokines. $\mathrm{J}$ Biol Chem 275:22895-22904.

Phillis JW (1996) Cerebroprotective action of the phospholipase inhibitor quinacrine in the ischemia/reperfused gerbil hippocampus. Life Science 58:PL97-PL101.

Phillis JW, O'Regan MH (1996) Mechanisms of glutamate and aspartate release in the ischemic rat cerebral cortex. Brain Res 730:150-164.

Phillis JW, O'Regan MH (2004) A potentially critical role of phospholipases in central nervous system ischemic, traumatic, and neurodegenerative disorders. Brain Res Brain Res Rev 44:13-47.

Pilitsis JG, Coplin WM, O'Regan MH, Wellwood JM, Diaz FG, Fairfax MR, Michael DB, Phillis JW (2003) Measurement of free fatty acids in cerebrospinal fluid from patients with hemorrhagic and ischemic stroke. Brain Res 985:198-201.

Pinto F, Brenner T, Dan P, Krimsky M, Yedgar S (2003) Extracellular phospholipase A2 inhibitors suppress central nervous system inflammation. GLIA 44:275-282.

Piomelli D, Astarita G, Rapaka R (2007) A neuroscientist's guide to lipidomics. Nat Rev Neurosci 8:743-754.

Popovich PG, Guan Z, Wei P, Huitinga I, van Rooijen N, Stokes BT (1999) Depletion of hematogenous macrophages promotes partial hindlimb recovery and neuroanatomical repair after experimental spinal cord injury. Exp Neurol 158:351-365.

Popovich PG, Wei P, Stokes BT (1997) Cellular inflammatory response after spinal cord injury in Sprague-Dawley and Lewis rats. J Comp Neurol 377:443-464. 
Porcellati G, 1983. Phospholipid metabolism in neural membranes. In: Sun, G. Y. et al. (Eds.), Neural Membranes. Humana Press, New York.

Rabchevsky AG, Fugaccia I, Fletcher-Turner A, Blades DA, Mattson MP, Scheff SW (1999) Basic fibroblast growth factor (bFGF) enhances tissue sparing and functional recovery following moderate spinal cord injury. J Neurotrauma 16:817830.

Rabchevsky AG, Fugaccia I, Sullivan PG, Scheff SW (2001) Cyclosporin A treatment following spinal cord injury to the rat: behavioral effects and stereological assessment of tissue sparing. J Neurotrauma 18:513-522.

Rabchevsky AG, Fugaccia I, Turner AF, Blades DA, Mattson MP, Scheff SW (2000) Basic fibroblast growth factor (bFGF) enhances functional recovery following severe spinal cord injury to the rat. Exp Neurol 164:280-291.

Raisman G (2004) Myelin inhibitors: does NO mean GO? Nat Rev Neurosci 5:157-161.

Reddy PH (2006) Amyloid precursor protein-mediated free radicals and oxidative damage: implications for the development and progression of Alzheimer's disease. J Neurochem 96:1-13.

Rego AC, Oliveira CR (2003) Mitochondrial dysfunction and reactive oxygen species in excitotoxicity and apoptosis: implications for the pathogenesis of neurodegenerative diseases. Neurochem Res 28:1563-1574.

Reid RC (2005) Inhibitors of secretory phospholipase A2 group IIA. Curr Med Chem 12:3011-3026.

Resnick DK, Graham SH, Dixon CE, Marion DW (1998) Role of cyclooxygenase 2 in acute spinal cord injury. J Neurotrauma 15:1005-1013.

Resnick DK, Nguyen P, Cechvala CF (2001) Regional and temporal changes in prostaglandin E2 and thromboxane B2 concentrations after spinal cord injury. Spine J 1:432-436.

Richter-Landsberg C, Vollgraf U (1998) Mode of cell injury and death after hydrogen peroxide exposure in cultured oligodendroglia cells. Exp Cell Res 244:218-229.

Rigoni M, Caccin P, Gschmeissner S, Koster G, Postle AD, Rossetto O, Schiavo G, Montecucco C (2005) Equivalent effects of snake PLA2 neurotoxins and lysophospholipid-fatty acid mixtures. Science 310:1678-1680.

Ross BM, Moszczynska A, Erlich J, Kish SJ (1998) Phospholipid-metabolizing enzymes in Alzheimer's disease: increased lysophospholipid acyltransferase activity and decreased phospholipase A2 activity. J Neurochem 70:786-793.

Rouach N, Calvo CF, Duquennoy H, Glowinski J, Giaume C (2004) Hydrogen peroxide increases gap junctional communication and induces astrocyte toxicity: regulation by brain macrophages. Glia 45:28-38.

Saluja I, Song D, O'Regan MH, Phillis JW (1997) Role of phospholipase A2 in the release of free fatty acids during ischemia-reperfusion in the rat cerebral cortex. Neurosci Lett 233:97-100.

Samanta S, Perkinton MS, Morgan M, Williams RJ (1998) Hydrogen peroxide enhances signal-responsive arachidonic acid release from neurons: role of mitogenactivated protein kinase. J Neurochem 70:2082-2090.

Sapirstein A, Bonventre JV (2000) Phospohlopases A2 in ischemic and toxic brain injury. Neurochemistry Research 25:745-753. 
Sawada H, Murakami M, Enomoto A, Shimbara S, Kudo I (1999) Regulation of type V phospholipase A2 expression and function by proinflammatory stimuli. Eur J Biochem 263:826-835.

Schaefers HJ, Haselmann J, Goppelt-Struebe M (1996) Regulation of prostaglandin synthesis in Madin Darby canine kidney cells: role of prostaglandin G/H synthase and secreted phospholipase A2. Biochim Biophys Acta 1300:197-202.

Schalkwijk C, Pfeilschifter J, Marki F, van den Bosch H (1992) Interleukin-1b and forskolin-induced synthesis and secretion of group II phospholipase A2 and prostaglandin E2 in rat mesangial cells is prevented by transforming growth factor b2. J Biol Chem 267:8846-8851.

Schaloske RH, Dennis EA (2006) The phospholipase A2 superfamily and its group numbering system. Biochim Biophys Acta 1761:1246-1259.

Scheff SW, Rabchevsky AG, Fugaccia I, Main JA, Lumpp JE, Jr. (2003) Experimental modeling of spinal cord injury: characterization of a force-defined injury device. $\mathrm{J}$ Neurotrauma 20:179-193.

Schrimsher GW, Reier PJ (1993) Forelimb motor performance following dorsal column, dorsolateral funiculi, or ventrolateral funiculi lesions of the cervical spinal cord in the rat. Exp Neurol 120:264-276.

Schwab JM, Brechtel K, Nguyen TD, Schluesener HJ (2000) Persistent accumulation of cyclooxygenase-1 (COX-1) expressing microglia/macrophages and upregulation by endothelium following spinal cord injury. J Neuroimmunol 111:122-130.

Seilhamer JJ, Pruzanski W, Vadas P, Plant S, Miller JA, Kloss J, Johnson LK (1989) Cloning and recombinant expression of phospholipase $\mathrm{A}_{2}$ present in rheumatoid arthritic synovial fluid. J Biol Chem 264:5335-5338.

Seilhamer JJ, Randall TL, Yamanaka M, Johnson LK (1986) Pancreatic phospholipase $\mathrm{A}_{2}$ : isolation of the human gene and cDNAs from porcine pancreas and human lung. DNA 5:519-527.

Selmaj KW, Raine CS (1988) Tumor necrosis factor mediates myelin and oligodendrocyte damage in vitro. Annals of Neurology 23:339-346.

Shinohara H, Amabe Y, Komatsubara T, Tojo H, Okamoto M, Wakano Y, Ishida H (1992) Group II phospholipase A2 induced by interleukin-1 beta in cultured rat gingival fibroblasts. FEBS Lett 304:69-72.

Shirai Y, Ito M (2004) Specific differential expression of phospholipase A2 subtypes in rat cerebellum. J Neurocytol 33:297-307.

Shohami E, Shapira Y, Yadid G, Reisfeld N, Yedgar S (1989) Brain phospholipase A2 is activated after experimental closed head injury in the rat. J Neurochem 53:15411546.

Singh IN, Sorrentino G, Sitar DS, Kanfer JN (1998) (-)Nicotine inhibits the activations of phospholipases A2 and D by amyloid beta peptide. Brain Res 800:275-281.

Springer JE, Azbill RD, Mark RJ, Begley JG, Waeg G, Mattson MP (1997) 4hydroxynonenal, a lipid peroxidation product, rapidly accumulates following traumatic spinal cord injury and inhibits glutamate uptake. J Neurochem 68:24692476.

Stephenson D, Rash K, Smalstig B, Roberts E, Johnstone E, Sharp J, Panetta J, Little S, Kramer R, Clemens J (1999) Cytosolic phospholipase A2 is induced in reactive glia following different forms of neurodegeneration. Glia 27:110-128. 
Stephenson DT, Lemere CA, Selkoe DJ, Clemens JA (1996) Cytosolic phospholipase A2 (cPLA2) immunoreactivity is elevated in Alzheimer's disease brain. Neurobiology of Disease 3:51-63.

Steward O, Zheng B, Tessier-Lavigne M (2003) False resurrections: distinguishing regenerated from spared axons in the injured central nervous system. J Comp Neurol 459:1-8.

Suga H, Murakami M, Kudo I, Inoue K (1993) Participation in cellular prostaglandin synthesis of type-II phospholipase A2 secreted and anchored on cell-surface heparan sulfate proteoglycan. Eur J Biochem 218:807-813.

Sun GY, Horrocks LA, Farooqui AA (2007) The roles of NADPH oxidase and phospholipases A2 in oxidative and inflammatory responses in neurodegenerative diseases. J Neurochem 103:1-16.

Sun GY, Xu J, Jensen MD, Simonyi A (2004) Phospholipase A2 in the central nervous system: implications for neurodegenerative diseases. Journal of Lipid Research 45:205-213.

Sundstrom E, Mo LL (2002) Mechanisms of glutamate release in the rat spinal cord slices during metabolic inhibition. J Neurotrauma 19:257-266.

Suzuki N, Ishizaki J, Yokota Y, Higashino K, Ono T, Ikeda M, Fujii N, Kawamoto K, Hanasaki K (2000) Structures, enzymatic properties, and expression of novel human and mouse secretory phospholipase A(2)s. The Journal of biological chemistry 275:5785-5793.

Svensson CI, Lucas KK, Hua XY, Powell HC, Dennis EA, Yaksh TL (2005) Spinal phospholipase A2 in inflammatory hyperalgesia: role of the small, secretory phospholipase A2. Neuroscience 133:543-553.

Tabuchi S, Uozumi N, Ishii S, Shimizu Y, Watanabe T, Shimizu T (2003) Mice deficient in cytosolic phospholipase A2 are less susceptible to cerebral ischemia/reperfusion injury. Acta Neurochir Suppl 86:169-172.

Takahashi JL, Giuliani F, Power C, Imai Y, Yong VW (2003) Interleukin-1b promotes oligodendrocyte death through glutamate excitotoxicity. Annals of Neurology 53:588-595.

Takaku K, Sonoshita M, Sasaki N, Uozumi N, Doi Y, Shimizu T, Taketo MM (2000) Suppression of intestinal polyposis in Apc(delta 716) knockout mice by an additional mutation in the cytosolic phospholipase A(2) gene. J Biol Chem 275:34013-34016.

Takami T, Oudega M, Bates ML, Wood PM, Kleitman N, Bunge MB (2002) Schwann cell but not olfactory ensheathing glia transplants improve hindlimb locomotor performance in the moderately contused adult rat thoracic spinal cord. Journal of Neuroscience 22:6670-6681.

Taketo MM, Sonoshita M (2002) Phospolipase A2 and apoptosis. Biochim Biophys Acta 1585:72-76.

Talbot K, Young RA, Jolly-Tornetta C, Lee VM, Trojanowski JQ, Wolf BA (2000) A frontal variant of Alzheimer's disease exhibits decreased calcium-independent phospholipase A2 activity in the prefrontal cortex. Neurochem Int 37:17-31.

Talbott JF, Cao Q, Enzmann GU, Benton RL, Achim V, Cheng XX, Mills MD, Rao MS, Whittemore SR (2006) Schwann cell-like differentiation by adult oligodendrocyte 
precursor cells following engraftment into the demyelinated spinal cord is BMPdependent. Glia 54:147-159.

Talbott JF, Loy DN, Liu Y, Qiu MS, Bunge MB, Rao MS, Whittemore SR (2005) Endogenous Nkx2.2+/Olig2+ oligodendrocyte precursor cells fail to remyelinate the demyelinated adult rat spinal cord in the absence of astrocytes. Experimental Neurology 192:11-24.

Tang X, Davies JE, Davies SJ (2003) Changes in distribution, cell associations, and protein expression levels of NG2, neurocan, phosphacan, brevican, versican V2, and tenascin-C during acute to chronic maturation of spinal cord scar tissue. J Neurosci Res 71:427-444.

Taoka Y, Okajima K, Uchiba M, Murakami K, Kushimoto S, Johno M, Naruo M, Okabe $\mathrm{H}$, Takatsuki K (1997) Role of neutrophils in spinal cord injury in the rat. Neuroscience 79:1177-1182.

Tariq M, Khan HA, Al Moutaery K, Al Deeb S (2001) Protective effect of quinacrine on striatal dopamine levels in 6-OHDA and MPTP models of Parkinsonism in rodents. Brain research bulletin 54:77-82.

Tator CH (1991) Review of experimental spinal cord injury with emphasis on the local and systemic circulatory effects. Neurochirurgie 37:291-302.

Tator CH, Fehlings MG (1991) Review of the secondary injury theory of acute spinal cord trauma with emphasis on vascular mechanisms. J Neurosurg 75:15-26.

Thwin MM, Douni E, Aidinis V, Kollias G, Kodama K, Sato K, Satish RL, Mahendran R, Gopalakrishnakone P (2004) Effect of phospholipase A2 inhibitory peptide on inflammatory arthritis in a TNF transgenic mouse model: a time-course ultrastructural study. Arthritis Res Ther 6:R282-294.

Thwin MM, Ong WY, Fong CW, Sato K, Kodama K, Farooqui AA, Gopalakrishnakone P (2003) Secretory phospholipase A2 activity in the normal and kainate injected rat brain, and inhibition by a peptide derived from python serum. Experimental brain research Experimentelle Hirnforschung Expâerimentation câerâebrale 150:427-433.

Tietge UJ, Maugeais C, Cain W, Grass D, Glick JM, de Beer FC, Rader DJ (2000) Overexpression of secretory phospholipase $\mathrm{A}(2)$ causes rapid catabolism and altered tissue uptake of high density lipoprotein cholesteryl ester and apolipoprotein A-I. J Biol Chem 275:10077-10084.

Titsworth WL, Onifer SM, Liu NK, Xu XM (2007) Focal phospholipases A2 group III injections induce cervical white matter injury and functional deficits with delayed recovery concomitant with Schwann cell remyelination. Exp Neurol 207:150-162.

Toborek M, Malecki A, Garrido R, Mattson MP, Hennig B, Young B (1999) Arachidonic acid-induced oxidative injury to cultured spinal cord neurons. J Neurochem 73:684-692.

Tonai T, Taketani Y, Ueda N, Nishisho T, Ohmoto Y, Sakata Y, Muraguchi M, Wada K, Yamamoto S (1999) Possible involvement of interleukin-1 in cyclooxygenase-2 induction after spinal cord injury in rats. J Neurochem 72:302-309.

Tong W, Hu ZY, Sun GY (1995) Stimulation of group II phospholipase A2 mRNA expression and release in an immortalized astrocyte cell line (DITNC) by LPS, TNF a, and IL-1 b. Interactive effects. Molecular \& Chemical Neuropathology 25:1-17. 
Totoiu MO, Keirstead HS (2005) Spinal cord injury is accompanied by chronic progressive demyelination. Journal of Comparative Neurology 486:373-383.

Touqui L, Alaoui-El-Azher M (2001) Mammalian secreted phospholipases A2 and their pathophysiological significance in inflammatory diseases. Curr Mol Med 1:739754.

Tournier C, Thomas G, Pierre J, Jacquemin C, Pierre M, Saunier B (1997) Mediation by arachidonic acid metabolites of the $\mathrm{H} 2 \mathrm{O} 2$-induced stimulation of mitogenactivated protein kinases (extracellular-signal-regulated kinase and c-Jun NH2terminal kinase). Eur J Biochem 244:587-595.

Uhl W, Buchler M, Nevalainen TJ, Deller A, Beger HG (1990) Serum phospholipase A2 in patients with multiple injuries. J Trauma 30:1285-1290.

Valentin E, Ghomashchi F, Gelb MH, Lazdunski M, Lambeau G (2000a) Novel human secreted phospholipase A(2) with homology to the group III bee venom enzyme. J Biol Chem 275:7492-7496.

Valentin E, Koduri RS, Scimeca JC, Carle G, Gelb MH, Lazdunski M, Lambeau G (1999) Cloning and recombinant expression of a novel mouse-secreted phospholipase A2. J Biol Chem 274:19152-19160.

Valentin E, Singer AG, Ghomashchi F, Lazdunski M, Gelb MH, Lambeau G (2000b) Cloning and recombinant expression of human group IIF-secreted phospholipase A(2). Biochem Biophys Res Commun 279:223-228.

Verheij HM, Slotboom AJ, de Haas GH (1981) Structure and function of phospholipase A2. Rev Physiol Biochem Pharmacol 91:91-203.

Vollgraf U, Wegner M, Richter-Landsberg C (1999) Activation of AP-1 and nuclear factor-kappaB transcription factors is involved in hydrogen peroxide-induced apoptotic cell death of oligodendrocytes. J Neurochem 73:2501-2509.

Wang H, Li J, Follett PL, Zhang Y, Cotanche DA, Jensen FE, Volpe JJ, Rosenberg PA (2004) 12-Lipoxygenase plays a key role in cell death caused by glutathione depletion and arachidonic acid in rat oligodendrocytes. Eur J Neurosci 20:20492058.

Wang XF, Huang LD, Yu PP, Hu JG, Yin L, Wang L, Xu XM, Lu PH (2006) Upregulation of type I interleukin-1 receptor after traumatic spinal cord injury in adult rats. Acta Neuropathol (Berl) 111:220-228.

Wei S, Ong WY, Thwin MM, Fong CW, Farooqui AA, Gopalakrishnakone P, Hong W (2003) Group IIA secretory phospholipase A2 stimulates exocytosis and neurotransmitter release in pheochromocytoma- 12 cells and cultured rat hippocampal neurons. Neuroscience 121:891-898.

Weinrauch Y, Elsbach P, Madsen LM, Foreman A, Weiss J (1996) The potent antiStaphylococcus aureus activity of a sterile rabbit inflammatory fluid is due to a 14-kD phospholipase A2. J Clin Invest 97:250-257.

Wells JE, Hurlbert RJ, Fehlings MG, Yong VW (2003) Neuroprotection by minocycline facilitates significant recovery from spinal cord injury in mice. Brain 126:16281637.

Whittemore ER, Loo DT, Cotman CW (1994) Exposure to hydrogen peroxide induces cell death via apoptosis in cultured rat cortical neurons. Neuroreport 5:1485-1488. 
Wightman PD, Dahlgren ME, Davies P, Bonney RJ (1981) The selective release of phospholipase A2 by resident mouse peritoneal macrophages. Biochem J 200:441-444.

Xia Z, Dickens M, Raingeaud J, Davis RJ, Greenberg ME (1995) Opposing effects of ERK and JNK-p38 MAP kinases on apoptosis. Science 270:1326-1331.

Xiao J, Zao D, Zhen H (1995) [Effects of platelet activating factor and platelet activating factor receptor antagonist on spinal cord blood flow after spinal cord injury]. Zhonghua Wai Ke Za Zhi 33:715-718.

Xiao J, Zhao D, Hou T, Wu K, Zeng H (1998) Synergetic protective effects of combined blockade by two kinds of autolesion mediator receptor on neurological function after cervical cord injury. Chin Med J (Engl) 111:443-446.

Xiao J, Zhao D, Jia L (1996) [Effect of platelet activating factor receptor at spinal cord neurocyte membrane on secondary damage after spinal cord injury]. Zhonghua Yi Xue Za Zhi 76:120-123.

Xu J, Chalimoniuk M, Shu Y, Simonyi A, Sun AY, Gonzalez FA, Weisman GA, Wood WG, Sun GY (2003) Prostaglandin E2 production in astrocytes: regulation by cytokines, extracellular ATP, and oxidative agents. Prostaglandins Leukotrienes \& Essential Fatty Acids 69:437-448.

Xu XM, Chen A, Guenard V, Kleitman N, Bunge MB (1997) Bridging Schwann cell transplants promote axonal regeneration from both the rostral and caudal stumps of transected adult rat spinal cord. J Neurocytol 26:1-16.

Xu XM, Guenard V, Kleitman N, Bunge MB (1995) Axonal regeneration into Schwann cell-seeded guidance channels grafted into transected adult rat spinal cord. J Comp Neurol 351:145-160.

Xu XM, Zhang SX, Li H, Aebischer P, Bunge MB (1999a) Regrowth of axons into the distal spinal cord through a Schwann-cell-seeded mini-channel implanted into hemisected adult rat spinal cord. European Journal of Neuroscience 11:17231740 .

Xu XM, Zhang SX, Li H, Aebischer P, Bunge MB (1999b) Regrowth of axons into the distal spinal cord through a Schwann-cell-seeded mini-channel implanted into hemisected adult rat spinal cord. Eur J Neurosci 11:1723-1740.

$\mathrm{Xu}$ Y, Tao YX (2004) Involvement of the NMDA receptor/nitric oxide signal pathway in platelet-activating factor-induced neurotoxicity. Neuroreport 15:263-266.

Yagami T, Ueda K, Asakura K, Hata S, Kuroda T, Sakaeda T, Kishino J, Sakaguchi G, Itoh N, Hori Y (2002a) Group IB secretory phospholipase A2 induces cell death in the cultured cortical neurons: a possible involvement of its binding sites. Brain Research 949:197-201.

Yagami T, Ueda K, Asakura K, Hata S, Kuroda T, Sakaeda T, Takasu N, Tanaka K, Gemba T, Hori Y (2002b) Human group IIA secretory phospholipase A2 induces neuronal cell death via apoptosis. Molecular Pharmacology 61:114-126.

Yagami T, Ueda K, Asakura K, Hayasaki-Kajiwara Y, Nakazato H, Sakaeda T, Hata S, Kuroda T, Takasu N, Hori Y (2002c) Group IB secretory phospholipase A2 induces neuronal cell death via apoptosis. Journal of Neurochemistry 81:449-461.

Yagami T, Ueda K, Asakura K, Nakazato H, Hata S, Kuroda T, Sakaeda T, Sakaguchi G, Itoh N, Hashimoto Y, Hori Y (2003) Human group IIA secretory phospholipase 
$\mathrm{A} 2$ potentiates $\mathrm{Ca} 2+$ influx through $\mathrm{L}$-type voltage-sensitive $\mathrm{Ca} 2+$ channels in cultured rat cortical neurons. Journal of Neurochemistry 85:749-758.

Yagami T, Ueda K, Sakaeda T, Okamura N, Nakazato H, Kuroda T, Hata S, Sakaguchi G, Itoh N, Hashimoto Y, Fujimoto M (2005) Effects of an endothelin B receptor agonist on secretory phospholipase A2-IIA-induced apoptosis in cortical neurons. Neuropharmacology 48:291-300.

Yokota Y, Notoya M, Higashino K, Ishimoto Y, Nakano K, Arita H, Hanasaki K (2001) Clearance of group $\mathrm{X}$ secretory phospholipase $\mathrm{A}(2)$ via mouse phospholipase A(2) receptor. FEBS Lett 509:250-254.

Yoshida S, Inoh S, Asano T, Sano K, Shimasaki H, Ueta N (1983) Brain free fatty acids, edema, and mortality in gerbils subjected to transient, bilateral ischemia, and effect of barbiturate anesthesia. J Neurochem 40:1278-1286.

Young W (1993) Secondary injury mechanisms in acute spinal cord injury. J Emerg Med 11 Suppl 1:13-22.

Zai LJ, Yoo S, Wrathall JR (2005) Increased growth factor expression and cell proliferation after contusive spinal cord injury. Brain Res 1052:147-155.

Zhang YP, Iannotti C, Shields LB, Han Y, Burke DA, Xu XM, Shields CB (2004) Dural closure, cord approximation, and clot removal: enhancement of tissue sparing in a novel laceration spinal cord injury model. J Neurosurg 100:343-352. 


\section{APPENDIX}

Supplemental Figure 1

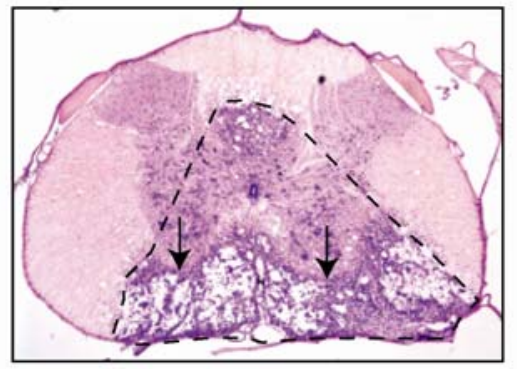


Supplemental Figure 2
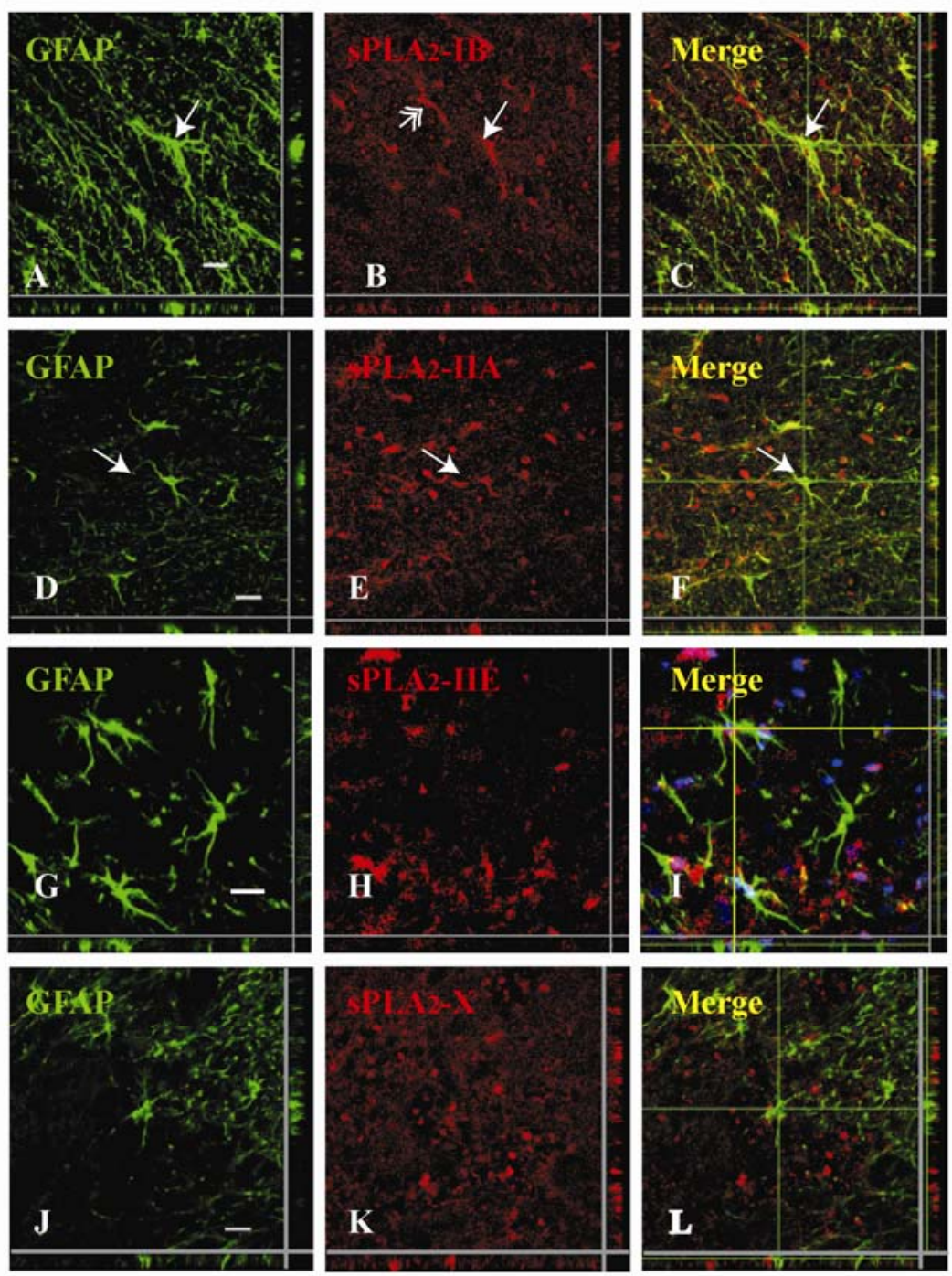
Supplemental Figure 3
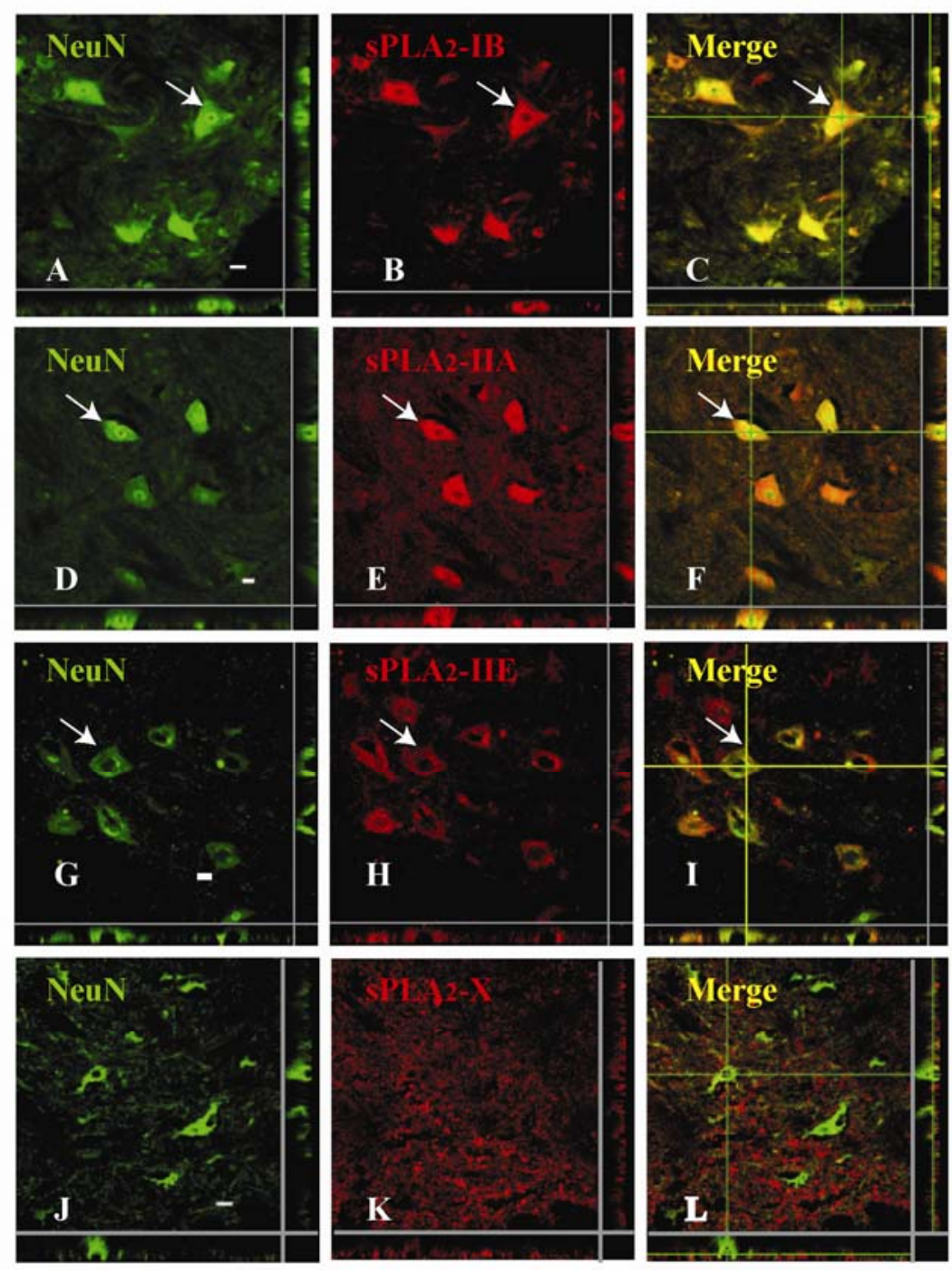
Supplemental Figure 4
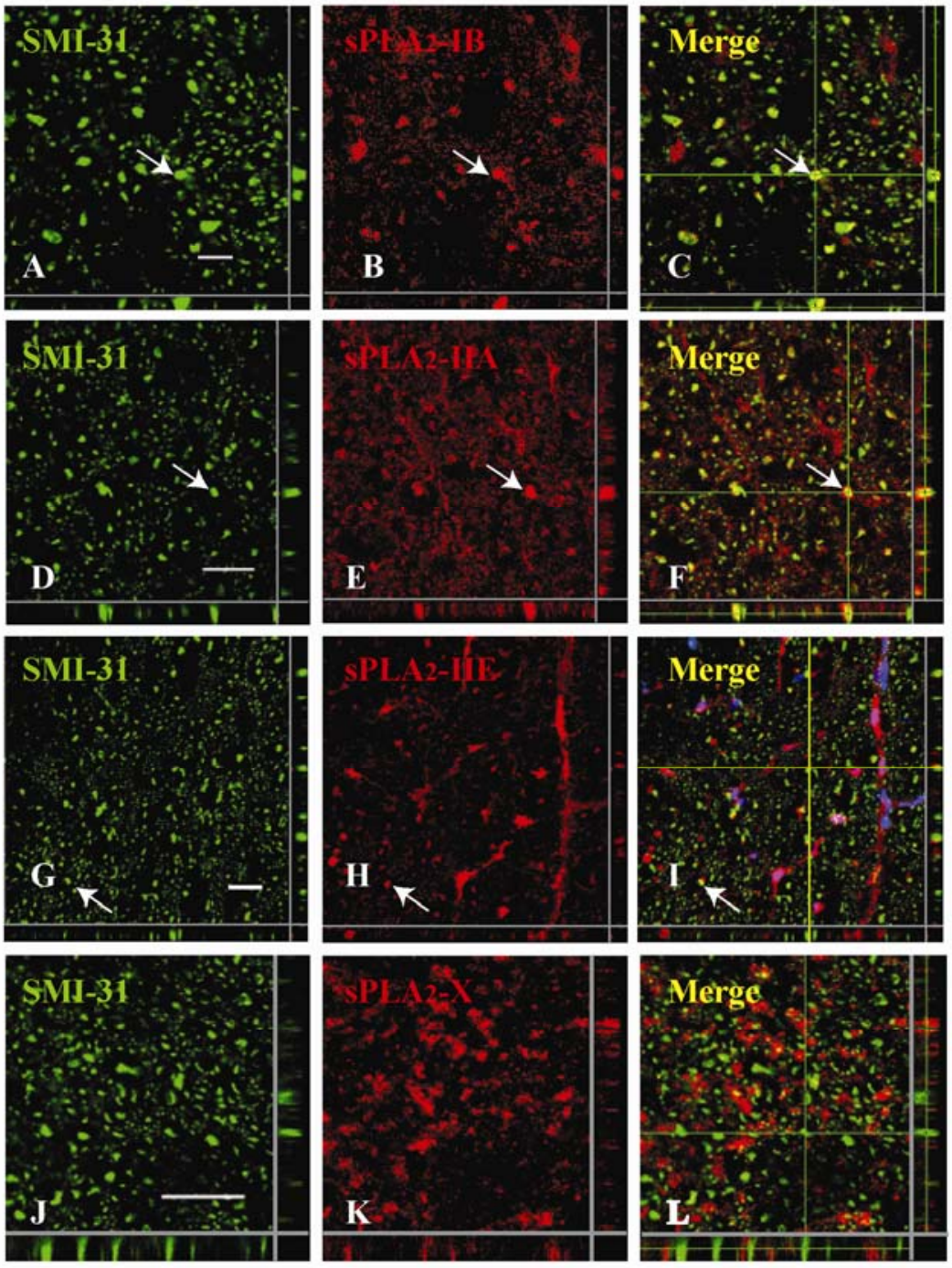


\section{CURRICULUM VITAE}

NAME: $\quad$ William Lee Titsworth

ADDRESS: Kentucky Spinal Cord Injury Research Center

Department of Anatomical Sciences and Neurobiology

611 MDR

511 S. Floyd St.

Louisville, KY 40202

Telephone: $\quad 502-797-4941$

Fax: $\quad$ 502-852-5148

Email: $\quad$ lee.titsworth@louisville.edu

\section{RESEARCH INTEREST:}

My current investigations focus on phospholipases, particularly $\operatorname{sPLA}_{2}$, and their expression following spinal cord injury, what role they might have on demyelination, and what behavioral and histological effects might be seen from inhibition of its activity. My career goal is to function as both a practicing neurologist in an academic hospital as well as maintain a basic science research lab that will allow me to take ideas from "bench to bedside."

EDUCATION:

Present M.D. Current Class Rank 10 out of 146

University of Louisville (Louisville, KY)

Present Ph.D. Anatomical Science and Neurobiology

University of Louisville (Louisville, KY)

$2007 \quad$ M.S. Anatomical Science and Neurobiology

University of Louisville (Louisville, KY)

$2001 \quad$ B.A. Psychology - Summa Cum Laude

Samford University (Birmingham, $A L$ )

BOARD SCORES:

$6 / 06$

USMLE Step $1=250$

$(99 \%)$

$8 / 01$

MCAT Physical Science $=11$

$(79-87 \%)$

Biological Science $=11 \quad(79-87 \%)$ 


$$
\begin{aligned}
& \text { Verbal Reasoning }=9 \\
& \text { Writing Sample }=\mathrm{S}
\end{aligned}
$$

AWARDS and HONORS:

National MD/PhD Student Conference - Oral Presentation / Travel Grant - 2008

Kentucky Spinal Cord and Head Injury Symposim - Travel Grant - 2008

Hays Threlkeld Memorial Scholarship - Univeristy of Louisville - 2003-2010

American Academy of Neurology - Travel Grant - 2005

Externship in Robotic Surgery (Padua, Italy) - 2004

Summer Scholar Award - University of Louisville - 2003, 2004

American College of Surgeons - Travel Grant - 2003

Summa Cum Laude - Samford University - 2001

Janice Teal Award - Samford University, Department of Psychology - 2001

Honors Seminar Scholarship for Study Abroad - Samford University - 2000

Leadership Scholarship - Samford University - 1997-2001

Research Samford - Winner; 2001, 2000

COMMITTEES:

2007 - Search Committee for the Vice Dean for Research

2007 - Faculty Form

TEACHING:

2007/08 - Junior Faculty, Medical Neuroanatomy

2006/07 - Teaching Assistant, Medical Neuroanatomy

PUBLICATIONS and PRESENTATIONS:

Titsworth WL, Cheng X, Ke Y, Burckardt KA, Pendleton C, Liu NK, Shao H, Cao QL, and Xu XM, (2008) Differential Expression of sPLA2 Following Spinal Cord Injury and a Functional Role for sPLA2-IIA in Mediating Oligodendrocyte Death. (In Preparation)

Titsworth WL, Liu NK, Xu XM (2008) Role of Secretory Phospholipase $\mathrm{A}_{2}$ in CNS Inflammation: Implications in Traumatic Spinal Cord Injury. CNS \& Neurological Disorders - Drug Targets. 7:254-269.

Titsworth WL, Onifer SM, Liu NK, Xu XM (2007) Focal phospholipases A 2 group III injections induce cervical white matter injury and functional deficits with delayed recovery concomitant with Schwann cell remyelination. Exp Neurol 207:150-162.

Liu NK, W.L. Titsworth, X.M. Xu,. (2007). Phospholipase $\mathrm{A}_{2}$ in CNS disorders: Implication on traumatic spinal cord and brain injuries. In: N Banik and SK Ray (Editors) Handbook of Neurochemistry and Molecular Neurobiology, 3rd Ed, Springer, New York (In press)

LiU, N. K., Zhang, Y. P., Titsworth, W. L., Jiang, X., Han, S., Lu, P. H., Shields, C. B. \& XU, X. M. (2006). A Novel Role of Phospholipase $A_{2}$ in Mediating Spinal Cord Secondary Injury. - Anal Neurology 59, 606-619. (Anal of Neurology ranks 9 among 200 neuroscience journals).

(Oral Presentations) 
Titsworth WL (2008) $\underline{S P L A}_{2}$ inhibition preserves oligodendrocytes following cytotoxic injury in vitro and creates histological and functional sparing in severe spinal cord injury. National MD/PhD Student Conference, Keystone CO.

(Posters)

Titsworth WL, Zhang YP, Liu NK, Cheng X, Zheng Y, Cao QL, Shields CB, and Xu $\mathrm{XM},(2008) \underline{\mathrm{SPLA}}_{2}$ inhibition preserves oligodendrocytes following cytotoxic injury in vitro and creates histological and functional sparing in severe spinal cord injury. Ketucky Spinal Cord and Head Injury Trust Symposium, Lexington, KY.

Burckardt K, Titsworth WL, Xu XM, 2007. Inhibition of Secretory Phospholipase $\mathrm{A}_{2-}$ IIA Induced Oligodendrocyte Death Following Injury. Proceedings of the Research! Louisville, Louisville, KY.

Titsworth WL, Onifer SM, Liu NK, Xu XM, 2007. Secretory phospholipases $\mathrm{A}_{2}$ induce cervical white matter injury and functional recovery concomitant with Schwann cell remyelination. Proceedings of the Neuroscience Day, Louisville, KY.

Liu, N., Y. P. Zhang, W.L. Titsworth, A.I. Xhafa, C. B. Sheilds, \& X. M. Xu. (2006). Characteristics of phospholipase A2-induced spinal cord injury, a comparison with contusive spinal cord injury in adult rats. Society for Neuroscience, Annual Meeting, Atlanta, GA.

Rukavina, M., J. Pei, W. L. Titsworth, Y. Zou and X. M. Xu (2006). Effects of Inhibition of Wnt on axonal regeneration of the damaged corticospinal tract following spinal cord dorsal hemisection. Research Louisville, Louisville, KY.

Cox, B., J. Pei, W. L. Titsworth, N. K. Liu and X. M. Xu (2004). The effect of phospholipase A2 on schwann cell proliferation. Research Louisville, Louisville, KY.

FUNDING:

Role of sPLA2 in Post Spinal Cord Injury Demyelination

$\mathrm{NIH}$ - Individual Predoctoral Fellowship (F-31)

1F31NS056574-01A1

05/06-10/06 \$101,985.15

CONFERENCES ATTENDED:

2008 - Medical Scientist Training Program Annual MD/PhD Student Conference

2008 - '03 - Kentucky Head and Spinal Cord Injury Symposium

2007 - NINDS/AUPN/ANA "How to combine clinical and research careers in

neuroscience." symposium - refereed attendance

2006 - Society for Neuroscience - Poster Presentation

2006 - Neurology and Neurosurgery Pediatric Review

2003 - Congress of the American College of Surgeons

2001 - National Conference on Undergraduate Research (NCUR), Presenter

2001 - Southeast Regional Psychology Confernce, Presenter

2000 \& 2001 - Research Samford

PROFESSIONAL SOCIETIES:

Society for Neuroscience 
American Medical Association

American Academy of Neurology

Scoiety for Vascular and Interventional Neurology

American Medical Student Association 